\title{
Numerical and Experimental Analyses of Aerosol Deposition in a Novel and Standardized Human Nasal Cavity
}

by

\section{Yuan Liu, M.A.Sc. Mechanical Engineering}

\author{
A Thesis submitted to \\ the Faculty of Graduate Studies and Research \\ in partial fulfilment of \\ the requirements for the degree of \\ Doctor of Philosophy \\ in Mechanical Engineering \\ Ottawa-Carleton Institute for \\ Mechanical and Aerospace Engineering \\ Department of Mechanical and Aerospace Engineering \\ Carleton University \\ Ottawa, Ontario, Canada \\ March 31, 2010
}

Copyright (C)

2010 -Yuan Liu 
Library and Archives

Canada

Published Heritage

Branch

395 Wellington Street

Ottawa ON K1A ON4

Canada
Bibliotheque et

Archives Canada

Direction du

Patrimoine de l'édition

395, rue Wellington

Ottawa ON K1A ON4

Canada
Your file Votro référence

ISBN: 978-0-494-67866-4

Our file Notre reference

ISBN: 978-0-494-67866-4
NOTICE:

The author has granted a nonexclusive license allowing Library and Archives Canada to reproduce, publish, archive, preserve, conserve, communicate to the public by telecommunication or on the Internet, loan, distribute and sell theses worldwide, for commercial or noncommercial purposes, in microform, paper, electronic and/or any other formats.

The author retains copyright ownership and moral rights in this thesis. Neither the thesis nor substantial extracts from it may be printed or otherwise reproduced without the author's permission.
AVIS:

L'auteur a accordé une licence non exclusive permettant à la Bibliothèque et Archives Canada de reproduire, publier, archiver, sauvegarder, conserver, transmettre au public par télécommunication ou par l'Internet, prêter, distribuer et vendre des thèses partout dans le monde, à des fins commerciales ou autres, sur support microforme, papier, électronique et/ou autres formats.

L'auteur conserve la propriété du droit d'auteur et des droits moraux qui protège cette thèse. $\mathrm{Ni}$ la thèse ni des extraits substantiels de celle-ci ne doivent être imprimés ou autrement reproduits sans son autorisation.
In compliance with the Canadian Privacy Act some supporting forms may have been removed from this thesis.

While these forms may be included in the document page count, their removal does not represent any loss of content from the thesis.
Conformément à la loi canadienne sur la protection de la vie privée, quelques formulaires secondaires ont été enlevés de cette thèse.

Bien que ces formulaires aient inclus dans la pagination, il n'y aura aucun contenu manquant.

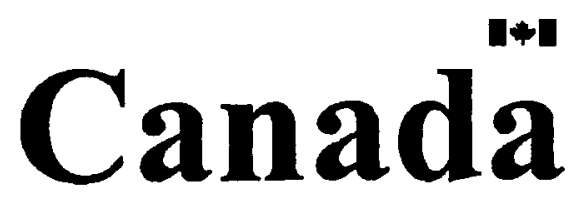




\section{Abstract}

This thesis presents an investigation of flow and aerosol deposition patterns in a model human nasal cavity using both experimental measurements and numerical simulations. A novel, standardized human nasal cavity model was created as part of this work by processing 30 sets of computed tomography (CT) scans of nasal airways of healthy subjects. Aerosol deposition in the novel, "Carleton-Civic" standardized geometry of the human nasal cavity was studied both numerically and experimentally. Inhalation flow rates varied from 30 to $90 \mathrm{~L} / \mathrm{min}$ in the experiments, and aerosol droplets had diameters ranging from 1.71 to $9.14 \mu \mathrm{m}$, giving impaction parameters ranging from 123.3 to $2527.6 \mu \mathrm{m} \mathrm{L} / \mathrm{min}$. For the numerical simulations, both the RANS/EIM (Reynolds Averaged Navier-Stokes equations for the gas phase and Eddy-Interaction random walk Models for the particulate phase) and Large Eddy Simulations were used to better understand the limits of applicability and accuracy of standard numerical methods as available in many commercial packages.

Both experimental and simulated results showed that the mechanisms of aerosol deposition in the standardized nasal cavity were dominated by inertial impaction. Measured deposition data from the standardized nasal cavity transected cited in vitro data based on individual subjects. The data correlated very well with cited in vivo measurements but generally showed less aerosol deposition for a given value of the impaction parameter. Regional particle deposition characteristics within the nasal passages were also investigated both experimentally and numerically, and new trends of 
regional deposition versus impaction parameter are discussed. These provide new insight into the general deposition behaviour of various sized aerosols within the human nasal cavity. 


\section{Acknowledgements}

There were many people who were helpful and supportive while I was pursuing my $\mathrm{PhD}$. I want to take this opportunity to express my gratitude to these people who offered me their help in the years that I was performing my research on aerosols.

First, I want to extend many thanks to my thesis supervisors, Dr. Matthew Johnson and Dr. Edgar Matida, who offered me their guidance, patience, encouragement and support not only in my research, but also in my personal life, especially during the time when I was expecting my baby girl. Working with them has been an invaluable experience and will be priceless in my career and personal life.

I am also grateful to my friends within our research group for their help while I was preparing my experimental setup. I want to acknowledge, in particular, the contributions of Brian and Scott.

Thanks also go to Steve, Stefan, Gary, Alex, Kevin and Fred for their help in generating the nasal cavity model and setting up my experiments. I am also very grateful to Nancy, Christie and Marlene for their kind helps throughout my $\mathrm{PhD}$ degree program.

I would like to thank my husband, Ying Chen, my parents, my parents-in-law, and my sister for their complete understanding and unconditional support. I send my love to my little girl, Sophia. I am so thankful for the confidence and courage she gives me every day. 


\section{Table of Contents}

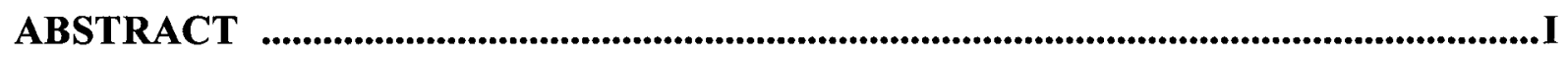

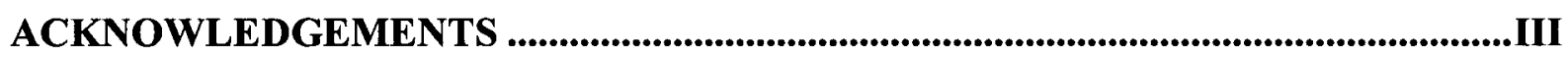

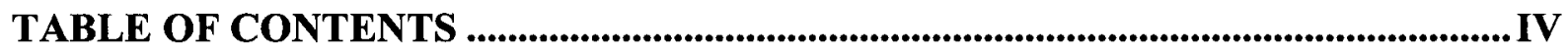

LIST OF TABLES ..........................................................................................................

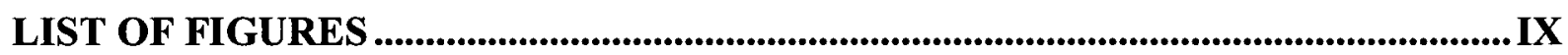

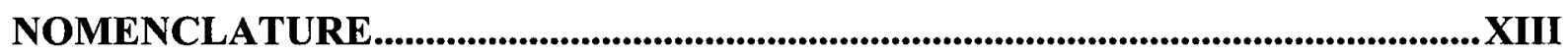

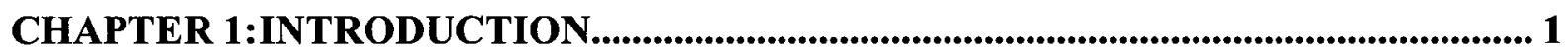

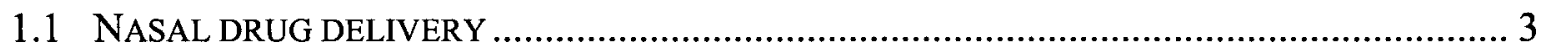

1.2 NASAL ANATOMY AND RESPIRATORY PHYSIOLOGY .................................................. 4

1.3 TWO-PHASE FLOW AND MECHANISMS OF PARTICLE DEPOSITION ..................................... 8

1.3.1 Particle Deposition...............................................................................................

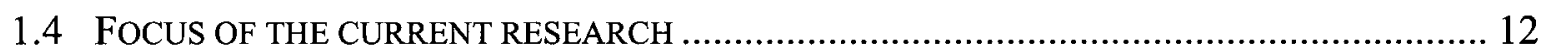

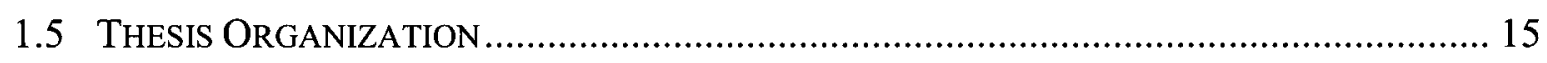

CHAPTER 2:LITERATURE REVIEW .................................................................. 16

2.1 PREVIOUS MEASUREMENTS OF CHARACTERISTIC DIMENSIONS IN HUMAN NASAL

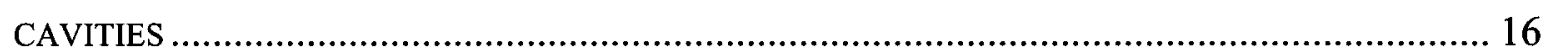

2.1.1 Measurements Using Acoustic rhinometry ..................................................................18

2.1.2 Nasal Cavity Measurements using Computed Tomography and Magnetic Resonance Imaging 19

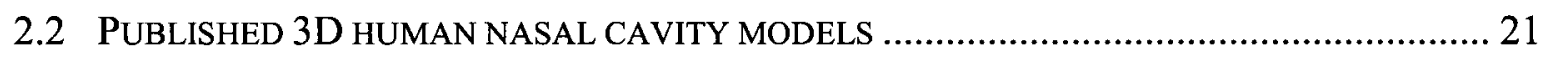

2.2.1 Methods of Manufacturing Nasal Cavity Replicas..............................................................25

2.3 EXPERIMENTAL STUDIES OF AIRFLOW PATTERNS AND PARTICLE DEPOSITION IN

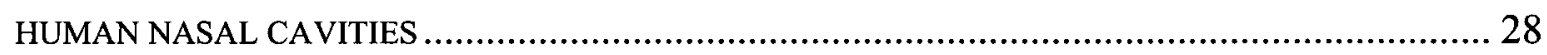

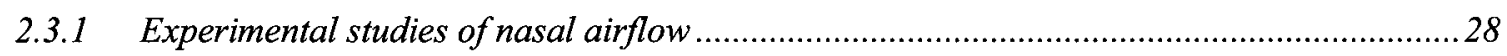

2.3.2 Experimental study of particle deposition in the airway passages........................................33

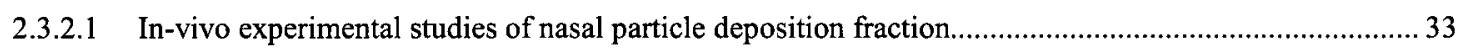

2.3.2.2 In vitro experimental studies on nasal particle deposition fraction...................................................... 37

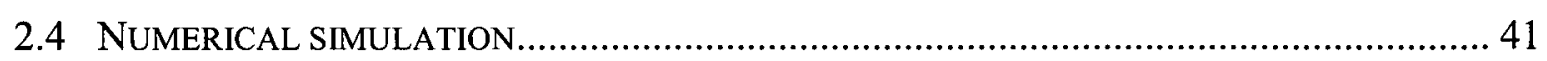

2.4.1 Overview of simulation of particles in turbulent flow ...................................................

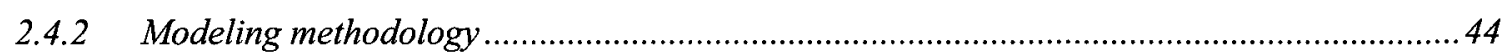


2.4.3 Previous Simulations of particles in human respiratory tract .......................................... 47

2.5 Geometry Related Limitations of Previous Work ........................................ 50

CHAPTER 3:CONSTRUCTION OF REALISTIC MODELS OF THE HUMAN

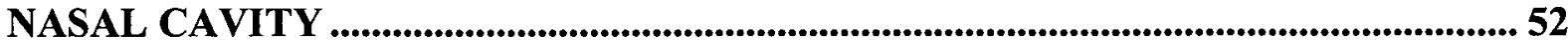

3.1 Baseline Data Used to CREate a Standardized Human Nasal CaVity

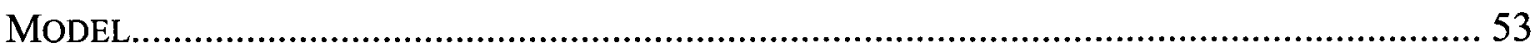

3.2 GENERATION AND ALIGNMENT OF INDIVIDUAL 3D NASAL CAVITY MODELS ...............55

3.2.1 Construction of coarse $3 \mathrm{D}$ models from $\mathrm{CT}$ scans .............................................................55

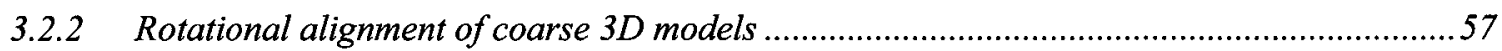

3.2.2.1 Specifying the landmark and choana from the raw CT scans ................................................................5 58

3.2.2.2 Rotating coarse nasal models ..................................................................................................... 59

3.3 CREATION OF THE STANDARDIZED HUMAN NASAL CAVITY MODEL ........................... 62

3.3.1 Measuring characteristic length and scaling individual coarse models .............................62

3.3.2 Slicing and smoothing individual coarse models .........................................................64

3.3.3 Measurement of characteristic dimensions of individual coarse 3D nasal cavity models .... 68

3.3.4 Image processing methodology for generating median coronal plane images ................... 70

3.3.5 Determine the shape of nostril and outlet of the standardized nasal cavity ......................... 74

3.3.6 Construction of the final Carleton-Civic standardized nasal model .................................... 75

3.4 ANALYSIS OF THE CARLETON-CIVIC STANDARDIZED NASAL MODEL ......................... 76

CHAPTER 4:METHODOLOGY OF EXPERIMENTAL MEASUREMENT OF PARTICLE DEPOSITION IN HUMAN NASAL CAVITY ........................................... 82

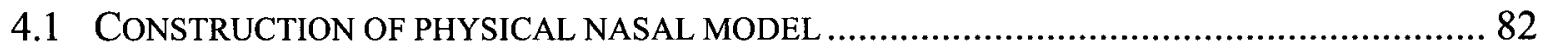

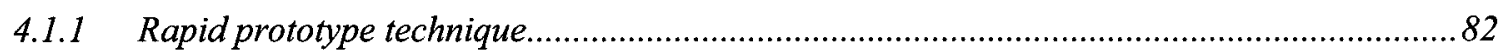

4.1.2 Creation of physical nasal model using $R P$ machine ...................................................... 83

4.2 EXPERIMENTAL APPARATUS AND PROCEDURES .................................................. 86

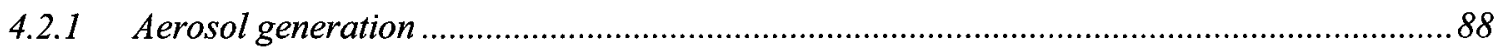

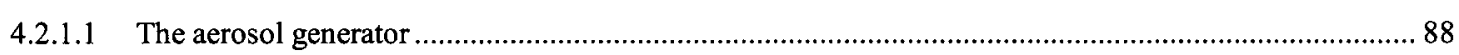

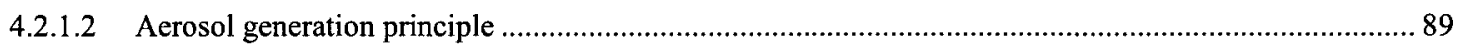

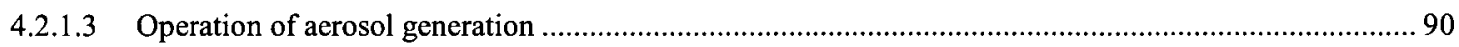

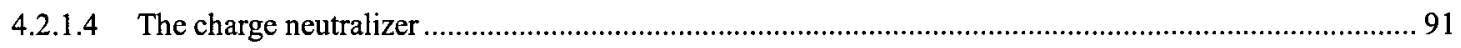

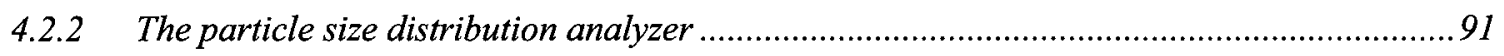

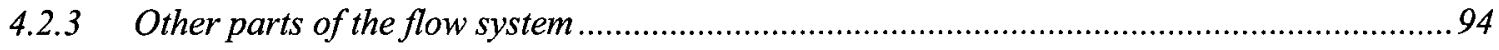

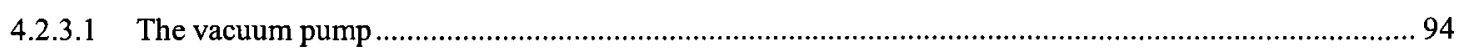

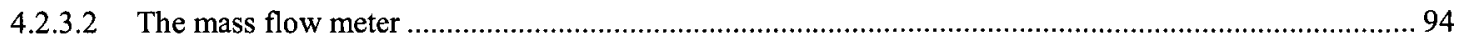

4.2.4 Particle deposition measurement …..................................................................... 95 


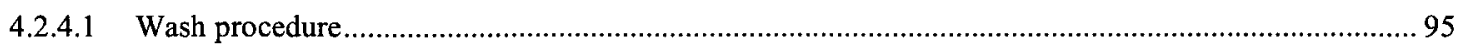

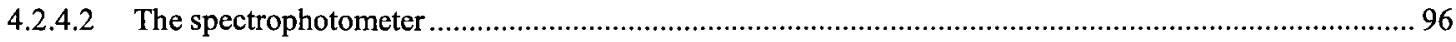

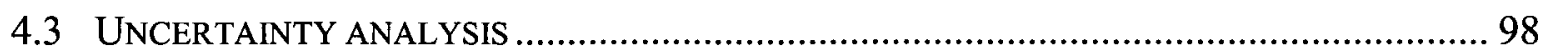

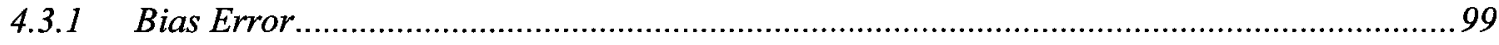

4.3.1.1 Inertial parameter ............................................................................................................. 100

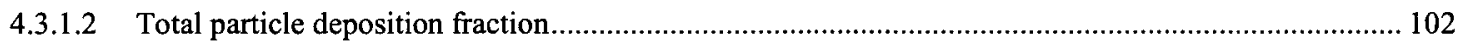

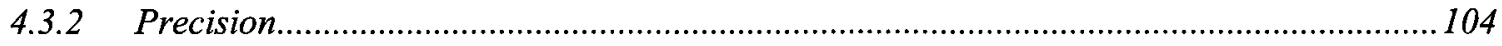

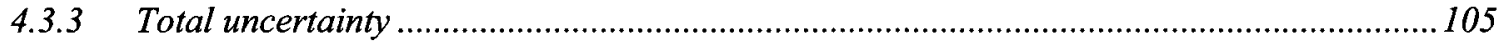

CHAPTER 5:EXPERIMENTAL DEPOSITION RESULTS ...................................... 107

5.1 MEASURED AND SIMULATED TOTAL PARTICLE DEPOSITION FRACTION ..................... 107

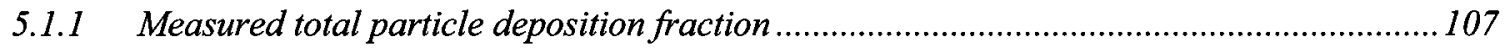

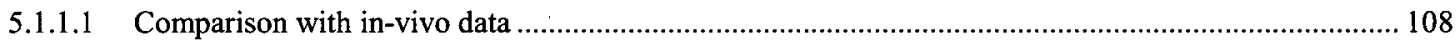

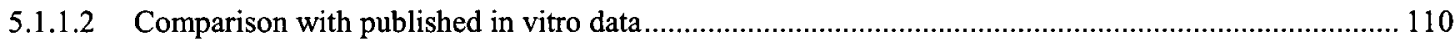

5.1.2 Measured regional particle deposition fraction ..............................................................113

CHAPTER 6:NUMERICAL SIMULATION METHODOLOGY .................................. 115

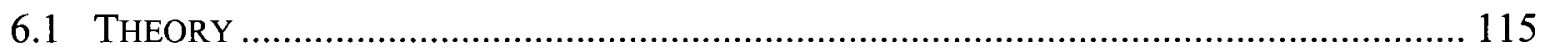

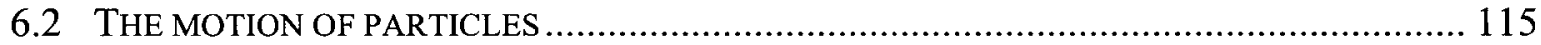

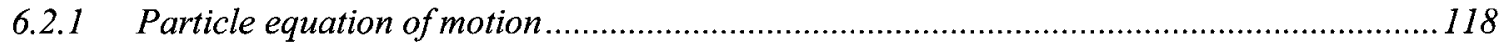

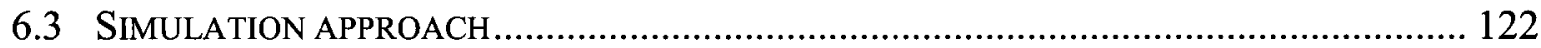

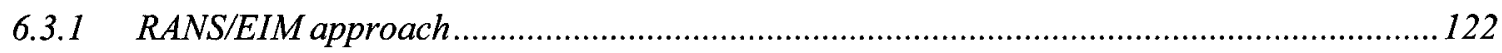

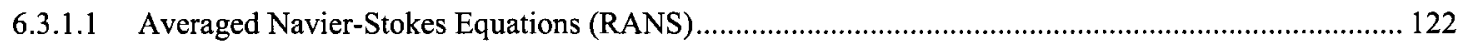

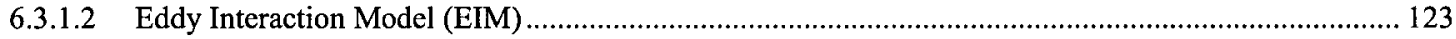

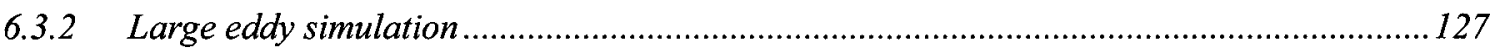

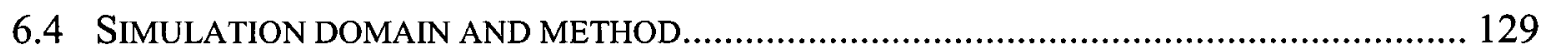

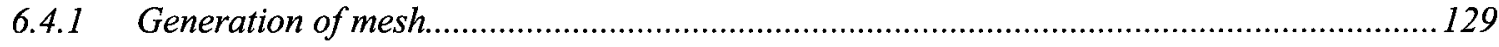

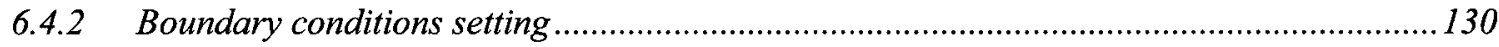

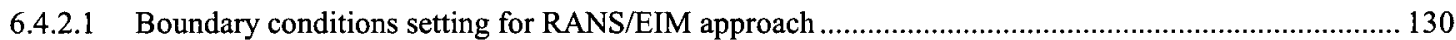

6.4.2.2 Boundary conditions and solve setting for LES ............................................................................. 134

CHAPTER 7:SIMULATION RESULTS ........................................................................ 135

7.1 GRID CONVERGENCE ANALYSIS .......................................................................... 135

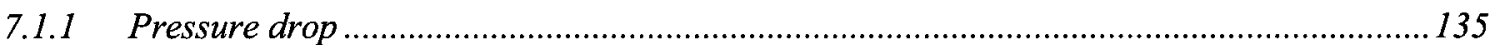

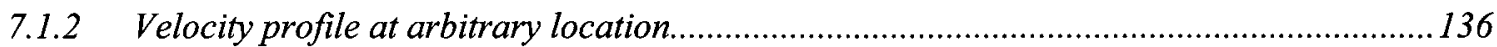

7.1.3 Grid effect on particle deposition fraction ..............................................................................137

7.1.4 Effect of iteration numbers on LES simulation results ..................................................138

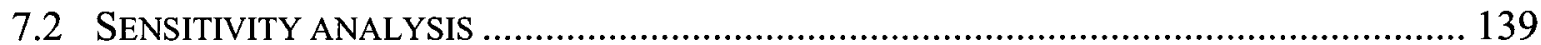


7.3 AIRFLOW STRUCTURE

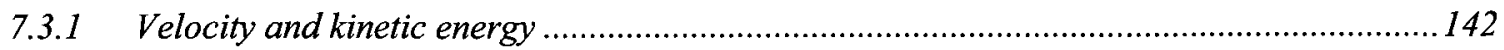

7.3.1.1 Distribution of velocity and turbulence kinetic energy ..................................................................... 142

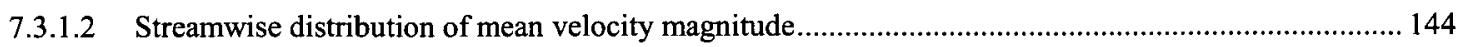

7.3.1.3 Streamwise distribution of turbulence kinetic energy ......................................................................... 145

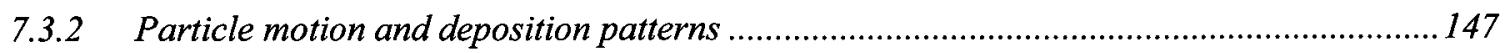

7.3.3 Simulated results of total particle deposition fraction ...................................................... 148

7.3.3.1 Effect of different RANS model on particle deposition fraction ......................................................... 148

7.3.3.2 Simulated results of total particle deposition fraction........................................................................ 149

7.3.4 Regional particle deposition fraction results ............................................................... 153

7.3.4.1 Simulated results of regional particle deposition fraction distribution ................................................ 153

7.3.4.2 Comparison of measured and computed regional particle deposition fraction ..................................... 156

7.3.4.3 Fitting curves for regional particle deposition fraction....................................................................... 157

7.3.5 Effect of size of nasal cavity on particle deposition fraction ............................................158

CHAPTER 8:CONCLUSIONS AND FUTURE WORK .................................................... 160

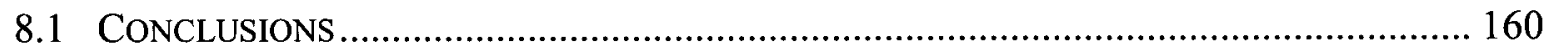

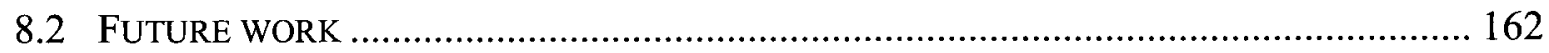

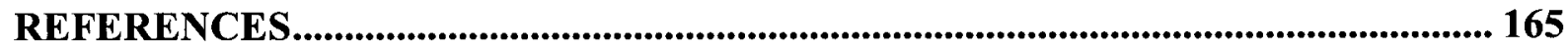

APPENDIX A: SHEAR STRESS TRANSPORT (SST) TURBULENCE MODEL..... 177

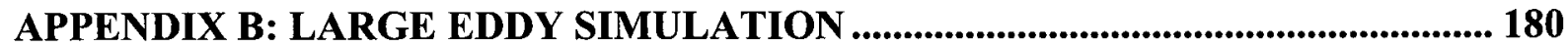

GLOSSARY 


\section{List of Tables}

Table 1.1 Fluid dynamic description of human respiration (as summarized by Tang et al., 2004). 8

Table 1.2 Two-phase flow in various engineering systems, summarized by Loth (2009) . 9

Table 2.1 Summarized measurements of characteristic dimensions of human nasal cavities

Table 2.2 Summary of 3D human nasal cavity models 23

Table 2.3 Summary of previous numerical simulations of particle deposition in human nasal cavity. 48

Table 3.1 Information about the subjects and CT scan of 30 individuals in current study

Table 3.2 Measurements of geometric parameters of the middle region of aligned scaled coarse nasal models.

Table 3.3 Measurements of frontal/nostril regions from 7 sets of CT scans 69

Table 3.4 Averaged dimensions of the nostril inlet plane (merged raw data from Table 3.3 and from Kesavanathan and Swift (1998)) compared with final values for the Carleton-Civic Standardized Model 74

Table 4.1 Bias limits of the key parameters of total particle fraction measurement ...... 104

Table 5.1 Experimental Results and Uncertainty ......................................................... 108

Table 6.1 Force exerted on immersed objects ................................................................ 115

Table 6.2 Velocities and Reynolds numbers for different flow rates ........................... 132

Table 6.3 Physical particle diameters, $d_{p}(\mu m)$, used in simulations for different values of the impaction parameter and flow rate.

Table 7.1 Calculation parameters for sensitivity analysis (constant inlet length scale) . 140

Table 7.2 Calculation parameters for sensitivity analysis (constant inlet length scale) 141

Table 7.3 The values of turbulence kinetic energy at the small peak for low, middle and

high turbulence intensities at the inlet... 147

Table 7.4 Curve fit parameters for regional deposition data 158 


\section{List of Figures}

Figure 1.1 Anatomical illustration of the human nasal cavity of a sagittal plane cut (a) and sample coronal plane midway through the nasal cavity (b)

Figure 2.1 Cross-sectional areas of nasal models (green curve) derived from published data of CT scans and MRI scans.

Figure 2.2 Side view of physical nasal cavity model created by Schreck et al., (1993)... 26

Figure 2.3 Deposition fraction in human nasal cavity illustrated as a function of impaction parameter for an adult human volunteer (Pattle, 1961) 34

Figure 2.4 Summary of nasal deposition fraction as a function of impaction parameter in human volunteers (after Cheng, 2003).

Figure 2.5 Particle deposition in the nasal cavity using an in vivo scintigraphy method (left: using passive nebulizer; right: using nasal spray pump (Giroux, 2005) 37

Figure 2.6 Summary of in vitro measurements of nasal deposition fraction .................... 39

Figure 2.7 Interactions between particles and fluid turbulence ...................................... 42

Figure 2.8 Previous simulation results of particle deposition fraction ............................. 50

Figure 3.1 Procedure of generation of coarse nasal cavity model using 3D-Doctor software. 56

Figure 3.2 Identification of the (a) maxillary spine landmark and (b) choana in the raw, coronal plane CT scans . 58

Figure 3.3 Coordinate system for rotating the coarse 3D nasal cavity model. Example images show coarse model 8

Figure 3.4 Illustration of the first two steps of rotational alignment of a coarse 3D nasal cavity model. (Example images show coarse model 8.) (a) Top view of model before rotation; (b)Top view of model after rotation around imaginary reference line linking the maxillary spine landmark and the posterior merge point; (c)Top view of model after rotation around $\mathrm{Y}$-axis to align symmetry plane with $\mathrm{X}$-axis.

Figure 3.5 Example images of model number 8 showing rotational alignment of a coarse $3 \mathrm{D}$ nasal cavity model. Original orientation is shown in green and final, aligned 
model is shown in red. The positions of the imaginary reference lines before and after alignment are indicated.

Figure 3.6 Three sub-regions of a 3D nasal cavity

Figure 3.7 Number of available coronal-plane images at each X-location along nasal cavity. 65

Figure 3.8 Example of cross-sectional boundary smoothing. The dashed grey curve is the original boundary, points are manually selected on this boundary, and the black continuous curve is the corrected and smoothed boundary.... 66

Figure 3.932 out of 60 nasal cavity cross-sectional shapes located $30 \mathrm{~mm}$ from the maxillary spine landmark 68

Figure 3.10 Example coronal plane images at $X=30 \mathrm{~mm}$ obtained during processing to determine the characteristic cross-sectional geometry. a) ensemble averaged grayscale image; b) binarized 50\% threshold ("median") image; c) median image in red overlaid on top of "median area" image in yellow; d) final boundaries of cavity at current $\mathrm{X}$-position in yellow, at next upstream position in green, at next downstream position in red, overlaid on median image in blue 71

Figure 3.1156 cross-sectional shapes for the standardized human nasal cavity ............. 73

Figure 3.12 Final standardized nasal cavity model. a) Top view, b) Side view; c) Bottom view

Figure 3.13 Cross-sectional area of the Carleton-Civic standardized nasal model (green curve) compared with individual (dots), median (red curve), and average (blue curve) cross-sectional areas from all available subjects

Figure 3.14 Cross-sectional areas of the standardized nasal model (green curve) and 14 complete nasal passages (black curve). Running average of the 14 complete curves is shown for comparison in blue

Figure 3.15 Comparison of cross-sectional areas of the Carleton-Civic standardized nasal model (green curve) with published CT scan derived data from Min and Jang (1995) (orange and red curves), Terheyden et al. (2000) (crosses / blue curve) and Kelly et al. (2000) (circles); and MRI derived data from Cheng Y.S. et al. (1996) (inverted triangles / purple curve) and Corey et al. (1997) (yellow curve). 
Figure 4.1 Carleton-Civic standardized nasal cavity used for deposition experiments in the ICEM-CAD environment with transparent outer surfaces.

Figure 4.2 Carleton-Civic standardized nasal cavity used for deposition experiments:

three regions of the physical model along with the two connection pieces 86

Figure 4.3 Schematic of experimental apparatus.

Figure 4.4 TIS Model 3450 Vibrating Orifice Aerosol Generator (left, including the TSI Model 3054 Aerosol Neutralizer) and TIS 3603 Particle Size Distribution Analyzer (right) 89

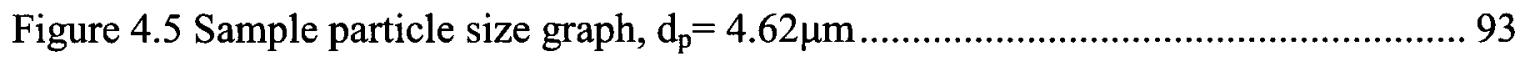

Figure 4.6 Agilent 8453 UV-visible Spectroscopy system............................................. 96

Figure 4.7 Errors in the measurement of a variable X (Coleman \& Steele, 1999) ........... 98

Figure 5.1 Experimental results of total particle deposition fraction compared with published in vivo data

Figure 5.2 Experimental results of total particle deposition fraction compared with published in vitro data

Figure 5.3 Experimental measurements of regional particle deposition fraction in the Carleton-Civic standardized nasal cavity geometry.

Figure 6.1 Drag coefficient (Lapple and Shepard, 1940) .....

Figure 6.2 Eddy-particle interaction model

Figure 6.3 Sample of terahedual and prismatic elememts for 4000000 elememts

Figure 7.1 Simulation results of the pressure drop through nasal cavity compared with experimental results (Weinhold et al., 2004)

Figure 7.2 Grid size effect on velocity distribution on arbitrary line, which is on the cross section cross section $66 \mathrm{~mm}$ posterior to the nostril (flow rate $=30 \mathrm{~L} / \mathrm{min}$ )

Figure 7.3 Effect of computational grid size on particle deposition fraction $(\mathrm{Q}=60 \mathrm{~L} / \mathrm{min})$

Figure 7.4 LES results of total particle deposition fraction as function of number of iterations

Figure 7.5 Effect of fractional turbulence intensity $I$ on particle deposition fraction $(\mathrm{Q}$ $=60 \mathrm{~L} / \mathrm{min}$, length scale at inlet $=D_{h} / 10$ ) 
Figure 7.6 Effect of inlet length scale ratio on particle deposition fraction $(\mathrm{Q}=60 \mathrm{~L} / \mathrm{min}$, fractional turbulence intensity $I=0.1$ )

Figure 7.7 Velocity distribution (a) and turbulence kinetic energy distribution (b) in standardized human nasal cavity along coronal planes (flow rate, $Q=60 \mathrm{~L} / \mathrm{min}, 4$ 000000 elements)

Figure 7.8 Streamwise distribution of mean velocity magnitude

Figure 7.9 Average cross-stream turbulence kinetic energy for low (1\%), middle (5\%) and high (10\%) turbulence intensities at inlet.

Figure 7.10 Particle tracking with particle travel distance $(\mathrm{Q}=60 \mathrm{~L} / \mathrm{min}, \mathrm{dp}=7.071 \mu \mathrm{m}$, $\mathrm{d}_{\mathrm{p}}^{2} \mathrm{Q}=2736$ ). (a) RANS/EIM turbulence tracking. (b) RANS/EIM mean flow tracking. (c) LES.

Figure 7.11 Particle deposition fraction results using different RANS model (turbulent tracking method) .

Figure 7.12 Deposition results using different simulation methods compared with measured data.

Figure 7.13 Comparison of current simulation results with published simulation data from other researchers

Figure 7.14 Regional particle deposition fraction for different sized particles calculated from LES simulations for inhalation flow rates of: (a) $30 \mathrm{~L} / \mathrm{min}$, (b) $60 \mathrm{~L} / \mathrm{min}$, and (c) $90 \mathrm{~L} / \mathrm{min}$

Figure 7.15 Measured and LES results for regional deposition for various sizes of particles at inhalation flow rates of: (a) $30 \mathrm{~L} / \mathrm{min}$, (b) $60 \mathrm{~L} / \mathrm{min}$, and (c) $90 \mathrm{~L} / \mathrm{min} 156$

Figure 7.16 Particle deposition in the three regions of the nasal cavity correlated with the impaction parameter, $d_{a}^{2} Q$ 157

Figure 7.17 Deposition results using shrunk nasal cavity models 159 


\section{Nomenclature}

\begin{tabular}{|c|c|}
\hline$A$ & Cross-sectional area in the nasal airway $\left[\mathrm{m}^{2}\right]$ \\
\hline$A_{\min }$ & Minimal cross-sectional area in the nasal airway $\left[\mathrm{m}^{2}\right]$ \\
\hline$a^{\prime}$ & Constant in fitting equation of total particle deposition fraction \\
\hline$A b$ & Absorption fraction of a sample measured by the spectrophotometer [-] \\
\hline$B$ & Bias limits or body force $[\mathrm{N}]$ \\
\hline$C_{D}$ & Drag coefficient $[-]$ \\
\hline$C$ & Volumetric concentration of Vitamin $\mathrm{E}$ in the vitamin $\mathrm{E}$ solution $\left[\mathrm{g} / \mathrm{cm}^{3}\right]$ \\
\hline$C_{a}$ & Added mass coefficient $[-]$ \\
\hline$C_{\mu}$ & A constant in the eddy diffusivity formula [-] \\
\hline$D F$ & Deposition fraction [-] \\
\hline$D$ & Diffusion coefficient [-] \\
\hline$d$ or $d_{p}$ & Particle diameter $[\mu \mathrm{m}]$ \\
\hline$d_{a}$ & Aerodynamic diameter of a particle $\left(\rho_{p} / \rho_{w}\right)^{1 / 2} d_{p}[\mu \mathrm{m}]$ \\
\hline$\frac{D}{d t}$ & Total time derivative for a fluid particle $[-]$ \\
\hline$\frac{d}{d t}$ & Total time derivative for an aerosol particle (or for a fluid particle) $[-]$ \\
\hline $\bar{F}_{\text {Drag }}$ & Drag force on aerosol particle due to the air $[\mathrm{N}]$ \\
\hline$F_{\text {Saffmann }}$ & Saffman lift force $[\mathrm{N}]$ \\
\hline$f$ & Frequency of the aerosol generator (also used as an arbitrary function) \\
\hline$F$ & $\begin{array}{l}\text { A function in the turbulence model (or in Appendix A, inertial body force } \\
{[\mathrm{N}] \text { ) }}\end{array}$ \\
\hline$g$ & Gravitational acceleration constant $\left[\mathrm{m} / \mathrm{s}^{2}\right]$ \\
\hline$I$ & $\begin{array}{l}\text { Volumetric concentration of non-volatile impurities }\left[\mathrm{g} / \mathrm{cm}^{3}\right] \text {; also used to } \\
\text { denote turbulence intensity[-] }\end{array}$ \\
\hline$l_{e}$ or $L_{e}$ & Length scale of the energy containing eddies [m] \\
\hline$l_{t}$ & Specified length scale at inlet [m] \\
\hline
\end{tabular}




\begin{tabular}{|c|c|}
\hline In & Incident light on a sample in the spectrophotometer [-] \\
\hline$k$ & Boltzmann's constant (or turbulence kinetic energy) [-] \\
\hline$m$ & $\operatorname{mass}[\mathrm{kg}]$ \\
\hline$\sqrt{2}$ & Mass flow rate of air $[\mathrm{kg} / \mathrm{s}]$ \\
\hline$M$ & Number of particles or number of readings in measurement $[-]$ \\
\hline$M_{n}$ & Masses of particles deposited in the nasal model $[\mathrm{kg}]$ \\
\hline$M_{f}$ & Masses of particles deposited in the filter [kg] \\
\hline$N$ & Vol times $\mathrm{Ab}[-] ;$ also used to denote a random number[-] \\
\hline$P$ & Air pressure $[\mathrm{Pa}]$ \\
\hline$P_{\text {gauge }}$ & Gauge pressure of air $[\mathrm{Pa}]$ \\
\hline$P_{r}$ & Precision limit of the measured results $[-]$ \\
\hline$Q$ & Volume flow rate of air $\left[\mathrm{m}^{3} / \mathrm{s}\right]$ \\
\hline$Q_{S}$ & Volume flow rate of vitamin $\mathrm{E}$ solution $\left[\mathrm{m}^{3} / \mathrm{s}\right]$ \\
\hline$Q_{S L P M}$ & Flow rate of air assuming atmospheric air density $\left[\mathrm{m}^{3} / \mathrm{s}\right]$ \\
\hline$r$ & Readings in experiment $[-]$ \\
\hline $\bar{r}$ & Averaged result [-] \\
\hline $\operatorname{Re}$ & $\begin{array}{l}\text { Flow Reynolds number based on the pipe diameter and the area-average } \\
\text { velocity through the pipe [-] }\end{array}$ \\
\hline$R e_{p}$ & Reynolds number of aerosol particle $[-]$ \\
\hline$R e_{\lambda}$ & Turbulent Reynolds number [-] \\
\hline$S G$ & Specific Gravity [-] \\
\hline$S_{\bar{r}}$ & Standard deviation $[-]$ \\
\hline Stk & Stokes number [-] \\
\hline$t$ & Time $[\mathrm{s}]$, or coverage factor to calculate precision limit \\
\hline$t_{0}$ & The starting time $[\mathrm{s}]$ \\
\hline$T$ & Temperature $[\mathrm{k}]$ \\
\hline $\operatorname{Tr}$ & Transmitted light for a sample in the spectrophotometer [-] \\
\hline$u$ & Velocity component in the $r$-direction, or the $\mathrm{x}$-direction $[\mathrm{m} / \mathrm{s}]$ \\
\hline$u_{e}$ & Eddy characteristic velocity scale $[\mathrm{m} / \mathrm{s}]$ \\
\hline
\end{tabular}


$U \quad$ Total uncertainty [-]; or instant velocity $[\mathrm{m} / \mathrm{s}]$; also used to denote the relative velocity between the sphere and the fluid $[\mathrm{m} / \mathrm{s}]$

$v$

V

$V_{p}$

Vol

w

$X, Y, Z$

\section{Greek}

$\beta$

$v$ $\delta$

$\rho$

$\rho_{\text {atmosphere }}$

$\mu$

$\mu_{T}$

$\nabla^{2}$

$\nabla$

$\alpha$

$\lambda$

$\tau$

$\tau_{e}$

$\tau_{L}$

$\tau_{p}$

$\tau_{K}$

\section{$\beta$}

(

$\rho$

$\mu$

$\mu$

\section{$\nabla^{2}$}

$\nabla$

$\alpha$

$v$

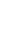

$\tau$

$\tau_{K}$

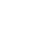

Velocity of air $[\mathrm{m} / \mathrm{s}]$

Velocity component in the $\alpha$-direction, or the y-direction $[\mathrm{m} / \mathrm{s}]$

Area average speed of air. Also used to denote volume occupied by particles and fluid $[\mathrm{m} / \mathrm{s}]$

Volume of a single particle $\left[\mathrm{m}^{3}\right]$

Volume of wash solution $\left[\mathrm{m}^{3}\right]$

Velocity component in the $\theta$-direction, or the $\mathrm{z}$-direction $[\mathrm{m} / \mathrm{s}]$

Rectangular coordinates [-] 
$\tau_{x y} \quad$ Stress on a fluid element, the first subscript referring to the face, the second to the direction $[\mathrm{s}]$

$\Omega \quad$ Absolute value of vorticity [-]

$\Phi_{p} \quad$ Volume fraction of particles [-]

$\Phi, \Phi_{1}, \Phi_{2}$,

$\beta, \beta^{\prime}, \alpha$, Constants in the turbulence model [-]

$\sigma, \sigma^{\prime}$

$\varepsilon \quad$ Dissipation rate of turbulence kinetic energy $\left[\mathrm{m}^{2} / \mathrm{s}^{3}\right]$; also used as precision error [-]

$\omega \quad$ Turbulent frequency

$\Gamma \quad$ Velocity gradient $[1 / \mathrm{s}]$

\section{Subscripts}

$\begin{array}{ll}\mathrm{f} & \text { Fluid or filter } \\ \mathrm{p} & \text { Particle } \\ \mathrm{rel} & \text { Particle relative to the flow field } \\ x, y, z & \text { Eddy } \\ x y, \text { tc } & \mathrm{x}, \mathrm{y}, \mathrm{z} \text { components } \\ 0,1,2 & \text { On the x-component face, pointing in the y-direction, etc. } \\ G & \text { Fluid } 0,1, \text { or } 2 \text { (Appendix A) } \\ B & \text { Relative to the gravity } \\ F & \text { Relative to the body } \\ m & \text { Fluid } \\ m o d e l & \text { Time mean values } \\ n & \text { Model } \\ r m s & \text { Nasal cavity model } \\ t & \text { Root mean square } \\ r e a l & \text { Turbulent } \\ w & \text { Real }\end{array}$




\section{Superscripts}
(0)
Turbulent fluctuations
Undisturbed flow field
(1) Perturbation of the flow field
(2) Perturbed flow field 


\section{Chapter 1: Introduction}

Aerosols, defined as suspensions of fine solid particles or liquid droplets in a gas, are very common in our daily life. For example, they are present in smoke, oceanic haze, particulate and condensed forms of air pollution, and sprays. A subclass of dispersed particle-laden multiphase flows, research on aerosols is foundationally important to many engineering applications that are related to aerospace, atmospheric, mechanical, biological, chemical, civil, and/or nuclear systems.

The deposition and clearance of inhaled aerosol in the respiratory tract (nasal cavity, mouth-throat, larynx, and lung) are important to human health (Finlay, 2001). This issue continues to be an intense subject of research with specific applications such as prevention and control of respiratory diseases, as well as development of nasal drug delivery, which is the eventual target application of the present work. Nasal drug delivery is a promising alternative to oral and injection routes of medication administration. For example, diabetes is a medical condition in which the body does not produce or use insulin, leading to dangerously high levels of glucose in the blood. It is estimated that 20.8 million people in the United States have diabetes -- $7 \%$ of the total population (data from the American Diabetes Association). In 1995, 135 million people worldwide had diabetes and it is expected that this number will rise to at least 300 million by 2025 (Narayan et al., 2003). In the developed world, diabetes is the most significant cause of adult blindness in the non-elderly and the leading cause of non-traumatic amputation in adults. Diabetic nephropathy is the primary disease requiring renal dialysis in the United States (Petni et al., 2006). It can be painful and hard for most of the 
patients with diabetes to tolerate the many daily needles required for glucose measurement and insulin injection. Needle-free insulin delivery is considered a highly desirable and promising approach, and provides rapid and sustainable absorption of insulin (Petni et al., 2006).

The research of airflow patterns and aerosol deposition within the human nasal cavity geometry is the foundation for improving fraction of nasal drug delivery. Also, nasal breathing is the normal mode of inhalation of ambient aerosols for most adults at low to moderate activity levels, and is the only normal mode of inhalation for young infants when they are at rest (Barry and O'Callaghan, 1997; Schuepp, 2004). To determine the amount of nasally inhaled aerosols deposited in the lungs, it is necessary to know the nasal-throat deposition fraction during nasal breathing (Morgan and Monticello, 1990; Subramaniam et al.,2003; Swift, 1981).

For researchers involved with inhaled aerosols in nasal drug delivery and transmission of environmental aerosols and airborne pathogens, the most important task is to make a predictive understanding of aerosol deposition in order to control aerosol deposition in the respiratory tract, to optimize therapeutic effects of pharmaceutical aerosols, or to minimize detrimental influences of unwanted aerosol particles.

The highly complex geometry of the human nasal cavity is not only an obstacle in the research of airflow patterns in nasal cavity and nasal drug delivery, but also raises significant challenges in clinical research in many related fields including nasal surgery (e.g. Adamson, 1987; O’Neil and Tolley, 1998; Rober et al., 1998; Saunders et al. 1999; Minn et al., 2002; Murry et al., 2003; Pang, 2005; Zhao et al., 2006), nasal toxicology (e.g. Yu et al., 1998; Kimbell et al., 2001; Overton et al., 2001; Bennett and Zeman, 
2005; Roberts et al., 2006; Kimbell, 2006; Tian et al., 2007; Schroeter et al., 2008), risk assessment (e.g. Roush, 1979; Wang et al., 2003; Rhomberg and Lerandowski, 2006), disease diagnosis (e.g. Lenders and Pirsig, 1990; Raj et al., 2000; Kramer and Wray, 2000; Suzina et al., 2003; Davis and Eccles, 2004), and olfaction (e.g. Churchill et al., 2004; Damm et al., 2002; Zhao et al., 2004).

\subsection{Nasal drug delivery}

Traditionally, medication in the form of drops or aerosols has been delivered to the nasal cavity in the treatment of allergies and congestion. More recently, mainly due to developments in safer and better tolerated enhancers to improve medication absorption by the nasal mucosa, the nasal tract has been considered for the delivery of vaccines, insulin, and other medications to treat osteoporosis, blood disorders and migraine headaches (Illum, 2000). Several published work (e.g. Sarkar, 1992; Hussain, 1998; Illum, 2000) showed that nasal drug delivery can allow drugs that do not cross the blood brain barrier (BBB) to be delivered to the central nervous system (CNS), thus eliminating the need for systemic delivery and thereby reducing unwanted systemic side effects (Frey, 2002; Dwibhashyam and Nagappa, 2008). This method works because of the unique connection that the nerves involved in sensing odours and chemicals provide between the brain and the external environment (Illum, 2000).

Compared with other drug delivery methods such as intravenous (IV) infusion, oral and injection routes of delivery, nasal drug delivery has considerable advantages. Some 
of these are as follows (Minn et al., 2002; Hussain, 1998; Mathison et al., 1998; Frey, 2002; Talegaonkar and Mishra, 2004):

- Nasal drug delivery is needle-free, pain-free and comfortable;

- The nasal mucosa has rich vasculature, with a highly permeable structure that enables easy accessibility to blood capillaries greatly enhancing drug absorption;

- Nasal drug delivery can bypass the blood brain barrier (BBB) and target the central nervous system (CNS), reducing systemic exposure and thus systemic side effects;

- Nasal drug delivery avoids the modification of the medical agent which is delivered;

- Nasal drug delivery circumvents the problem of degradation of peptide drugs to a certain extent; and

- Nasal drug delivery allows high, reliable bioavailability because of avoidance of destruction in the gastrointestinal tract, hepatic "first pass" elimination and gut wall metabolism.

Because of its considerable advantages, nasal drug delivery is widely used in many applications including delivery of protein therapeutic agents / macromolecules to the CNS, delivery of DNA plasmids to the CNS, and delivery of small molecules to the CNS (Illum, 2000; Mathison et al., 1998; Hussain et al., 1998, Patni et al., 2006).

\subsection{Nasal anatomy and respiratory physiology}

To study the mechanism of nasal drug delivery, a brief description of the nasal physiology 
is necessary. As an interface between the body and the external world and the first part of human respiratory system, the nose and associated structures perform very important functions concerned with (Marieb, 1995, Grothberg, 2001):

(1) Conditioning inhaled air (warming and/or humidifying);

(2) Filtering inhaled air and cleaning it of foreign matter;

(3) Serving as a resonating chamber for speech; and

(4) Housing the olfactory (smell) receptors.

While the human nasal cavity is highly variable among individuals, it does retain several key features. The nasal cavity is nominally symmetric and is separated into two distinct air passages by a vertical thin wall called the nasal septum. The top part of the nasal cavity is formed by bones and cartilage and is tent shaped. The floor of the nasal cavity is formed by the palate, which separates the nasal and oral cavities and extends horizontally toward the posterior of the skull. As shown in Figure 1.1a, there are three shelves on the lateral wall known as turbinates or conchae, which serve to increase the surface area exposed to the air, thus increasing heat and moisture exchange. The grooved space below each turbinate is referred to as a meatus. The turbinates and meatuses are each labelled superior, middle, or inferior (see Figure 1.1b).

In the nasal vestibules, the regions just after the nostrils, numerous filtering hair follicles and sebaceous glands are present (Rajagopal and Jerry, 2005). Nasal hairs, or vibrissae, consist of the usually small hairs that are in the nose, especially those near the nostrils. Biologically, the hairs act as a filter and keep dust and dirt out of the nasal passages. They also help to maintain humidity in the air passages by trapping humidity 
during exhalation, which then re-evaporates during inhalation to humidify inhaled air (Marieb, 1995).

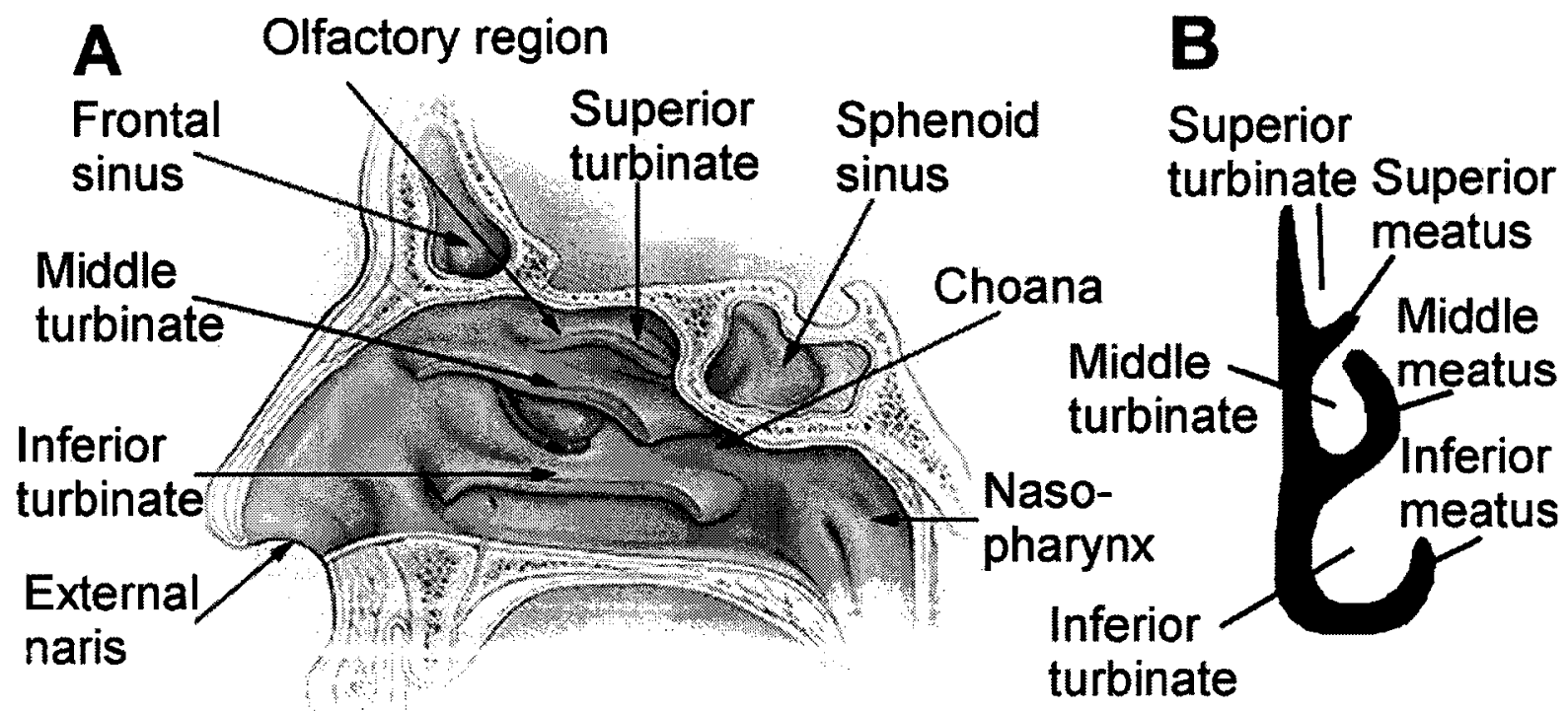

Figure 1.1 Anatomical illustration of the human nasal cavity of a sagittal plane cut (a) and sample coronal plane midway through the nasal cavity (b)

In the superior region of the nasal cavity, the walls are covered by olfactory mucosa. The remainder of the nose is lined with respiratory mucosa (containing ciliated epithelium, producing mucus and secreting sticky mucus containing lysozyme and antibacterial enzymes), which clears the cavity by moving contaminated mucus towards the pharynx (throat), where it is swallowed (Marieb, 1995). As a human breathes, inhaled air enters the nasal cavity from the nostril and passes through the narrowest portion of the nasal cavity, i.e. the nasal valve, which increases its speed (Fishman and Geiger, 1985; Fidone and Gonzalez, 1986). The air then expands and decelerates while flowing through the middle and posterior part of the nasal cavity, impinging and 
circumventing the three turbinates, and potentially creating turbulence that promotes contact between the air and the mucosa. With this complex geometry, the nose filters, warms and humidifies the entering air.

Table1.1 shows a fluid dynamic description of human respiration summarized by Tang et al., (2004). As reported by Bilgen et al. (1974) and Tang et al. (2004), during normal breathing, such as in relaxed, resting and sleeping conditions, the inhalation and exhalation flow rate is usually $7.5-12 \mathrm{~L} / \mathrm{min}$ (or $125-200 \mathrm{~mL} / \mathrm{s}$ ) and the main flow features are periodic and laminar flow. In non-normal conditions, such as exertion, sickness (asthma, nasal polyp, heavy vibrissae, sneezing, etc.) and physical exercise, the inhalation and exhalation flow rate can reach approximately $12-38 \mathrm{~L} / \mathrm{min}(200-625 \mathrm{~mL} / \mathrm{s})$ (Robert, 2001) and the main forms of flow include constriction flows, obstructed flows, extra mucus flows and turbulent flows. A higher flow rate, $150 \mathrm{~L} / \mathrm{min}(2500 \mathrm{~mL} / \mathrm{s})$, can be reached for some specific conditions such as forced inhalation or extremely deep breathing (Hooper, 2001). In polluted environments (where air may contain inorganic, organic, and/or synthetic material dust as well as chemical gas) or during drug delivery, more complexity is added to the main flow features, and aerosol flows, free surface flows, permeation flow and biological reactions may be present. 
Table 1.1 Fluid dynamic description of human respiration (as summarized by Tang et al., 2004).

\begin{tabular}{|c|c|c|c|c|c|}
\hline & $\begin{array}{l}\text { Breathing } \\
\text { conditions }\end{array}$ & $\begin{array}{c}\text { Behaviour } \\
\text { \&Symptoms }\end{array}$ & $\begin{array}{c}\text { Related } \\
\text { locations }\end{array}$ & Main flow features & Simulation approaches \\
\hline 1 & $\begin{array}{l}\text { Normal: } \\
\text {-quiet } \\
\text {-resting } \\
\text {-sleeping }\end{array}$ & $\begin{array}{l}\text { Inspiration } \\
\text { Expiration } \\
\text { Flow rate of 125- } \\
200 \mathrm{~mL} / \mathrm{s}\end{array}$ & \multirow{3}{*}{$\begin{array}{l}\text { a. nasal } \\
\text { cavity } \\
\text { b. larynx } \\
\text { c. trachea } \\
\text { d. bronchi }\end{array}$} & $\begin{array}{l}\text { Periodic flow, } \\
\text { Laminar flow, } \\
\text { Gap flow, small } \\
\text { deformation at the } \\
\text { junction of } b \text { and } c .\end{array}$ & $\begin{array}{c}\text { Sinusoidal velocity } \\
\text { profiles, } \\
\text { single phase approach }\end{array}$ \\
\hline \multirow{3}{*}{2} & \multirow{3}{*}{$\begin{array}{l}\text { Abnormal: } \\
\text {-exertion } \\
\text {-sickness } \\
\text {-physical } \\
\text { exercise }\end{array}$} & $\begin{array}{l}\text {-asthma } \\
\text {-nasal polyp } \\
\text {-heavy vibrissae } \\
\text {-foreign-body } \\
\text {-sneezing } \\
\text { Flow rate of 200- } \\
700 \mathrm{~mL} / \mathrm{s}\end{array}$ & & $\begin{array}{l}\text { constriction flow, } \\
\text { obstruction flow, } \\
\text { extra mucus flow, } \\
\text { high flow rate, } \\
\text { turbulent flow, } \\
\text { deformation at the } \\
\text { conjunction of a to d. }\end{array}$ & $\begin{array}{l}\text { single phase approach, } \\
\text { multiphase approach, } \\
\text { turbulent model } \\
\text { free surface model, } \\
\text { Fluid-Structure } \\
\text { Interaction } \\
\text { (FSI) approach, }\end{array}$ \\
\hline & & -morbid surface & & $\begin{array}{c}\text { excessive } \\
\text { dry/secretion of } \\
\text { the mucosal surface } \\
\text { permeation flow }\end{array}$ & $\begin{array}{c}\text { liquid film flow model } \\
\text { specific boundary model } \\
\text { porous media model }\end{array}$ \\
\hline & & $\begin{array}{l}\text {-cough } \\
\text {-snore } \\
\text {-yawn }\end{array}$ & $\begin{array}{l}\text { a-d. } \\
\text { e. mouth }\end{array}$ & $\begin{array}{l}\text { high flow rate with } \\
\text { deformations and } \\
\text { vibrations in } \mathrm{c} \text { or } \mathrm{d}-\mathrm{e}\end{array}$ & $\begin{array}{l}\text { FSI approach, } \\
\text { acoustic approach }\end{array}$ \\
\hline 3 & $\begin{array}{c}\text { Drug delivery } \\
\text {-inhale } \\
\text {-collunarium }\end{array}$ & Therapy spray & \multirow{2}{*}{$\begin{array}{l}\text { a. nasal } \\
\text { cavity } \\
\text { b. larynx } \\
\text { c. trachea } \\
\text { d. bronchi }\end{array}$} & \multirow{2}{*}{$\begin{array}{c}\text { Aerosol flow, } \\
\text { Droplet flow, } \\
\text { Free surface flow, } \\
\text { Permeation flow } \\
\text { Biological reaction }\end{array}$} & \multirow[b]{2}{*}{$\begin{array}{l}\text { Multiphase approach, } \\
\text { FSI approach, } \\
\text { Porous media model }\end{array}$} \\
\hline 4 & $\begin{array}{l}\text { Air pollution: } \\
\text { - inorganic, } \\
\text { organic, or } \\
\text { synthetic } \\
\text { material dust } \\
\text { - chemical gas }\end{array}$ & $\begin{array}{c}\text { Silicosis } \\
\text { Asbestosis, } \\
\text { Chronic obstructive } \\
\text { Pulmonary disease }\end{array}$ & & & \\
\hline
\end{tabular}

\subsection{Two-phase flow and mechanisms of particle deposition}

Two-phase flow entails fluid dynamic motion of various phases. Particle components of a two-phase flow can be solid, liquid, or gas, while the surrounding fluid must be a liquid or a gas (Crowe, 2005). Two-phase flows can also be categorized by concentration. Volume fractions of $0.1 \%$ or even less lead to "sparse" flows, where particle concentrations are so low that both particle-particle interactions and the effect of particles on the surrounding fluid are negligible (Elgobashi, 1994, Crowe, 2005). In this case, 
only the continuous-fluid affects particle motion, and the interaction between the particles and surrounding flow is called as one-way coupling. When the particle volume fractions are less than $10 \%$ and particles sizes are small compared to that of the overall domain, two-phase flows are considered "dispersed" (Loth, 2009). In this case, the changes in particles trajectories are dominated by their interaction with the surrounding fluid, and the particle motion is chiefly governed by particle inertia and the fluid dynamic surface forces (e.g., drag force) and body forces (primarily gravitational). This interaction is called two-way coupling. For volume fractions greater than $10 \%$, flows are referred to as "dense" two-phase flows, where particle movement is dominated by particle-particle interactions, e.g. by collisions or contact mechanics (Elgobashi, 1994) and this regime is termed four-way coupling.

Research of particle-laden two-phase flow is fundamental to many engineering applications including energy systems (e.g. circulating fluidized beds), propulsion (e.g. rocket engines), atmospheric dispersion (e.g. models of the concentration distribution of micron and sub-micron sized solid and liquid particles in the atmosphere) and biological flows (e.g. particle motion in the blood stream and the body, nasal drug delivery), to list just a few. Table 1.2 summarizes common two-phase flows in various engineering systems (Loth, 2009).

Table 1.2 Two-phase flow in various engineering systems, summarized by Loth (2009)

\begin{tabular}{|c|c|}
\hline Two-Phase Flows & Engineering Application \\
\hline Solid particles in a gas & fluidized beds, solid rockets, particle separators, ventilation ducts \\
\hline Liquid droplets in a gas & $\begin{array}{c}\text { atmospheric analysis, liquid fuel sprays, nasal or oral drug delivery } \\
\text { devices }\end{array}$ \\
\hline Solid particles in a liquid & liquid filters, sedimentation, solidification, slurries \\
\hline Gas bubbles in a liquid & naval vessels, bubble columns, molten metal baths, boilers \\
\hline
\end{tabular}




\subsubsection{Particle Deposition}

There are five basic mechanisms by which an aerosol particle can be deposited on a wall or a fibre (Hinds, 1982; Guha, 2008): inertial impaction, diffusion, gravitational settling, interception and electrostatic deposition. The most important mechanisms for inhalation particle deposition are inertial impaction, sedimentation, and diffusion (Hinds, 1982).

(1) Inertial impaction

Inertial impaction of a particle on a fibre (for examples, nasal hairs for inhaled particles) (or even an adjacent wall) occurs when a particle, due to its inertia, is incapable of adjusting quickly enough to the suddenly changing streamlines in the vicinity of a fibre and crosses those streamlines to hit the wall or the fibre. Inertial impaction is a major deposition mechanisms for aerosol particles bigger than $1 \mu \mathrm{m}$ (Tien and Ramarao, 2007). During inhalation, the directions of inhaled air change several times as it flows from the nose or mouth down through the branching airway system (Hinds, 1982). Each time the air changes direction, the suspended particles tend to keep moving in their original direction for short distance because of their inertia. When the particles diverge from the corresponding streamlines, some of them may impact on the airway surface, which leads to particle deposition.

(2) Diffusion

For small particles (less than $1 \mu \mathrm{m}$ in size), Brownian diffusion and turbulent diffusion are sufficiently important to greatly enhance the possibility of the particles hitting a fibre while traveling past it on a non-intercepting streamline (Friedlander and Johnstone 1957; McCoy and Hanratty 1977). This is most likely to occur in smaller 
airways where distances are short and residence times are comparatively long. Diffusion is the predominant deposition mechanism for particles smaller than $0.5 \mu \mathrm{m}$ (Schulz et al., 2000), and is governed by geometric rather than aerodynamic size, which means the density of particle is not an important factor for this mechanism. Gehr and Heyder (2000) pointed out that since smaller particles tend to reside longer in the respiratory system, the probability of smaller particles hitting an airway surface by diffusion is increased.

(3) Gravitational settling

Gravitational setting (also called sedimentation) is the most important deposition mechanism in the smaller airways and the alveoli where airflow velocities are low and airway dimensions are small (Schulz et al., 2000). Since the driving force for deposition via this mechanism is due to gravity, sedimentation has its maximum removal effect in airways that are oriented horizontally. In contrast with diffusional transport, gravitational settling is significant for the deposition of particles larger than $0.5 \mu m$ (Schulz et al., 2000). Also, the distance a particle settles within a given time increases with its density. For inhaled aerosol particles, the longer a particle remains in the respiratory airway, the larger the settling distance the particle can cover, and hence, the larger the probability that the particle can hit on the airway surface. Hygroscopic particles (particles that preferentially absorb water) grow as they pass through the water-saturated airways, and this increase in size favours deposition by sedimentation and impaction in the distal airway (Hinds, 1982). 
(4) Interception

Particle deposition by interception occurs specifically when a particle follows a gas streamline that happens to come within one particle radius of the surface of a wall or a fibre. This mechanism is not very important in deposition of inhaled particles (Hinds, 1982). The probability of interception depends on the proximity of the gas streamline to the airway surface and on the ratio of particle size to airway diameter, which is usually small even in the smallest airways. Generally, interception is an important deposition mechanism only if the inertial impaction effect is negligible (Tien and Ramarao, 2007).

(5) Electrostatic attraction

Although most ambient aerosol particles are neutralized by air ions, many freshly generated particles often carry electrostatic charges which may affect their deposition. Highly charged particles are attracted to airway surfaces by the electrostatic image charges generated by the presence of these charged particles and thus, may have an enhanced deposition ( $\mathrm{Yu}, 1985)$.

\subsection{Focus of the current research}

Aerosol particle distributions and deposition patterns in the nasal cavity are critical parameters affecting the fraction of nasal absorption. To optimize the delivery of therapeutic aerosols, a full understanding of airflow patterns and particle deposition patterns in the human nasal cavity is necessary through experimental (both in vitro and in vivo) and numerical studies. The deposition of particles in the nasal cavity is a function of particle size and respiratory patterns. The density, shape, and hygroscopicity of 
particles, the pathological conditions in the nasal passage and the inhalation flow rate all influence the deposition of particles.

Although several previous researchers have investigated deposition of inhaled aerosols for both oral and nasal breathing during the past decades (e.g. Swift, 1991; Lennon et al., 1998; Cheng et al., 1988; Zwartz and Guilmette, 2001; Cheng et al., 2001; Janssens et al., 2001; Kelly et al., 2004; Garcia et al., 2009), to the author's knowledge, none have done so using a standardized geometry and very few attempts have been made to quantitatively characterize regional deposition of inhaled particles in the nasal cavity. One part of present work was to create a standardized three-dimensional geometry of the human nasal cavity representative of the general population that could be used for a variety of applications including research on nasal drug delivery and deposition. Although several reports related to the measurement of characteristic dimensions of the human nasal cavity and generation of human nasal cavity models have appeared in the literature (see detailed discussion in section 2.1), prior to the current work, the creation/construction of a median human nasal cavity geometry had not been attempted and accomplished.

Using the novel standardized human nasal cavity geometry, numerical simulations and experimental (in vitro) measurements of aerosol particle deposition patterns and airflow patterns were performed. Results from this work offer detailed information of aerosol particle deposition, including regional deposition patterns, which are important factors affecting the fraction of nasal absorption, and are important for advancing nasal drug delivery technology.

Specific goals of the research presented in this thesis were to: 
1. Summarize geometric characteristics of the nasal cavities of 30 healthy adults by processing computed tomography (CT) scans offered by the Ottawa Hospital, Civic campus; develop an objective methodology for aligning, scaling, and averaging the associated 60 single individual nasal passages; and use this methodology to create a single, detailed, realistic, standardized geometry that could be considered as a standard human nasal cavity for in vitro aerosol deposition studies and numerical simulation;

2. Design a particle deposition apparatus and conduct experiments to measure total and regional particle deposition fraction using the standardized nasal geometry;

3. Simulate airflow patterns and particle deposition in the standardized human nasal cavity geometry using Reynolds Averaged Navier-Stokes equations/Eddy-interaction model (RANS/EIM) and standard Large Eddy Simulation (LES), to better understand the limits of applicability and accuracy of standard numerical methods (mostly available in commercial packages);

4. Investigate influences of particle size and air flow rate on particle deposition patterns;

5. Improve understanding of dispersed particle-laden flows within the geometrically complex human nasal cavity;

6. Investigate influences of nasal geometry (especially the size of nostril) on airflow patterns and particle deposition patterns. 


\subsection{Thesis Organization}

The thesis consists of 8 chapters. Chapter 1 summarized the motivation, background and aims of current research. In Chapter 2, a literature review concerning airflow patterns and particle deposition fraction in human nasal cavity is presented. The construction of the "Civic-Carleton" novel standardized human nasal cavity geometry is described in Chapter 3. In Chapter 4 the experimental apparatus and method used to measure the total and regional particle deposition fraction in the novel standardized human nasal cavity are described. Chapter 5 presents the discussion of measured total and regional particle deposition fraction in the novel standardized human nasal cavity. Chapter 6 describes the numerical simulation methodology adopted in present research. In Chapter 7, grid convergence and sensitivity analyses are performed, and simulation results of total particle deposition as well as simulated airflow patterns in the standardized human nasal cavity are presented and discussed. Chapter 7 also discusses the numerical and experimental results of regional particle deposition fraction. Finally, conclusions are summarized in Chapter 8 along with recommendations for future work. 


\section{Chapter 2: $\quad$ Literature Review}

A better understanding of deposition of inhaled aerosol within the complex human respiratory system (nasal cavity, oral cavity and respiratory tract) is important in developing pharmaceutical aerosols for drug delivery, as well as in preventing and controlling the health risks associated with environmental aerosols and bio-aerosols. Although several studies of deposition of inhaled aerosols for both oral and nasal breathing have been conducted during the past few decades, for the most part these have used specific nasal geometries and in many cases these have been limited by a lack of a standardized or generalized geometry representative of the broader population. In this chapter, a brief review of previous investigations of human nasal cavity geometry, airflow patterns, and particle deposition fraction is presented.

\subsection{Previous Measurements of characteristic dimensions in human nasal cavities}

Many attempts have been made to measure characteristic dimensions of the human nasal cavity to create a realistic human nasal cavity 3D model. Data on characteristic dimensions of the human nasal cavity (cross-sectional area, surface area, volume and nostril shape) is the foundation of experimental and numerical studies of airflow patterns and particle deposition patterns.

As discussed in Section 1.2, the human nasal cavity is highly complex and variable among individuals, which not only makes the study of particle deposition in nasal cavity 
difficult, but also raises significant challenges in clinical research in many related fields. Fortunately, advanced technology such as computed tomography (CT), acoustic rhinometry (AR) and magnetic resonance imaging (MRI) make it possible to gather detailed information on the dimensions of the human nasal cavity. Table 2.1 summarizes the measurements taken in several previous studies along with number of subjects and techniques used.

Table 2.1 Summarized measurements of characteristic dimensions of human nasal cavities

\begin{tabular}{|c|c|c|c|c|}
\hline $\begin{array}{c}\text { Researchers and } \\
\text { year }\end{array}$ & $\begin{array}{l}\text { Number, } \\
\text { gender (M/F) } \\
\text { and age of } \\
\text { subjects }\end{array}$ & Method & Reported Measurement Data & Focus of paper \\
\hline $\begin{array}{l}\text { Hilberg et al., } \\
1989\end{array}$ & $\begin{array}{c}6,3 / 3 \\
22 \sim 55 \text { years }\end{array}$ & AR & $\begin{array}{l}\text { Cross-sectional area as a } \\
\text { function of distance for both left } \\
\text { and right nasal cavities }\end{array}$ & $\begin{array}{l}\text { Assessed AR technique for } \\
\text { measuring cross sectional area of } \\
\text { the nasal cavity as a function of the } \\
\text { distance from the nostril }\end{array}$ \\
\hline Min et al., 1995 & $\begin{array}{l}30,22 / 8 \\
20 \sim 62 \text { years }\end{array}$ & $\begin{array}{l}\text { AR and } \\
\text { CT scans }\end{array}$ & $\begin{array}{l}\text { Cross-sectional area as a } \\
\text { function of distance for both left } \\
\text { and right nasal cavities }\end{array}$ & $\begin{array}{l}\text { Evaluated the validity and } \\
\text { characteristics of } A R \text { tests compared } \\
\text { with the results obtained from CT } \\
\text { scans }\end{array}$ \\
\hline $\begin{array}{l}\text { Swift and } \\
\text { Kesavanathan, } \\
1996\end{array}$ & $\begin{array}{l}1,1 / 0 \\
\text { N/A }\end{array}$ & $\begin{array}{l}\text { In vivo } \\
\text { casting }\end{array}$ & $\begin{array}{l}\text { Cross-sectional area, perimeter, } \\
\text { and hydraulic diameters of } 11 \\
\text { cross sections }\end{array}$ & $\begin{array}{l}\text { Used fibrous filter theory to } \\
\text { determine the deposition fraction in } \\
\text { nasal cavity }\end{array}$ \\
\hline $\begin{array}{l}\text { Cheng, Y. S. et } \\
\text { al., } 1996\end{array}$ & $\begin{array}{c}4,4 / 0 \\
36 \sim 57 \text { years }\end{array}$ & MRI & $\begin{array}{c}\text { Cross-sectional area, total } \\
\text { surface area, and perimeter of } \\
\text { cross sections }\end{array}$ & $\begin{array}{c}\text { Experiments on deposition of } \\
\text { ultrafine particles in human nasal } \\
\text { cavity and its relationship to airway } \\
\text { geometry }\end{array}$ \\
\hline $\begin{array}{l}\text { Cheng, K. H. et } \\
\text { al., } 1996\end{array}$ & $\begin{array}{c}10,10 / 0 \\
24 \sim 58 \text { years }\end{array}$ & $\begin{array}{l}\text { MRI and } \\
\text { AR }\end{array}$ & $\begin{array}{l}\text { Minimum cross-sectional area, } \\
\text { surface area, and defined "shape } \\
\text { complexity" }\end{array}$ & $\begin{array}{l}\text { Deposition fraction in terms of nasal } \\
\text { shape parameters }\end{array}$ \\
\hline Corey et al., 1997 & $\begin{array}{l}5,3 / 2 \\
\text { N/A }\end{array}$ & $\begin{array}{l}\text { AR and } \\
\text { MRI }\end{array}$ & $\begin{array}{l}\text { Cross-sectional area and volume } \\
\text { as a function of distance }\end{array}$ & $\begin{array}{l}\text { Determine accuracy of AR as a } \\
\text { technique in assessing nasal CSAs } \\
\text { and volumes by comparing with MRI }\end{array}$ \\
\hline $\begin{array}{l}\text { Kesavanathan } \\
\text { and Swift, } 1998\end{array}$ & $\begin{array}{c}10,7 / 3 \\
23 \sim 60 \text { years }\end{array}$ & AR & $\begin{array}{c}\text { Nostril shape (characteristic } \\
\text { dimensions) }\end{array}$ & $\begin{array}{c}\text { Effect of particle size, flow rate, } \\
\text { nostril shape and nasal passage } \\
\text { geometry on nasal particle } \\
\text { deposition fraction }\end{array}$ \\
\hline $\begin{array}{l}\text { Terheyden et al. } \\
\qquad 2000\end{array}$ & $\begin{array}{c}6,3 / 3 \\
22 \sim 55 \text { years }\end{array}$ & $\begin{array}{l}\text { CT scans } \\
\text { and AR }\end{array}$ & $\begin{array}{l}\text { Comparison of CSA-distance } \\
\text { curve using CT scans and AR }\end{array}$ & $\begin{array}{l}\text { Validate the AR technique against CT } \\
\text { scanning }\end{array}$ \\
\hline
\end{tabular}




\subsubsection{Measurements Using Acoustic rhinometry}

Acoustic rhinometry (AR) is based on reflections of an acoustic pulse applied to the nostrils. From the reflected wave, the serial distribution of impedance can be calculated as a function of traveling time (Jackson et al., 1977). Because acoustic impedance depends on the cross-sectional area, the result is presented as a serial distribution of cross sections of an acoustic system equivalent to the acoustic properties of the nose (Jackson et al., 1977). This method has many advantages: it is noninvasive, it requires little cooperation of the subject, and it is applicable in small children (Buenting et al., 1994). Thus, AR has been widely used to make in vivo cross-sectional measurements of the human trachea and the lower airways (Jackson et al., 1977; Fouke and Strohl, 1987). In 1989, the method was introduced for assessment of the patency of the nose (Hilberg et al., 1989).

Several investigations have been performed in the last decades to measure basic characteristic dimensions of the human nasal cavity using AR including Cheng $\mathrm{KH}$ et al., (1996); Corey et al., (1997); Hilberg et al., (1989); Kesavanathan and Swift (1998); Min and Jang (1995); and Terheyden et al., (2000). Kesavanathan and Swift (1998) measured the characteristic dimensions of the nostrils of ten healthy Asian and European American subjects aged 23-60 years using AR. Three nostril shape measurements were taken: the major axis of the nostril cross-section (the length of the nostril), the minor axis of the nostril cross-section (the width of the nostril), and the angle of the major axis with the base of the nose. Several of the other studies listed (e.g Hilberg et al., 1989; Min and Jang, 1995; Reber, 1998; Terheyden et al., 2000; Damm et al., 2002;) were primarily 
concerned with demonstrating the acoustic rhinometry technique for estimating flow cross-sectional areas and comparing its accuracy with more precise MRI and CT measurements. As noted by Terheyden et al. (2000), AR is better used as diagnostic tool for the anterior parts of the nose, since the measurement accuracy of AR in the posterior nasal cavity is unsatisfactory.

\subsubsection{Nasal Cavity Measurements using Computed Tomography and Magnetic Resonance Imaging}

Computed tomography (CT) is a medical imaging method using X-rays to obtain images, and is widely used in the medical field (to create cross-sectional pictures of the body). Magnetic resonance imaging (MRI) is similar to $\mathrm{CT}$ but uses non-ionizing radio frequency signals to acquire images and can provide much greater contrast between the different soft tissues of the body than CT. Compared with CT, MRI is a relatively new technology whose first image was published in 1973 (Lauterbur, 1973; Hinshaw et al., 1973), and the first published studies performed on the human body appeared in 1977 (Damadian et al., 1977).

Rapid developments in medical imaging (CT and MRI scanning) coupled with computer science have made it possible to obtain detailed geometric information of nasal cavity (Min and Jang, 1995; Cheng KH et al., 1996; Corey et al., 1997; Terheyden et al., 2000; Cheng YS et al., 2003).

As shown in Figure 2.1, published measured results of cross sectional areas of the human nasal cavity using AR, CT or MRI scanning span a wide range, indicating the 
non-negligible individual differences among human nasal geometries. This illustrates the importance of generating a standardized 3D human nasal cavity which can eliminate the significant individual differences while representing the general population. In present work, CT data were used to create a standardized 3D human nasal cavity model. Detailed analysis and comparison of the dimensions of the standardized 3D human nasal cavity model with previous data will be presented in Chapter 3 .

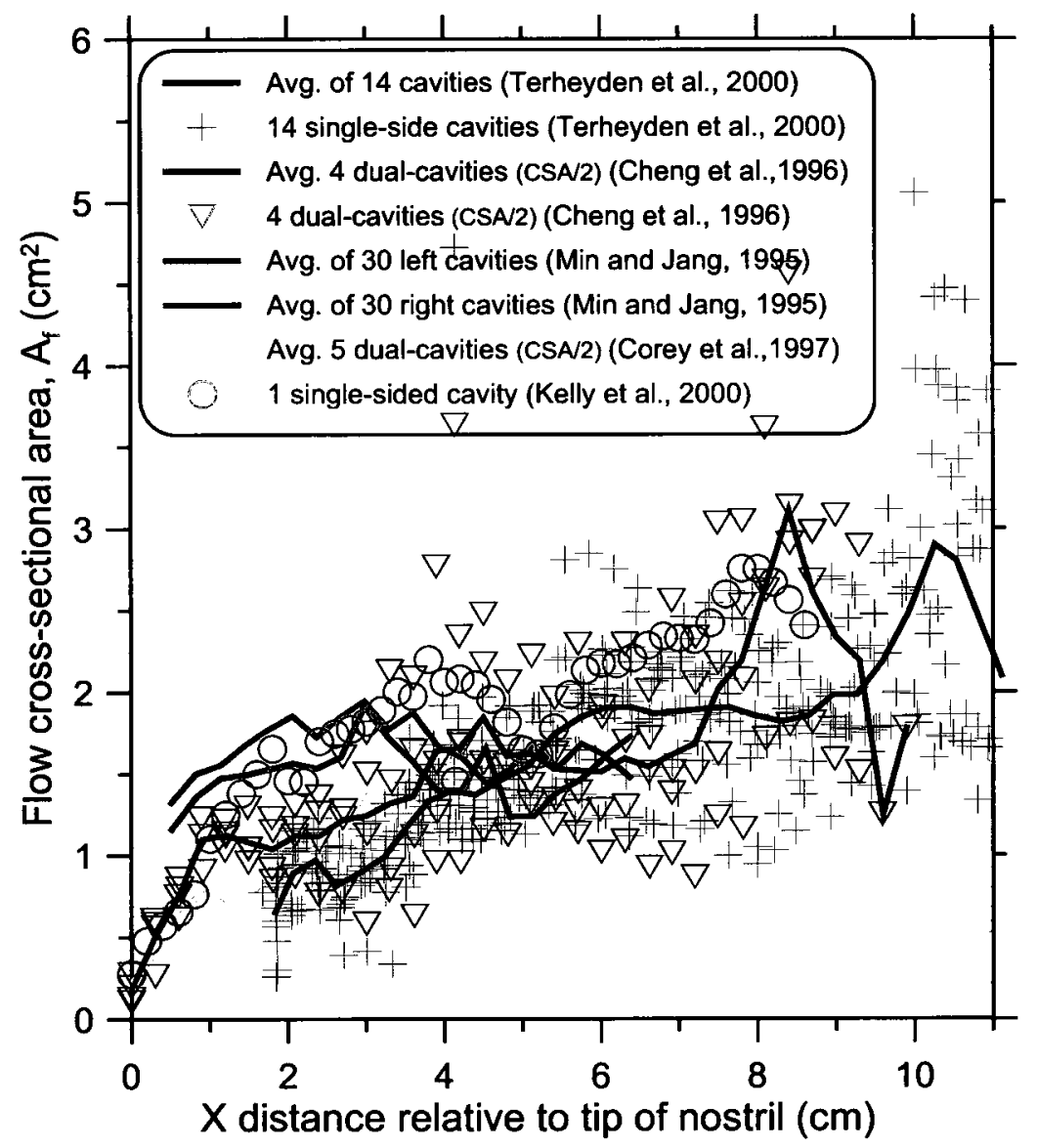

Figure 2.1 Cross-sectional areas of nasal models (green curve) derived from published data of CT scans and MRI scans 


\subsection{Published 3D human nasal cavity models}

Several realistic 3D models of the human nasal cavity have been generated using magnetic resonance imaging (MRI) (Swift 1991; Schreck et al., 1993; Guilmette and Gagliano, 1994; Zwartz and Guilmette, 1999; Shanley et al., 2008; Garcia et al., 2009) and computed tomography (CT) (Lennon, 1998; Kelly et al., 2000, Inthavong et al., 2006). These are summarized in Table 2.2 .

It can be seen from Table 2.2 that, there are only nine different subjects $(7$ males $/ 2$ females) were used to generate replica or 3D models of nasal cavities. Sometimes, a set of MRI or CT scan was used by different researchers to generate physical 3D replicas using different manufacturing methods. For example, six replicas were generated to perform particle deposition experiments using the same set of MRI scans of nasal cavity of a 53-year-old non-smoking Caucasian male (Swift, 1991; Guilmette et al., 1994; Zwartz \& Guilmette, 2001; Kelly et al., 2004; Garcia et al., 2009), and Shi et al. also used this set of MRI scans to generate 3D model to simulate inertial particle transport and deposition in human nasal cavities.

Among these nine subjects, age information of four of them was provided (25/25/53/59-year-old), and all these four subjects were healthy male. For body mass, information of five out of nine subjects was provided (Swift, 1991; Inthanvong et al., 2006; Replica 2, 3, and 4 of Garcia et al., 2009): varied from $55.3 \mathrm{~kg}$ to $74 \mathrm{~kg}$. About gender information, the ratio of male to female was seven to two. Only four subjects had specific ethnic information: two Caucasian (Swift, 1991; Guilmette \& Gagliano, 1994) and two Asian (Kim \& Chung, 2003; Inthanvong et al., 2006), who were all male. 
In summary, even though several 3D nasal cavity models have been generated and have been used by different researchers to perform experiments and numerical simulations, none of them can be used as a median or standardized model to represent the general population because all previous models were generated from data source of single subject. Another limitation of previous subjects is lacking of physiological and ethnic diversity. 
Table 2.2 Summary of 3D human nasal cavity models

\begin{tabular}{|c|c|c|c|c|c|c|c|c|}
\hline $\begin{array}{c}\text { Researchers } \\
\text { and year }\end{array}$ & $\begin{array}{l}\text { Data source } \\
\text { and } \\
\text { information } \\
\text { of subjects }\end{array}$ & $\begin{array}{c}\text { Scan } \\
\text { resolution }\end{array}$ & $\begin{array}{c}\text { Outlining } \\
\text { method }\end{array}$ & $\begin{array}{l}\text { Manufacturing } \\
\text { methods }\end{array}$ & $\begin{array}{l}\text { Scale } \\
\text { ratio }\end{array}$ & $\begin{array}{c}\text { Manufacturing } \\
\text { resolution } \\
(\mathbf{m m})\end{array}$ & $\begin{array}{c}\text { Measurements } \\
\text { or } \\
\text { simulations }\end{array}$ & $\begin{array}{l}\text { Use in other } \\
\text { studies }\end{array}$ \\
\hline Swift, 1991 & $\begin{array}{c}\text { MRI, a 53- } \\
\text { year-old non- } \\
\text { smoking } \\
\text { Caucasian } \\
\text { male } \\
(73 \mathrm{~kg})\end{array}$ & $\begin{array}{c}3 \mathrm{~mm} \\
\text { (coronol) }\end{array}$ & $\begin{array}{c}\text { Traced by } \\
\text { hand }\end{array}$ & $\begin{array}{l}\text { Carving coronal } \\
\text { plates by hand } \\
\text { with high-speed } \\
\text { cutting tool }\end{array}$ & 1 & $N / A$ & $\begin{array}{c}\text { Experiments on } \\
\text { particle deposition }\end{array}$ & $\begin{array}{l}\text { Experiments } \\
\text { (Guilmette et } \\
\text { al., 1994; } \\
\text { Zwartz \& } \\
\text { Guilmette, } \\
\text { 2001; Kelly et } \\
\text { al., 2004; } \\
\text { Garcia et al., } \\
\text { 2009) } \\
\text { Simulations } \\
\text { (Shi et al., } \\
\text { 2007) }\end{array}$ \\
\hline $\begin{array}{l}\text { Guilmette et } \\
\text { al.,1994 }\end{array}$ & $\begin{array}{l}\text { Same as } \\
\text { Swift, } 1991\end{array}$ & $\begin{array}{c}3 \mathrm{~mm} \\
\text { (coronal) }\end{array}$ & \begin{tabular}{|} 
Digitized \\
using \\
CAD \\
software \\
\end{tabular} & $\begin{array}{l}\text { Computerized } \\
\text { milling machine }\end{array}$ & 1 & $\begin{array}{c}1.5 \text { (coronoal) } \\
0.02 \text { (lateral) }\end{array}$ & $\begin{array}{c}\text { Experiments on } \\
\text { particle deposition }\end{array}$ & N/A \\
\hline $\begin{array}{c}\text { Zwartz \& } \\
\text { Guilmette, } \\
2001\end{array}$ & $\begin{array}{l}\text { Same as } \\
\text { Swift, } 1991\end{array}$ & $\begin{array}{c}3 \mathrm{~mm} \\
\text { (coronal) }\end{array}$ & \begin{tabular}{|c|} 
Digitized \\
using \\
CAD \\
software \\
\end{tabular} & $\begin{array}{l}\text { Computerized } \\
\text { milling machine }\end{array}$ & 1 & $\begin{array}{l}1.5 \text { (coronoal) } \\
0.02 \text { (lateral) }\end{array}$ & $\begin{array}{c}\text { Experiments on } \\
\text { particle deposition }\end{array}$ & N/A \\
\hline $\begin{array}{c}\text { Kelly et al., } \\
2004 \\
\text { SLA replica }\end{array}$ & $\begin{array}{l}\text { Same as } \\
\text { Swift, } 1991\end{array}$ & $\begin{array}{c}3 \mathrm{~mm} \\
\text { (coronal) }\end{array}$ & \begin{tabular}{|} 
Digitized \\
using \\
CAD \\
software \\
\end{tabular} & \begin{tabular}{|} 
Stereolithography \\
SLA 7000 (3D \\
Systems, Inc.)
\end{tabular} & 1 & $\begin{array}{l}0.25 \text { (coronal) } \\
0.102 \text { (lateral) }\end{array}$ & $\begin{array}{c}\text { Experiments on } \\
\text { particle deposition }\end{array}$ & N/A \\
\hline \begin{tabular}{c|} 
Kelly et al., \\
2004 \\
Viper replica
\end{tabular} & $\begin{array}{l}\text { Same as } \\
\text { Swift, } 1991\end{array}$ & $\begin{array}{c}3 \mathrm{~mm} \\
\text { (coronol) }\end{array}$ & \begin{tabular}{|c|} 
Digitized \\
using \\
CAD \\
software \\
\end{tabular} & $\begin{array}{l}\text { Stereolithography } \\
\text { Viper Si2 machine } \\
\text { (3D Systems, Inc.) }\end{array}$ & 1 & $\begin{array}{c}0.075 \text { (coronal) } \\
0.051 \text { (lateral) }\end{array}$ & $\begin{array}{c}\text { Experiments on } \\
\text { particle deposition }\end{array}$ & $N / A$ \\
\hline $\begin{array}{c}\text { Garcia et al., } \\
2009 \\
\text { Replica } 1\end{array}$ & $\begin{array}{l}\text { Same as } \\
\text { Swift, } 1991\end{array}$ & $\begin{array}{c}3 \mathrm{~mm} \\
\text { (coronal) }\end{array}$ & \begin{tabular}{|l|} 
Digitized \\
using \\
CAD \\
software \\
\end{tabular} & $\begin{array}{l}\text { Stereolithography } \\
\text { Viper Si2 machine } \\
\text { (3D Systems,Inc.) }\end{array}$ & 1 & $\begin{array}{c}0.075 \text { (coronal) } \\
0.051 \text { (lateral) }\end{array}$ & $\begin{array}{c}\text { Experiments on } \\
\text { particle deposition }\end{array}$ & N/A \\
\hline $\begin{array}{c}\text { Guilmette \& } \\
\text { Gagliano, } \\
1994\end{array}$ & \begin{tabular}{|c} 
MRI, a 59- \\
year-old non- \\
smoking \\
Caucasian \\
male
\end{tabular} & $\begin{array}{c}3 \mathrm{~mm} \\
\text { (coronal) }\end{array}$ & $\begin{array}{l}\text { Digitized } \\
\text { using } \\
\text { CAD } \\
\text { software }\end{array}$ & $\begin{array}{l}\text { Computerized } \\
\text { milling machine }\end{array}$ & 1 & $\begin{array}{l}0.5 \text { (coronal) } \\
0.02 \text { (lateral) }\end{array}$ & $\begin{array}{c}\text { Experiments on } \\
\text { particle deposition }\end{array}$ & $\begin{array}{c}\text { Experiments } \\
\text { (Zwartz \& } \\
\text { Guilmette, } \\
\text { 1999) }\end{array}$ \\
\hline $\begin{array}{c}\text { Zwartz \& } \\
\text { Guilmette, } \\
1999\end{array}$ & $\begin{array}{c}\text { Same as } \\
\text { Guilmette \& } \\
\text { Gagliano, } \\
1994 \\
\end{array}$ & $\begin{array}{c}3 \mathrm{~mm} \\
\text { (coronal) }\end{array}$ & \begin{tabular}{|} 
Digitized \\
using \\
CAD \\
software \\
\end{tabular} & $\begin{array}{l}\text { Computerized } \\
\text { milling machine }\end{array}$ & 1 & $\begin{array}{l}0.5 \text { (coronal) } \\
0.02 \text { (lateral) }\end{array}$ & $\begin{array}{c}\text { Experiments on } \\
\text { particle deposition }\end{array}$ & $N / A$ \\
\hline $\begin{array}{l}\text { Kelly et al., } \\
2000\end{array}$ & $\begin{array}{c}\text { CT scans, a } \\
\text { healthy } 25- \\
\text { year-old man }\end{array}$ & $\begin{array}{l}1-5 \mathrm{~mm} \\
\text { (coronal) }\end{array}$ & \begin{tabular}{|c|} 
Used \\
IDEAS \\
Master \\
Series \\
solid \\
modeling \\
package
\end{tabular} & $\begin{array}{c}\text { 3D printer ( } Z \\
\text { Corporation) and } \\
\text { curing clear silicon } \\
\text { (Dow Corning) }\end{array}$ & 2 & N/A & $\begin{array}{l}\text { PIV experiments } \\
\text { (Velocity) }\end{array}$ & $\begin{array}{c}\text { Experiments } \\
\text { (Hopkins et al., } \\
2000) \\
\text { Simulations } \\
\text { (Liu et } \\
\text { al.,2007) }\end{array}$ \\
\hline $\begin{array}{c}\text { Hopkins et al., } \\
2000\end{array}$ & $\begin{array}{l}\text { Same as } \\
\text { Kelly et al., } \\
2000\end{array}$ & $\begin{array}{l}1-5 \mathrm{~mm} \\
\text { (coronal) }\end{array}$ & \begin{tabular}{|c|} 
Used \\
IDEAS \\
Master \\
Series \\
solid \\
modeling \\
package
\end{tabular} & $\begin{array}{c}\text { 3D printer ( } Z \\
\text { Corporation) and } \\
\text { curing clear silicon } \\
\text { (Dow Corning) }\end{array}$ & 2 & N/A & $\begin{array}{l}\text { PIV experiments } \\
\text { (Velocity)Velocity }\end{array}$ & N/A \\
\hline
\end{tabular}




\begin{tabular}{|c|c|c|c|c|c|c|c|c|}
\hline $\begin{array}{c}\text { Kim \& Chung, } \\
2003\end{array}$ & \begin{tabular}{|c|} 
CT scans, \\
Korean adults \\
with and \\
without \\
adenoid \\
vegetation \\
\end{tabular} & N/A & N/A & $\begin{array}{c}\text { 3D printer ( } Z \\
\text { Corporation) and } \\
\text { curing clear silicon }\end{array}$ & 1 & N/A & $\begin{array}{l}\text { PIV experiments } \\
\text { (Velocity)Velocity }\end{array}$ & $\begin{array}{c}\text { Experiments } \\
\text { (Kim \& Chung } \\
\text { 2004; Kim \& } \\
\text { Haw, 2004) }\end{array}$ \\
\hline $\begin{array}{c}\text { Inthanvong et } \\
\text { al., } 2006\end{array}$ & \begin{tabular}{|} 
CT scans of a \\
25-year-old \\
healthy, non- \\
smoking \\
Asian male \\
$(73 \mathrm{~kg}, 173$ \\
$\mathrm{cm})$
\end{tabular} & $\begin{array}{c}1-5 \mathrm{~mm} \\
\text { (coronal) }\end{array}$ & $\begin{array}{c}\text { 3D } \\
\text { modeling } \\
\text { program } \\
\text { GAMBIT }\end{array}$ & NA & 1 & $\mathrm{~N} / \mathrm{A}$ & $\begin{array}{c}\text { Simulation on } \\
\text { particle deposition }\end{array}$ & $\begin{array}{l}\text { Simulations } \\
\text { (Tian et al., } \\
\text { 2007) }\end{array}$ \\
\hline $\begin{array}{c}\text { Shanley et al., } \\
2008\end{array}$ & $\begin{array}{c}\text { MRI scans of } \\
\text { an } \\
\text { anonymous, } \\
\text { healthy, adult } \\
\text { male human } \\
\text { subject }\end{array}$ & $\begin{array}{c}1-5 \mathrm{~mm} \\
\text { (coronal) }\end{array}$ & $\begin{array}{c}\text { 3D } \\
\text { modeling } \\
\text { program } \\
\text { GAMBIT }\end{array}$ & NA & 1 & $\mathrm{~N} / \mathrm{A}$ & $\begin{array}{c}\text { Simulation on } \\
\text { particle deposition }\end{array}$ & N/A \\
\hline $\begin{array}{l}\text { Garcia et al., } \\
2009 \\
\text { Replica 2, 3, } \\
\text { and 4 }\end{array}$ & $\begin{array}{c}\text { MRI scans of } \\
\text { three healthy } \\
\text { subjects ( } 2 \\
\text { females and } \\
\text { one male) }\end{array}$ & $\begin{array}{c}3 \mathrm{~mm} \\
\text { (coronol) }\end{array}$ & $\begin{array}{l}\text { Digitized } \\
\text { using } \\
\text { CAD } \\
\text { software }\end{array}$ & $\begin{array}{l}\text { Stereolithography } \\
\text { Viper Si2 machine } \\
\text { (3D Systems, Inc.) }\end{array}$ & 1 & $\begin{array}{c}0.075 \text { (coronal) } \\
0.051 \text { (lateral) }\end{array}$ & $\begin{array}{c}\text { Experiments on } \\
\text { particle } \\
\text { depositionParticle } \\
\text { deposition }\end{array}$ & N/A \\
\hline
\end{tabular}




\subsubsection{Methods of Manufacturing Nasal Cavity Replicas}

As summarized in Table 2.2, there are several ways to manufacture replicas of nasal cavities from 3D nasal cavity models: assembly from coronal planes carved by hand; manufacture using different rapid prototyping technologie (sometimes combined with curing clear silicon) such as $3 \mathrm{D}$ printer, stereolithography (SL) and so on.

Swift (1991) generated a replica using MRI scans of the nose of a healthy, 53-yearold non-smoking Caucasian male (73 kg mass, $173 \mathrm{~cm}$ height). Swift's replica was assembled from a number of 3-mm thick plastic coronal elements e carved by hand with a high-speed cutting tool whose diameter or size was unspecified. The outlines of the airway on the plastic elements were traced on each side of the junction of two elements and the elements were cut to follow the transition from one image to the next.

Schreck et al. (1993) constructed a physical nasal model by processing the internal anatomy of the airways obtained from magnetic resonance images (MRI) of the head and neck of one healthy adult subject. Twenty-seven Plexiglas (clear plastic) plates with a $12 \mathrm{~mm}$ thickness were cut using three times enlarged digitized coronal planes (spaced in $4 \mathrm{~mm}$ increments before enlargement) of the nasal airways. After cutting and polishing, the plates were assembled together to form a three times scaled single nasal cavity model of the left passage, which can be seen in Figure 2.2.

Guilmette and Gagliano (1994) described a method to created 3D physical human nasal cavity models from MRI data. The subject of their study was a 59-year-old healthy male non-smoker. Two models were generated, one containing the region from the tip of 
the nostril to the posterior pharynx, and one excluding the olfactory region. Three dimensional lofted surfaces which described the nasal airways were created using CAD software and airway perimeter coordinates were obtained from the MRI scans. Then seventy $1.5-\mathrm{mm}$-thick black acrylic plates containing the airways were cut out using a computer-controlled micromilling machine using a $0.5 \mathrm{~mm}$ end. This manufacturing method had the advantages of accuracy and reproducibility compared with Swift's method. Zwartz and Guilmette (1999) modified the model created by Guilmette and Gagliano (1994) to generate a third model, by replacing the segments containing the olfactory region. Zwartz and Guilmette (2001) also use this method to construct two nasal cavity replicas based on the same MRI scans used by Swift (1991). One of the replicas did not contain the entire olfactory region and the other one included a $9 \%$ larger olfactory volume.

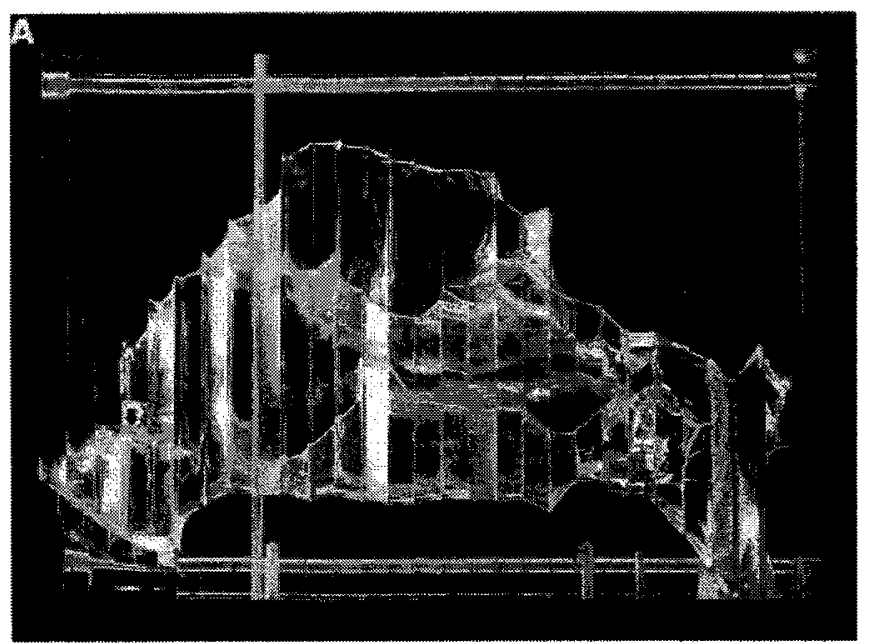

Figure 2.2 Side view of physical nasal cavity model created by Schreck et al., (1993)

Hopkins et al. (2000) established a procedure for constructing a transparent rectangular box containing a model of the nasal cavity for PIV measurement by combining the rapid prototyping (RP) and the curing of clear silicone. Kim and Son 
(2003) improved the procedure for producing a better cavity model with high-resolution computerized tomography (CT) scan data and surface rendering and extended this work to deal with airflow in an abnormal nasal cavity with adenoid vegetation and asymmetry.

Among a large number of available rapid prototyping technologies in the marketplace stereolithography is widely used to build nasal cavity replicas. The advantages of Stereolithography method are that it is convenient to produce physical replicas of complex geometries and it has improved accuracy compared to other means such as hand carving.

Different manufacturing methods have different effects on the nasal replica, such as the surface roughness. As a result, the measured results (velocity or particle deposition fraction) using replicas manufactured by different methods would be influenced (Kelly et al., 2004). Kelly et al. (2004) created two physical models from the same MRI scans of Swift (1991) using stereolithography (SL), and then measured the inertial paretic deposition fraction of these two replicas. One of the models was manufactured by Accelerated Technologies, Inc. (ATI, Austin, TX, USA) using SLA 7000 (3D System, INC., Valencia, CA, USA), and thus was referred as the SLA replica. The other one was referred as Viper replica, which was manufactured using a Viper Si2 machine (3D System, INC.). Kelly et al. (2004) found that their data showed lower deposition fraction when compared with other deposition results of previous researchers in nasal replicas created from the same set of MRI scans. Conclusion was drawn that certain part of the differences in deposition fraction can be attributed to differences in surface roughness resulted from different methods used to manufactured the replicas. 
In summary, modern imaging technology such as $\mathrm{CT}$, magnetic resonance imaging MRI along with the unprecedented development of CAD solid modeling technology make it possible to gather detailed anatomic information of the human nasal cavity and create 3D human nasal cavity models. Advanced manufacturing technology including Rapid Prototyping (i.e., Stereolithography etc.) offer several ways to build physical replicas of human nasal cavity models from 3D models stored in an electronic format, and thus facilitate investigate particle deposition and airflow patterns in human nasal cavity experimentally and numerically.

For this present application, the replica of standardized human nasal cavity model was produced using Soluble Support Technology 1200es Dimension 3D Printer (Stratasys, Inc., Eden Prairie, USA). The minimum layer thickness of the Dimension 3D printer was $0.25 \mathrm{~mm}(0.010$ inches $)$. The details about the generation of the standardized human nasal cavity model and the construction of the physical replica of it will be provided in Chapter 3 and Chapter 4.

\subsection{Experimental studies of airflow patterns and particle deposition in human nasal cavities}

\subsubsection{Experimental studies of nasal airflow}

Detailed knowledge of airflow patterns in the human nasal cavity is the foundation of understanding the physiological and pathological aspects of nasal breathing and patterns 
of particle transportation and deposition in the nasal cavity. Several studies (e.g. Swift and Proctor, 1977; Girardin et al., 1983; Hahn et al., 1993; Schreck et al., 1993; Park et al., 1997; Kelly et al., 2000; Hopkins et al., 2000; Doorly et al. 2008) have utilized physical models of the nasal cavity in an effort to understand the relationship between nasal anatomy and the distribution of inhaled and exhaled airflow.

The highly complex geometry and compact size of nasal cavity makes the investigation of nasal airflow patterns difficult and challenging. There are two ways to solve the problem of measurement of airflow field in the human nasal cavity (as well as in the oral cavity and trachea). The first approach is to use an $\mathrm{N}$ times $(\mathrm{N} \geq 2)$ scaled nasal cavity model (Hahn et al., 1993; Schreck et al., 1993; Park et al., 1997; Kelly et al., 2000). The second approach is to build a transparent colourless model with an indexmatched fluid (usually is optimal glycerol /water) and use standard PIV imaging and laser delivery system, as reported by Hopkins et al. (2000) and Doorly et al. (2008). These two approaches can be adopted together or separately to facilitate the measurement of complex flow field in human nasal cavity.

Adoption of these two approaches raises an important issue of similitude, i.e., the measurements must satisfy following criteria: Geometric similarity, Kinematic similarity and Dynamic similarity (see Kline, 1986 for more details about the similitude in the engineering measurements). The values of geometric dimensions, velocity of fluid, flow rates, pressure losses and time scales obtained using scaled models must be rescaled to real quantities.

Reynolds number matching is usually used to solve the problem (Hahn et al., 1993; Schreck et al., 1993; Park et al., 1997; Kelly et al., 2000), which means the Reynolds 
Number in model must be equal to those of real air breathing condition to assure the dynamic similarity.

$$
\operatorname{Re}=\frac{U L}{v}=\text { constant }
$$

Thus, the relation between measured and real values can be obtained using:

$$
\begin{gathered}
\frac{U_{\text {real }}}{U_{\text {measured }}}=\frac{L_{\text {model }}}{L_{\text {real }}} \frac{v_{\text {real }}}{V_{\text {model }}}=\frac{\xi}{N} \\
\frac{\dot{Q}_{\text {real }}}{\dot{Q}_{\text {measured }}}=\frac{U_{\text {real }}}{U_{\text {measured }}} \frac{L_{\text {real }}^{2}}{L_{\text {model }}^{2}}=\frac{\xi}{N} \frac{1}{N^{2}}=\frac{\xi}{N^{3}}
\end{gathered}
$$

in which, $U$ is velocity, $\dot{Q}$ is the flowrate, the $N$ is the times of the scaled model (also called length scale $\left.L_{\text {model }} / L_{\text {real }}\right), \xi$ is the viscosity ratio $\left(v_{\text {model }} / \nu_{\text {real }}\right)$.

If not stated otherwise, the values of the measurements (such as velocities, flowrates etc.) of previous researchers are all real values (physiological values) rather than model quantities (measured values).

Swift and Proctor (1977) performed the first quantitative in vitro velocity measurements. They measured the velocity of the air in the fluid field by inserting a miniature Pitot device into the flow passage of a nasal cavity model, which was made from a nasal cast of a cadaver. The flow was reported to be laminar at a flow rate of $125 \mathrm{~mL} / \mathrm{s}$. Turbulent flow was detected downstream of the nasal valve at a flow rate of $208 \mathrm{~mL} / \mathrm{s}$.

Kelly et al. (2000) noted several limitations for experimental study of airflow patterns in the nasal cavity using Pitot tubes with models from cadavers. These included issues associated with the approximate geometry due to both the flat septum and 
variations between cadavers and live subjects that result from post-mortem shrinkage of tissue, the invasive nature of the Pitot device, which may deflect the air stream, resulting in a biased total pressure reading, and the small size of the cast model.

In order to solve the problem related to the small size of the cast model, Hahn et al. (1993) constructed a 20 times replica of a nasal cavity from computerized axial tomography (CAT) scans spaced in $2 \mathrm{~mm}$ increments from the nostril of the nose to the beginning of the nasopharynx. Pointwise velocity measurements, made in five planes throughout the model with a hotfilm anemometer, indicated that the flow was laminar at flow rates up to around $200 \mathrm{~mL} / \mathrm{s}$ (e.g. $12 \mathrm{~L} / \mathrm{min}$ ). It was found that $\sim 50 \%$ of inhaled air flows through the combined middle and inferior airways, and $\sim 14 \%$ flows through the olfactory region at all flow rates studied.

Additionally, experimental studies on human nasal airflow patterns have been conducted using laser Doppler velocimetry (LDV) (Girardin et al., 1983). There are several advantages in using LDV to study human nasal airflow patterns, among which the most important one is that it allows the statistical averages of mean velocity and turbulence intensity to be determined. Due to the highly complex airflow patterns in the nasal cavity, as well as its three-dimensionality, high spatial gradients, and turbulence, the measurements of airflow patterns using point measurement techniques such as hotwire anemometry and LDV are difficult, expensive and impractical. These measurement techniques require that the particle density be sufficiently large for a reasonable data rate, and they require thousands of individual measurements to resolve the flow (Heenan et al., 2003). 
In contrast, particle image velocimetry (PIV) is a powerful technique to measure fluid velocities, and is able to determine the instantaneous flow field over two- or threedimensional domains. Park et al. (1997) were the first to use a two-dimensional measurement technique, digital particle image velocimetry (DPIV), to study nasal airflow patterns. A 33 times scaled nasal replica was generated from approximately nine coronal slices of anatomic data (from nostril to nasopharynx). Their results showed a noteworthy portion of the high-velocity flow occurred in the olfactory region. Their work was limited because the resolution of anatomic data used for their model was not high enough, and because vector plots in five sub-locations of each plane, forming the global velocity field, merged into others.

Kelly et al. (2000) used particle image velocimetry to determine two-dimensional instantaneous velocity vector fields in parallel planes throughout a two-times scaled model of a right human nasal cavity, and used a steady flow rate of $125 \mathrm{~mL} / \mathrm{s}$ for inhaled air. The model adopted in their research was fabricated from 26 CT scans, using rapid prototyping techniques. The vector plots of measured results show that the flow was laminar and the highest velocities were detected at the nasal valve and along the floor of the nasal cavity in the inferior airway. The nasal valve has the smallest cross-sectional area in the nasal cavity, and thus there is high-velocity flow in that region. The relatively low flow in the olfactory region appears to protect the olfactory bulb from particulate pollutants. Low flows were also observed in the nasal meatuses. Comparison of sequentially recorded data suggests a steady flow.

Hopkins et al. (2000) performed PIV measurement using a nasal passage model made of clear silicone. Their measurements showed that an eddy can be seen in the 
region just posterior to the nasal valve. They conjectured that this flow structure was a result of the adverse pressure gradient that is caused by the abrupt expansion in crosssectional area in this region.

\subsubsection{Experimental study of particle deposition in the airway passages}

The deposition occurring within the human upper respiratory system has been a primary topic of interest for decades (Prattle, 1961; Johnston and Schroter, 1979; Chan et al., 1980; Kim and Iglesias, 1989; and Cohen and Asgharian, 1990, Lennon et al., 1998). The nasal cavity region has received particular attention (e.g. Heyder and Rudolf, 1977; Itoh et al., 1985; Heyder et al., 1986; and Cheng et al., 1988, Etherington et al., 1998) because of its highly complex geometry and important function in nasal drug delivery. Most information about particle deposition in the various human airway passages has been obtained through experimental work. In general, experimental measurements of deposition fraction in anatomical airways can either be conducted in vitro (using replicas) or in vivo (measurements directly on live patients).

\subsubsection{In-vivo experimental studies of nasal particle deposition fraction}

In typical in vivo measurements of nasal particle deposition, the aerosol concentration is measured before it is inhaled into the nasal cavity and after it is exhaled through the mouth (Pattle 1961; Hounam et al., 1969; Heyder and Rudolf, 1977; Stahlhofen et al., 1989; Cheng et al., 1996; Swift and Strong, 1996; Kesavanathan and Swift, 1998). As 
shown in Figure 2.3, Pattle (1961) performed in vivo particle deposition experiments on a human subject, and results showed that the human nasal cavity particle deposition fraction can be represented as a unique function of the impaction parameter, $d_{a}^{2} Q$. The data can be fitted with the following equation:

$$
D F=1-\exp \left(-a^{\prime} d_{a}^{2} Q\right)
$$

where $d_{a}$ is the aerodynamic diameter, $Q$ is the air flow rate in $\mathrm{L} / \mathrm{min}$, and $a^{\prime}=0.00241 \pm 0.002($ mean $\pm S E M)$ with $r^{2}=0.94$, indicating a good fit.

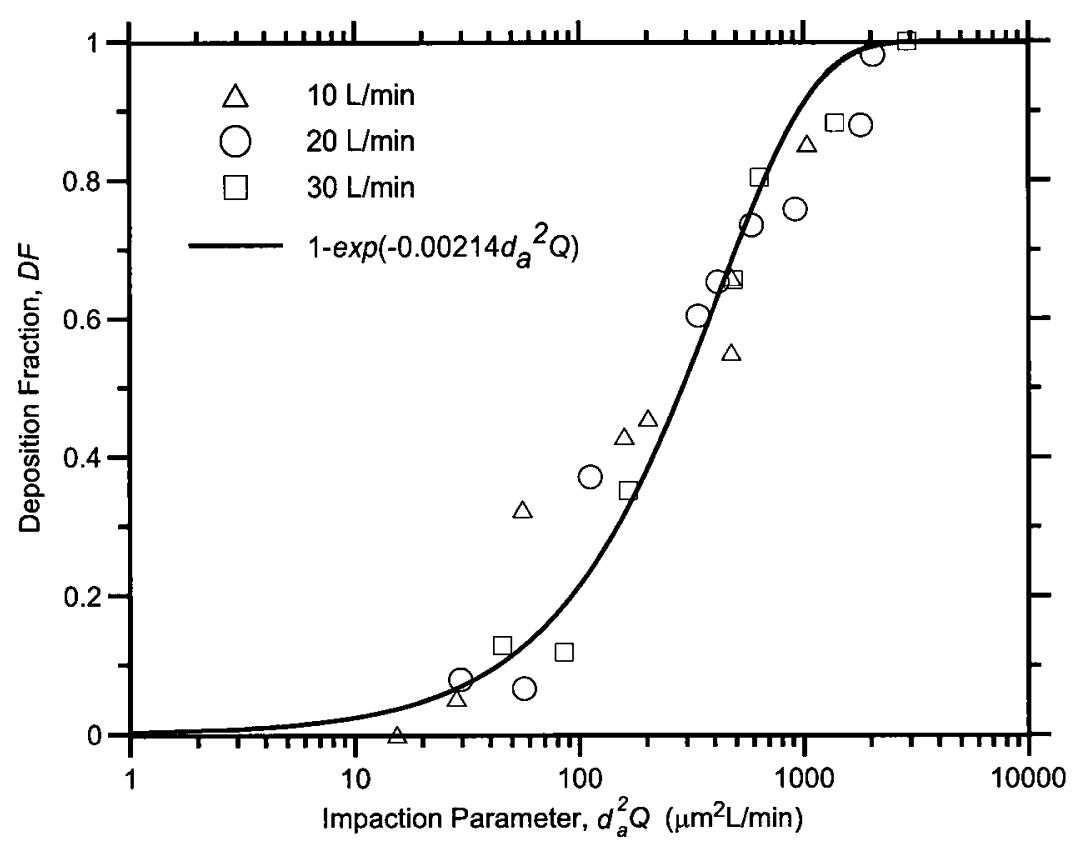

Figure 2.3 Deposition fraction in human nasal cavity illustrated as a function of impaction parameter for an adult human volunteer (Pattle, 1961)

Similarly, inspiratory nasal deposition data for three subjects in a study by Hounam et al. (1969) and mean values of deposition measurements of six subjects by Landahl and Black (1947) could also be fitted by the same expression. Heyder and Rudolf (1977) measured both inspiratory and expiratory nasal deposition data in four adult male 
subjects. All data from the four subjects showed that the inspiratory and expiratory nasal depositions were not statistically different (data not shown). This analysis indicates that the assumption of equal nasal deposition fraction for the inspiratory and expiratory flows used in most lung deposition models is reasonable.

Cheng (2003) summarized and analyzed available in vivo data in human volunteers of Landahl and Black (1947), Pattle (1961), Hounam et al. (1969), Giacomelli-Maltoni et al. (1972), and Heyder and Rudolf (1977). Cheng assumed that the airway dimensions cause the variability in the nasal deposition values, and he proposed Equation 2.5 as the correct mathematical expression for impaction deposition:

$$
D F=1-\exp (-110 S t k)
$$

where $S t k=\rho^{0.5} d_{a}^{2} Q / 18 \rho A_{\min }^{1.5}$, is the dimensionless Stokes number. The corresponding value of the minimum cross-sectional area in the nasal airway for each subject can be calculated using Equation 2.6:

$$
A_{\min }=\left(\frac{0.00982}{\alpha^{\prime}}\right)^{2 / 3}
$$

Values of $\mathrm{A}_{\min }$ for the eight subjects (one by Landahl and Black, 1947; four by Heyder and Rudolf ,1977; three by Hounam et al. 1971) ranged from $1.11-3.90 \mathrm{~cm}^{2}$. The value of $\mathrm{A}_{\min }$ corresponding to the mean value of $\alpha^{\prime}=0.00390$ was $2.11 \mathrm{~cm}^{2}$.

Cheng et al. (1996) reported measurements of the nasal airway dimensions for 10 adult male volunteers using MRI scans. The value of $A_{\min }$ ranged from $1.28-3.10 \mathrm{~cm}^{2}$ with a mean value of $2.08 \mathrm{~cm}^{2}$. The calculated values of $A_{\min }$ from this analysis of deposition data are in approximately the same range, indicating that Cheng et al.'s (1996) values were reasonable. 
As illustrated in Figure 2.4, Cheng (2003) summarized previous in vivo nasal deposition data using the same mathematical expression originally proposed by Pattle (1961) $D F=1-\exp \left(-a^{\prime} d_{a}^{2} Q\right)$. The centre curve is for a mean value of $0.00309\left(\mathrm{~A}_{\min }=\right.$ $\left.2.16 \mathrm{~cm}^{2}\right)$ and the curves for the upper and lower bounds correspond to $0.00839\left(\mathrm{~A}_{\min }\right.$ $\left.=1.11 \mathrm{~cm}^{2}\right)$ and $0.00128\left(A_{\min }=3.90 \mathrm{~cm}^{2}\right)$, respectively. The three curves summarized by Cheng (2003) represent an estimate under experimental conditions and can be used as a comparison standard for nasal cavity deposition.

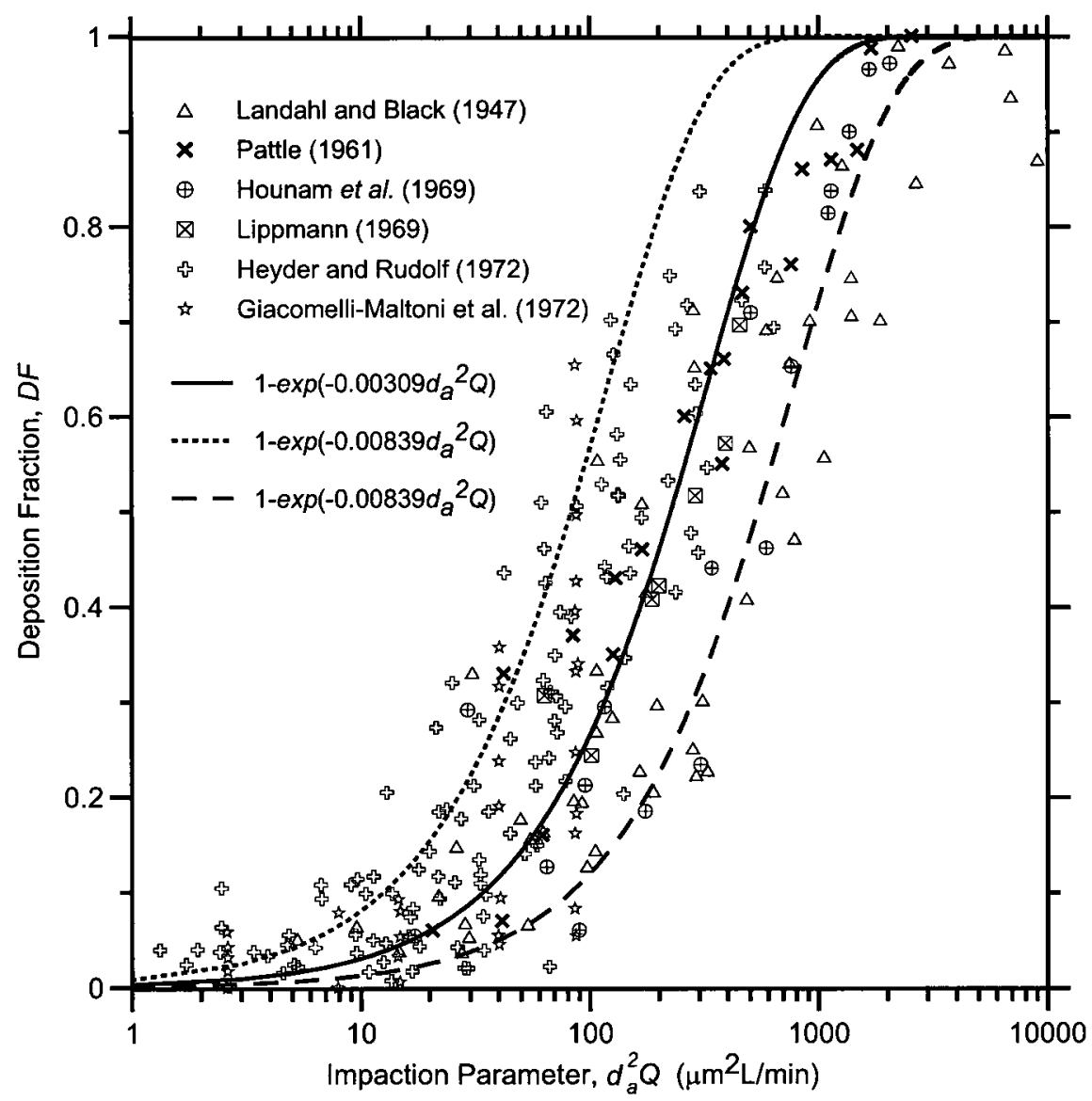

Figure 2.4 Summary of nasal deposition fraction as a function of impaction parameter in human volunteers (after Cheng, 2003)

In recent nasal particle deposition research, in vivo studies using scintigraphy (radiolabeled aerosol + gamma camera) have been widely adopted, and several authors 
have performed qualitative studies of in vivo nasal deposition using radiolabelled particles (see Suman et al., 1999; Giroux, 2005). Figure 2.5 shows the in vivo controlled dispersion particle deposition acquired using scintigraphy by Giroux in 2005, which provided visual particle distribution patterns using passive-diffusion nebulizers and nasal spray pumps. However, Giroux (2005) did not provided any quantitative analysis of total or regional particle deposition fraction.
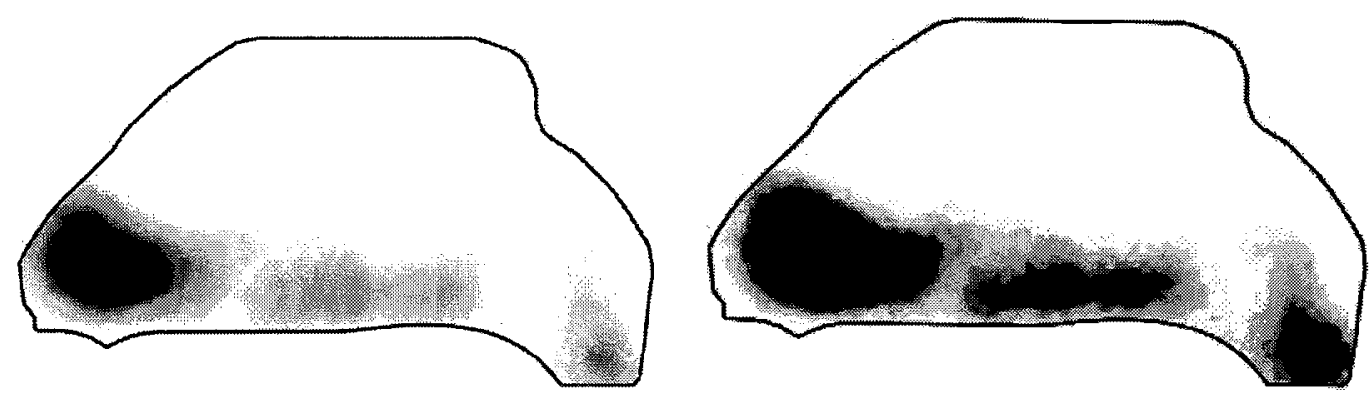

Figure 2.5 Particle deposition in the nasal cavity using an in vivo scintigraphy method (left: using passive nebulizer; right: using nasal spray pump (Giroux, 2005)

\subsubsection{In vitro experimental studies on nasal particle deposition fraction}

In vivo studies on humans are limited by health and ethical issues since relatively large amounts of radioactivity are required to measure deposition in different nasal regions. To avoid high-dose radiation exposure, as well as to obtain more reproducible results, in vitro experiments, using artificial nasal cavities (nasal casts or replicas of nasal airways), have been used to complement difficult and expensive in vivo studies for decades. Apart from safety, there are several advantages of measuring particle deposition fraction using a human nasal cavity model (Zwartz and Guilmette, 2001). First, advanced manufacturing technology makes it possible to build an accurate nasal airway model with features such as a vestibule, nasal valve, olfactory region, turbinates and nasopharynx regions. In 
addition, all parameters (i.e. inhalation flow rate, particle size, particle type), which may influence the nasal particle deposition, can be controlled and measured independently and systematically. This makes it easy and convenient to determine how these parameters affect the total and regional particle deposition fraction.

In in vitro experiments, particles were usually drawn into the model using a vacuum pump that produces a constant flow rather than cyclic flow patterns or realistic human breathing. Several in vitro measurements have been published including those by Swift (1991), Gradon and Podgorski (1992), Lennon et al. (1998), Cheng et al. (1998), Zwartz and Guilmette (2001), Cheng et al. (2001), Janssens et al. (1998), Kelly et al. (2004), and Garcia et al. (2009), as summarized in Figure 2.6 together with the semi-empirical fit line of in-vivo data summarized by Cheng (2003).

Except for the data from Swift (1991), which were obtained using a hand-carved replica, all of the previous in vitro data follow a similar trend, which falls below the summary in vivo curve of Cheng (2003). The difference between in vitro and in vivo measurements is not unexpected because the principles of the two measuring methods are different (a more detailed discussion of this will be presented in Chapter 5 with present in vitro measured results in the standardized human nasal cavity model).

In Figure 2.6, all of the data shown in blue were obtained by a variety of researchers using geometries manufactured from the same set of MRI scans for a single human subject originally reported by Swift (1991). These include the SLA and Viper models of Kelly et al. (2004), the first model of Garcia et al. (2009), and the models of Zwartz and Guilmette (2001), Cheng et al. (2001), and Swift (1991). By comparing the results of different nasal models built from MRI scans of the same individual, but with different 
manufacturing methods (summarized in Section 2.3.1), it can be seen that that deposition is larger when surface roughness is larger. Garcia et al. (2004) investigated the effect of nasal cavity geometry on particle deposition fraction. Their results showed that for the same impaction parameter, the wider nasal passages (subject 1 and subject 3 , male) had higher particle deposition efficiencies (see Figure 2.6), which indicated that nasal deposition increases as the characteristic diameter of the nasal passages increases..

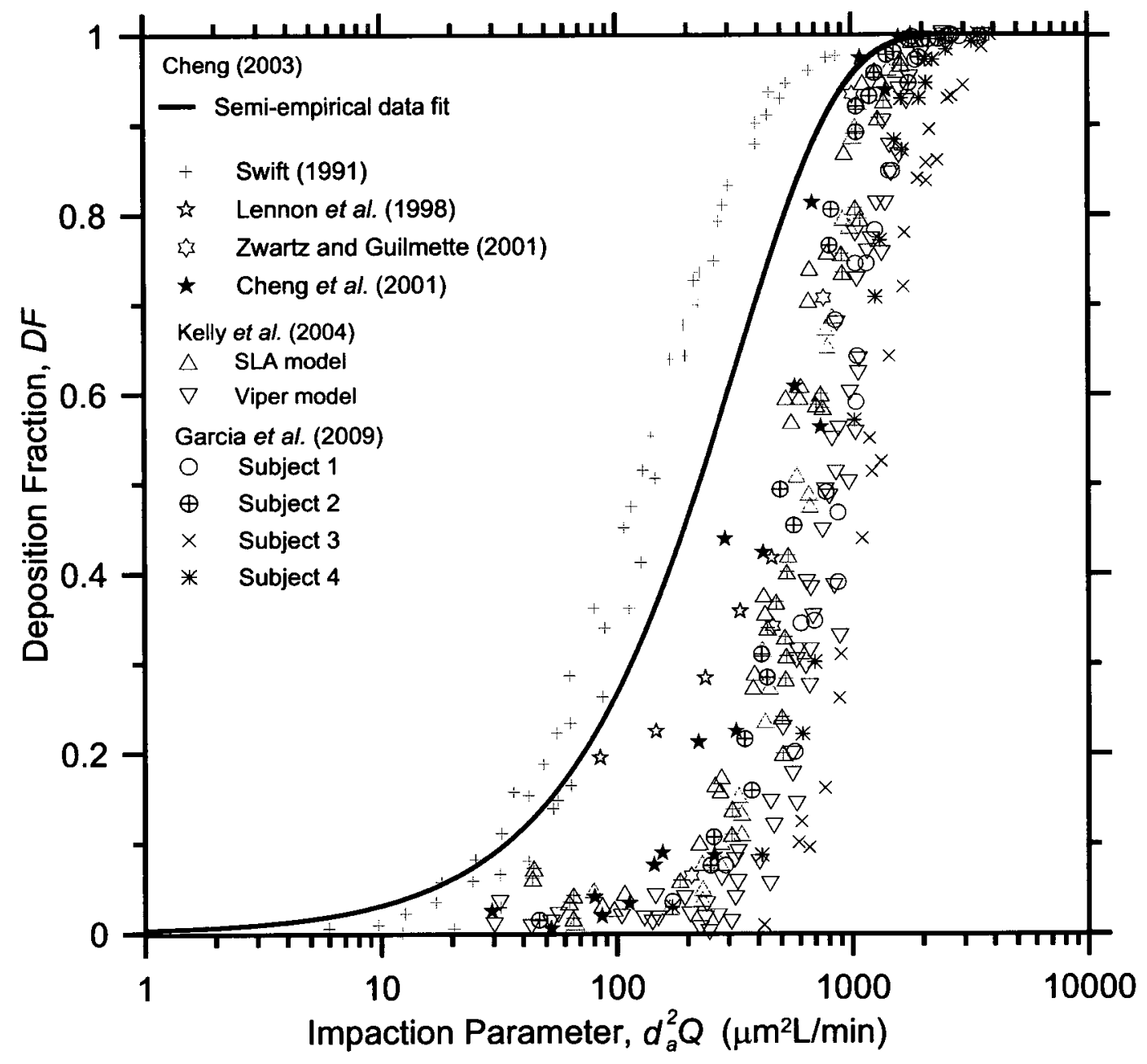

Figure 2.6 Summary of in vitro measurements of nasal deposition fraction

To better understand the patterns of particle deposition, Häußermann et al. (2002) measured the differences in total deposition in the nose arising from differences in the 
breathing patterns. The cyclic human breathing patterns in their study were produced by the breathing simulator (Häußermann et al., 2002) based on the data of breathing patterns of a healthy Caucasian male volunteer. Monodisperse particles (diameters varied from 1.7 to $10 \mu \mathrm{m}$ ) were studied in a cast of nasal cavity using different human breathing patterns (maximum flow rates $=10,20,30,40$ and $50 \mathrm{~L} / \mathrm{min}$ ) as well as under constant flow rate conditions.

Their results for small particles (between 1.7 and $3 \mu \mathrm{m}$ ) showed a much higher deposition using constant flow rates than deposition caused by cyclic human breathing with the same average flow rate. This difference decreased for higher values of the impaction parameter (i.e., when the particles became larger or flow rate increased). It was conjectured by the authors that the reason could be the different flow conditions in the nasal cavity model caused by different breathing patterns, since it is more likely for cyclic breathing patterns to cause vortices at different locations in nasal cavity than constant flow.

However, it should be noted that Häußermann et al. (2002) acknowledged that their results showed the opposite trend compared with the results of similar particle deposition experiments performed by other researchers using lung cast (Gurman et al., 1984). In addition, steady flow might be more realistic than cyclic flow in the drug delivery. In present numerical and experimental investigation of particle deposition in the standardized human nasal cavity, the target application is medical drug delivery. Thus, steady flow rates were used instead of cyclic flow. 


\subsection{Numerical simulation}

Numerical simulation using computational fluid dynamics (CFD) is also a very useful, and widely adopted tool for studying nasal aerosol deposition, which is in general much easier to implement than experiments. However, CFD has its own disadvantages and limitations, one of these limitations being the reliability of the obtained results. The reliability of results is dependent on factors such as the numerical algorithms of the equations, the mathematical formulation used to process the simulation, the boundary conditions, etc. Furthermore, where turbulent flow is concerned, the chosen turbulence model is important; it is difficult to choose because there is no single turbulence model that can reliably predict all turbulent flow with sufficient accuracy.

\subsubsection{Overview of simulation of particles in turbulent flow}

To model numerically the particle-fluid two-phase flow, it is important to clarify the coupling between the particle motion and its surrounding fluid and thus determine the appropriate numerical method. Elgobashi (1994) presented an overview of the simulation

of particles in turbulent flows and proposed a map of interactions between particles and fluid turbulence. This map of interactions is shown in Figure 2.7. 


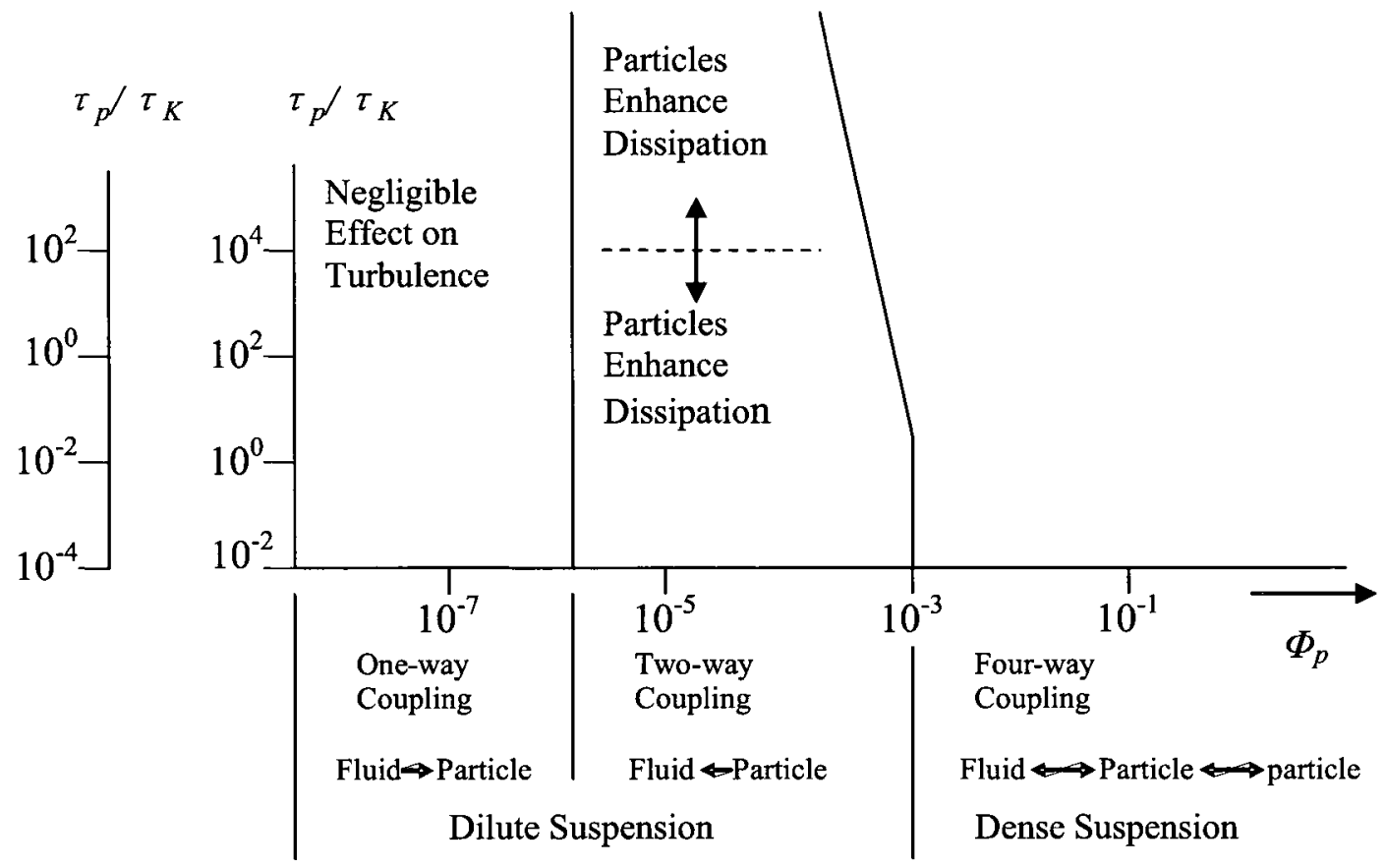

Figure 2.7 Interactions between particles and fluid turbulence

The quantities in the dimensionless coordinates shown in Figure 2.7 are defined below.

$\Phi_{p}:$ volume fraction of particles, $\Phi_{p}=M V_{p} / V$

$M:$ number of particles

$V_{p}:$ volume of a single particle

$V:$ volume occupied by particles and fluid

$d:$ diameter of particle

$\tau_{p}:$ particle response time, $\tau_{p}=\rho_{p} d^{2} /\left(18 \rho_{f} v\right)$ for Stokes flow

$\tau_{K}:$ Kolmogorov time scale, $\tau_{K}=(v / \varepsilon)^{1 / 2}$

$\tau_{e}: \quad$ turn-over time of a large eddy, $\tau_{e}=l / u$

$v: \quad$ kinematic viscosity of the fluid 
$\rho: \quad$ material density and the subscripts $p$ and $f$ denote respectively the particle and carrier fluid

$l$ : length scale of the energy containing eddies,

$u$ : $\quad$ RMS fluid velocity

$\varepsilon: \quad$ the dissipation rate of turbulence kinetic energy.

For very low values of dispersed-phase volume fraction $\left(\Phi_{p}<10^{-6}\right)$, the particles have negligible effects on turbulence, and the interaction between the particles and turbulence referred to as "one-way coupling". This means that in this regime, particle dispersion, depends on the state of turbulence, but due to the negligible concentration of the particles, the momentum transfer from the particles to the turbulent flow has an insignificant effect on the flow. In the second regime, $10^{-6}<\Phi_{p}<10^{-3}$, the momentum transfer from the particles is large enough to alter the structure of the turbulence. This interaction is called "two-way coupling". In this regime, and for a given value of $\Phi_{p}$, lowering the ratio of the particle response time $\tau_{p}$ (e.g. smaller particle diameter for the same particle material and fluid viscosity) increases the surface area of the particulate phase, hence increasing the dissipation rate of turbulence energy. Moreover, as $\tau_{p}$ increases for the same $\Phi_{\mathrm{p}}$, the particle Reynolds number, $\mathrm{Re}_{\mathrm{p}}$, increases and at values of $\operatorname{Re}_{\mathrm{p}} \geq 400$, vortex shedding takes place resulting in enhanced production of turbulence energy. This means that the existence of particles can augment the turbulence if the ratio of the particle response time to the turnover time of a large eddy is greater than unity, or can attenuate turbulence if the ratio is less than unity. The vertical coordinate $\left(\tau_{\mathrm{p}} / \tau_{\mathrm{e}}\right)$ is related to the other coordinate $\left(\tau_{\mathrm{p}} / \tau_{\mathrm{k}}\right)$ by the turbulence Reynolds number $\left(\operatorname{Re}_{\lambda}=\mathrm{ul} / v\right)$ 
since $\left(\tau_{\mathrm{p}} / \tau\right)=\operatorname{Re}_{\lambda}^{1 / 2}$. Flows in the first two regimes discussed above are often referred to as dilute cases. In the third regime, $\Phi_{p}>10^{-3}$, flows are referred to as dense cases. Here, in addition to two-way coupling between particles and turbulence, particle/particle collisions take place, and this regime is called four-way coupling. As $\Phi_{\mathrm{p}}$ increases and approaches 1, a granular flow in which there is no fluid is obtained.. The current research is concerned with dilute flows only in which one-way coupling can be assumed.

Interactions between turbulence and particles involve: 1) turbulent dispersion of the particles (the effects of turbulence on the particles), and 2) modification of the carrierphase turbulence structure (the effects of the presence of particles on the turbulence). Since one-way coupling will be assumed in present work, only turbulent dispersion of the particles will be taken into account in the present simulation. Particle dispersion in turbulence is often simulated using the stochastic (Monte Carlo) method, in which the turbulent field is represented by a random number generator. Conventional stochastic Lagrangian computations are also called stochastic discrete-delta function models (Chen and Pereira, 1998).

\subsubsection{Modeling methodology}

Prediction of particle deposition is usually handled in one of two approaches (Mashayek and Pandya, 2003). The first approach is Eulerian, where particles are assumed to move toward the adjacent wall by turbulent diffusion and then reach the wall by a free flight mechanism (see Friedlander and Johnstone,1951; Ganic and Mastanaiah, 1981; Jackson et al., 1997). The second approach is Lagrangian, which is also called "trajectory model" 
where trajectories of individual particles are calculated by solving the particle equation of motion. In the most commonly used Lagrangian approach, the single-phase flow is calculated by using RANS (Reynolds averaged Navier-Stokes) equations (for example, the $k-\varepsilon, k-\omega$, and shear stress transport, SST, turbulence model equations), LES (large eddy simulation), or DNS (direct numerical simulation), then individual particles are numerically released in the computational domain by following the particle equation of motion. The Lagrangian approach has a computational expense that is higher than the Eulerian approach (Elgobashi 1994), but it is able to provide a more realistic and flexible model of particle deposition than the Eulerian one. As noted above, when the particle concentration is very low, the primary flow is assumed to be unmodified by the presence of particles (i.e., "one-way coupling"); this assumption significantly facilitates simulations of particle motion. On the other hand, when the particle concentration is not very low, the mutual interaction between the primary flow and the particles must be incorporated into the simulation (i.e., "two-way coupling").

The most sophisticated and accurate approach for modeling particle deposition in turbulent boundary layers is DNS. In DNS the Navier-Stokes equations are solved without modeling and the main characteristics of the flows can be correctly predicted by DNS. DNS has been used successfully in a number of studies that have increased our fundamental understanding of many of the mechanisms governing deposition (Mclaughlin, 1989; Brooke et al., 1994). However, DNS is extremely expensive and requires large computational time, as the grid density required is proportional to $\operatorname{Re}^{3}$ (Luo et al., 2004). Thus, for high Reynolds numbers and complicated geometries, such as human airway geometry, the mesh sizes required for DNS are prohibitive. 
LES is an approach which is not as severely restricted in the range of Reynolds numbers as DNS. In LES, the large, energy-containing scales of motion are calculated directly, while only the effect of the smallest (subgrid) scales of motion are modeled. This approach works well since particle dispersion is determined mainly by the large scales. Thus, LES is being used increasingly in calculations of turbulent flows (Akselvoll and Moin, 1993; Squires and Piomelli, 1995) and prediction of particle deposition in turbulent flows (Wang and Squires, 1996; Yuu et al., 2001; Pandya and Mashayek, 2002).

The Eddy-particle interaction model is a Lagrangian approach used to track particles in the simulation of flows laden with particles. Given initial conditions of velocity and position, the motion of a single particle is calculated by treating it as a series of interactions with discrete eddies having a characteristic size (eddy length), lifetime and velocity. Graham and James (1996) classified eddy interaction models, which have appeared in the literature, into the following three types:

(1) The characteristic eddy scales remain constant throughout the flow (Hutchinson et al., 1971; James et al., 1980);

(2) The characteristic eddy scales are obtained from a numerical simulation of the turbulent primary flow (Gosman and Ioannides, 1981; Govan et al., 1989; Kallio and Reeks ,1989);

(3) Alternative models, in which, besides representative length scale, lifetime and velocity scale, other forms of Langragian or Eulerian velocity auto-correlation functions were required (Berlemont el al., 1990; Burry and Bergeles, 1990) 
From a practical point of view, the use of the Reynolds-averaged Navier-Stokes (RANS) equations, such as the $k-\varepsilon$ and $k-\omega$ turbulence model equations, is a costeffective and preferable way to predict the primary flow. Though the RANS equations provide only one-point turbulence statistics, it is of great significance to demonstrate that once correct turbulence statistics are given, the one-way coupling Lagrangian Eddyparticle interaction model (EIM) can give adequate prediction of particle deposition on the walls. It is foreseen that a combined use of RANS/EIM equations with newly emerging experimental techniques will provide a way of predicting accurate near-wall turbulence statistics of the primary flow and particle deposition in the nasal cavity through careful optimization of parameters in the RANS equations.

Both DNS and LES require no model for the calculation of the particle trajectories, since they provide the filtered and the actual instantaneous values of fluid velocities, but they are computationally much more expensive than the RANS/EIM approach. Both experimental (aerosol deposition) and numerical methods (RANS/EIM, LES) will be explored in the present work to simulate the airflow and deposition patterns in a realistic and idealized nasal cavity.

\subsubsection{Previous Simulations of particles in human respiratory tract}

CFD simulations can be used in the prediction of detailed total and regional deposition patterns within the human respiratory tract, which are currently difficult to determine from in vivo or in vitro experiments. Much work has been done to investigate particle deposition in the human respiratory tract using CFD, e.g., DeHaan (2001) and Matida et al. (2003) in the oropharynx (mouth-throat), Zhang et al. (2003) using oral airways (i.e., 
mouth to trachea), Tang et al. (2004), Inthavong et al. (2006), Liu et al. (2007), Tian et al. (2007), Shi et al. (2007), and Shanley et al. (2008) using the nasal tract (summarized in Table 2.3), Yu et al. (1998) for particle diffusion in human upper respiratory system and Sarangapani and Wexler (2000) using an extrathoracic model (i.e., nasal to larynx).

Table 2.3 Summary of previous numerical simulations of particle deposition in human nasal cavity

\begin{tabular}{|c|c|c|c|c|}
\hline $\begin{array}{l}\text { Researcher } \\
\text { and year }\end{array}$ & $\begin{array}{c}\text { Data source of } \\
\text { geometry of nasal cavity }\end{array}$ & $\begin{array}{c}\text { Simulation environment and } \\
\text { method }\end{array}$ & $\begin{array}{l}\text { Flow rates, particle } \\
\text { sizes and density }\end{array}$ & $\begin{array}{c}\text { Other } \\
\text { information }\end{array}$ \\
\hline $\begin{array}{l}\text { Inthanvong } \\
\text { et al., } 2006\end{array}$ & $\begin{array}{l}\text { CT scans of a 25-year-old } \\
\text { healthy, non-smoking } \\
\text { Asian male }(73 \mathrm{~kg}, 173 \\
\mathrm{cm})\end{array}$ & $\begin{array}{c}\text { FLUENT6.1.2; realizable k- } \varepsilon \\
\text { model for airflow simulation, hybrid } \\
\text { Lagrangian particle tracking method } \\
\text { (Discrete Random Walk model and } \\
\text { mean flow tracking) }\end{array}$ & $\begin{array}{c}20,30 \text { and } 40 \mathrm{~L} / \mathrm{min} ; \\
1 \sim 30 \mu \mathrm{m} \\
1000 \mathrm{Kg} / \mathrm{m}^{3}\end{array}$ & $\begin{array}{l}\text { Smooth wall; } \\
\text { particles } \\
\text { deposit when } \\
\text { touch the wall }\end{array}$ \\
\hline $\begin{array}{l}\text { Liu et al., } \\
2007\end{array}$ & $\begin{array}{l}\text { CT scans of a healthy } 25- \\
\text { year-old man (Kelly et al, } \\
2000 \text { ); improve the nostril }\end{array}$ & $\begin{array}{l}\text { CFX 5.1; SST (Shear Stress } \\
\text { Transport) k- } \omega \text { based Model and } \\
\text { LES for airflow simulation; Eddy } \\
\text { Interaction Model (turbulent tracking } \\
\text { and mean flow tracking }\end{array}$ & $\begin{array}{c}7.5,15,30,45 \text { and } \\
60 \mathrm{~L} / \mathrm{min} ; \\
0.35 \sim 16 \mu \mathrm{m} \\
913 \mathrm{Kg} / \mathrm{m}^{3}\end{array}$ & $\begin{array}{l}\text { Smooth wall; } \\
\text { particles } \\
\text { deposit when } \\
\text { touch the wall }\end{array}$ \\
\hline $\begin{array}{l}\text { Tian et al., } \\
2007\end{array}$ & $\begin{array}{c}\text { The same as Inthavong et } \\
\text { al., } 2006 .\end{array}$ & $\begin{array}{l}\text { FLUENT6.1.2; Laminar flow; } \\
\text { Lagrangian particle tracking method }\end{array}$ & $\begin{array}{c}10 \mathrm{~L} / \mathrm{min} ; 3,6.2,9.1 \\
11.9,16,20,25,30 \\
39.2,48.3, \text { and } \\
55 \mu \mathrm{m}, 560,590 \\
930 \mathrm{Kg} / \mathrm{m}^{3}\end{array}$ & Smooth wall \\
\hline $\begin{array}{l}\text { Shi et al., } \\
2007\end{array}$ & $\begin{array}{l}\text { MRI scans of a 53-year- } \\
\text { old non-smoking } \\
\text { Caucasian male }\end{array}$ & $\begin{array}{l}\text { CFX 10; Laminar flow; Lagrangian } \\
\text { particle tracking algorithm }\end{array}$ & $\begin{array}{c}7.5 \text { and } 20 \mathrm{~L} / \mathrm{min} ; \\
1 \sim 50 \mu \mathrm{m} ; \\
1000 \mathrm{Kg} / \mathrm{m}^{3}\end{array}$ & $\begin{array}{l}\text { Smooth wall } \\
\text { and wall with a } \\
0.2 \mathrm{~mm} \text { wall } \\
\text { roughness layer }\end{array}$ \\
\hline $\begin{array}{l}\text { Shanley et } \\
\text { al., } 2008\end{array}$ & $\begin{array}{c}\text { MRI scans of an } \\
\text { anonymous, healthy, adult } \\
\text { male human subject }\end{array}$ & $\begin{array}{l}\text { FLUENT; Laminar flow; Lagrangian } \\
\text { particle tracking algorithm:discrete- } \\
\text { phase model (DPM) within FLUENT }\end{array}$ & $\begin{array}{c}2,3,4,5,6 \text {, and } \\
7 \mathrm{~L} / \mathrm{min} ; 1-10 \mu \mathrm{m} ; \\
1000,2000, \text { and } \\
3000 \mathrm{Kg} / \mathrm{m}^{3}\end{array}$ & $\begin{array}{l}\text { Smooth wall; } \\
\text { particles } \\
\text { deposit when } \\
\text { touch the wall }\end{array}$ \\
\hline
\end{tabular}

Figure 2.8 shows results of previous deposition simulations of several other researchers (Inthavong et al., 2006; Tian et al., 2007; Shi et al., 2007; Shanley et al., 2008). Although the nasal cavity models of cited works were generated from different subjects (Inthavong et al., 2006 and Tian et al., 2007: CT scans of a 25-year-old healthy, non-smoking Asian male; Shi et al., 2007: MRI scans of a 53-year-old non-smoking Caucasian male originating from Swift (1991); Shanley et al., 2008: MRI scans of an 
anonymous, healthy, adult male human subject), these results show similar trends. While for larger impaction parameters $\left(\mathrm{d}_{\mathrm{a}}^{2} \mathrm{Q}>1000\right)$, differences in the values of deposition fraction at fixed values of the impaction parameter are not insignificant (especially or the simulations of Shanley et al., 2008 and Tian et al., 2007), indicating the importance of inter-subject variability.

For larger impaction parameters $\left(\mathrm{d}_{\mathrm{a}}{ }^{2} \mathrm{Q}>1000\right)$, the numerical simulations results show under-prediction to some extent when compared with previous in vitro deposition data (see Figure 2.8). This difference is likely attributable to an effect of wall roughness. In the numerical simulation, the boundary condition for the wall of nasal cavity was specified as smooth, whereas there is a small but unavoidable wall roughness along the inner wall of the in vitro model due to manufacturing (see Kelly et al., 2004). Shi et al. (2007) also took the wall roughness into account using a $0.2 \mathrm{~mm}$ wall roughness layer, and the simulated results showed that this wall roughness height could enhance the particle deposition. 


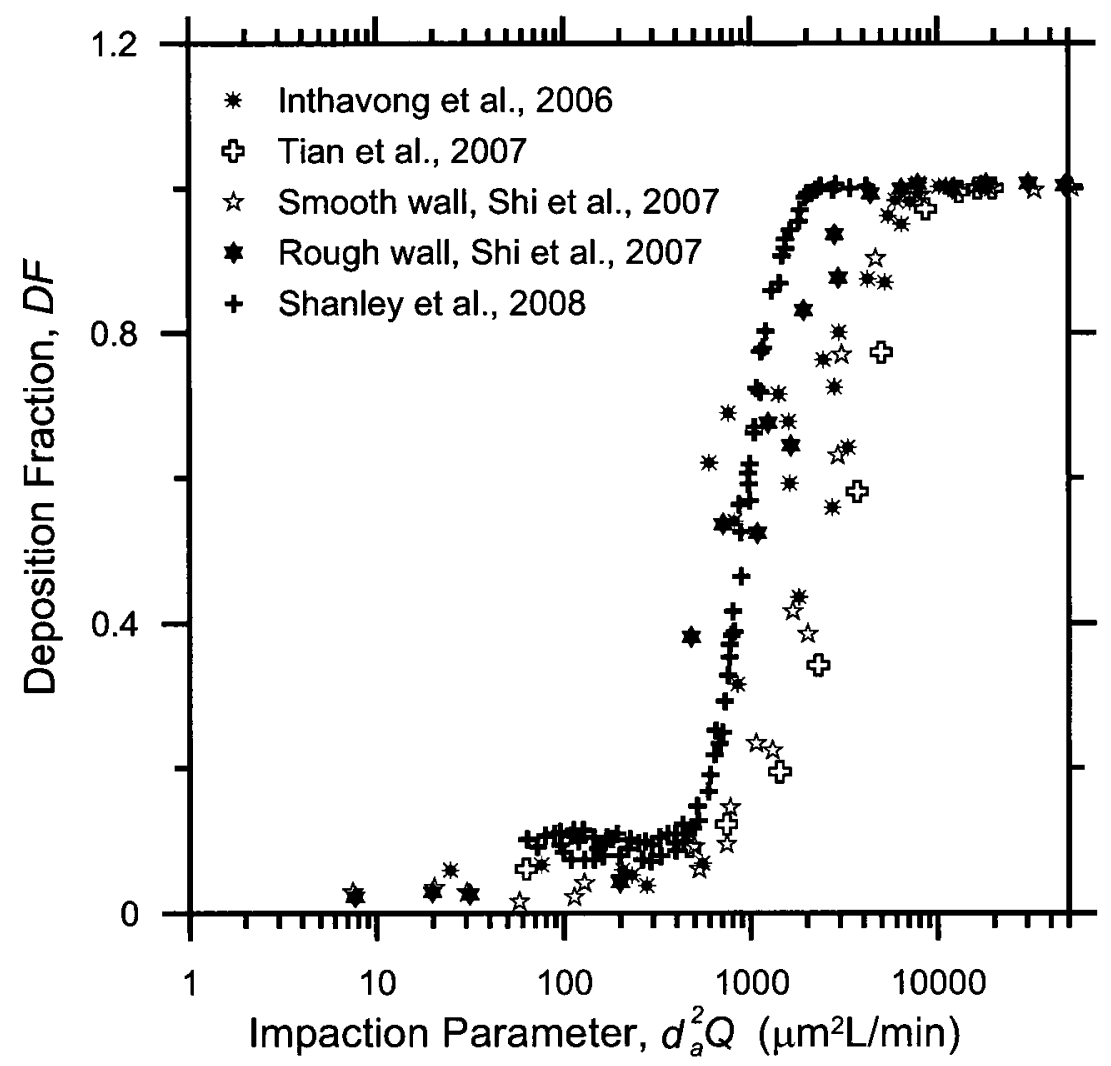

Figure 2.8 Previous simulation results of particle deposition fraction

\subsection{Geometry Related Limitations of Previous Work}

As discussed above, many efforts have been made to measure characteristics dimensions of human nasal cavity and to create 3D human nasal cavity models, and many experimental (in vivo and in vitro) and numerical studies have been conducted on particle deposition in the human nasal passages. Although these studies have shown that deposition fraction is mainly a function of particle size and density, airflow rate and nasal airway geometry, these studies are somewhat limited and difficult to compare since they were all completed using geometries of individual human subjects. To the author's knowledge, no previous investigations have been performed in a standardized human 
nasal geometry representing the general population in the context of aerosol medication deposition. Moreover, detailed numerical and experimental investigation of regional particle deposition, which is a significant issue in terms of improving nasal drug delivery techniques, has not been shown in previous studies. 


\section{Chapter 3: Construction of realistic models of the human nasal cavity}

The material presented in this chapter has been published in a refereed journal article co-authored by the author of this thesis, her supervisors, and two collaborators at the Ottawa Civic Hospital as: Y. Liu, M.R. Johnson, E.A. Matida, S. Kherani, J. Marsan (2009) Creation of a Standardized Geometry of the Human Nasal Cavity, Journal of Applied Physiology, 106: 784-795, 2009. While the text was written jointly, the CT scan measurements and image processing of individual scans and models were performed by the author.

A model that realistically represents the human nasal cavity is indispensable for studying, both numerically and experimentally, the airflow and particle deposition patterns in the human nasal cavity. Although several investigations reviewed in Chapter 2 have been performed to measure basic characteristic dimensions of the human nasal cavity using acoustic rhinometry (AR) (Hilberg et al., 1989; Min and Jang., 1995; Cheng, K. H. et al., 1996; Corey et al., 1997; Kesavanathan and Swift, 1998; Terheyden et al. 2000), magnetic resonance imaging (MRI) (Cheng, Y. S. et al., 1996; Cheng, K. H. et al., 1996; Corey et al., 1997; Guilmette et al., 1997), computed tomography (CT) (Min and Jang, 1995; Terheydenn et al. 2000), and using an in vivo cast (Swift and Kesavanatha.,1996), no previous researchers have published a median human nasal geometry that can be used to study airflow patterns and aerosol deposition patterns. Thus, the creation of a standardized three-dimensional human nasal cavity geometry which can represent the general population, as well as the establishment of a procedure for developing geometries of specific population subsets, would be an important and significant development that could benefit the research of nasal drug delivery as well as many other fields, such as nasal surgery, nasal toxicology, risk assessment, and disease diagnosis. 


\subsection{Baseline Data Used to Create a Standardized Human Nasal Cavity $\underline{\text { Model }}$}

Research to develop a standardized human nasal cavity model was completed in cooperation with the department of Otolaryngology at the Ottawa Hospital, Civic campus, which provided coronal CT scans for thirty healthy adult subjects whose nasal cavities were considered as normal and without any anatomic abnormalities. In keeping with the hospital's code of ethics all subjects, from whom the CT scans were obtained, remained anonymous throughout the entire study.

All scans were reviewed carefully and confirmed by a doctor of the department of Otolaryngology at the Ottawa Civic Hospital to have nasal anatomy considered to be within normal limits. Specifically, there were no obstructing lesions and the septum was not deviated. When the CT scans were taken, all subjects were in normal condition and decongested.

The age and gender of each subject were recorded as well as the characteristics of CT scans; this information is summarized in Table 3.1. The age of subjects ranged from 17 to 78 years (median of 45 ); there were 13 males and 17 females. Ethnicity was not specifically tracked as part of the original examinations and thus these data were not available. However, the included scans were chosen randomly and indiscriminately to include all available normal scans of patients presenting at the department of Otolaryngology. Thus, even though there was no explicit information regarding the ethnicity of the subjects, the ethnic backgrounds could be expected to comprise an 
unbiased sample of the Ottawa, Ontario, Canada population (predominantly Caucasian with $16 \%$ visible minorities including $4.0 \%$ Black, $2.9 \%$ Chinese, and $2.5 \%$ Arab as reported by Statistics Canada, 2008). The distance between consecutive slices was 2.5 $\mathrm{mm}$, except in 3 subjects for which cuts were 1 or $2 \mathrm{~mm}$ apart. The resolution of CT scans varied from 0.24 to 0.348 in $\mathrm{mm} / \mathrm{pixel}$.

Table 3.1 Information about the subjects and CT scan of 30 individuals in current study

\begin{tabular}{|c|c|c|c|c|c|}
\hline Subject No. & Gender & Age & $\begin{array}{l}\text { Number of } \\
\text { scan } \\
\text { planes }\end{array}$ & \begin{tabular}{|l|}
$\begin{array}{l}\text { Distance } \\
\text { between slices } \\
\text { (mm) }\end{array}$ \\
\end{tabular} & \begin{tabular}{|l|} 
Resolution \\
in x-y plane \\
(mm/pixel)
\end{tabular} \\
\hline 1 & $F$ & 45 & 38 & 2.5 & 0.325 \\
\hline 2 & $\mathrm{M}$ & 59 & 40 & 2.5 & 0.325 \\
\hline 3 & $F$ & 63 & 39 & 2.0 & 0.24 \\
\hline 4 & $F$ & 36 & 38 & 2.5 & 0.325 \\
\hline 5 & $F$ & 20 & 40 & 2.5 & 0.325 \\
\hline 6 & $\mathrm{~F}$ & 49 & 36 & 2.0 & 0.325 \\
\hline 7 & $\mathrm{~F}$ & 30 & 32 & 2.5 & 0.325 \\
\hline 8 & $M$ & 74 & 43 & 2.5 & 0.325 \\
\hline 9 & $M$ & 60 & 42 & 2.5 & 0.325 \\
\hline 10 & $F$ & 17 & 39 & 2.5 & 0.325 \\
\hline 11 & $M$ & 56 & 40 & 2.5 & 0.325 \\
\hline 12 & $M$ & \begin{tabular}{|l|}
52 \\
\end{tabular} & 44 & 2.5 & \begin{tabular}{|l|}
0.325 \\
\end{tabular} \\
\hline 13 & $M$ & 39 & 40 & 2.5 & 0.325 \\
\hline 14 & $\mathrm{~F}$ & 37 & 38 & 2.5 & 0.325 \\
\hline 15 & $M$ & 52 & 43 & 2.5 & 0.3 \\
\hline 16 & $M$ & 41 & 39 & 2.5 & 0.293 \\
\hline 17 & $\mathrm{~F}$ & 78 & 43 & 2.5 & 0.339 \\
\hline 18 & $F$ & 26 & 41 & 2.5 & 0.245 \\
\hline 19 & $M$ & 52 & 42 & 2.5 & 0.283 \\
\hline 20 & $\mathbf{F}$ & 23 & 44 & 2.5 & 0.313 \\
\hline 21 & $M$ & 52 & 39 & 2.5 & 0.325 \\
\hline 22 & $F$ & 45 & 34 & 2.5 & 0.325 \\
\hline 23 & $F$ & 52 & 38 & 2.5 & 0.325 \\
\hline 24 & $F$ & 30 & 45 & 2.5 & 0.325 \\
\hline 25 & $F$ & 34 & 41 & 2.5 & 0.325 \\
\hline 26 & $F$ & 66 & 80 & 1.0 & 0.348 \\
\hline 27 & $F$ & 49 & 42 & 2.5 & 0.325 \\
\hline 28 & $M$ & \begin{tabular}{|l}
36 \\
\end{tabular} & 40 & 2.5 & \begin{tabular}{|l|l}
0.325 \\
\end{tabular} \\
\hline 29 & $M$ & 42 & 47 & 2.5 & 0.293 \\
\hline 30 & $M$ & 45 & 40 & 2.5 & 0.293 \\
\hline \multicolumn{2}{|l|}{ AVERAGE } & 45.3 & 41.57 & 2.417 & 0.315 \\
\hline \multicolumn{2}{|l|}{ MEDIAN } & 45 & 40 & 2.5 & 0.325 \\
\hline \multicolumn{2}{|l|}{ MAX } & 78 & 80 & 2.5 & 0.348 \\
\hline \multicolumn{2}{|l|}{$\overline{M I N}$} & 17 & 32 & 1 & 0.24 \\
\hline
\end{tabular}




\subsection{Generation and alignment of individual 3D nasal cavity models}

\subsubsection{Construction of coarse 3D models from CT scans}

To construct models from CT scans, first, all sets of raw 2D CT images were processed using 3D-DOCTOR (Able Software Corp., Lexington, MA, USA) to produce coarse, 3D nasal cavity geometries (including both left and right nasal airways) for each of the subjects. The procedure is illustrated in Figure 3.1 and is described below:

(a) Images were imported and calibration parameters were set (i.e. image resolution and distance in between scans), as shown in Figure 3.1a. Three spatial coordinates, $x, y$, $z$, were used in the 3D-DOCTOR software. The correct setting of image resolution ensured the real size of each CT scan, and the distance in between scans was used to get the correct length along $\mathrm{z}$ direction.

(b) Images were segmented (using the texture-based auto-segmentation function within 3D-DOCTOR software) and boundaries were processed, as shown in Figure 3.1.b. In present work, the paranasal sinuses (the air-filled pockets located within the bones of the skull surrounding the nasal cavity) were excluded from the nasal model. The segmenting parameter of 3D-DOCTOR software was adjusted to make the boundaries of the paranasal sinuses distinct from those of the main nasal passageway.

(c) Surfaces were rendered and coarse 3D models were exported (as shown in Figure 3.1.c). 


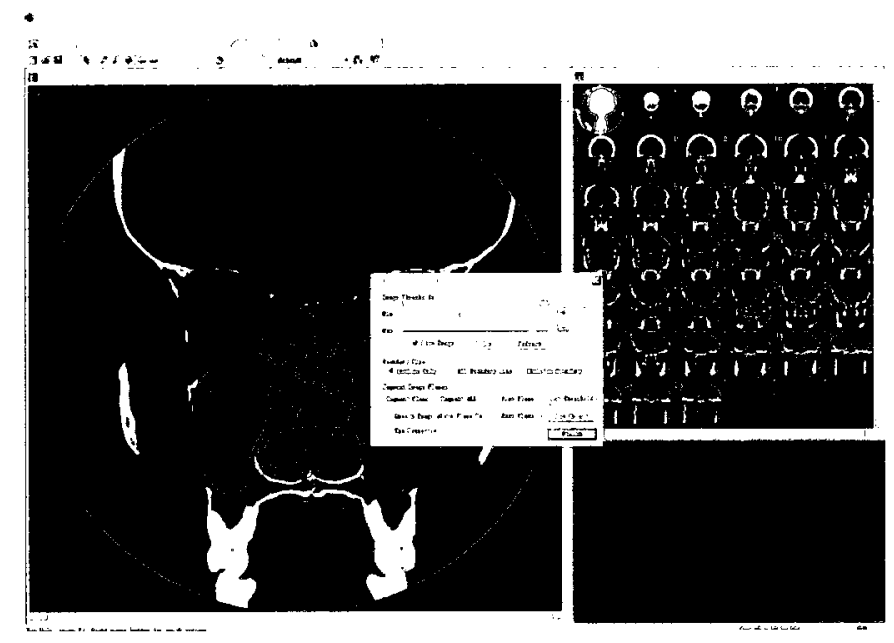

$\mathbf{a}$

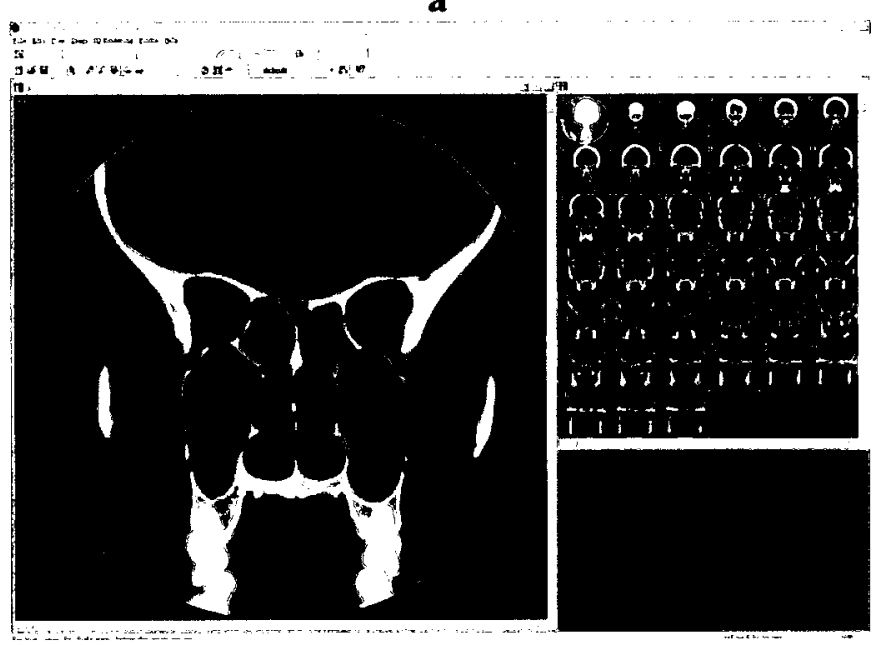

b

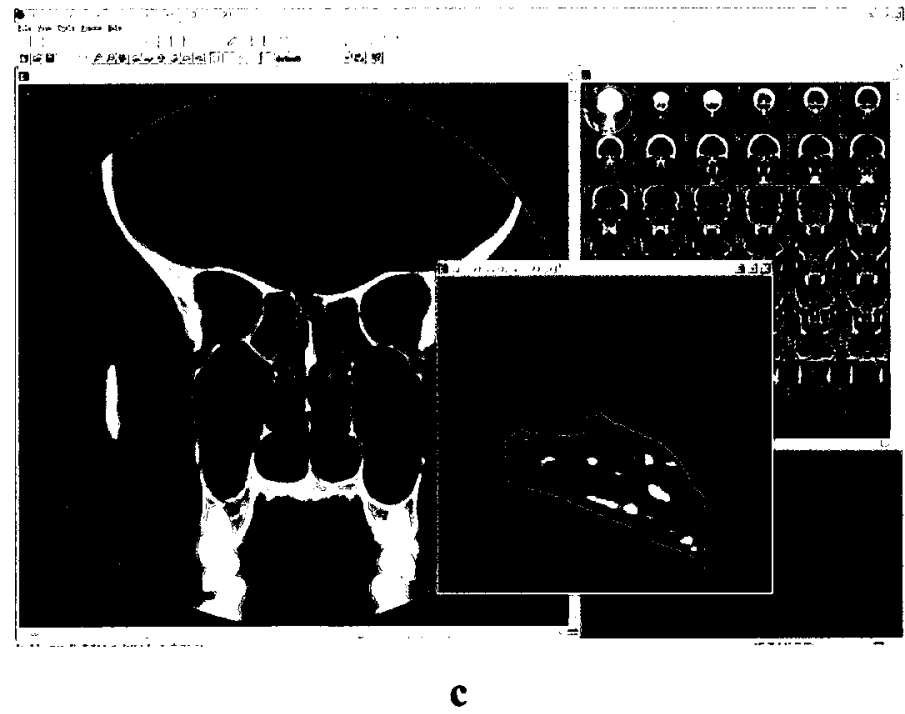

Figure 3.1 Procedure of generation of coarse nasal cavity model using 3D-Doctor software 
In present study, the main nasal passage is the only domain we are interested in. Thus, the paranasal sinuses are excluded. During processing of the raw CT scans, the paranasal sinuses were easily distinguished from the main nasal passageway and excluded by adjusting the segmenting parameter within the 3D-DOCTOR software.

\subsubsection{Rotational alignment of coarse $3 D$ models}

In general, CT scans are taken in three basic orientations: coronal, transverse (also called axial) or sagittal. In the present study, the CT scans offered by the hospital are all in the coronal plane. There is no precise method of positioning subjects or defining a measurement origin, thus the $3 \mathrm{D}$ orientation of each set of $\mathrm{CT}$ scans varies. It can be noticed from Figure 3.2 that there is an apparent rotational misalignment toward the clockwise direction of the sample raw $\mathrm{CT}$ images.

Before direct comparisons or physical measurements can be made, all of the generated coarse 3D models needed to be rotated and aligned to a commonly defined position. Sufficient geometric constraints were applied to satisfy six degrees of freedom (e.g., three spatial coordinates, $\mathrm{x}, \mathrm{y}, \mathrm{z}$ of a common origin and three independent rotation angles about this origin). A multistep procedure was developed for providing the necessary geometric constraints and will be explained in detail in the following sections. 


\subsubsection{Specifying the landmark and choana from the raw CT scans}

First, a common "landmark" on each set of CT scans was determined. The tip of the anterior maxillary spine, or the point where the nasal septum intersects the maxilla bone, was chosen as the first landmark (Figure 3.2.a). This location was identifiable on each set of coronal plane CT scans and was representative of the anterior region of the nose. The mean length of the anterior maxillary spine of all 30 subjects was determined to be $6.5 \pm 1.9 \mathrm{~mm}$. Thus, the uncertainty in defining this location was smaller than the spacing between CT scans. The landmark was identified on each model and assigned an arbitrary $x-y-z$ coordinate location of $(0 \mathrm{~mm}, 0 \mathrm{~mm}, 0 \mathrm{~mm})$, thus removing three degrees of freedom. A second constraint was developed by identifying the point on a posterior coronal plane where the two nasal airways merge, known as the choana or the opening into the nasopharynx (Figure 3.2.b). The imaginary line joining the anterior maxillary spine and the choana was then used to define the nominal plane of symmetry.

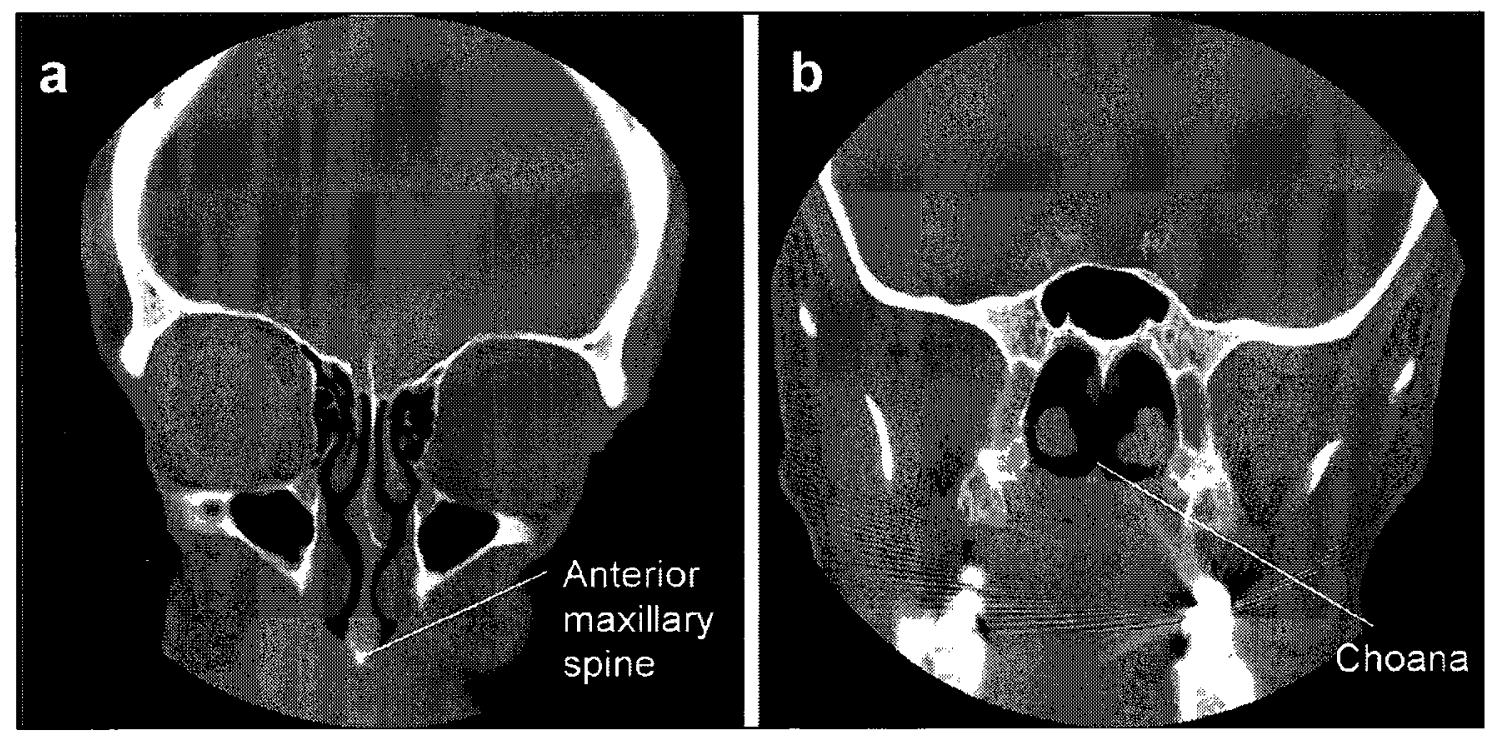

Figure 3.2 Identification of the (a) maxillary spine landmark and (b) choana in the raw, coronal plane $\mathrm{CT}$ scans 


\subsubsection{Rotating coarse nasal models}

The procedure for rotational alignment is illustrated schematically using model No.8 as example in Figure 3.3, which shows the initial (x-y-z) and final, aligned (X-Y-Z) coordinate axes of the coarse nasal model.

The first step of rotational alignment was to rotate the $3 \mathrm{D}$ model about the line joining the anterior maxillary spine and the choana until the projected area viewed from the top of the model was minimized, and the $x-y$ plane of symmetry could be fully specified for each model, thus removing two further degrees of freedom. This initial rotation about the imaginary reference line linking the maxillary spine landmark and the choana is indicated by the angle, $\alpha$, as shown in Figure 3.3. The secondary rotation about the $\mathrm{y}$-axis is given by angle, $\beta$. After the first two rotations, the symmetry plane was aligned with the final X-Y coordinate plane.

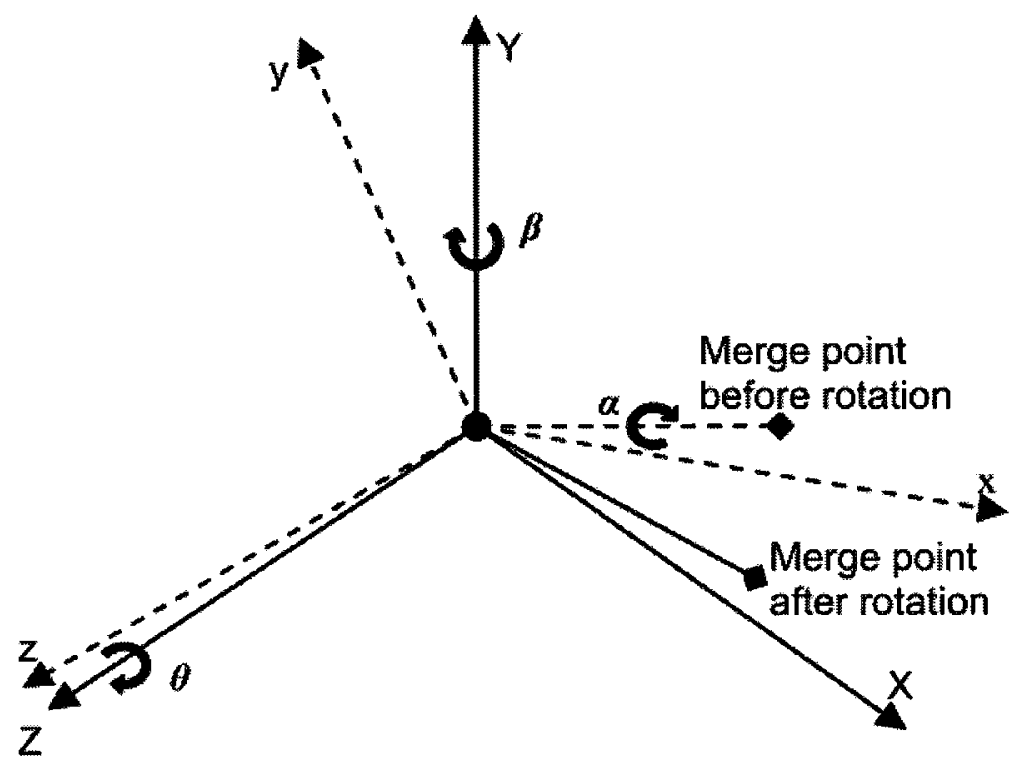

Figure 3.3 Coordinate system for rotating the coarse 3D nasal cavity model. Example images show coarse model 8 
The procedure for the first two rotations is shown in Figure 3.4.a, b, and c, which shows rendered images of nasal model No. 8 before and after the first two rotations. As previously mentioned, all subjects in the current study were aligned approximately to obtain CT scans in the coronal plane. Thus, the magnitudes of the rotational transformations $\alpha$ and $\beta$ were small. In the current data, the maximum rotational corrections, $\alpha$ in the coronal $(\mathrm{y}-\mathrm{z})$ plane, and $\beta$ in the transverse $(\mathrm{x}-\mathrm{z})$ plane, relative to the orientation of the raw CT scans were all between $-5.0^{\circ}$ and $4.0^{\circ}$ and $-2.0^{\circ}$ and $5.0^{\circ}$ respectively. Even though the magnitude of $\alpha$ and $\beta$ were small, the first two steps of the rotation were indispensable due to the small size and complexity of the human nasal cavity.
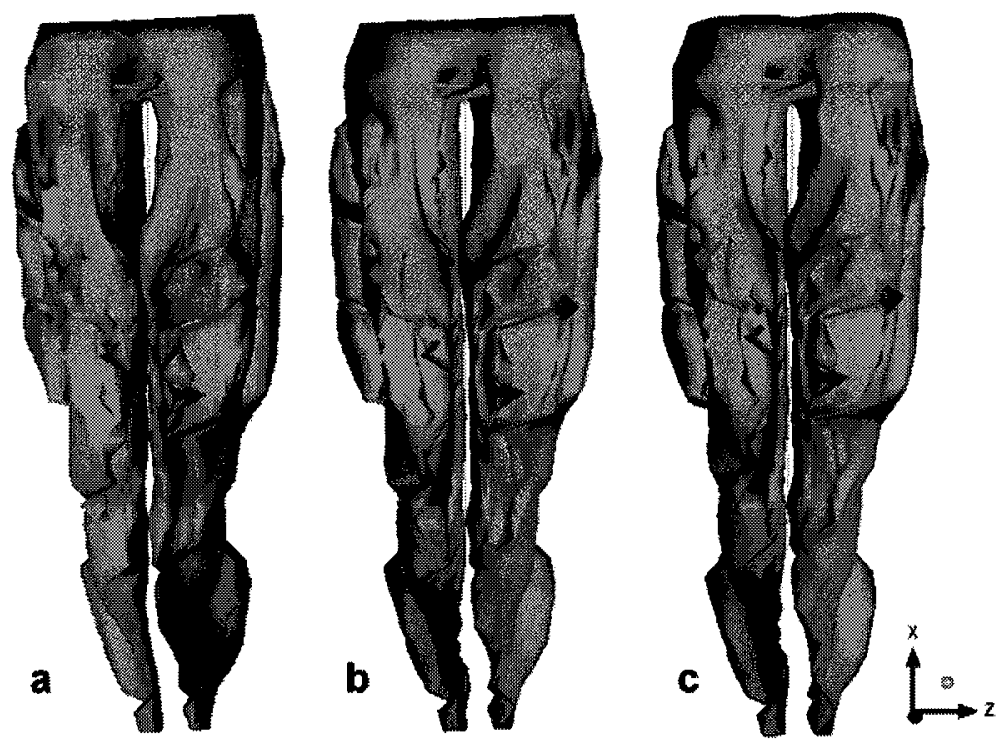

Figure 3.4 Illustration of the first two steps of rotational alignment of a coarse 3D nasal cavity model. (Example images show coarse model 8.) (a) Top view of model before rotation; (b)Top view of model after rotation around imaginary reference line linking the maxillary spine landmark and the posterior merge point; (c)Top view of model after rotation around $\mathrm{Y}$-axis to align symmetry plane with $\mathrm{X}$-axis 
The final step of rotational alignment was rotating the model about an axis perpendicular to the plane of symmetry (Z-axis) until the projected area on $y-z$ plane as viewed from the front of the model was minimized. This rotation is indicated by the angle, $\theta$, in Figure 3.4, and an example is shown in Figure 3.5 for model No. 8. $\theta$ depends on the rotational pitch of each subject's head during the CT scans, and varied more significantly than $\alpha$ and $\beta$ among subjects. For the current data, the maximum value of $\theta$ was 35.5 degrees.

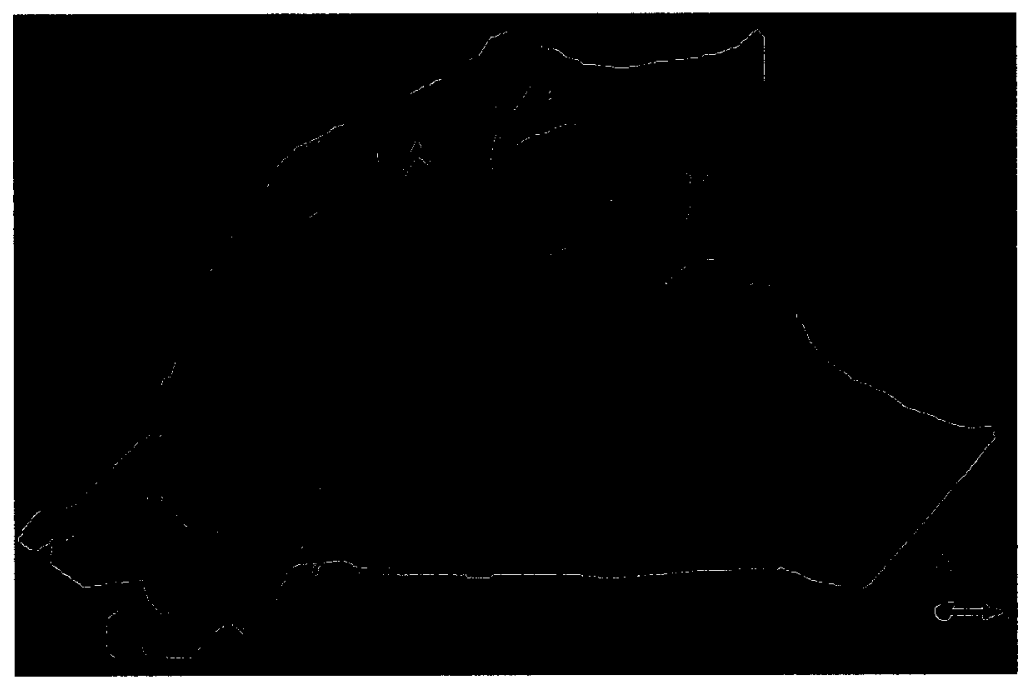

Figure 3.5 Example images of model number 8 showing rotational alignment of a coarse 3D nasal cavity model. Original orientation is shown in green and final, aligned model is shown in red. The positions of the imaginary reference lines before and after alignment are indicated

All 3D coarse nasal models were aligned using this procedure. With the six degrees of freedom appropriately constrained, the location of the common maxillary spine landmark of each subject was specified, and the direction of the $\mathrm{X}-\mathrm{Y}-\mathrm{Z}$ axes was fixed relative to the nominal plane of symmetry. 


\subsection{Creation of the standardized human nasal cavity model}

\subsubsection{Measuring characteristic length and scaling individual coarse models}

In order to determine a median shape of a standardized 3D nasal cavity, it was necessary to compensate for size variations among subjects, which is difficult because of the high complexity and diversity of the nasal cavity. Before attempting to determine the median geometry of the standardized human nasal cavity model, all coarse aligned nasal cavity models were divided into three sub-regions: the anterior/nostril region (including the part from anterior tip of the nostril to the anterior maxillary spine), the middle/nasal airway region (from the maxillary spine to the choana), and the posterior region (corresponding to the region posterior to the choana known as the nasopharynx), as indicated in Figure 3.6 .

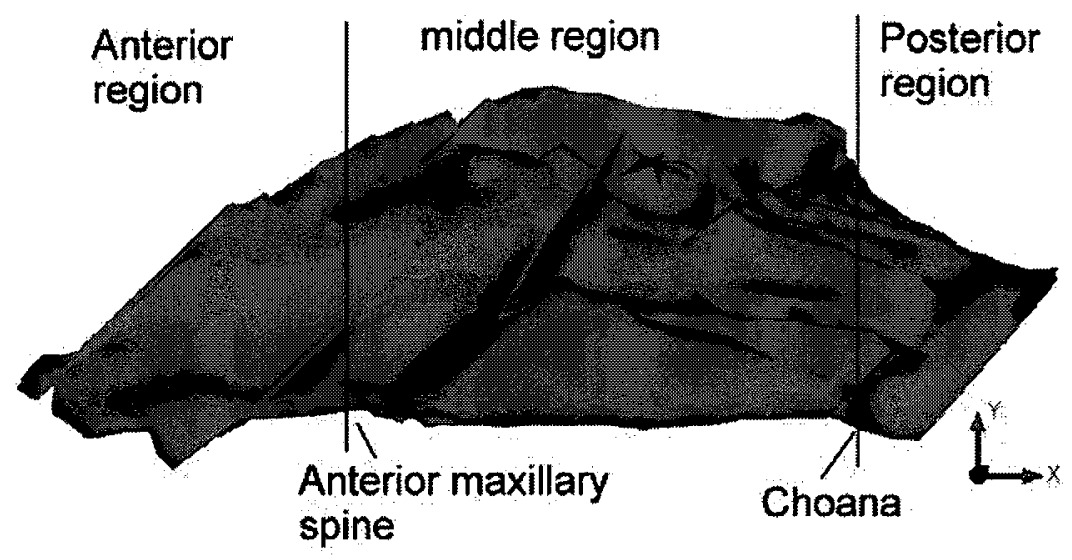

Figure 3.6 Three sub-regions of a 3D nasal cavity

For each subject, a characteristic length of the middle/airway region was determined. The characteristic length was defined as the dimension in the X-direction between the anterior maxillary spine landmark to the choana or posterior merge point of the nasal 
airways. As listed in Table 3.2, the measured characteristic lengths ranged from $50 \mathrm{~mm}$ to $80.5 \mathrm{~mm}$, which reflects gender differences as well as normal variability among subjects. Relative to the average length of $65.1 \mathrm{~mm}$, the standard deviation was $7.2 \mathrm{~mm}$ or $11 \%$.

Table 3.2 Measurements of geometric parameters of the middle region of aligned scaled coarse nasal models

\begin{tabular}{|c|c|c|c|c|c|c|}
\hline \multirow[t]{2}{*}{$\begin{array}{l}\text { Subject } \\
\text { No. }\end{array}$} & \multirow[t]{2}{*}{ Gender } & \multirow{2}{*}{\begin{tabular}{|l|} 
Characteristic \\
length of middle \\
region in X- \\
direction $(\mathrm{mm})$
\end{tabular}} & \multicolumn{2}{|c|}{$\begin{array}{l}\text { Internal volume of } \\
\text { middle region of single } \\
\text { nasal passage, }\left(\mathrm{mm}^{3}\right)\end{array}$} & \multicolumn{2}{|c|}{$\begin{array}{l}\text { Cross-sectional area in } \\
\text { middle region of a single } \\
\text { passage, }\left(\mathrm{mm}^{2}\right)\end{array}$} \\
\hline & & & Left & Right & Max. & Min. \\
\hline 1 & $F$ & 60.1 & 10418 & 10509 & 240 & 111 \\
\hline 2 & $M$ & 67.6 & 10212 & 11245 & 209 & 68 \\
\hline 3 & $F$ & 50 & 8745 & 7523 & 215 & 118 \\
\hline 4 & $F$ & 56.7 & 8934 & 10000 & 307 & 69 \\
\hline 5 & $F$ & 69.3 & 6468.2 & 8075 & 179 & 52 \\
\hline 6 & $F$ & 60.8 & 10462 & 6782 & 221 & 52 \\
\hline 7 & $\mathrm{~F}$ & 62.9 & 6846 & 7660 & 157 & 67 \\
\hline 8 & $M$ & 66.3 & 10862 & 9756 & 275 & 68 \\
\hline 9 & $M$ & 65.1 & 10481 & 11880 & 244 & 67 \\
\hline 10 & $\mathrm{~F}$ & 67.9 & 9315 & 8988 & 224 & 71 \\
\hline 11 & $M$ & 64.6 & 10980 & 12561 & 238 & 103 \\
\hline 12 & $M$ & 73.4 & 11227 & 11827 & 242 & 85 \\
\hline 13 & $M$ & 60.1 & 16145 & 13819 & 354 & 82 \\
\hline 14 & $F$ & 60.8 & 9971 & 9486 & 230 & 82 \\
\hline 15 & $M$ & 57.7 & 8655 & 13601 & 274 & 82 \\
\hline 16 & $M$ & 61 & 7066 & 8111 & 202 & 60 \\
\hline 17 & $F$ & 65 & 10527 & 9331 & 213 & 91 \\
\hline 18 & $\mathrm{~F}$ & 60 & 7567 & 9104 & 254 & 50 \\
\hline 19 & $M$ & 78.3 & 15549 & 14289 & 280 & 78 \\
\hline 20 & $\mathrm{~F}$ & 65.3 & 12099 & 14356 & 296 & 97 \\
\hline 21 & $M$ & 72.3 & 12807 & 16192 & 312 & 89 \\
\hline 22 & $\mathrm{~F}$ & 65.6 & 8093 & 7199 & 189 & 59 \\
\hline 23 & $F$ & 63.8 & 8346 & 9774 & 194 & 58 \\
\hline 24 & $F$ & 77.4 & 11293 & 9285 & 230 & 43 \\
\hline 25 & $F$ & 61.2 & 11843 & 12411 & 260 & 102 \\
\hline 26 & $F$ & 53 & 10747 & 10751 & 322 & 83 \\
\hline 27 & $F$ & 66.3 & 9127 & 10432 & 236 & 68 \\
\hline 28 & $M$ & 63.6 & 9951 & 16435 & 306 & 89 \\
\hline 29 & $M$ & 80.5 & 15989 & 11660 & 261 & 66 \\
\hline \multirow[t]{6}{*}{30} & $M$ & 76.6 & 12283 & 11002 & 223 & 66 \\
\hline & AVERAGE & 65.1 & 10434 & 10801 & 246 & 76 \\
\hline & MEDIAN & 64.8 & 10440 & 10471 & 239 & 70 \\
\hline & STD. DEV. & 7.2 & 2458 & 2568 & 46 & 19 \\
\hline & MAX & 80.5 & 16145 & 16435 & 354 & 118 \\
\hline & MIN & 50 & 6468 & 6782 & 157 & 43 \\
\hline
\end{tabular}


To compensate for size variations and to facilitate computation of a median 3D shape, each of the nasal cavity models was geometrically scaled using the ratio of the averaged characteristic length of all nasal geometries to the characteristic length of that specific geometry:

$$
S_{i}=\bar{L}_{M} / L_{M, i}
$$

where $S_{i}$ is the geometric scale factor for the ith model, $\bar{L}_{M}=65.1 \mathrm{~mm}$ and is the averaged characteristic length, and $L_{M, i}$ is the characteristic length of the ith model. Table 3.2 lists geometric parameters of the middle region of aligned scaled coarse nasal models. Most of the scale factors were within $11 \%$ and all were within $24 \%$ of unity, which clearly shows that the scaling process was generally a small adjustment.

\subsubsection{Slicing and smoothing individual coarse models}

In addition to the process of aligning and scaling the models, each of the aligned and scaled coarse 3D models was sliced by $\mathrm{Y}-\mathrm{Z}$ planes along the $\mathrm{X}$-direction using ICEM software (Ansys Inc., Canonsburg, PA, USA) to generate up to 56 coronal plane images spaced $2 \mathrm{~mm}$ apart for each of the 30 full nasal models.

As noted previously, the right and left nasal passages of each of the 30 full nasal models in the present study were considered as distinct geometries, thereby increasing the number of subjects. Thus, in the frontal and middle regions of the model (anterior to the choana), each of the coronal plane images generated from the aligned and scaled models was separated into two distinct images containing the boundaries of either the left or right 
nasal passages. The images of the left nasal passages were mirrored about the plane of symmetry (determined using the procedure described above), to produce thirty additional sets of images of right nasal airways. This procedure effectively doubled the available sample size to produce a total sample of 60 sets of right nasal airway coronal plane images.

Figure 3.7 shows a plot of the number of coronal plane images available at each $\mathrm{X}$ location along the length of the nasal cavity. The vertical lines on the graph indicate the relative X-locations of the entry into the final nasal model (explained below), the anterior maxillary spine, the choana, and the posterior termination of the final model (within the nasopharynx near the pharyngeal wall). Throughout most of the length of the nasal cavity, 60 cross-sectional (coronal) images were available, which included the region of highest geometric variability.

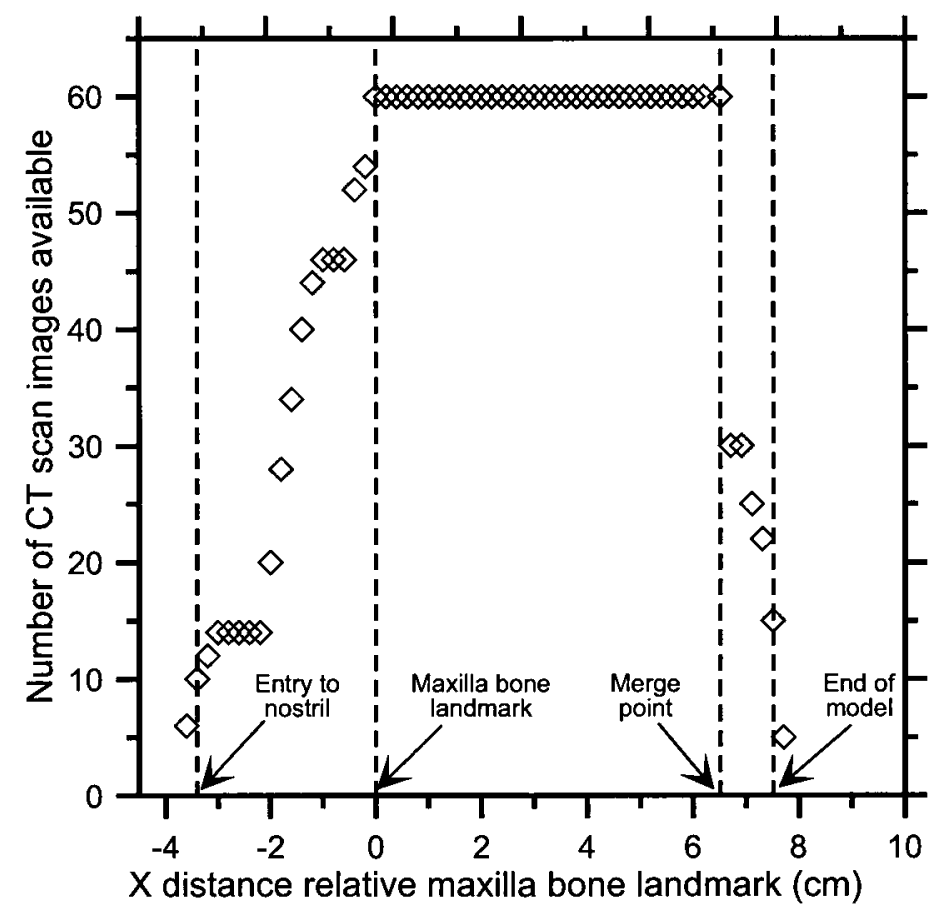

Figure 3.7 Number of available coronal-plane images at each X-location along nasal cavity 
Figure 3.7 illustrates that not all sets of available CT scans extended fully to the tip of the nose and in general they terminated at different locations posterior to the choana. Thus fewer than 60 coronal plane images were available toward the anterior and posterior ends of the model, which means information about the nostril of several nasal cavity models is missing. The solution to this problem will be discussed in following section.

Following the slicing procedure, the boundaries obtained from the 56 cross-sectional images of each coarse model were checked and smoothed using the ICEM software to eliminate any obvious errors generated from 3D-DOCTOR, such as discontinuous curves and/or abnormally sharp or angled edges. The smoothing procedure consisted of four steps as illustrated in Figure 3.8.

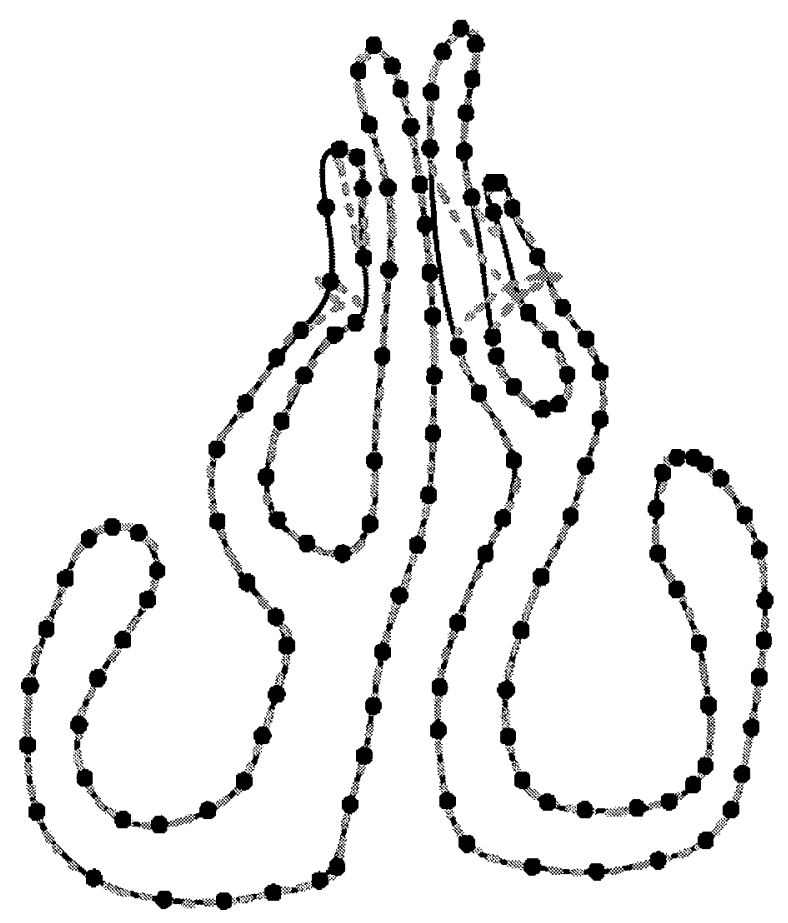

Figure 3.8 Example of cross-sectional boundary smoothing. The dashed grey curve is the original boundary, points are manually selected on this boundary, and the black continuous curve is the corrected and smoothed boundary 
(ii) 60 100 points along each boundary curve were selected. (The number of points varied for different lengths of boundary curves);

(iii) Smoothed boundary curves were created (see Figure 3.8) from these points using the automatic curve-generating function within ICEM, which uses the standard B-Spline algorithm (De Boor, 1978);

(iv) A set of 56 new, smoothed cross-sectional (coronal) images for each coarse model were exported;

(v) The smoothed 3D surfaces from the adjusted coronal images of each single passage were rendered using the surface-generating function within ICEM.

Figure 3.9 shows 32 samples of the 60 final coronal plane images at the location $30 \mathrm{~mm}$ posterior to the maxillary spine landmark in the nasal cavity. In these images, the geometry of the maxillary spine landmark region is the most dramatic of any location along the nasal cavity. Even though all the coronal images have many common characteristics, turbinates for example, their exact shape varies dramatically, again indicating the diversity of these subjects. 


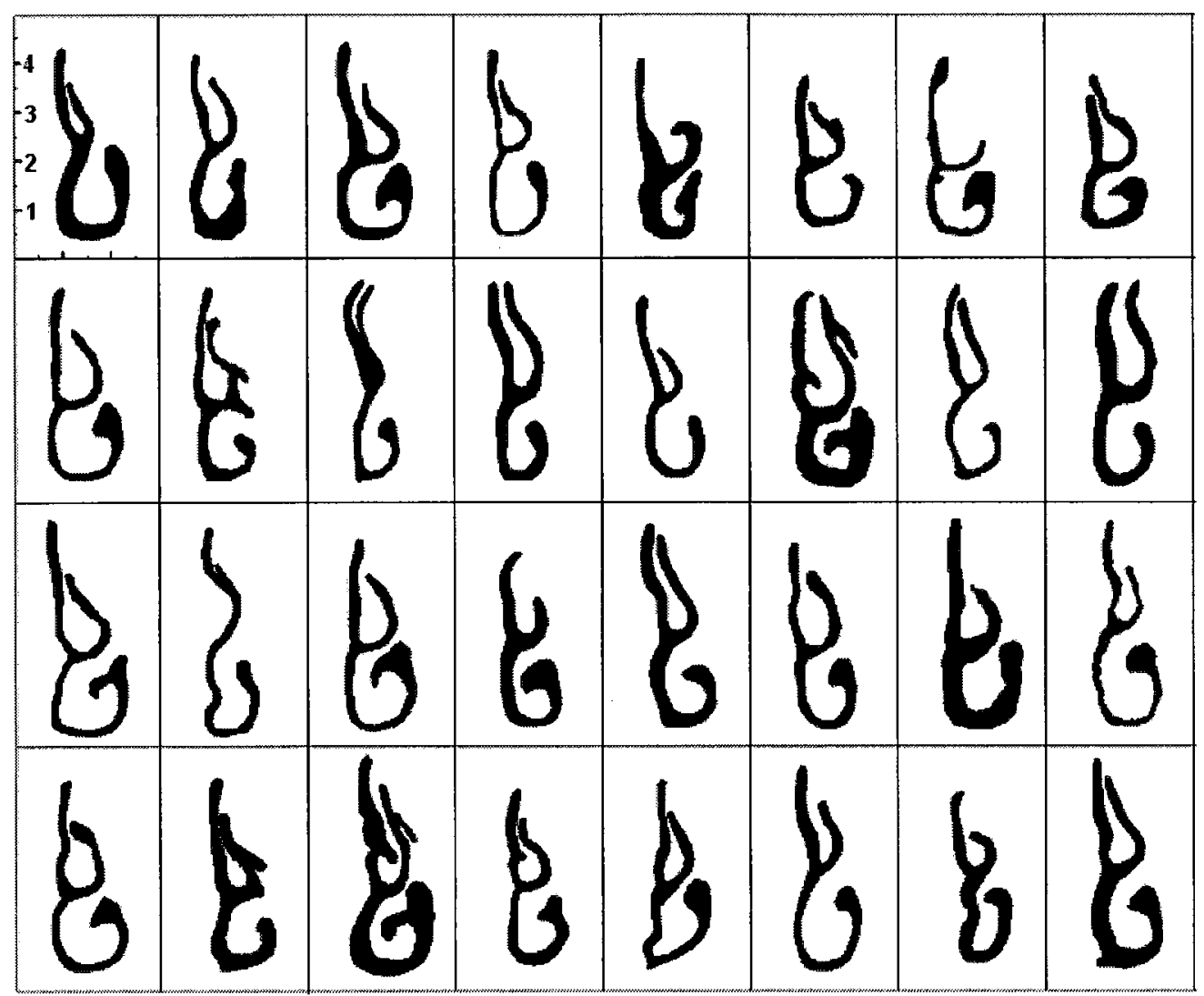

Figure 3.932 out of 60 nasal cavity cross-sectional shapes located $30 \mathrm{~mm}$ from the maxillary spine landmark

\subsubsection{Measurement of characteristic dimensions of individual coarse $3 D$ nasal cavity} models

As shown in Table 3.2, apart from characteristic length, several measurements (e.g. the internal volume and flow cross-sectional area of single nasal passage) were taken for each aligned scaled smoothed coarse model. The internal volume of the middle section of each single nasal airway was obtained by summing the products of the cross-sectional areas of the coronal planes (that fell in the middle region of the model) and the distance between each of the planes $(2 \mathrm{~mm})$. The internal volumes of each single nasal airway 
(i.e. left or right airway in the middle region) and the minimum and maximum flow cross-sectional areas in each airway showed significant variability among subjects. The maximum and minimum values of cross-sectional areas for extreme subjects were as much as $55 \%$ larger or $43 \%$ smaller than average values, which demonstrated the high diversity of the nasal model again.

Table 3.3 Measurements of frontal/nostril regions from 7 sets of CT scans

\begin{tabular}{|c|c|c|c|c|c|c|c|c|c|}
\hline \multirow[t]{2}{*}{$\begin{array}{c}\text { Subject } \\
\text { No. }\end{array}$} & \multirow[t]{2}{*}{ Gender } & \multirow{2}{*}{$\begin{array}{l}\text { Length of } \\
\text { frontal } \\
\text { region in } \\
x- \\
\text { direction } \\
\text { (cm) }\end{array}$} & \multirow{2}{*}{$\begin{array}{l}\text { Length } \\
\text { along major } \\
\text { axis of } \\
\text { nostril at } \\
\text { inlet plane } \\
\text { (cm) }\end{array}$} & \multirow{2}{*}{$\begin{array}{c}\text { Width } \\
\text { along } \\
\text { minor axis } \\
\text { of nostril } \\
\text { at inlet } \\
\text { plane }(\mathrm{cm})\end{array}$} & \multirow[t]{2}{*}{$\begin{array}{c}\text { Nostril } \\
\text { Angle } \\
\text { (degrees } \\
\text { ) }\end{array}$} & \multicolumn{2}{|c|}{$\begin{array}{c}\text { Internal volume of } \\
\text { single nasal } \\
\text { passage (nostril to } \\
\text { choana) }\left(\mathrm{mm}^{3}\right)\end{array}$} & \multicolumn{2}{|c|}{$\begin{array}{l}\text { Surface area of } \\
\text { single nasal } \\
\text { passage (nostril } \\
\text { to choana) }\left(\mathrm{cm}^{2}\right)\end{array}$} \\
\hline & & & & & & left & right & left & right \\
\hline 7 & $F$ & 3.56 & 2.35 & 0.92 & 65.22 & 10.1 & 10.8 & 85.1 & 85.0 \\
\hline 8 & M & 3.49 & 2.25 & 0.65 & 68.94 & 15.3 & 12.0 & 113.5 & 110.9 \\
\hline 9 & M & 3.24 & 2.64 & 0.74 & 70.08 & 13.6 & 15.3 & 107.9 & 101.1 \\
\hline 11 & $M$ & 3.42 & 2.10 & 0.87 & 69.76 & 14.8 & 16.3 & 102.2 & 104.2 \\
\hline 15 & M & 4.29 & 2.15 & 0.97 & 67.71 & 10.8 & 14.6 & 115.1 & 107.6 \\
\hline 23 & $F$ & 3.05 & 1.83 & 0.56 & 68.55 & 11.3 & 12.4 & 79.7 & 85.4 \\
\hline 27 & $F$ & 3.19 & 2.06 & 0.83 & 68.23 & 13.2 & 14.1 & 109.8 & 112.3 \\
\hline \multicolumn{2}{|c|}{ AVERAGE } & 3.46 & 2.2 & 0.79 & 68.36 & \multicolumn{2}{|l|}{13.18} & \multicolumn{2}{|l|}{101.4} \\
\hline \multicolumn{2}{|c|}{ STD.DEV. } & 0.41 & 0.25 & 0.15 & 1.61 & \multicolumn{2}{|l|}{1.97} & \multicolumn{2}{|l|}{12.28} \\
\hline \multicolumn{2}{|l|}{ MAX } & 4.29 & 2.64 & 0.97 & 70.08 & \multicolumn{2}{|l|}{16.25} & \multicolumn{2}{|l|}{79.74} \\
\hline \multicolumn{2}{|l|}{ MIN } & 3.05 & 1.83 & 0.56 & 65.22 & \multicolumn{2}{|l|}{10.13} & \multicolumn{2}{|l|}{115.05} \\
\hline
\end{tabular}

As stated previously, not all of the available sets of scans had information extending to the anterior tip of the nostrils. Table 3.3 summarizes measured dimensions for the anterior/nostril region of the nasal models using the available seven complete sets of scans which represent 14 single nasal passageways. The total volume and surface area of seven complete nasal cavities can be seen in Table 3.3. Similar to the internal volume measurement, the surface area of each nasal cavity was obtained by summing the product of the perimeter (i.e., the length of the outline of the image) and the inter-plane distance 
(i.e., $2 \mathrm{~mm}$ ) for each coronal plane. This was done for the entire length of the nasal cavity. As shown in Table 3.3, there is variability in the length and width of the nostrils as well as the angle between the major axes of the nostril in the entry plane, which is consistent with previous studies results by Kesavanathan and Swift (1998).

\subsubsection{Image processing methodology for generating median coronal plane images}

Because of the significant variability throughout the individual nasal cavities, a novel and sophisticated image processing protocol was created and adopted here to fulfill the goal of generating a meaningful standardized cavity model.

For each of the $56 \mathrm{X}$-locations, the coronal cross-sectional images were processed to produce a median cross-sectional shape at that location. Several in-house algorithms were developed to aid this process using image-processing tools from Labview (National Instruments Corporation, Austin, TX). Using physical shape and area as constraints, these algorithms allowed statistically based estimation of median cross-sectional geometries through a procedure of ensemble averaging, thresholding, and edge detection.

Figure 3.10 a-d shows various stages of the image processing using images for a coronal plane at $30 \mathrm{~mm}$ X-location relative to the maxillary spine landmark as example. This region of the nasal cavity exhibited the maximum geometric variability and presented the greatest challenge in determining a median shape. The full analysis procedure involved four steps as described below and shown in Figure 3.10. 

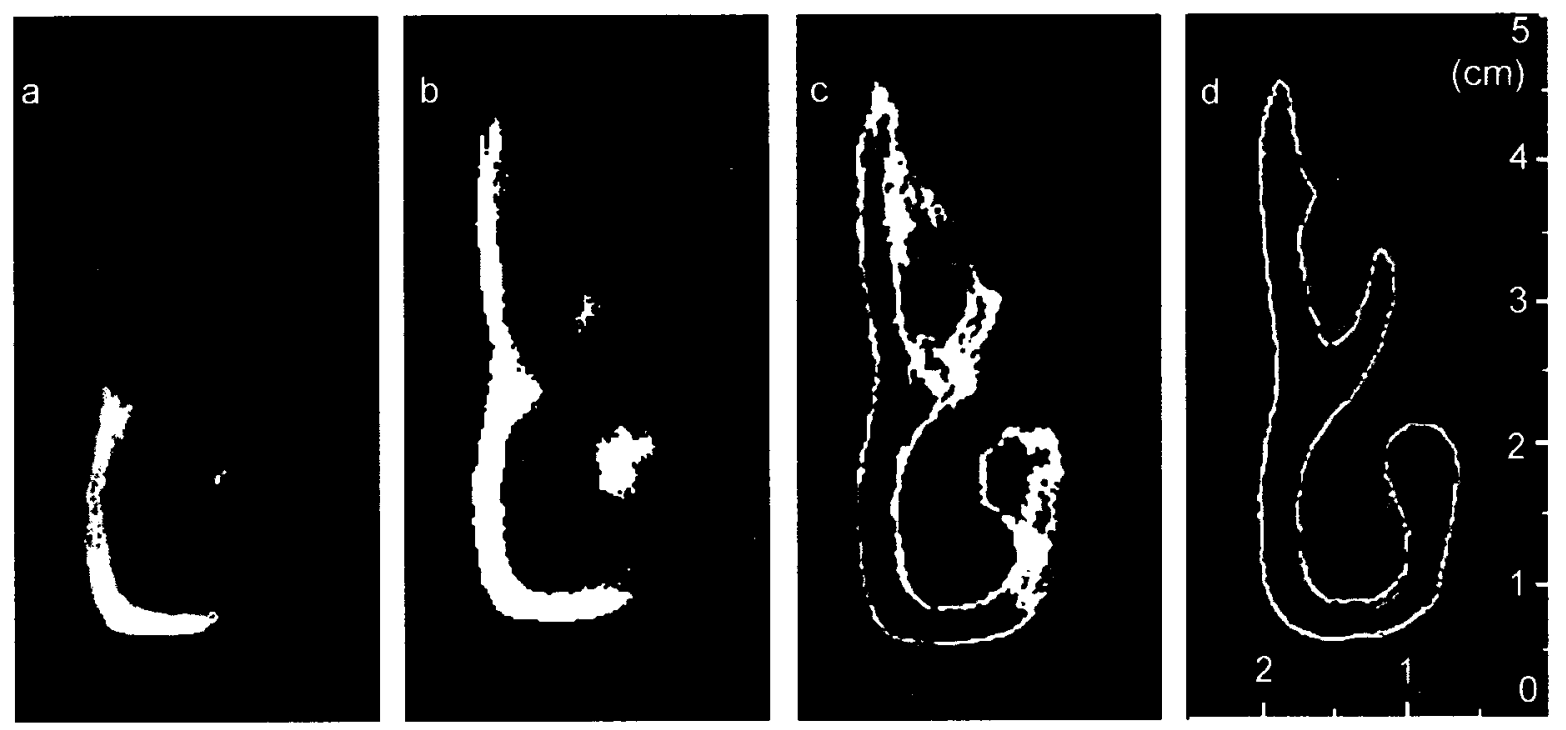

Figure 3.10 Example coronal plane images at $X=30 \mathrm{~mm}$ obtained during processing to determine the characteristic cross-sectional geometry. a) ensemble averaged grayscale image; b) binarized $50 \%$ threshold ("median") image; c) median image in red overlaid on top of "median area" image in yellow; $d$ ) final boundaries of cavity at current $\mathrm{X}$-position in yellow, at next upstream position in green, at next downstream position in red, overlaid on median image in blue

(i) All cross-sectional images at each $\mathrm{X}$-location were classified as one group, and then were binarized (intensities of pixels located within the boundary were set to unity while pixels outside the boundary were set to zero) and summed (intensities at corresponding locations in all of the images were added up pixel by pixel). Thus, a single, ensemble averaged grayscale image for each of the $56 \mathrm{X}$-locations in the model was generated. Figure 3.10.a shows the sample averaged grayscale image for $X=30 \mathrm{~mm}$.

(ii) The second step was generating a "median" image. The "median" image is the pixel by pixel collection of all points that fell within at least $50 \%$ of the nasal cavities in the ensemble averaged grayscale images created in step (i). To accomplish this goal, a 50\% threshold was defined as a threshold value equal to half of the total number of available images, and then was applied to produce a binary image where pixels with value 1 fell within at least $50 \%$ of cavities and pixels with value 0 did not. A sample median image 
for $\mathrm{X}=30 \mathrm{~mm}$ is shown in Figure 3.10.b. The image in Figure 3.10.b is jagged and discontinuous because of the significant geometric variability at this location. This example represents the worst case of the cross-sections; at other locations there was less variability, and the median image was quite smooth.

(iii) Apart from the median image, a separate "median-area" image was also generated at each $\mathrm{X}$ location using a different threshold value. This "auto-threshold" value was iteratively chosen so that that the cross-sectional area in the median-area image would match the median cross-sectional area calculated from up to 60 available images for that location. As shown in Figure 3.10.c, the "median" image from step (ii) is in red, and is over-laid on the "median-area" image in yellow for X=30 mm. It should be noted that the characteristic shape of the nasal airway at this location is much more apparent.

(iv) Using the superimposed images it was possible to make minor manual edits to the shape of the cross-sectional image while constraining the area to match the median crosssectional area of all available subject images. As shown in Figure 3.10.d, to further aid the final editing process, the canny edge detection algorithm (Canny, 1986) was used to calculate and overlay the contours of the nasal cavity geometry from adjacent crosssections. As shown in Figure 3.10d, the working contour at $\mathrm{X}=30 \mathrm{~mm}$ is shown in yellow while the adjacent upstream contour at $\mathrm{X}=28 \mathrm{~mm}$ is shown in green and the adjacent downstream contour at $\mathrm{X}=32 \mathrm{~mm}$ is shown in red. These contours are overlaid on the median-area image which is shown in blue. By several iterative edits and adjustments, the final contour shapes at each cross-section are obtained.

The sample images shown in Figure 3.10 are from the most geometrically variable region of the nasal cavity and hence represent the most challenging $\mathrm{X}$-position for 
determining a characteristic, median geometry. In general, only very minor edits - if any - were required at most X-locations. All sets of images for each of the $56 \mathrm{X}$-positions along the nasal cavity were processed using the procedure described previously, and then 56 cross-sectional shapes for the standardized human nasal cavity were obtained, which are shown in Figure 3.11.

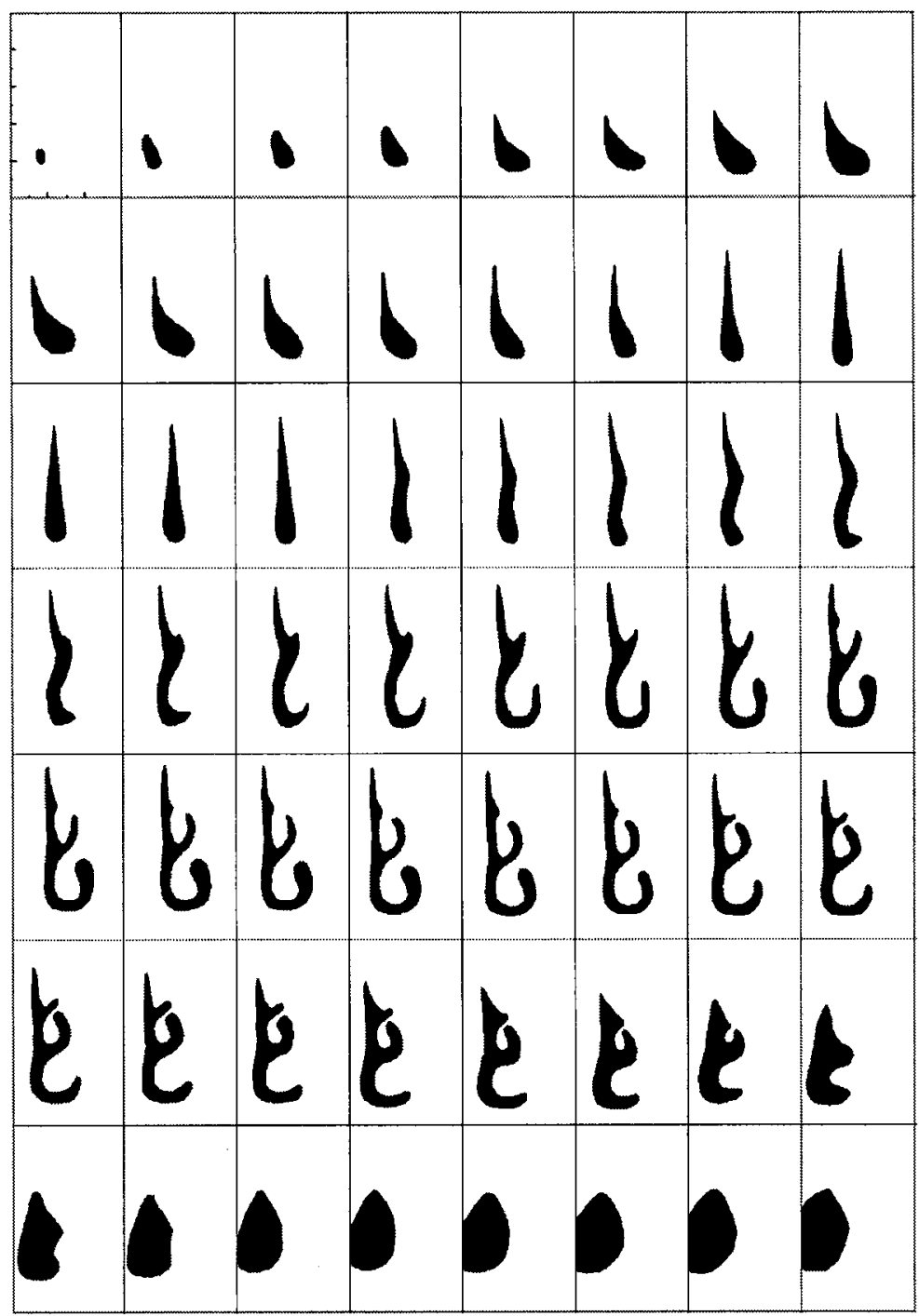

Figure 3.11 56 cross-sectional shapes for the standardized human nasal cavity 


\subsubsection{Determine the shape of nostril and outlet of the standardized nasal cavity}

As previously mentioned, at the nostril entry plane, where there were less data available, the averaged physical descriptors (e.g. major and minor axis lengths in the nostril entry plane and angle between the major axes) were calculated from the measured data (shown in Table 3.3) and supplemented with the measured results of Kesavanathan and Swift (1998). Table 3.4 gives the combined averaged physical descriptors (current data merged with data from Kesavanathan and Swift, 1998) at the nostril entry plane, which were used to determine the shape of the inlet plane of the standardized nasal cavity model.

Table 3.4 Averaged dimensions of the nostril inlet plane (merged raw data from Table 3.3 and from Kesavanathan and Swift (1998)) compared with final values for the Carleton-Civic Standardized Model

$\begin{array}{cccc} & \text { Mean } \pm \text { std } & \text { Range } & \begin{array}{c}\text { Standardized } \\ \text { Model }\end{array} \\ \text { Length of major axis } & 1.76 \pm 0.43 \mathrm{~cm} & 1.18-2.64 \mathrm{~cm} & 1.77 \\ \text { Width of minor axis } & 0.72 \pm 0.12 \mathrm{~cm} & 0.50 \mathrm{~cm}-0.97 \mathrm{~cm} & 0.72 \\ \text { Nostril Angle } & 53.00 \pm 16.61^{\circ} & 17.5^{\circ}-70.1^{\circ} & 67.7^{\circ}\end{array}$

In the posterior region of the cavity, the two separate airways fuse into a single passageway at the choana, which spans the symmetry plane. Thus, as shown in Figure 3.7 , the number of available cross-sectional images suddenly drops from 60 to 30 at the merge point. However, since the goal of this work was to develop a single-sided nasal cavity model, and to remain consistent with the procedure described above, the crosssectional images past the choana were split and mirrored about the symmetry plane. In the final, standard cavity model detailed below, only half of the airway (patient's right 
side) is shown. Thus, if the final model were copied and mirrored, a full two-sided nasal cavity could easily be produced.

\subsubsection{Construction of the final Carleton-Civic standardized nasal model}

Similar to the generation of coarse models, all of the 56 final cross-sectional images were imported into 3D-DOCTOR software (Able Software Corp., Lexington, MA, USA) to generate a coarse standardized nasal mode. This coarse standardized model was imported into ICEM (Ansys Inc., Canonsburg, PA, USA) and was smoothed using the same procedure described in section 3.1.3.4 to create the final, "Carleton-Civic" standardized nasal model. Figure 3.12 shows three views of the final standardized human nasal cavity model.
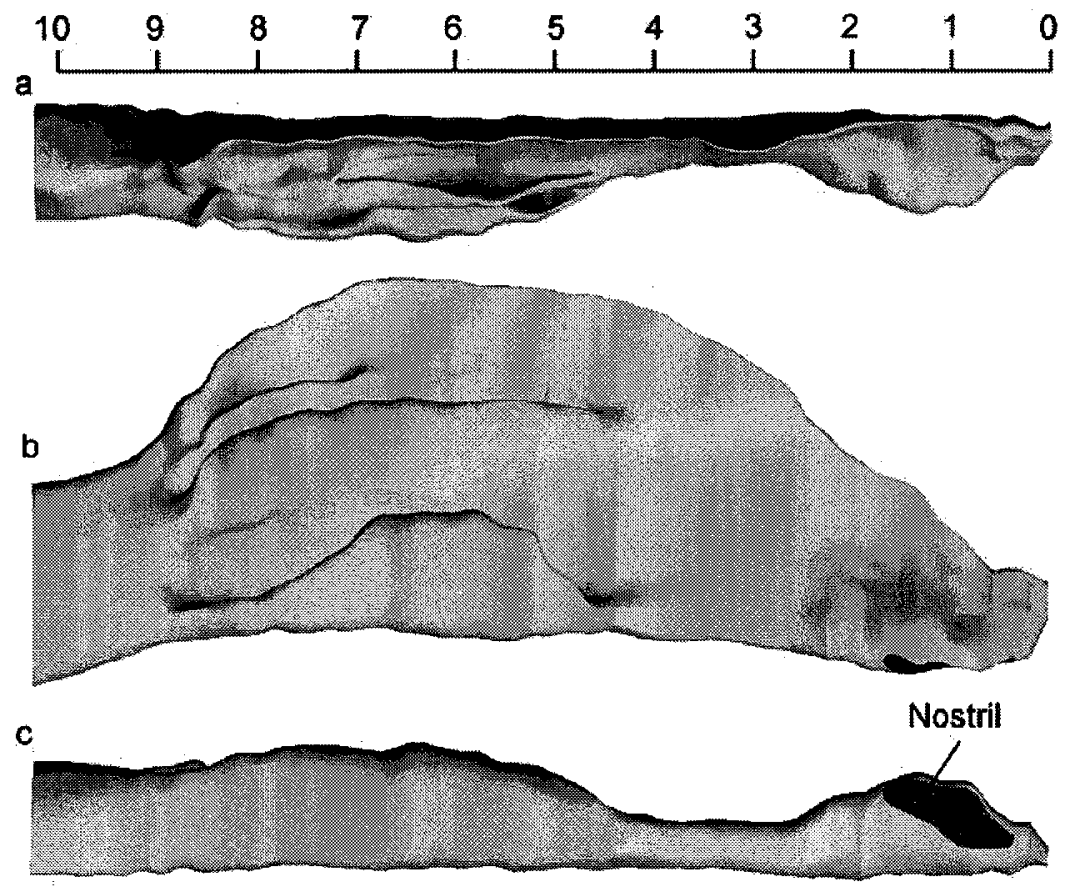

Figure 3.12 Final standardized nasal cavity model. a) Top view, b) Side view; c) Bottom view 


\subsection{Analysis of the Carleton-Civic standardized nasal model}

Prior to using the standardized nasal model to perform experimental and numerical simulations, it is necessary to analyze the model to make sure it can demonstrate the complex geometric features of the nasal passageway as well as a median size and flow cross-sectional area of human nasal cavity.

Figure 3.13 shows the cross-sectional area (CSA) of the standardized nasal model as a function of the distance from the maxillary spine landmark (which is used here as the reference point), compared with the local, median, and averaged CSAs of all subjects used in this research.

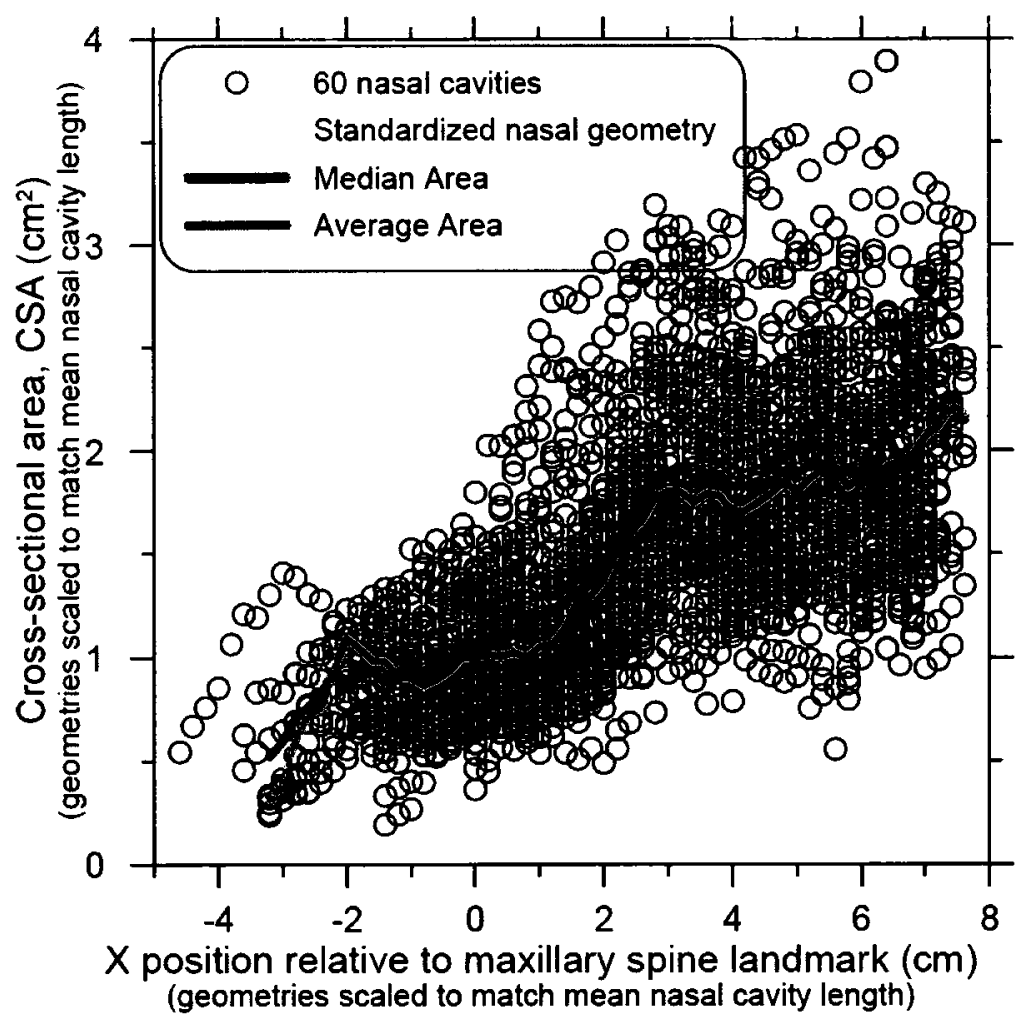

Figure 3.13 Cross-sectional area of the Carleton-Civic standardized nasal model (green curve) compared with individual (dots), median (red curve), and average

(blue curve) cross-sectional areas from all available subjects 
It can be seen from Figure 3.13 that the values of CSA among the 60 nasal cavities vary significantly, which indicates significant variability throughout the 60 individual nasal cavities. However, the CSA of the new standardized geometry very closely tracks the median value of the CSA from all subject cavities, especially in the complex turbinate region. There is a slight deviation in the anterior nostril region, approximately $2 \mathrm{~cm}$ upstream of the maxillary spine; however, the difference is insignificant relative to the notable variability among all models and is negligible when comparing it with the 14 complete nasal cavity models (see Figure 3.14).

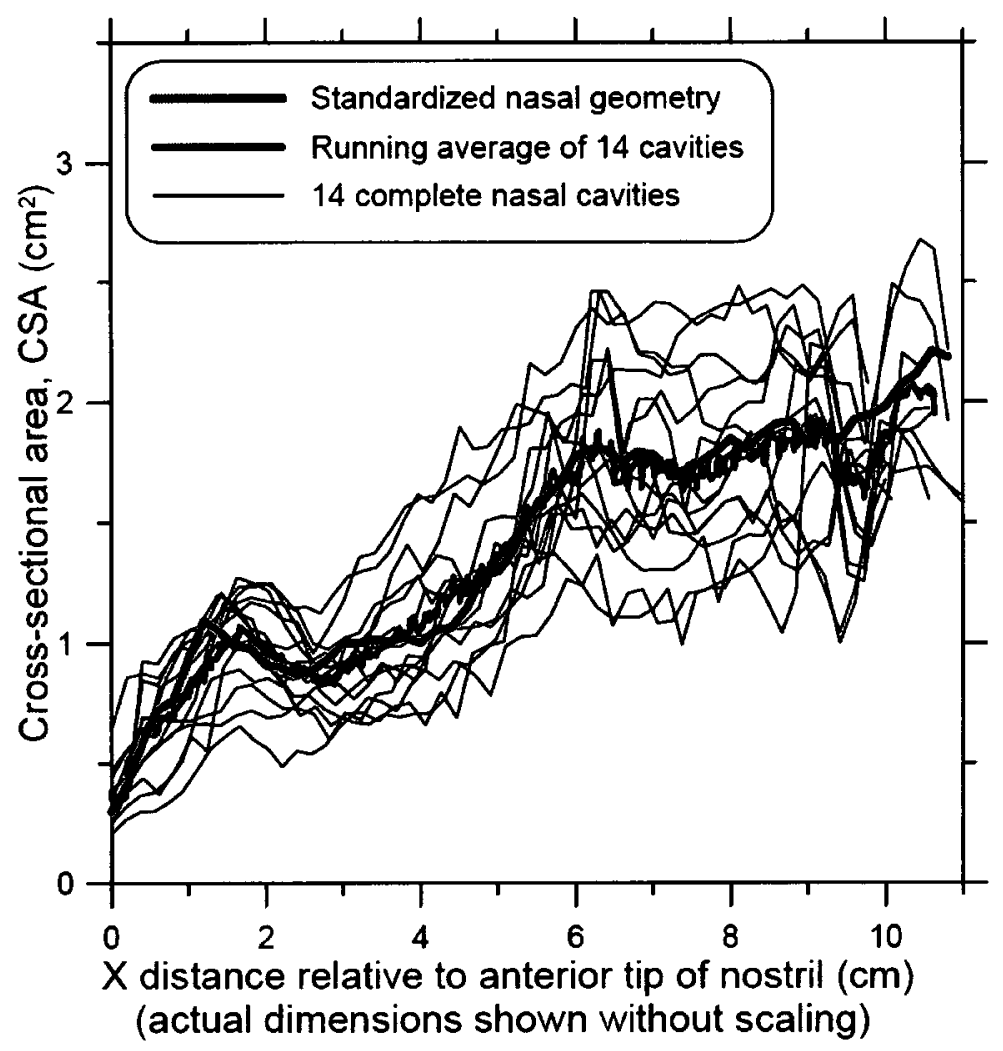

Figure 3.14 Cross-sectional areas of the standardized nasal model (green curve) and 14 complete nasal passages (black curve). Running average of the 14 complete curves is shown for comparison in blue 
Figure 3.14 shows flow cross-sectional areas of the standardized nasal cavity compared with the data of the available 14 complete nasal passages, which allows a closer examination of the CSAs in the nostril region. Data are plotted as a function of distance relative to the tip of the nostril and without any geometric scaling to permit a more critical comparison.

The minimum CSA (MCA) of the nasal models occurs in a region $20-35 \mathrm{~mm}$ from the tip of nostril; the corresponding airway narrowing is called the nasal valve. For the standardized model, the MCA occurs at a location $24 \mathrm{~mm}$ from the nostril, which agrees very well with the averaged curves of 14 cavities. The CSA then increases as expected in the region of the turbinates $(35-75 \mathrm{~mm}$ from the nostril). The data shown in Figure 3.14 indicate that the standardized model effectively captures key geometrical features of the nasal cavity. The axial position of the local maximum in CSA for the standardized model is upstream of the running average local maximum CSA for the 14 complete cavities (dimensionless distance relative to the tip of the nostril equal to 0.12 vs. 0.18 ). This deviation comes from the fact that the anterior part of the standardized model was generated using available data from all geometries, not just that from the 14 complete nasal cavities.

Figure 3.15 shows the CSA of the standardized nasal model as a function of the distance from the nostril, compared with available CSA data extracted from previous published literature, including CT scan derived data (Min et al. (1995), Terheyden et al. (2000) and Kelly et al. (2000)) and MRI derived data (Cheng Y.S. et al. (1996) and Corey et al. (1997)). 
In Figure 3.15, data are plotted as X-position relative to the tip of the nostril using coordinate data as reported. It is important to note that some alignment bias (such as the position of the head of subjects when the CT scans or the MRI data were obtained) would be expected among data in the literature in the absence of a common and rigorous alignment protocol. Because of the generally elongated shape of the nasal cavity, relative misalignment about the $\mathrm{Y}$ or $\mathrm{Z}$ axes should lead to higher estimates of CSA.

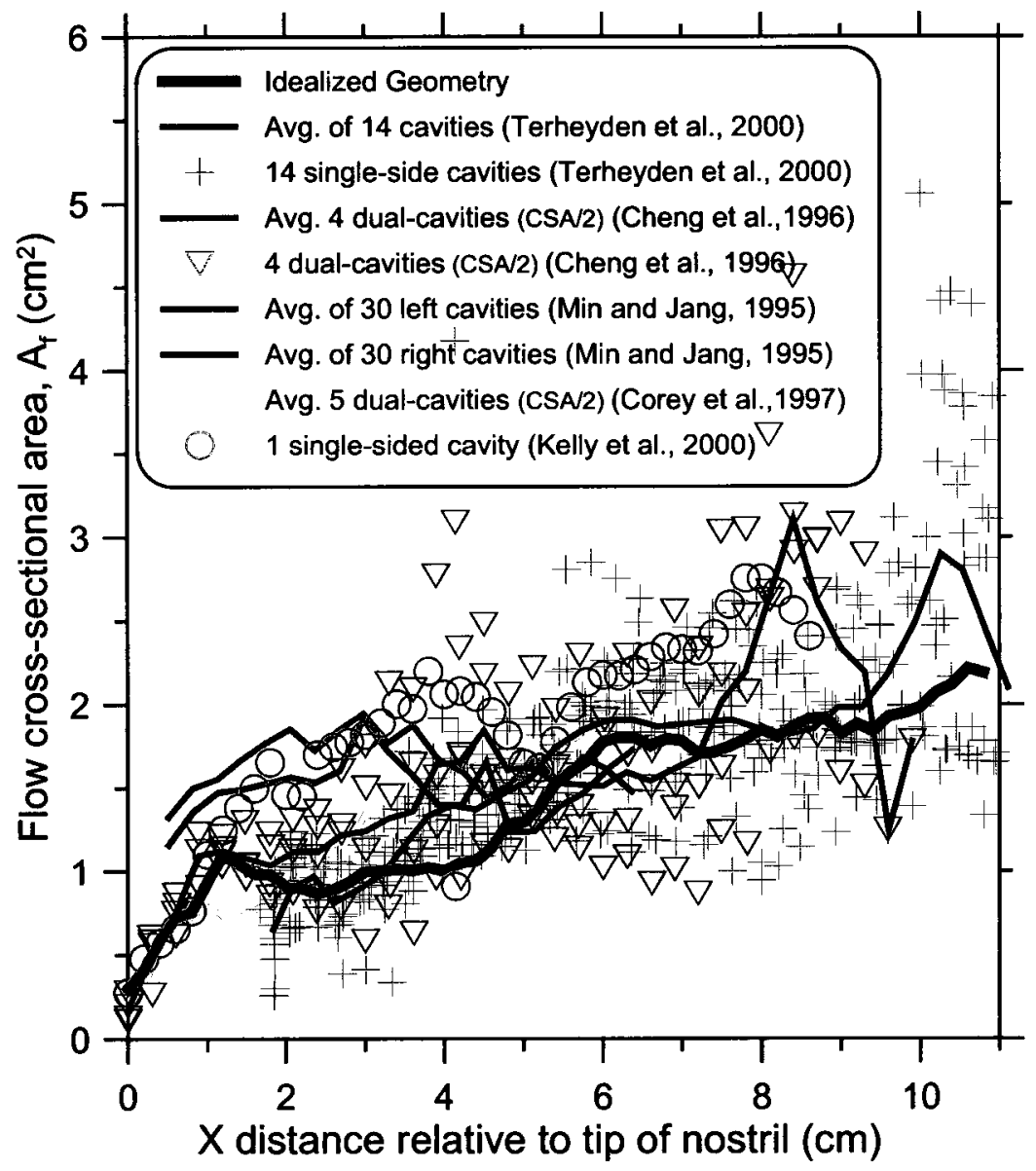

Figure 3.15 Comparison of cross-sectional areas of the Carleton-Civic standardized nasal model (green curve) with published CT scan derived data from Min and Jang (1995) (orange and red curves), Terheyden et al. (2000) (crosses / blue curve) and Kelly et al. (2000) (circles); and MRI derived data from Cheng Y.S. et al. (1996) (inverted triangles / purple curve) and Corey et al. (1997) (yellow curve) 
Similar to the data of the present 60 nasal models, there was significant dispersion in the individual geometry data of previous researchers; however, the standardized model agrees very well with all data at the anterior tip of the nasal passage, and there is a generally consistent trend among averaged data of multiple geometries. In particular, the standardized model CSA characteristic correlates well with the averaged data of 4 cavities from Cheng et al. (1996) (where the reported CSA for the full two-sided cavities have been halved for comparison) and 12 single-sided cavities from Terheyden et al. (2000). On careful observation, the standardized model is on the lower side of the dispersed CSA values in Figure 3.15. It is not necessarily unexpected and can be explained because the current data is slightly biased toward female subjects $(13 \mathrm{M} / 17 \mathrm{~F})$ and the cited data from the literature is heavily biased toward male subjects $(33 \mathrm{M} / 13 \mathrm{~F})$. The data from Min and Jang (1995), which reflect an identical sample size to the current study, do not seem to correlate as well with all of the other data. Although data from Min et al. (1995) span a narrower range, an overall flatter trend in CSA with distance is apparent.

Total volume and surface area of our standardized model (from tip of nostril to choana, single passage) were also measured using the same method explained in the section 3.3.3. Total volume of the standardized model was $13.19 \mathrm{~cm}^{3}$ and total surface area was $90.69 \mathrm{~cm}^{2}$. Guilmette et al. (1997) measured values between 7.37 and 16.91 for volume (average $8.9 \mathrm{~cm}^{3}$ ) and 75.24 and 124.47 (average $91.65 \mathrm{~cm}^{2}$ ) for surface area. The surface area of the standardized model agrees with the results of Guilmette et al. very well, while the value of total volume is bigger than the averaged value reported by Guilmette et al. It is conjectured that the difference is due mainly to the highly complex 
nasal geometry and to differences in the definition of the beginning and end of the nasal cavity. Guilmette et al. calculated the surface area and volume from the anterior nostril to the posterior end of the nasal airway prior to the nasopharynx.

Based on the above discussion, it can be concluded that the methodology for aligning the coarse nasal models and producing median cross-sectional images has been successfully demonstrated through the production of a standardized nasal geometry. This geometry can be used as a standardized human nasal cavity model because it respects and maintains complex characteristic features of the nasal passageway, while tracking a median size and flow cross-sectional area. 


\section{Chapter 4: Methodology of experimental measurement of particle deposition in human nasal cavity}

\subsection{Construction of physical nasal model}

Taking into account the complex flow passage of the human nasal cavity, the rapid prototyping technique was used to construct a physical nasal cavity model for the present particle deposition experiment. The physical nasal model can be constructed using two different methods: one method directly uses the rapid prototyping technique, and the other method uses model casting from the prototype.

\subsubsection{Rapid prototype technique}

Rapid prototyping is the automatic construction of physical objects using solid freeform fabrication. In brief, rapid prototyping takes virtual designs (from computer aided design (CAD) or from animation modeling software), transforms them into cross sections, still virtual, and then creates each cross section in physical space, one after the next until the model is finished.

The first techniques for rapid prototyping became available in the 1980 s and were used to produce models and prototype parts. Today, they are used for a much wider range of applications and are even used to manufacture production quality parts in relatively small numbers. Some sculptors use the technology to produce complex shapes for fine art exhibitions. 
It offers advantages in many applications, compared to classical subtractive fabrication methods such as milling or turning:

(1) Objects can be formed with any geometric complexity or intricacy without the need for elaborate machine setup or final assembly;

(2) Objects can be created in a relatively short time. Of course, the word "rapid" is relative: construction of a model with contemporary machines typically takes 3 to 72 hours, depending on machine type and model size. Used in micro technologies "rapid" is correct. The products created are ready very quickly, and the machines can build the parts in parallel;

(3) Objects can be made from multiple materials or from composites, or materials can even be varied in a controlled fashion at any location in an object;

(4) Additive fabrication systems reduce the construction of complex objects to a manageable straightforward and relatively fast process;

(5) In essence, these machines are three-dimensional printers with a range of accuracy.

These advantages and properties have resulted in the wide use of this method as a way to reduce time in manufacturing and to create model parts used in laboratory research.

\subsubsection{Creation of physical nasal model using RP machine}

Full geometric details of the standardized idealized human nasal cavity model, as described in chapter 3, were translated to STL format, which approximates the surfaces 
of a solid model with triangles and describes the negative flow passage of the standardized nasal cavity model in the present study. The STL file format has become the Rapid Prototyping industry's standard data transmission format, and almost all of today's CAD systems are capable of producing an STL file.

The Department of Mechanical and Aerospace Engineering at Carleton University has two prototyping machines: ZCorp Z400 (Z400.Z Corporation, Somerville, Mass., USA) and 1200es Dimension 3D Printer (Stratasys, Inc., Eden Prairie, USA). The Dimension creates solid models out of ABS plastic. The Z400 is capable of generating solid and water-soluble models using a plaster powder and a binder. For this present application, the physical standardized idealized human nasal cavity model was produced using Dimension. The minimum layer thickness of the Dimension 3D printer was $0.25 \mathrm{~mm}$ (0.010 inches).

To measure regional as well as total particle deposition efficiencies in the standardized human nasal cavity, the replica was separated into three sections using a similar definition to the one described in section 3.3.1 :

(i) the anterior/nostril region (32 $\mathrm{mm}$ long, from anterior tip of the nostril to anterior maxillary spine landmark);

(ii) the middle/nasal airway region (64 mm long, from the anterior maxillary spine landmark to the choana);

(iii) the posterior region (10 $\mathrm{mm}$ long, corresponding to the region posterior to the choana known as the nasopharynx). 
In addition, anterior and posterior connection pieces were generated to permit connection of the nasal replica to the aerosol generator and outlet pipeline for experiments. Figure 4.1 gives the illustration of the individual parts of Carleton-Civic standardized nasal cavity used for deposition experiments in the ICEM-CAD environment with transparent outer surfaces.

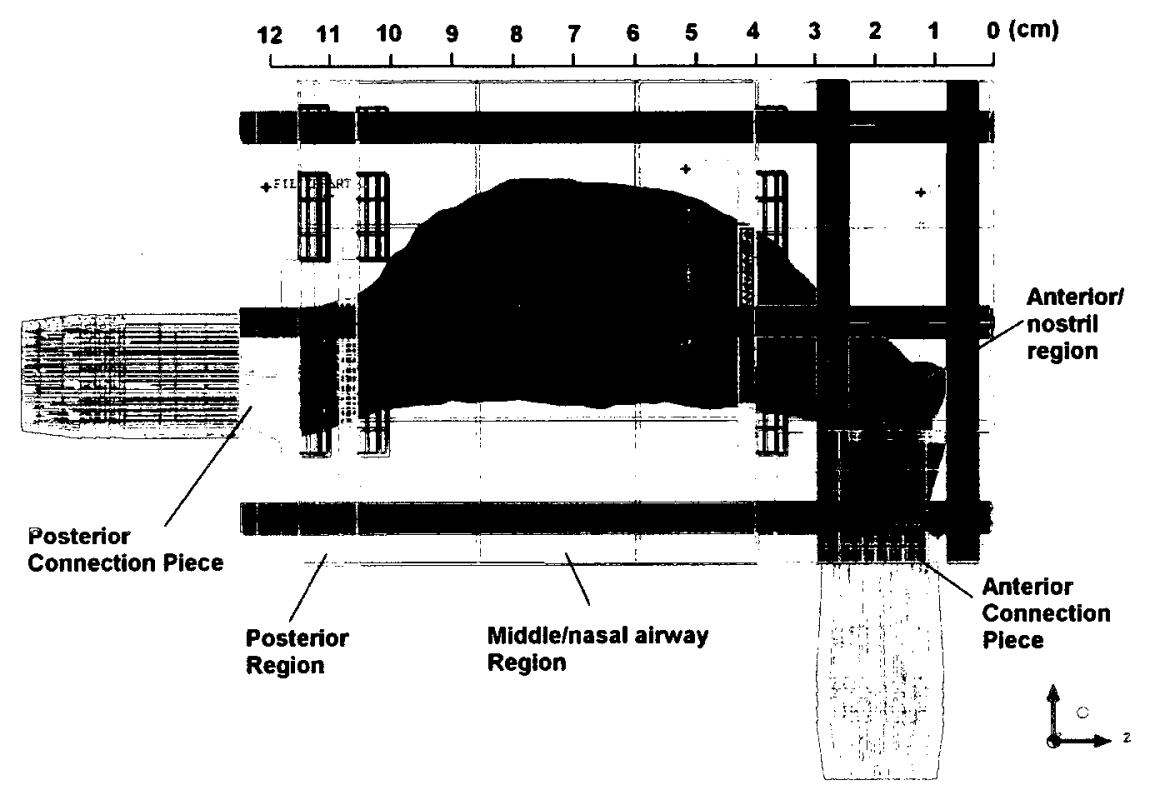

Figure 4.1 Carleton-Civic standardized nasal cavity used for deposition experiments in the ICEM-CAD environment with transparent outer surfaces

Figure 4.2 shows the three regions of the physical model produced using Dimension, along with the two connection pieces. Once all parts of the replica were assembled, they were enclosed in a negative nasal cavity. 


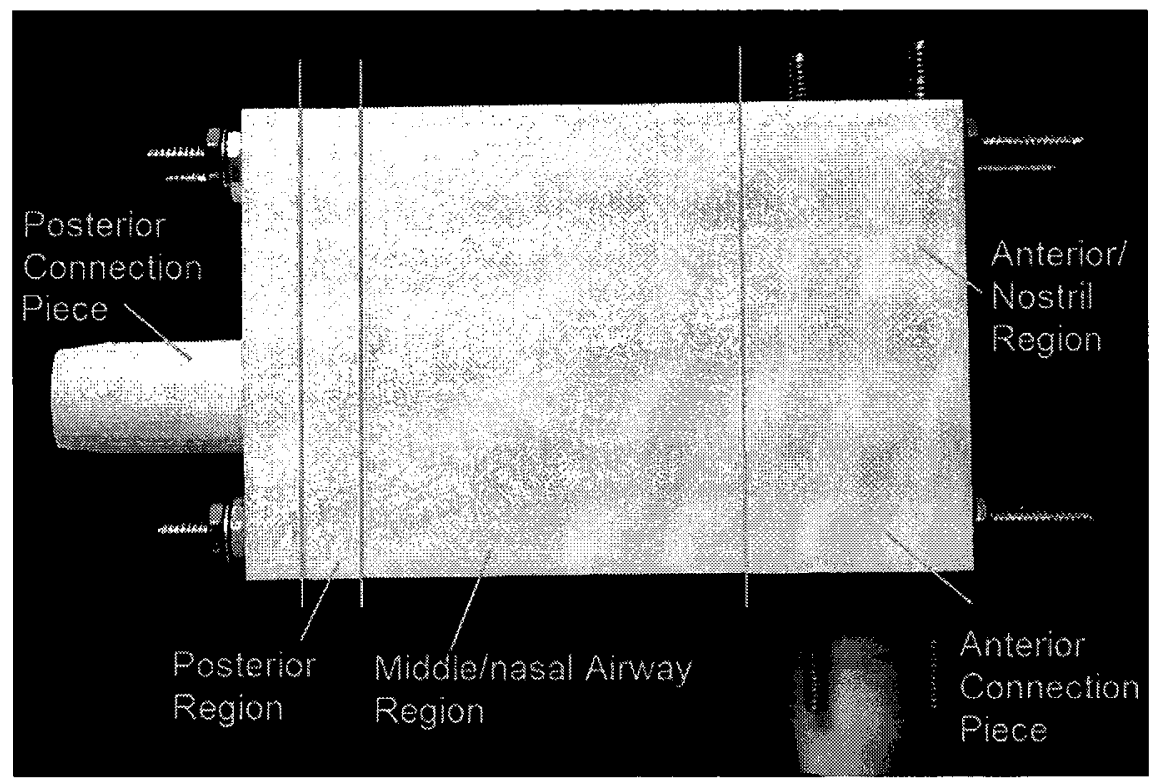

Figure 4.2 Carleton-Civic standardized nasal cavity used for deposition experiments: three regions of the physical model along with the two connection pieces

\subsection{Experimental apparatus and procedures}

The deposition experimental setup is shown in Figure 4.3 The main parts of the experimental setup are as follows:

1) Aerosol generator (Model 3450 Vibrating Orifice Aerosol Generator, TSI Incorporated, Shoreview, USA): used to generate monodisperse aerosol from Vitamin E.

2) Mixing chamber: helped to ensure complete mixing of the aerosol particles with diluted air in order to reduce the concentration of the aerosol; also allowed the flow rate to be controlled by the introduction of secondary air.

3) Particle Size Distribution Analyzer (PSD 3603 Particle Size Distribution Analyzer, TSI Incorporated, Shoreview, USA): used to monitor the aerosol particles sizes and monodispersity. 
4) Nasal Cavity Model.

5) Filter (Marquest Respirgard- II 303 Bacterial filter manufactured by Vital Signs Inc.): used in series at the outlet of the test nasal model to "keep" the oil particles out of nasal passage.

6) Vacuum pump, flow meter, pressure gauge, adjustment valve: used to set, monitor and regulate the steady inhalation flow rate. The steady inhalation volume flow rates of 60 and $90 \mathrm{~L} / \mathrm{min}$ were used in the experiments.

7) Solution concentration analyzer (Agilent 8453 UV-visible spectroscopy System, Aglient Technologies, Inc., Waldbronm, USA): used to obtain concentration of Vitamin E from the solution obtained from the washing of nasal model and downstream part.

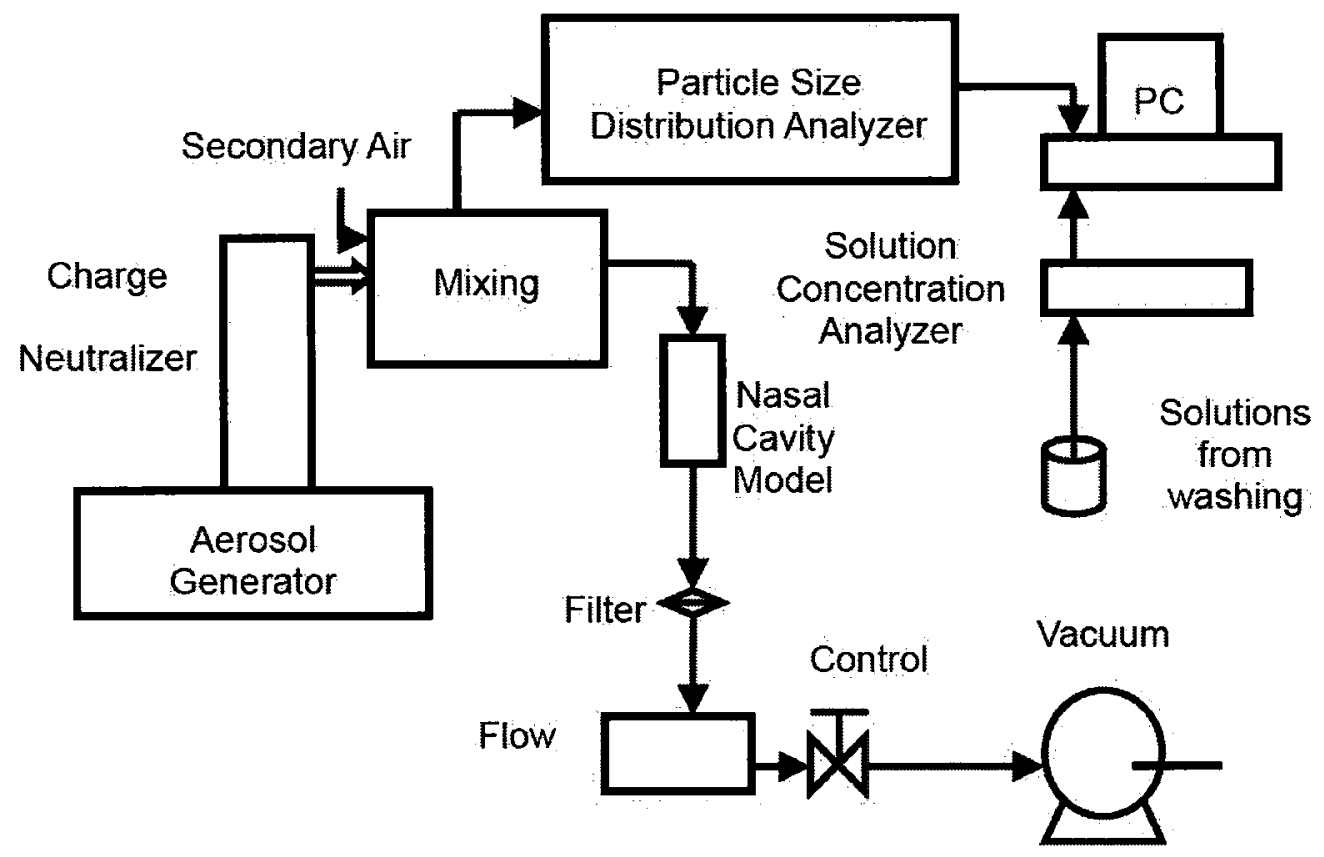

Figure 4.3 Schematic of experimental apparatus 


\subsubsection{Aerosol generation}

\subsubsection{The aerosol generator}

Liquid material always produces particles with spherical shape and of uniform density because of surface tension (Wen, 1996), thus both geometric diameter and aerodynamic diameter of the particles can be calculated accurately. Aerosols produced with liquid material are highly-monodisperse, with a typical standard deviation better than one percent.

Monodisperse aerosols (i.e., particles with a very narrow size distribution) were generated from Vitamin E oil using a controlled Vibrating Orifice Aerosol Generator (VOAG) (Model 3450, TSI Incorporated, Shoreview, USA), which can produce a highquality monodisperse aerosol with specific particle size and concentration from any material in solution form. Even though a wide variety of solutions can be used with the Model 3450 VOAG (as shown in Figure 4.4), the monodisperse aerosol generated from Vitamin E was used in this experiment. It dissolved in Isopropyl alcohol, which was adopted as the solvent in present experiment because it has several advantages such as low viscosity and low vapour pressure, easy to work with, and easily evaporates in the drying column.

The jet of liquid was created by pumping the vitamin $E$ (brand Equate ${ }^{\circledR}$ ) solution out of a syringe with a stepper motor, into tubing that directs the solution to the orifice. A constant volumetric flow rate was generated by the jet of liquid, and as it passed through the small orifice (with diameters of 5,10 or $20 \mu \mathrm{m}$ ) it broke into uniform size droplets due to the vibration of the orifice. The orifice vibrated at the same frequency as the 
frequency generator because it was in mechanical contact with a piezo-electric ceramic, which was electrically connected to a frequency generator. The frequency of vibration of the orifice can be adjusted from $1 \mathrm{kHz}$ to $1 \mathrm{MHz}$.

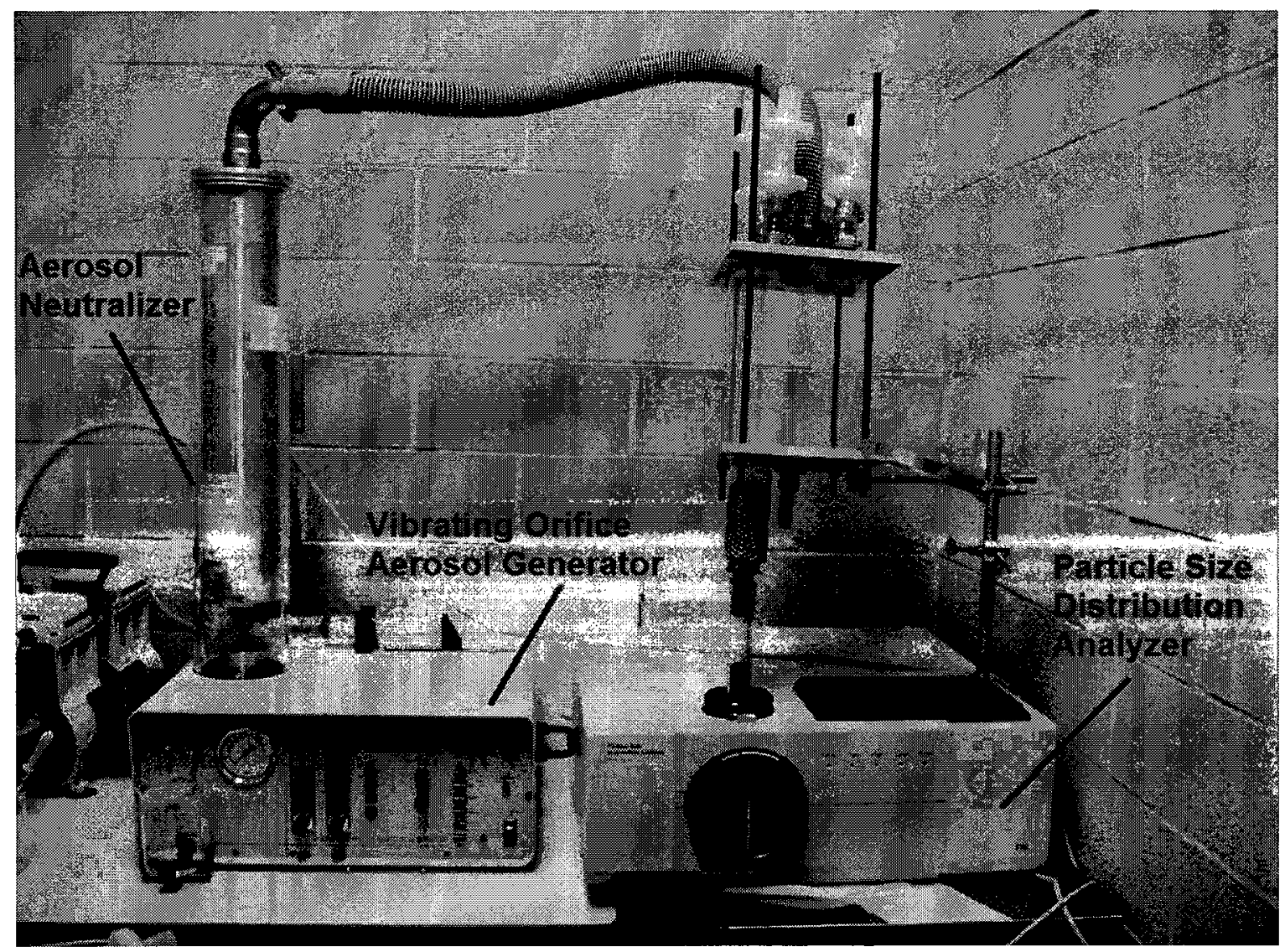

Figure 4.4 TIS Model 3450 Vibrating Orifice Aerosol Generator (left, including the TSI Model 3054 Aerosol Neutralizer) and TIS 3603 Particle Size Distribution Analyzer (right)

\subsubsection{Aerosol generation principle}

When the liquid feed rate and orifice vibration frequency are chosen, the final particle size is determined by the concentration of vitamin $E$ in the isopropyl solution. A run speed of $8.2 \times 10^{-4} \mathrm{~cm} / \mathrm{s}$ is recommended for a $20 \mu \mathrm{m}$ orifice with a $20 \mathrm{~cm}^{3}$ syringe. But 
in actual operation, this value can be adjusted to obtain a better particle size distribution. The difficulty in operating the aerosol generator is determining the optimum orifice vibration frequency for producing monodisperse droplets.

For a particular setting (combination of liquid feed rate and orifice vibration frequency) the particle size can be determined by following equation (TSI, 2002):

$$
d_{p}=\left(\frac{6 Q_{s}(C+I)}{\pi f}\right)^{1 / 3}
$$

where $d_{p}$ is the calculated particle diameter; $Q_{s}$ is the volume flow rate of the jet ( $=5.44 \times 10^{-9} \mathrm{~m}^{3} / \mathrm{s}$ for the syringe pump setting of $9.9 \times 10^{-4} \mathrm{~cm}^{-4} / \mathrm{s} ; 1 \%$ bias limit); $f$ is the vibration frequency of the frequency generator ( $1 \%$ bias limit); $C$ is the volumetric concentration of the solution of vitamin $\mathrm{E}$ (brand Equate $\left.{ }^{\circledR}\right)$ in alcohol $(C=0.000196 \times$ Absorbance at $285 \mathrm{~nm} ; 2 \%$ bias limit) and $I$ is the residue after evaporation volumetric concentration of the alcohol (40 ppm).

\subsubsection{Operation of aerosol generation}

The steps to operate the Model 3450 include:

(1) Flushing the liquid feed system;

(2) Eliminating Air Bubbles;

(3) Starting the liquid jet;

(4) Tuning the signal generator to the proper operating frequency;

(5) Installing the aerosol neutralizer; 
(6) Adjusting the dispersion and dilution air;

(7) Stopping the liquid jet.

\subsubsection{The charge neutralizer}

Usually, aerosol particles generated using laboratory generators are highly charged, which is undesirable for the researchers because it can affect the deposition size fraction measurements. The level of charge on aerosols can be reduced by means of small bipolar ions. By exposing an aerosol to a source of ionizing radiation, small bipolar ions can be created and a gaseous medium around the particles can be made electrically conductive. The particles can then discharge themselves by capturing ions of the opposite polarity (Liu and Pui, 1974). In present work, the TSI Model 3054 Aerosol Neutralizer (TSI Incorporated, Shoreview, USA) is used, which consists of 10 millicurie of Krypton-85 gas as a radioactive source sealed in a small diameter, thin-walled stainless steel tube placed along the centre of a cylindrical stainless steel container, as shown in Figure 4.4.

The $\beta$ - radiation produced by the radioactive $K r^{85}$ gas can penetrate through the thinwalled stainless steel tube to ionize the gas molecules outside. The outer stainless steel cylinder performs two functions: to confine the $\beta$-radiation produced by the radioactive $K r^{85}$ gas and to provide the necessary neutralization volume for the aerosol.

\subsubsection{The particle size distribution analyzer}

Prior to flowing into nasal cavity model, some of the aerosol is fed into the TSI 3603 Particle Size Distribution Analyzer (PSD) (TSI Incorporated, Shoreview, USA), which is 
shown in Figure 4.4. Section 4.2.1.2 explained that the particle size can be calculated for a particular setting of the aerosol generator (combination of liquid feed rate and orifice vibration frequency). However, the generated size of particles we generated may be different from the calculated value since each orifice is slightly smaller or larger than the nominal diameter due to manufacturing tolerances. Although this does not have direct effect on the final particle size, it does have an effect on the operating frequency range of a particular orifice, and in turn the operating frequency will affect the size of the generated particle. Although the dispersion and dilution air are adjusted for each run, it is impossible to generate absolutely uniform particles at optimum dispersion airflow. Thus, it is indispensable and necessary to measure and analyze the particle size distribution. PSD 3603 Particle Size Distribution Analyzer was used in our present experiments to measure the aerosol particle size, as well as to ensure the aerosol droplets were monodisperse.

The operational principle of the PSD is described in the manual of this device (TSI, 2004). Air with aerosol from aerosol generator was bled from the mixing chamber at flow rate of $7 \mathrm{~L} / \mathrm{min}$ into the PSD. Most of the air (with flow rate of $6 \mathrm{~L} / \mathrm{min}$ ) was diverted and filtered, and then re-directed through a nozzle. The rest of the air containing the aerosol $(1 \mathrm{~L} / \mathrm{min})$ flowed though a smaller inner nozzle, which was installed inside the outer nozzle. The aerosol exited this inner nozzle into the sampling area. Sheath air from the outer nozzle with flow rate of $6 \mathrm{~L} / \mathrm{min}$ surrounded the aerosol, causing the flow from the inner nozzle to form a central region of more uniform flow. The aerosol particles were accelerated to a high velocity in a narrow stream, and they lagged behind due to their inertia. During this acceleration, particles passed individually through a pair 
of overlapping laser beams. Light scattered off the particle from each beam passed through a lens to an avalanche photo-detector, which produced a distinct double-crested electrical pulse. The time between the crests was determined by the drag force on the particle. The air continued into a diffuser and the particles were trapped downstream.

The PSD 3603 is an integrated system for particle size measurement which provides fast, reliable results, and the bias limit of the measurement is 2\%. The PSD 3603 works with the Particle Instrument Manager (PIM) software that provides an intuitive user interface and allows for efficient data analysis and regulatory compliance. When data collection is started, the run properties dialog box opens and Size Graph, Size Table, Cumulative Table and Statistics Table are available to show particle statistics, including size range, geometric mean value and geometric standard deviation. By monitoring the size graph, cumulative table and statistics Ttable, it is not difficult to tell whether the aerosol is monodisperse or not. A sample size distribution plot is shown in Figure 4.5 below.

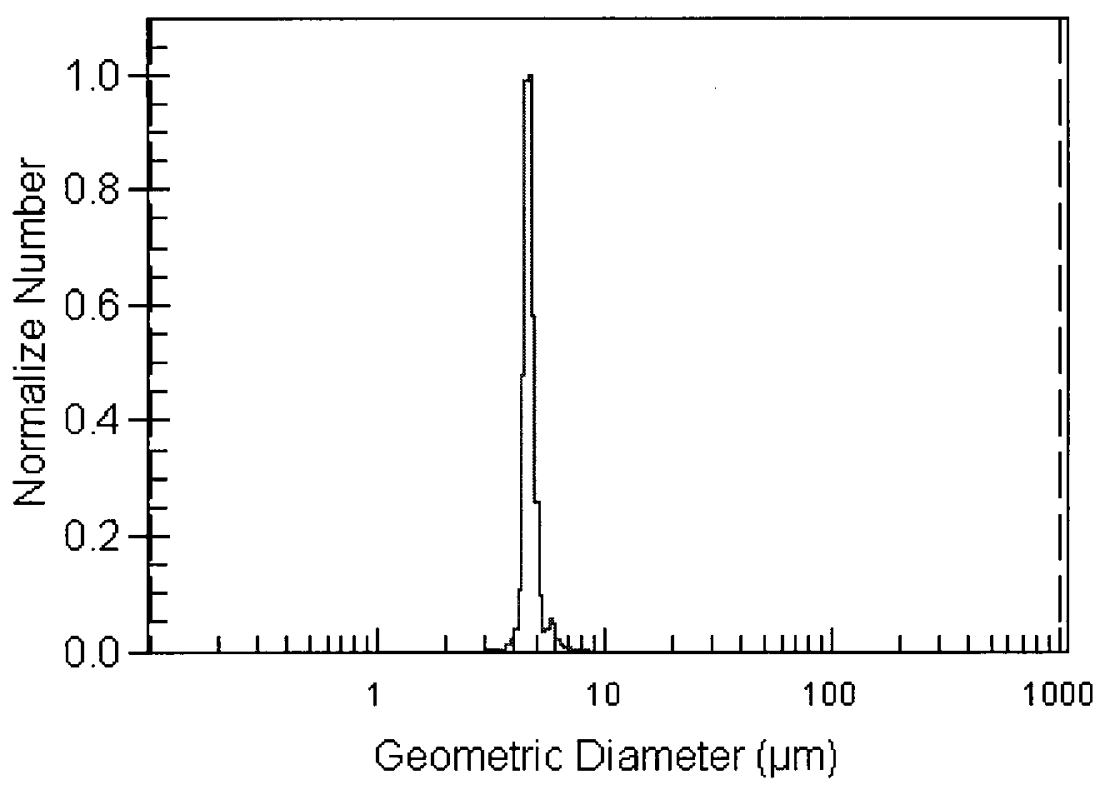

Figure 4.5 Sample particle size graph, $d_{p}=4.62 \mu \mathrm{m}$ 


\subsubsection{Other parts of the flow system}

\subsubsection{The vacuum pump}

A Welch dry vacuum pump Model 2585B (Gardner Denver Welch Vacuum Technology Inc.) was adopted in current measurements to drive the flow. The pump was placed downstream of the mass flow meter and manual control valve. It was capable of pulling about $200 \mathrm{~L} / \mathrm{min}$ through the system, which was sufficient for the present testing flow rates.

\subsubsection{The mass flow meter}

In the present experiment, the flow rate was measured using the Brooks Instrument Model 5863S Thermal mass flow meter. The principle of operation is based on the equipment manual (Brooks Instrument, 2000). Air was diverted through a bypass channel, where it flowed past a heating element. The power supplied to the heating element was known or constant. The temperature difference between upstream and downstream was measured using two sensing elements, one upstream and one downstream. This temperature difference is proportional to the mass flow rate. The two sensing elements were connected in a bridge circuit. The resistance in each element depends on its temperature. Using the bridge circuit, the difference in resistance between upstream and downstream was determined, which gave the temperature difference. A voltage output signal proportional to the mass flow rate was generated and fed into a National Instruments data acquisition card, which was read by the LabView software. 
The maximum flow rate of this flow meter was 150 SLPM (the standard litres per minute of air at a pressure of $101.325 \mathrm{kPa}$ and a temperature of $0{ }^{\circ} \mathrm{C}$ ), which was sufficient to measure the test flow rate used in the present study. The bias error of the flow meter is $0.2 \%$ of the full scale ( 0.3 SLPM) and $0.7 \%$ of the operating range.

\subsubsection{Particle deposition measurement}

Approximately two hours were required to complete the flow portion of the experimental protocol for detecting particle deposition fraction. Data for several inhalation mass flow rates and particle sizes were collected, and thus the time to achieve detectable levels and sufficient deposition for accurate measurement varied. The protocol was repeated three times for each particle size at each flow rate using a solution with the same concentration of particles to decrease precision uncertainty.

After the flow part of the experiment was finished, the nasal model and the filter were detached from experimental setup and the vitamin $\mathrm{E}$ was washed from the three parts of the nasal model and from the filter. Then the amount of vitamin $\mathrm{E}$ was measured with the spectrophotometer and the total deposition fraction was calculated.

\subsubsection{Wash procedure}

To measure the amount of deposited Vitamin E in the nasal cavity, the three parts of the standardized nasal model and filter were washed separately with solvents (methanol, typically $10 \mathrm{~mL}$ ). It was necessary to repeat washing at lease five times for each part of 
nasal model because of its complex geometry. The filter was soaked in a beaker containing a fixed amount of isopropyl alcohol (typically $10 \mathrm{~mL}$, same as used for the parts of nasal cavity model). The beaker contained the filter pad detached from the filter, and the beaker was shaken for several minutes to allow the Vitamin $\mathrm{E}$ to dissolve sufficiently in the methanol.

\subsubsection{The spectrophotometer}

An Agilent 8453 UV-visible Spectroscopy system (Agilent Technologies) shown in Figure 4.6 was used in the present experiment to measure the concentrations of vitamin $\mathrm{E}$ in the isopropyl alcohol solutions obtained from the wash procedure. The principle and description of this instrument is based on the instrument manual (Agilent Technologies, 2003).

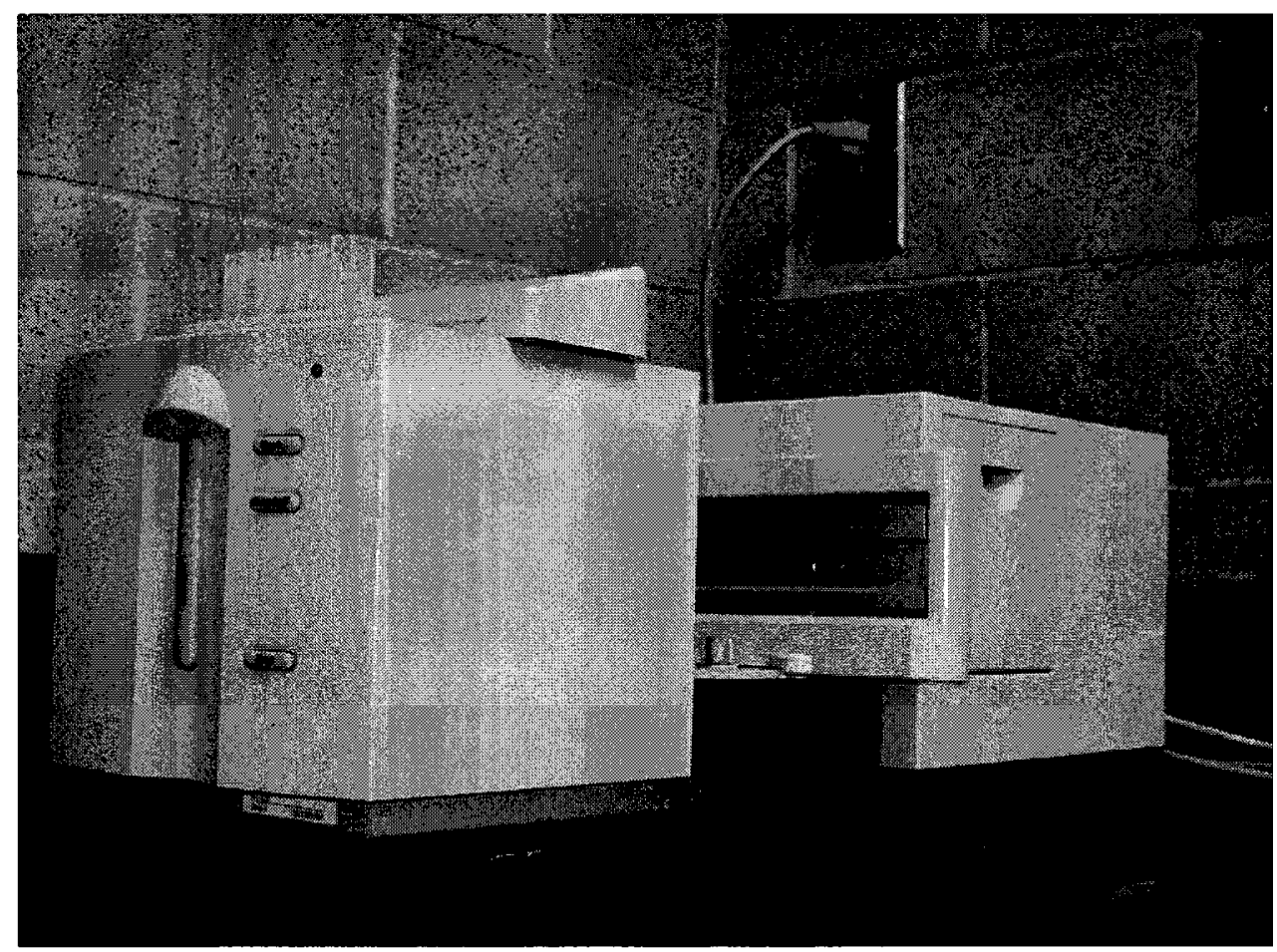

Figure 4.6 Agilent 8453 UV-visible Spectroscopy system 
For one sample, its absorbance peak occurs in the range from $190 \mathrm{~nm}$ to $1100 \mathrm{~nm}$ in $1 \mathrm{~nm}$ increments, and the absorbance $(\mathrm{Ab})$ is defined as:

$$
A b=\log _{10} \text { (Incident light intensity/Transmitted light intensity) }
$$

The absorbance peak of Vitamin E occurs at $285 \mathrm{~nm}$ and spans a wavelength range of $252-300 \mathrm{~nm}$. The absorbance measured at $320 \mathrm{~nm}$ is used as reference and is subtracted from each absorbance measured at $285 \mathrm{~nm}$ to correct for impurities in the solution. The absorbance value used to calculate the particle deposition fraction is the wavelength-weighted average over $252-300 \mathrm{~nm}$.

Any uncertainty in the intensity of the transmitted light causes an uncertainty in the absorbance proportional to $10^{\mathrm{Ab}}$, which grows very quickly when $\mathrm{Ab}$ is greater than 1 . Therefore, if the measured absorbance at $285 \mathrm{~nm}$ was greater than 1.5 , the measurement was not used.

The total particle deposition fraction $(D F)$ can be calculated using Equation 4.3:

$$
D F=\frac{M_{n}}{M_{n}+M_{f}}
$$

where $M_{n}$ and $M_{f}$ are the masses of particles deposited in the nasal model and in the down-stream filter, respectively. Because the same volume of methanol was used to wash the three parts of nasal model and filter, and the absorption is directly proportional to the concentration of a solution according to Beer's Law (Bauman and Poe, 1962), and the total deposition fraction can also be obtained by:

$$
D F=\frac{\sum_{i=1}^{3} \operatorname{Vol}_{n_{i}} A b_{n_{i}}}{\sum_{i=1}^{3} V o l_{n_{i}} A b_{n_{i}}+V o l_{f} A b_{f}}
$$


where, $\operatorname{Vol}_{n}, \mathrm{Vol}_{f}$, and $A b_{n}, A b_{f}$ are the volume and absorbtion of solutions obtained from the washing of nasal model and down-stream filter, respectively.

\subsection{Uncertainty analysis}

True values of experimental results are seldom known and experiments always have errors due to instruments, data acquisition, data reduction, and environmental effects. It is important to determine the uncertainty for risk assessments in experimental set up design and for using data directly or in calibrating and/or validating simulation methods. In this section, uncertainty analysis will be performed based on the ANSI/ASME Measurement Uncertainty Standard (1985).

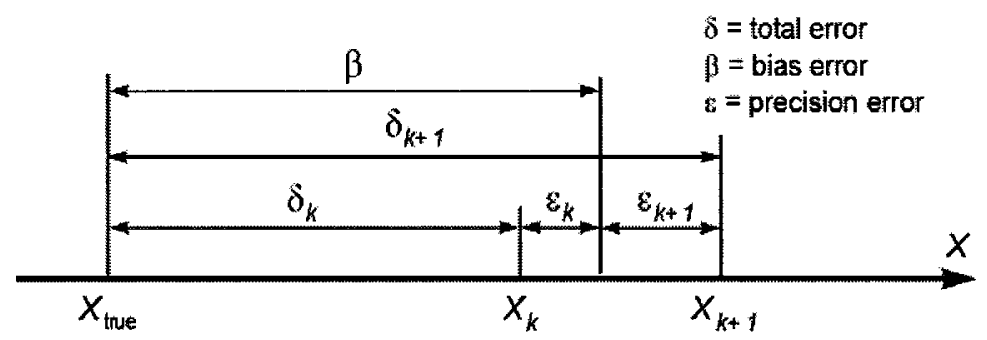

(A) Two readings

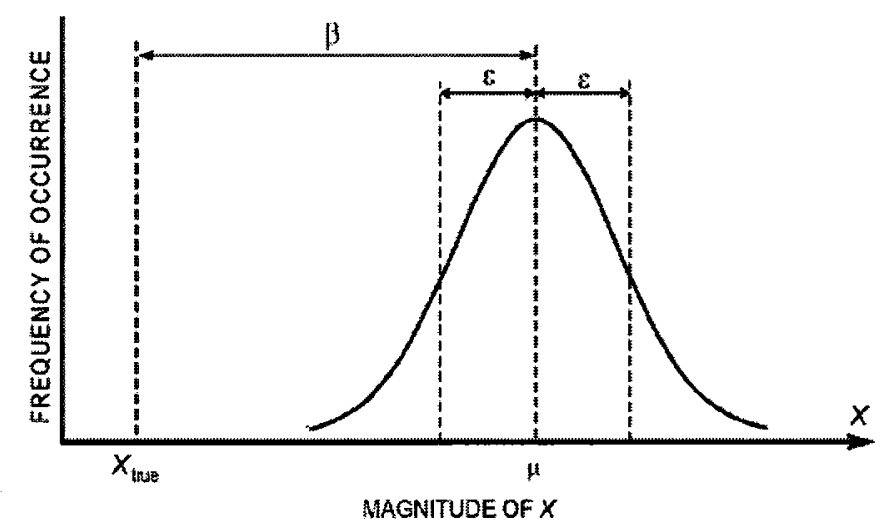

(B) Infinite number of readings

Figure 4.7 Errors in the measurement of a variable $X$ (Coleman \& Steele, 1999) 
As illustrated in Figure 4.7, there are two types of uncertainty measures for data: the propagated bias error, and the precision. Bias errors $(\beta)$ are those due to inability of the equipment to measure true values or the faults in the measuring instruments or in the techniques used in the experiments, thus, they are also called systematic errors. The propagated bias error is the estimate of the influence of all the different sources of bias error on the final measurement. The precision (random) error $(\varepsilon)$ is the scatter in the measurements caused by random errors, which are associated with unpredictable variations in the experiment, or are due to a deficiency in defining the quantity being measured. In the following sections, different types of bias errors and precision errors are discussed, estimated separately and then combined together to get the total error $(\delta)$.

\subsubsection{Bias Error}

Strictly speaking, it is not possible to measure the actual bias error of any particular instrument, so actual bias error must be estimated. The estimated bias error is called the bias limit, and it represents the likely range in either the positive or negative direction that contains the true bias. Thus the bias limit is also called symmetric limit. When there are several different bias limits to be combined (for a function $f\left(x_{1}, x_{2}, \square \square x_{N}\right)$ ), it is assumed that there are as many negative bias errors as positive errors, so they can be roughly combined in quadrature by the root sum of squares formula shown in Equation 4.5. When there are only a few bias limits to be combined, the same equation is used, but it is a less certain estimate, since they might actually combine additively. Propagated bias error for a function $f\left(x_{1}, x_{2}, \square \square x_{N}\right)$ is calculated according to following formula: 


$$
\beta=\sqrt{\beta_{1}^{2}+\beta_{2}^{2}+\square \beta_{N}^{2}}
$$

where, $\beta, \beta_{1}, \beta_{2}, \square, \beta_{N}$ are the propagated bias error, bias limit 1, bias limit $2, \sqcup$, bias limit N. The bias limits can be calculated using Equation 4.6.

$$
B=\frac{\partial f\left(x_{1}, x_{2}, \square, x_{N}\right)}{\partial x_{1}} \beta_{x 1}
$$

As previously stated, in present experimental research, the total particle deposition in nasal cavity, $D F$, is defined as function of inertial parameter, $I P$, that is:

$$
D F=f(I P)=f\left(\rho d_{p}^{2} Q\right)=f\left(d_{a}^{2} Q\right)
$$

where, $\rho\left(\mathrm{kg} / \mathrm{m}^{3}\right)$ is the density of particles, $d_{p}$ (usually in $\mu \mathrm{m}$ ) is the geometric diameter of particles, $d_{a}$ (usually in $\mu \mathrm{m}$ ) is the aerodynamic diameter of particles, $Q(\mathrm{~L} / \mathrm{min}$ ) is the inhalation flow rate. In the following subsections, bias errors due to different factors will be discussed separately.

\subsubsection{Inertial parameter}

The bias limit of the particle density is a function of the vitamin $\mathrm{E}$ used to generate

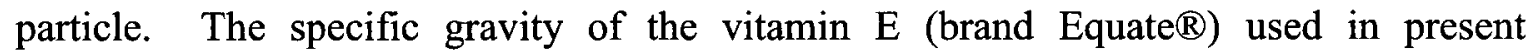
experimental work is $0.92 \pm 0.01$. Thus the bias limit of the particle density can be calculated using following equation:

$$
\frac{\beta_{\rho_{P}}}{\rho_{P}}=\frac{1 \%}{0.92}=1.09 \%
$$


As discussed in the section 4.2.1.2, the particle size was calculated based on the operation of the aerosol generator, thus the bias limit of the geometric diameter of particles can be obtained using equation 4.9 :

$$
\frac{\beta_{d}}{d}=\frac{1}{3} \sqrt{\left(\frac{\beta_{Q_{s}}}{Q_{S}}\right)^{2}+\left(\frac{\beta_{f}}{f}\right)^{2}+\left(\frac{\beta_{C+I}}{C+I}\right)^{2}}
$$

For the aerodynamic diameter of particles, the bias limit of the density of the vitamin E must be included in the calculation:

$$
\frac{\beta_{d_{a}}}{d_{a}}=\frac{1}{3} \sqrt{\left(\frac{\beta_{Q_{s}}}{Q_{S}}\right)^{2}+\left(\frac{\beta_{f}}{f}\right)^{2}+\left(\frac{\beta_{C+I}}{C+I}\right)^{2}+\left(\frac{\beta_{\rho}}{\rho}\right)^{2}}
$$

Prior to being put into the syringe of the aerosol generator (about $2 \%$ bias limit in the concentration), the vitamin $\mathrm{E}$ solution used to generate aerosols was diluted for measuring the concentration using the spectrophotometer (about $2 \%$ bias limit in the concentration). With bias values mentioned in section 4.1.2.1 ( $Q_{s}$ is the volume flow rate of the jet $=5.44 \times 10^{-9} \mathrm{~m}^{3} / \mathrm{s}$ for the syringe pump setting of $9.9 \times 10^{-4} \mathrm{~cm}^{-4} / \mathrm{s}$ with $1 \%$ bias limit; $f$ is the vibration frequency of the frequency generator with $1 \%$ bias limit; $C$ is the volumetric concentration of the vitamin $\mathrm{E}$ in alcohol $C=0.000196 \times$ Absorbance at $285 \mathrm{~nm}$ with $2 \%$ bias limit), the total bias limit for the calculated aerodynamic diameter can be obtained using equation 4.11 :

$$
\begin{aligned}
& \frac{\beta_{d_{a}}}{d_{a}}=\frac{1}{3} \sqrt{\left(\frac{\beta_{Q_{S}}}{Q_{S}}\right)^{2}+\left(\frac{\beta_{f}}{f}\right)^{2}+\left(\frac{\beta_{C+I}}{C+I}\right)^{2}+\left(\frac{\beta_{\rho}}{\rho}\right)^{2}+\left(\frac{\beta_{C}}{C}\right)^{2}+\left(\frac{\beta_{C}}{C}\right)^{2}} \\
& =\frac{1}{3} \sqrt{(1 \%)^{2}+(1 \%)^{2}+(2 \%)^{2}+\left(\frac{1 \%}{0.92}\right)^{2}+(2 \%)^{2}+(2 \%)^{2}}=2 \%
\end{aligned}
$$


From the definition of inertial parameter, the bias limit in it is then:

$$
\frac{\beta_{I P}}{I P}=\sqrt{\left(\frac{2 \beta_{d_{a}}}{d_{a}}\right)^{2}+\left(\frac{\beta_{Q}}{Q}\right)^{2}}
$$

Combining all parameters discussed above together, the bias limit in the inertial parameter (I.P.) can be calculated:

$$
\frac{\beta_{I P}}{I P}=\sqrt{\left(\frac{2 \beta_{d_{a}}}{d_{a}}\right)^{2}+\left(\frac{\beta_{Q}}{Q}\right)^{2}}=\sqrt{(2 \times 2 \%)^{2}+(0.72 \%)^{2}}=2.92 \%
$$

Only the measured sizes are used for the presentation of the final data. It is conjectured that there was much variability in the dilution process, and possibly a higher uncertainty in the calibration constant of concentration versus absorbance than estimated. Thus it should be assumed that there is some uncertainty in the inertial parameter not captured by this uncertainty analysis.

\subsubsection{Total particle deposition fraction}

The total particle deposition fraction, $D F$, can be calculated by:

$$
D F=\frac{\sum_{i=1}^{3} \operatorname{Vol}_{n_{i}} A b_{n_{i}}}{\sum_{i=1}^{3} \operatorname{Vol}_{n_{i}} A b_{n_{i}}+\operatorname{Vol}_{f} A b_{f}}=\frac{\sum_{i=1}^{3} N_{n_{i}}}{\sum_{i=1}^{3} N_{n_{i}}+N_{f}}=\frac{N_{n}}{N_{n}+N_{f}}
$$

where, $\mathrm{Vol}_{n}, \mathrm{Vol}_{f}$ and $A b_{n}, A b_{f}$ are the volume and absorption of solutions obtained from the washing of nasal model and down-stream filter, respectively. The bias limit of $D F$ is: 


$$
\begin{gathered}
\beta_{D F}=\sqrt{\sum_{i=1}^{3}\left(\beta_{N_{n_{i}}} \frac{\partial D F}{\partial N_{n_{i}}}\right)^{2}+\left(\beta_{N_{f}} \frac{\partial D F}{\partial N_{f}}\right)} \\
\frac{\partial D F .}{\partial N_{n}}=\frac{N_{f}}{\left(N_{n}+N_{f}\right)^{2}}=\frac{\frac{N_{f}}{N_{n}} N_{n}}{\left(N_{n}+N_{f}\right)^{2}}=D F(1-D F) \frac{1}{N_{n}} \\
\frac{\partial D F}{\partial N_{f}}=\frac{-N_{n}}{\left(N_{n}+N_{f}\right)^{2}}=D F(1-D F) \frac{-1}{N_{f}} \\
\frac{\beta_{D F}}{D F}=(1-D F) \sqrt{\left(\frac{\beta_{N_{n}}}{N_{n}}\right)^{2}+\left(\frac{\beta_{N_{f}}}{N_{f}}\right)^{2}} \\
D F \\
=(1-D F) \sqrt{\sum_{i=1}^{3}\left(\frac{\beta_{v_{o m_{i}}}}{V o l_{n_{i}}}\right)^{2}+\left(\frac{\left.\beta_{v_{o f f}}\right)^{2}+\sum_{i=1}^{3}\left(\frac{\beta_{A b n_{i}}}{A b_{n_{i}}}\right)^{2}+\left(\frac{\beta_{A b f}}{A B_{f}}\right)^{2}}{V_{f}}\right.}
\end{gathered}
$$

Some estimates have been made to calculate the bias of total particle deposition fraction:

$$
\begin{gathered}
\frac{\beta_{V_{\text {Von }}}}{V o l_{n_{i}}}=1.4 \% \\
\frac{\beta_{V_{o l f}}}{V o l_{n_{f}}}=4.3 \% \\
\frac{\beta_{A b b_{i}}}{V o l_{A b_{i i}}}=\frac{\beta_{A b j}}{V o l_{A b_{f}}}=2 \%
\end{gathered}
$$

Then, the bias limit of the total particle deposition fraction can be calculated by:

$$
\begin{aligned}
& \frac{\beta_{D F}}{D F}=(1-D F) \sqrt{\sum_{i=1}^{3}(1.4 \%)^{2}+(4.3 \%)^{2}+\sum_{i=1}^{3}(2 \%)^{2}+(2 \%)^{2}} \\
& =6.35 \%(1-D F)
\end{aligned}
$$


In summary, the bias limits of the key parameters in the present experiment are summarized in Table 4.1.

Table 4.1 Bias limits of the key parameters of total particle fraction measurement

\begin{tabular}{|c|c|}
\hline Parameter & Bias limit \\
\hline$d_{a}$ & $2.0 \%$ \\
\hline$Q$ & $0.72 \%$ \\
\hline$I . P$. & $2.92 \%$ \\
\hline$D F$ & $6.35 \%(1-D F)$ \\
\hline
\end{tabular}

\subsubsection{Precision}

The precision error is random error and is different for every reading, and hence cannot be removed. It causes scatter in the total particle deposition fraction results of different experiments for the same experimental parameters (particle size and inhalation flow rate). In present experimental research, total particle deposition $(D F)$ for three inhalation flow rates $(90,60,30 \mathrm{~L} / \mathrm{min})$ was studied. For each flow rate, $5 \sim 7$ data points (supposedly with the same particle diameter and impaction parameter) were used. Three measurements were performed for each data point. When different experiments at the same data point have different particle diameters or impaction parameters, the average particle diameters and impaction parameters are used. The error caused by this is accounted for in the precision error. The precision limit of the measured results can be calculated using following equations:

$$
P_{\bar{r}}=\frac{t S_{\bar{r}}}{\sqrt{M}}
$$




$$
\begin{gathered}
S_{\bar{r}}=\left[\sum_{k=1}^{M} \frac{\left(r_{k}-\bar{r}\right)^{2}}{M-1}\right]^{1 / 2} \\
\bar{r}=\frac{1}{M} \sum_{k=1}^{M} r_{k}
\end{gathered}
$$

where, $\mathrm{t}$ is the coverage factor, $S_{\breve{r}}$ is the standard deviation for $\mathrm{M}$ readings of the results, and $\bar{r}$ is the averaged result.

For example, for the data point with inhalation flow rate of $60 \mathrm{~L} / \mathrm{min}$, particle diameter of $4.69 \mu \mathrm{m}$ and an impaction parameter of 1214.34 , the three readings gave an averaged total particle deposition fraction of 0.55 with a standard deviation of 0.0166 . The $\mathrm{t}$-value for 2 freedom degree and a $95 \%$ confidence interval was 4.32 , thus the uncertainty for this data point can be calculated: $P_{\bar{r}}=\frac{t S_{\bar{r}}}{\sqrt{M}}=\frac{4.32 \times 0.166}{\sqrt{3}}=0.041=4.1 \%$.

\subsubsection{Total uncertainty}

As discussed above, the bias error and precision error of the total particle deposition fraction can be determined. The total uncertainty of the total particle deposition fraction is the root sum of squares of the precision error and the bias error. Then the total uncertainty for each average result can be calculated using:

$$
U_{\bar{r}}^{2}=B_{r}^{2}+P_{\bar{r}}^{2}
$$

For example, using the same data point discussed in the previous section with inhalation flow rate of $60 \mathrm{~L} / \mathrm{min}$, particle diameter of 4.69 micron, impaction parameter of 1214.34 , the bias error is 0.009 , and the precision is 0.041 , there is a total uncertainty 
of $\sqrt{0.009^{2}+0.041^{2}}=0.044$. The detailed precision error, bias error and total uncertainty of experimental results as well as further discussion of the experimental results will be given in the following chapter. 


\section{Chapter 5: $\quad$ Experimental Deposition Results}

\subsection{Measured and simulated total particle deposition fraction}

The total deposition of particles $(D F)$ in the nasal cavity is a function of particle size and respiratory patterns. As mentioned in section 2.2 , it is common to define an impaction parameter $d_{a}^{2} Q=\rho_{p} d_{p}^{2} Q$ to analyse particle deposition fraction, where $d_{a}$ is the aerodynamic diameter of particles (usually in $\mu \mathrm{m}$ ), $\rho_{p}$ is the particle density in $\mathrm{g} / \mathrm{cm}^{3}$, $d_{p}$ is the particle diameter in $\mu \mathrm{m}$, and $Q$ is the inhalation flow rate in $L / \mathrm{min}$.

\subsubsection{Measured total particle deposition fraction}

The experimental results of total particle deposition fraction in the standardized nasal cavity, along with the calculated experimental uncertainties, are summarized in Table 5.1. Total absolute uncertainties in measured deposition fraction ranged from 0.011 to 0.063 ( 4.2 to $29.5 \%$ of the measured deposition fraction). It can be seen that for each flow rate, the total particle deposition fraction increase as the inertial impaction parameter increase. The trajectories of particles with large inertia are greatly determined by inertial forces and tend to deviate from flow streamlines. Thus, particles with large inertia deviate from flow streamlines, impact into airway surfaces and then deposit there.

In following sections, the current experimental data of the standardized human nasal cavity are compared with compared with in vivo and in vitro data of other researchers. 
As shown in , the current data are plotted in for flow rates of 30,60 , and $90 \mathrm{~L} / \mathrm{min}$, with two-dimensional error bars representing calculated total experimental uncertainties in both deposition fraction and impaction parameter.

Table 5.1 Experimental Results and Uncertainty

\begin{tabular}{|c|c|c|c|c|c|c|}
\hline $\begin{array}{c}\text { Flow Rate } \\
(\mathbf{L} \text { min) }\end{array}$ & $\begin{array}{c}\text { Particle } \\
\text { Size } \\
(\boldsymbol{\mu})\end{array}$ & $\begin{array}{c}\text { Impaction } \\
\text { Parameter } \\
\left(\mu \mathbf{m}^{2} \text { L/min) }\right.\end{array}$ & $\begin{array}{c}\text { Total } \\
\text { Deposition } \\
\text { Fraction }\end{array}$ & $\begin{array}{c}\text { Bias } \\
\text { Limit }\end{array}$ & $\begin{array}{c}\text { Precision } \\
\text { Uncertainty }\end{array}$ & $\begin{array}{c}\text { Combined } \\
\text { Uncertainty }\end{array}$ \\
\hline 90 & 5.00 & 2070.1 & 0.857 & 0.007 & 0.036 & 0.036 \\
\hline & 3.61 & 1080.1 & 0.533 & 0.016 & 0.061 & 0.063 \\
\hline & 3.06 & 772.8 & 0.423 & 0.016 & 0.041 & 0.044 \\
\hline & 2.49 & 511.5 & 0.237 & 0.012 & 0.039 & 0.040 \\
\hline & 1.74 & 250.8 & 0.116 & 0.007 & 0.028 & 0.029 \\
\hline & 6.77 & 2527.6 & 0.830 & 0.008 & 0.039 & 0.040 \\
\hline & 5.75 & 1823.0 & 0.690 & 0.013 & 0.029 & 0.032 \\
\hline & 4.69 & 1214.3 & 0.521 & 0.016 & 0.032 & 0.036 \\
\hline & 3.14 & 542.6 & 0.215 & 0.011 & 0.047 & 0.048 \\
\hline & 2.57 & 363.2 & 0.178 & 0.010 & 0.017 & 0.020 \\
\hline & 1.71 & 162.1 & 0.083 & 0.005 & 0.013 & 0.014 \\
\hline & 9.14 & 2305.7 & 0.790 & 0.011 & 0.047 & 0.048 \\
\hline & 8.20 & 1855.8 & 0.644 & 0.015 & 0.044 & 0.046 \\
\hline & 6.34 & 1110.1 & 0.473 & 0.016 & 0.042 & 0.045 \\
\hline & 5.23 & 756.2 & 0.332 & 0.014 & 0.046 & 0.048 \\
\hline & 3.09 & 263.5 & 0.102 & 0.006 & 0.032 & 0.032 \\
\hline & 2.11 & 123.3 & 0.061 & 0.004 & 0.010 & 0.011 \\
\hline
\end{tabular}

\subsubsection{Comparison with in-vivo data}

In Figure 5.1, data are compared with in vivo measurements of Landahl and Black (1947), Pattle (1961), Hounam et al. (1969), Giacomelli-Maltoni et al. (1972), and Heyder and Rudolf (1977). The solid line from Cheng (2003) representing the empirical fit to cited in vivo data is also showed for comparison purpose. 
It is worth noting from Figure 5.1 that the in vivo data scatter in a significant range, especially considering that data are plotted on semi-log axes. Results for present in vitro measurements using the Carleton-Civic standardized model effectively capture the sigmoid trend but fall on the lower bound of the scattered in vivo data.

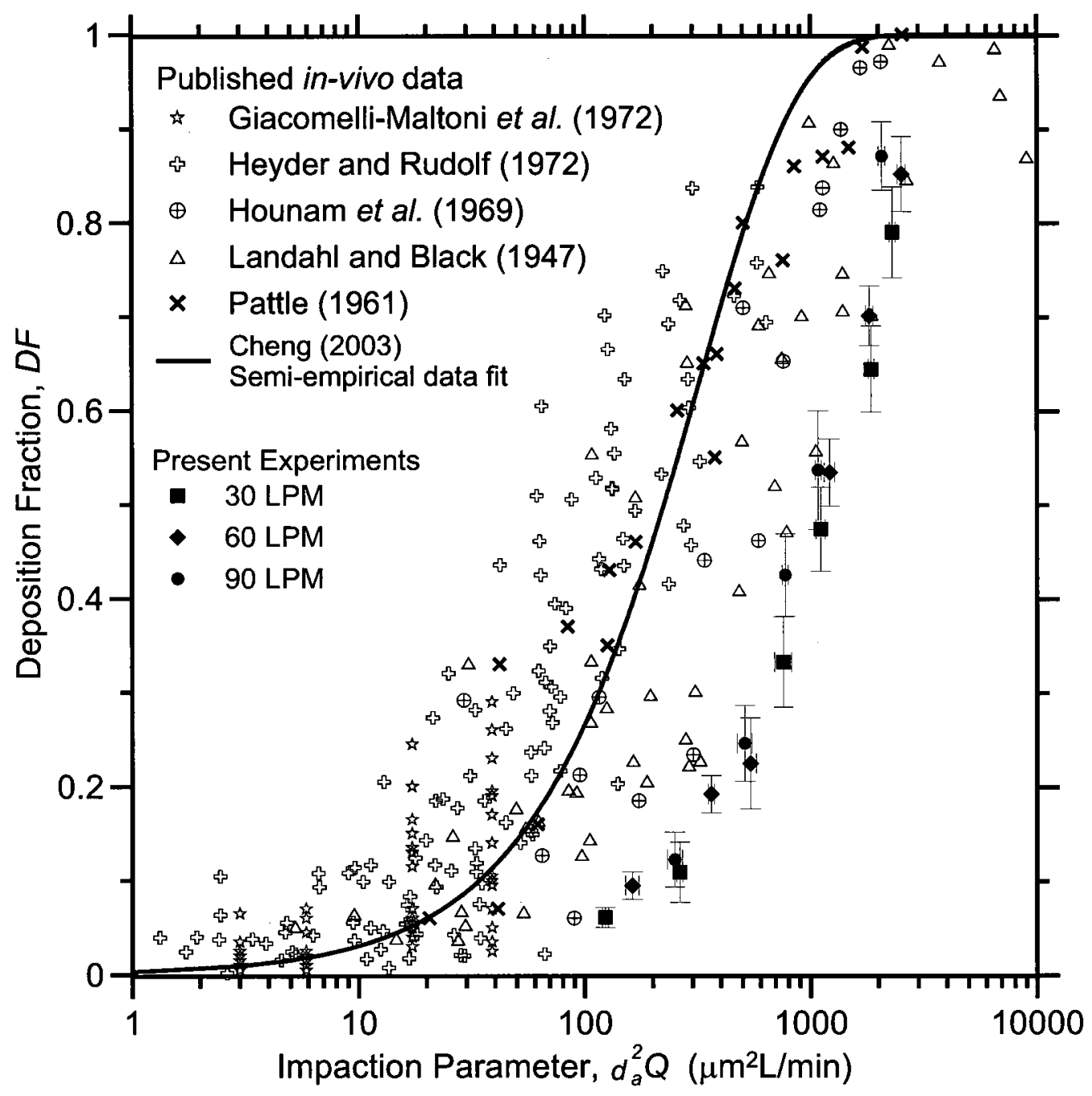

Figure 5.1 Experimental results of total particle deposition fraction compared with published in vivo data

This difference is not unexpected because of the different principles of in vivo and in vitro measurements. In vivo data are typically derived from concentration measurements 
before inhalation into the nose and after exhalation through the mouth (Landahl and Black, 1947; Pattle, 1961; Hounam et al., 1969; Giacomelli-Maltoni et al., 1972; Heyder and Rudolf, 1977), and as such calculated depositions fractions from in vivo measurements would include any additional deposition in the nasopharynx and in the oral cavity. Moreover, in in vivo experiments, the presence of hairs in the nostrils would increase particle filtration, particularly for larger particles (see Landahl and Tracewell, 1949; Scott et al., 1978; Swift and Kesavanathan, 1996). Finally, differences in nasal cavity "wall roughness" between the hard surfaced in vitro replicas and actual human subjects might also affect deposition

\subsubsection{Comparison with published in vitro data}

As shown in Figure 5.2, measured data in the standardized nasal cavity are compared with published in vitro results from Swift (1991), Lennon et al. (1998), Zwartz and Guilmette (2001), Cheng et al. (2001), Kelly et al. (2004), and Garcia et al. (2009). The semi-empirical fit line to cited in-vivo data, as summarized by Cheng (2003), is also shown for comparison. With the exception of the data from Swift (1991), which were obtained using a hand-carved replica, all of the in vitro data follow a similar trend, which itself falls below the summary in vivo curve. Thus, the difference between in vivo and in vitro derived data is consistent in the literature, and is attributable to the combination of effects cited in Section 5.1.1.1.

Observing in vitro data only, the current measured deposition results for the standardized geometry cross through the bulk of the available data, while showing comparatively less deposition at high values of the inertial impaction parameter (i.e. $d_{a}^{2} Q$ 
$>1000$ ). Given the range of different flow rates and particle sizes among these in vitro data sets, the relative lack of scatter in deposition fraction when plotted against the inertial parameter, confirms that inertial impaction is the dominant mechanism of particle deposition.

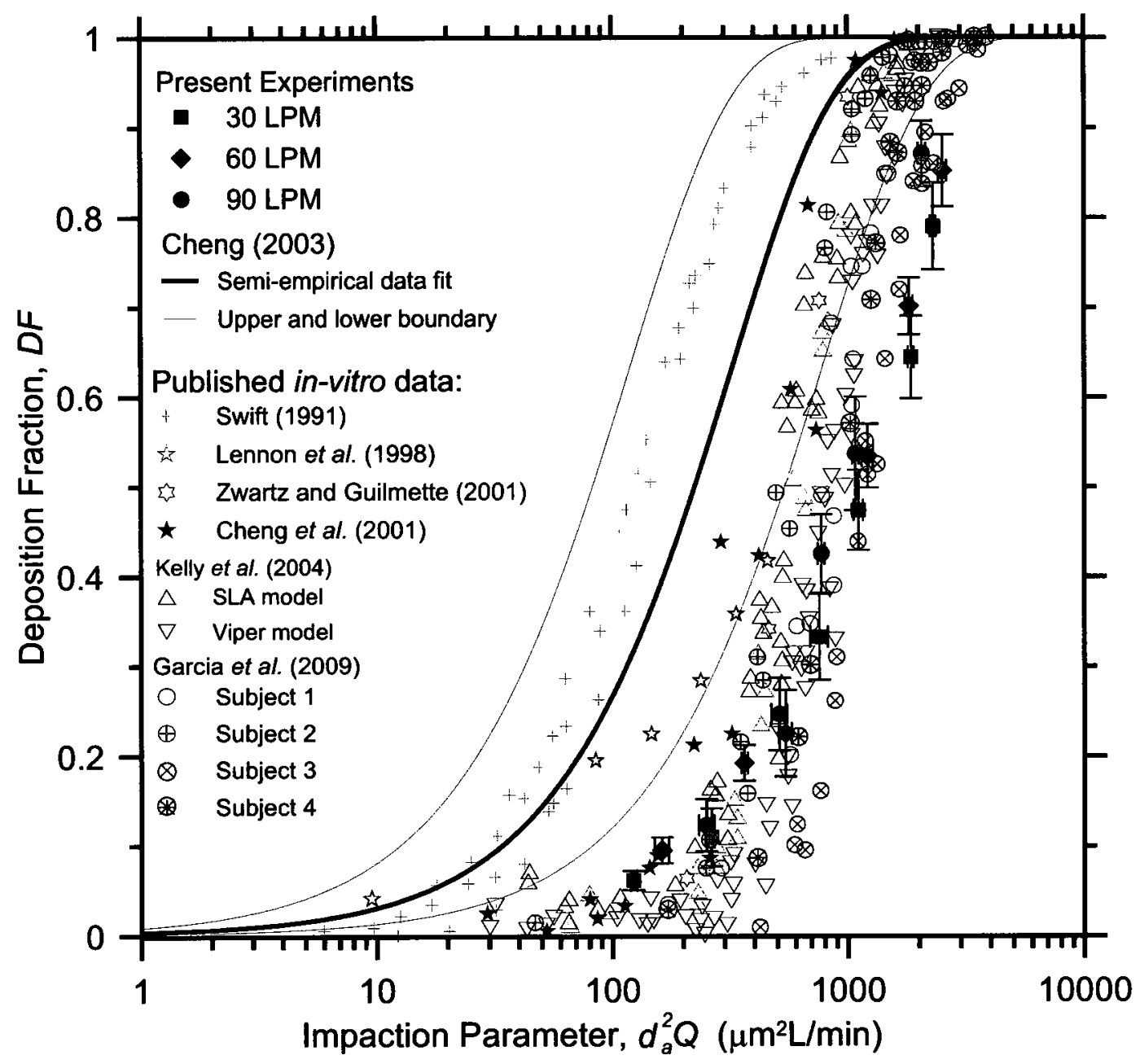

Figure 5.2 Experimental results of total particle deposition fraction compared with published in vitro data

While the agreement among different data sets for different geometries is reasonable given the complexity of the measurements, there are several potential reasons why these results may vary. First is the issue of experimental uncertainty. For the current work, 
total experimental uncertainties are indicated with vertical error bars. Although detailed uncertainty estimates have not generally been reported in the literature, by considering repeated measurements by different researchers using the same geometry, it is possible to qualitatively estimate their significance. In Figure 5.2, all of the data points shown in blue were obtained by a variety of researchers using geometries derived from the same set of CT scans for a single human subject originally reported by Swift (1991). These include the SLA and Viper models of Kelly et al. (2004), the first model of Garcia et al. (2009), and the models of Zwartz and Guilmette (2001), Cheng et al. (2001), and Swift (1991). As discussed in Section 2.3.2.2, differences in measured deposition fraction among these studies can be attributed to a combination of experimental uncertainties and variations in wall roughness due to different manufacturing methods (Kelly et al., 2004). The span of these previous in vitro data is not insignificant, suggesting that experimental uncertainties (including wall roughness effects) must be acknowledged when comparing different data sources.

Variation in deposition patterns could also be expected among in-vitro models derived from different geometries. The latter three models of Garcia et al. (2009) and the model of Lennon et al. (1998) originate from different subjects and thus also reflect intersubject variability. Focusing just on data from subjects 2-4 of Garcia et al. (2009), for which any potential systematic errors should be consistent, the variability among these subjects spans the main data band in Figure 5.2. Considering also the data from Lennon et al. (1998), it can be seen that inter-subject variability is likely the dominant source of scatter, which highlights the role and significance of our novel standardized geometries in research. Ideally, one would hope that the in vitro deposition data for the standardized 
model would fall on the center of data derived from these different individuals. However, since the number of different subjects represented in the available cited data is still quite small (i.e. 5 individuals), and effects of inter-subject variability in these data are quite significant, the level of agreement is not unreasonable.

Finally, besides issues of uncertainty, wall-roughness, and inter-subject variability, there is still the possibility that the variance in deposition fraction at high values of the impaction parameter is in fact statistically significant, and that some aspect of the standardized geometry itself results in difference. Nevertheless, it is reassuring that the data from the standardized model crosses through the bulk of the published in vitro data while showing a similar sigmoid trend as noted above.

\subsubsection{Measured regional particle deposition fraction}

In addition to total particle deposition fraction, regional distribution of deposited particles in the human nasal cavity is very important when studying nasal drug delivery. As detailed in the discussion of the experimental apparatus, the physical replica was split into three parts so that regional deposition data could be collected and analyzed. The regions were designed based on the physiology of nasal cavity, and were not of equal size. The anterior region included the parts of the nose and passageways up to the nasal valve; the middle region included the turbinates up to the choana, and the posterior region included the small region in the vicinity of the nasopharynx up to the end of the model.

Figure 5.3a-c shows the measured regional particle deposition fraction for inhalation flow rates of 30,60 , and $90 \mathrm{~L} / \mathrm{min}$. Despite the different flow rates, the regional particle deposition fraction has the same trends for anterior, middle and posterior sections of the 
cavity. Particle deposition fraction in the anterior part of nasal cavity increases with increasing particle size, and is the major part of the total deposition fraction. For the middle region, the peak deposition occurs when the impaction parameter is equal to approximately $1500 \mathrm{~mm}^{2} \mathrm{~L} / \mathrm{min}$. For the posterior section, the deposition fraction does not vary significantly with impaction parameter and is sufficiently small that it can be neglected. Further discussion of the measured regional deposition fraction (including comparison with simulated results) is shown in Chapter 7.
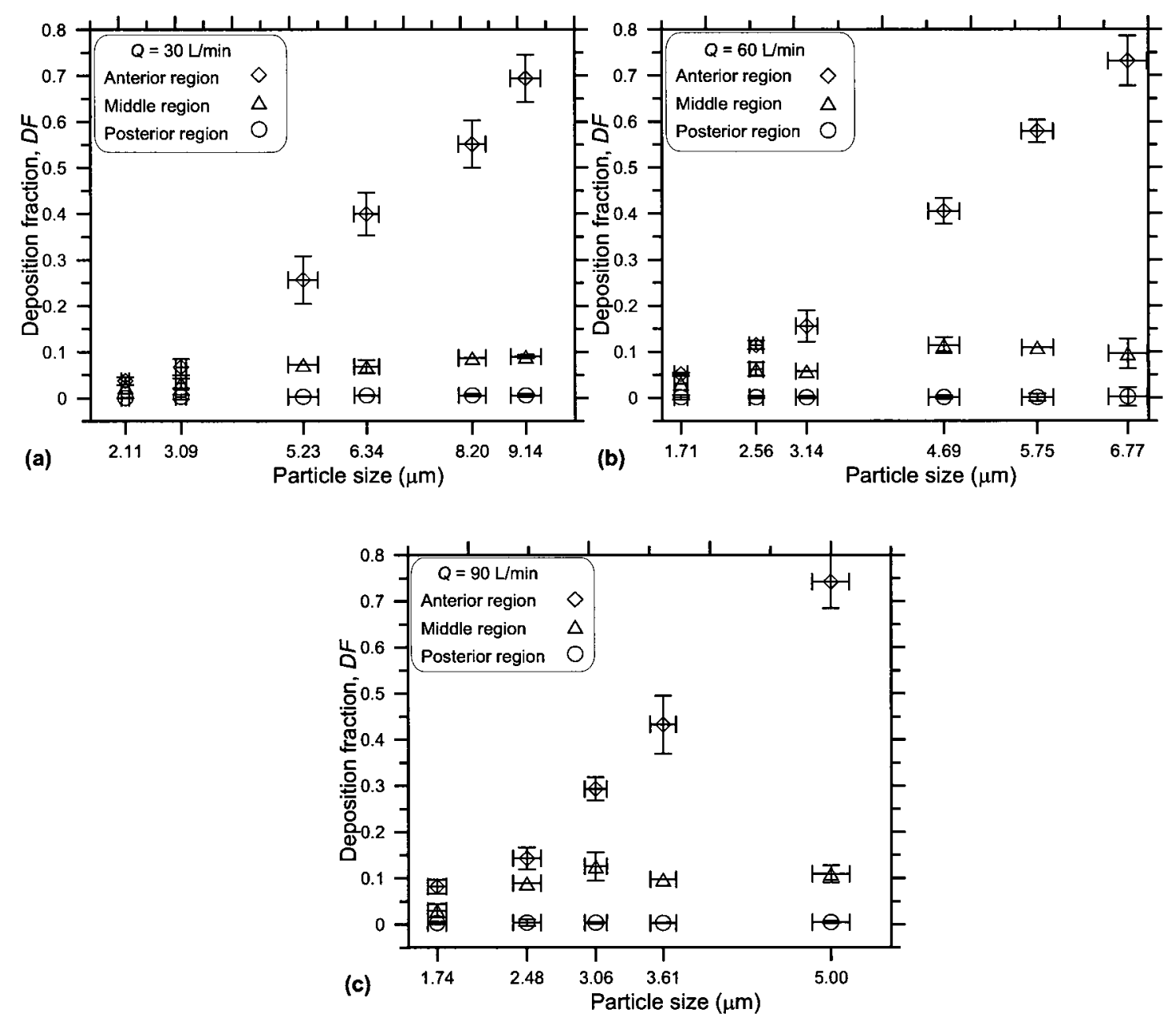

Figure 5.3 Experimental measurements of regional particle deposition fraction in the Carleton-Civic standardized nasal cavity geometry 


\section{Chapter 6: $\quad$ Numerical Simulation Methodology}

\subsection{Theory}

\subsection{The motion of particles}

To estimate the net force on an immersed particle, we consider all individual component forces, which may arise from pressure gradients and relative motion of the fluid and particle, and may be functions of position, velocity, and acceleration. These forces are summarized in Table 6.1, where $V$ is the volume of the body, $\rho$ is the density of the fluid, $A_{D}=C_{D} A$ is the drag area, $C_{D}$ is the steady drag coefficient, $\mathrm{A}$ is the projected area of the object, $\left(a_{F}\right)_{G}$ is the acceleration of fluid relative to Earth and $\left(a_{F}\right)_{B}$ is the acceleration of fluid relative to the body. It is also possible that the rate of change of acceleration could influence the net force, or even higher motion derivatives might be significant.

\section{Table 6.1 Force exerted on immersed objects}

\begin{tabular}{|l|c|c|c|}
\hline Pressure Gradient & $1 . B_{1}=\rho V g$ & $2 . B_{2}=-V(d p / d x)$ & 3. $A_{2}=\rho V\left(a_{F}\right)_{0 G}$ \\
\hline Relative Motion & & $4 . D=0.5 \rho\left(u_{F}\right)^{2} A_{D}$, etc. & 5. $A_{1}=m_{a}\left(a_{F}\right)_{B}$ \\
\hline
\end{tabular}

Force type 1 exists in the absence of particle and fluid motion, which is the familiar buoyancy force $B=\rho V g$ arising from the pressure gradient $\rho g$ caused in the fluid by gravity. Force type 2 and 3 also arise from pressure gradients in the fluid, but type 2 arises from friction, e.g. on the walls of a wind tunnel, but not on the object itself, and type 3 arises from the pressure gradient in the bulk fluid. These two forces are also called buoyancy forces and all the type 1,2 and 3 results in the concept of generalized buoyancy. Type 4 represents the forces arising directly from the velocity of the fluid 
relative to the selected free body, $\left(\breve{u_{F}}\right)_{B}$. These include forces like lift and drag (roughly dependent on $u^{2}$ ), or the viscous damping force used in vibration analysis (proportional to $u$ ). Additionally, turbulent dispersion force also belongs to this type of force. These are only important for small objects (such as water drops smaller than $100 \mu m$ in air). Turbulent dispersion force brings additional dispersion of particles from regions with high volume fraction to regions with low volume fraction because of turbulent fluctuations. The type 5 force is composed of forces that arise from changes of $\left(\overrightarrow{u_{F}}\right)_{B}$, i.e., acceleration of the fluid relative to the free body. This type of force can arise from an absolute acceleration of the object or of the fluid, or sometimes both at once.

When an object moves within a fluid, the fluid moves around the object, and then the fluid itself must have kinetic energy. If the speed distribution in the fluid is known, then we can calculate the velocity field of the fluid and obtain the kinetic energy of the fluid. For the idealized case of zero viscosity, an object has no drag, the velocity field and the kinetic energy of the fluid can be determined. The fluid kinetic energy of a moving object, for which the surrounding fluid speed is directly proportional to the object speed, is directly proportional to the object kinetic energy, and can be defined by following equation:

$$
K=\frac{1}{2} m u^{2}+\frac{1}{2} m_{a} u^{2}
$$

where, the empirical constant $m_{a}$ is the added mass, or the added inertia.

Provided that the acceleration of the fluid relative to the free body is used, we obtain:

$$
A_{1}=m_{a}\left(a_{F}\right)_{B}
$$


In practice, this is often quoted as the added-mass coefficient,

$$
C_{a}=\frac{m_{a}}{\rho V}
$$

where, $\rho$ is the fluid density and $V$ is the displacement volume of the object, i.e., $\rho V$ is the true mass of fluid displaced by the object. The added mass coefficient $C_{a}$ for a sphere is 0.5 .

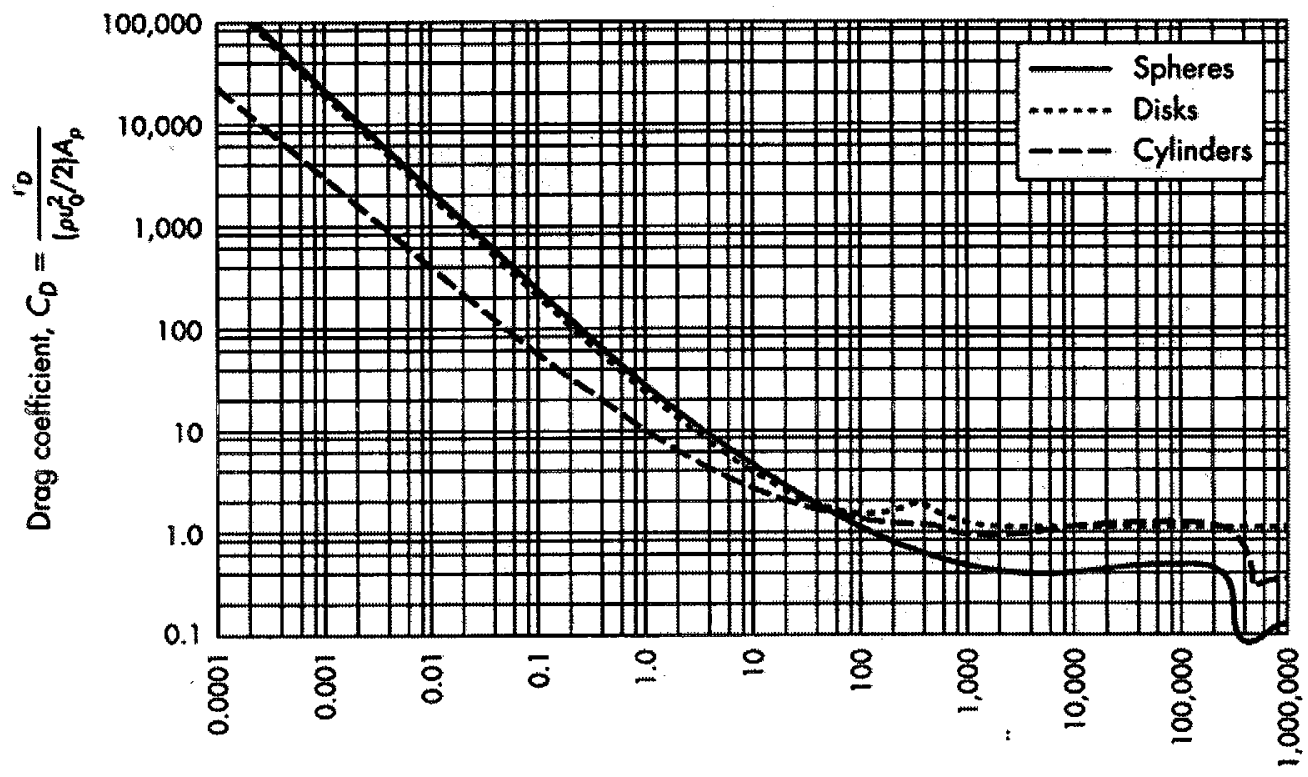

Reynolds number, $R e_{\rho}=\frac{D_{p} \rho v_{0}}{\mu}$

Figure 6.1 Drag coefficient (Lapple and Shepard, 1940)

Drag force is a force parallel to the fluid velocity, which the fluid exerts over the body and is given by,

$$
D=\frac{1}{2} C_{D} \rho\left(u_{F}\right)_{B}^{2} A
$$


where, $C_{D}$ is the drag coefficient. Figure 6.1 6.1 shows the drag coefficient of spheres, disks and cylinders as a function of the particle's Reynolds number (Lapple and Shepard, 1940).

For a particle with a Reynolds number less than 1 , Stokes found that the drag force is,

$$
D=6 \pi a \mu U
$$

where, $a$ is the radius of the sphere, $U$ is the relative velocity between the sphere and the fluid. For sparsely distributed spherical particles, several empirical correlations are available, and Schiller and Naumann (1933) proposed,

$$
C_{D}=\frac{24}{\operatorname{Re}}\left(1+0.15 \mathrm{Re}^{0.687}\right) \text { for } 0<\operatorname{Re} \leq 800
$$

which is available in ANSYS CFX (Canonsburg, PA) and is adopted in the present study.

\subsubsection{Particle equation of motion}

Based on Newton's second law, the acceleration of a particle is proportional to the resultant of all the forces acting on the particle:

$$
\sum \vec{F}=m \vec{a}
$$

Basset (1888) and Ossen (1927) applied Newton's second law of motion and derived an equation for a particle moving under the influence of gravity in a turbulent fluid at rest. Tchen (1947) extended their work and proposed an equation of motion for a small rigid spherical particle in a moving fluid: 


$$
\begin{aligned}
& \frac{\pi}{6} d_{p}^{3} \rho_{p} \frac{d u_{p}}{d t}=3 \pi \mu d_{p}\left(u_{f}-u_{p}\right)-\frac{\pi}{6} d_{p}^{3} \rho_{f} \frac{d u_{f}}{d t}+\frac{1}{2} \frac{\pi}{6} d_{p}^{3} \rho_{f}\left(\frac{d u_{f}}{d t}-\frac{d u_{p}}{d t}\right) \\
& +\frac{2}{3} d_{p}^{2} \sqrt{\pi \mu \rho_{f}} \int_{t_{0}}^{t} \frac{d u_{f} / d t^{\prime}-d u_{p} / d t^{\prime}}{\sqrt{t-t^{\prime}}}+\frac{\pi}{6} d_{p}^{3}\left(\rho_{f}-\rho_{p}\right) g
\end{aligned}
$$

where

$$
\begin{aligned}
& d_{p}=\text { the diameter of the particle } \\
& u_{p}=\text { the velocity of the particle } \\
& u_{f}=\text { the velocity of the fluid phase } \\
& \rho_{p}=\text { the density of the particle } \\
& \rho_{f}=\text { the density of the fluid phase } \\
& t_{0}=\text { the starting time } \\
& \mu=\text { the viscosity of fluid phase }
\end{aligned}
$$

In Equation 6.8, the term on the left-hand side of the equal sign is the force required to accelerate the particle. The first term on the right-hand side of the equal sign is the linear viscous resistance force according to Stoke's law. The second term represents the resultant of the pressure gradient in the fluid surrounding the particles, which is a direct effect of the fluid acceleration. The third term represents the surplus of inertia caused by the pressures resulting from the relative acceleration. This term is equivalent to the inertia of the virtual, "added" mass attached to the particle. The fourth term is usually called the "Basset" term and takes into account the effect of the deviation in flow pattern from steady state. It gives the force due to the history of the particle; thus, it is also 
called the history term. The last term is due to the external potential force (usually the gravitational force).

There are several assumptions made for the derivation of Equation 6.8:

(1) The turbulence of fluid field is homogeneous, steady;

(2) The domain of turbulence is infinite;

(3) The particle is spherical and follows Stokes's Law;

(4) There is no mutual action between particles; and

(5) The diameter of particle must be many times smaller than the Lagrangian eddy length scale as given by equation (6.17).

Several authors extended Tchen's work and proposed other equations. Corrsin and Lumley (1956) modified Tchen's equation by including a term for turbulence pressure gradients. Maxey and Riley (1983) developed equations of motion for a small rigid particle with relative motion of low Reynolds number in a non-uniform flow.

Equation 6.9 is the equation of motion of particle for finite Reynolds number proposed by Mei (1996):

$$
\begin{aligned}
& \frac{4}{3} \pi a^{3} \rho_{p} \frac{d V}{d t}=\frac{4}{3} \pi a^{3}\left(\rho_{f}-\rho_{p}\right) g+6 \pi \mu a \phi(\operatorname{Re})(U-V) \\
& +6 \pi \mu a \int_{t_{0}}^{t} K(t-\tau) \frac{d(U-V)}{d \tau} d \tau+\frac{2}{3} \pi a^{3} \rho_{f}\left(\frac{D U}{D t}-\frac{d V}{d t}\right)+\frac{4}{3} \pi a^{3} \rho_{f} \frac{D U}{D t}
\end{aligned}
$$

where $V$ and $U$ are the instantaneous and local velocities of the particle and fluid, respectively; $a$ is the radius of particle and $g$ is the gravitational acceleration. In Equation 6.9, the first term on the right-hand side is the body force (gravity minus buoyancy force); the second term is the drag force; the third term is the Basset history 
force; the forth term is the added-mass force and the fifth term is the force due to the fluid pressure gradient and viscous stresses. External forces can be added to the above equation, such as the Saffman lift force due to the velocity gradient transversal to the particle's direction (Saffman, 1965) and the lift force due to the rotation of the particles (Magnus effect).

Considering particles in the range of 0.354 to $16.0 \mu \mathrm{m}$ with low density ratios $\left(\rho_{f} / \rho_{p}\right.$ $<<1$ - making several terms negligible in the particle equation of motion) and taking into account only viscous drag and gravity, the following three-dimensional Lagrangian equations describing the movement of a particle in a Cartesian coordinate system are adopted for the present simulation (Maxey and Riley, 1983; Kim et al., 1998; Mei, 1996; Matida et al., 2004 ):

$$
\begin{gathered}
\frac{d u_{p}}{d t}=\frac{\left(u_{f}-u_{p}\right)}{\tau_{p} / \alpha}, \\
\frac{d v_{p}}{d t}=\frac{\left(v_{f}-v_{p}\right)}{\tau_{p} / \alpha}, \\
\frac{d v_{p}}{d t}=\frac{\left(w_{f}-w_{p}\right)}{\tau_{p} / \alpha}-g\left(1-\frac{\rho_{f}}{\rho_{p}}\right),
\end{gathered}
$$

where the particle relaxation time (the time a particle with an initial velocity, $U_{p 0}$, would travel through a stagnant fluid under the conditions of Stokes drag) is

$$
\tau_{p}=\frac{\rho_{p} d_{p}^{2}}{18 \mu_{f}}
$$

and

$$
\alpha=1+0.15 \operatorname{Re}_{p}^{0.687}
$$

where $\operatorname{Re}_{p}$ is the particle's Reynolds number. 


\subsection{Simulation approach}

\subsubsection{RANS/EIM approach}

\subsubsection{Averaged Navier-Stokes Equations (RANS)}

The time averaged continuity equation and momentum equations are given by:

$$
\begin{gathered}
\frac{\partial \rho}{\partial t}+\rho \frac{\partial \bar{U}_{i}}{\partial x_{i}}=0 \\
\frac{\partial \bar{U}_{i}}{\partial t}+\bar{U}_{j} \frac{\partial \bar{U}_{i}}{\partial x_{j}}=-\frac{1}{\rho} \frac{\partial \bar{P}}{\partial x_{i}}+\frac{1}{\rho} \frac{\partial}{\partial x_{j}}\left(\mu \frac{\partial \bar{U}_{i}}{\partial x_{j}}+\mu \frac{\partial \bar{U}_{j}}{\partial x_{i}}-\rho \bar{u}_{i} u_{j}\right)
\end{gathered}
$$

where the new term, $\rho \overline{u_{i} u_{j}}$, is called the Reynolds stress tensor. The number of unknowns (ten: three velocity components, pressure, and six stresses) is larger than the number of equations (four: the continuity equation and three components of the NavierStokes equations), which leads to a closure problem. Therefore, the Reynolds stress term has to be obtained through modeling.

\subsubsection{1 k-w SST (Shear Stress Transport) Model}

As mentioned in Table 1.1, airflow in the human nasal cavity is highly complex and in the laminar to turbulent flow regimes. In order to capture the airflow structures in the human nasal cavity, the $k-\omega$ SST (Shear Stress Transport) model was selected here. SST is a blend between $k-\omega$ near the wall and $k-\varepsilon$ at the core of the domain. The standard $k-\omega$ model is used in the prediction of most turbulent flow calculations because of its robustness, economy, and reasonable accuracy for a wide range of flows. However, the 
model performs poorly when faced with non-equilibrium boundary layers. It tends to predict the onset of separation too late and to under-predict the amount of separation. Separation influences the overall performance of many devices, and also has a strong influence on other effects, such as wall heat transfer and multi-phase phenomena. This is undesirable and in order to solve the problem, a blending between $k-\omega$ near the wall and $k-\varepsilon$ at the core of the domain was developed. The $k-\omega$ based SST model accounts for the transport of the turbulent shear stress and gives highly accurate predictions of the onset and the amount of flow separation under adverse pressure gradients. The governing equations of the SST model are described in Appendix A.

\subsubsection{Eddy Interaction Model (EIM)}

The Eddy interaction model (EIM), as shown in Figure 6.2, is a Lagrangian method of simulation used to numerically track liquid or solid particles released in turbulent flows. In EIMs, (Zhou and Leschziner, 1991; Wang and Stock, 1992; Graham and James, 1996; Hennick and Lightstone, 2000), it is assumed that one particle is allowed to interact successively with various eddies, which means a particle is always within a single turbulent eddy. Each eddy has its characteristic lifetime, length, and velocity scale from the primary flow calculation results. The end of interaction between the particle and one eddy occurs when the lifetime of the eddy is over or when the particle crosses the eddy. At this instant, a new interaction with the particle and a new eddy is started. The trajectory of this particular particle is obtained by solving the particle equation of motion. In order to obtain velocity or deposition statistics, hundreds of particles must be released into the flow. The local fluctuating velocities are obtained by multiplying the root-mean 
square (RMS) fluid fluctuating velocity by random numbers $\left(N_{u}, N_{v}, N_{w}\right)$ generated from a Gaussian probability density function of zero mean and unity standard deviation at the start of one eddy-particle interaction. The random numbers are maintained constant during one eddy interaction, while respective RMS values are varied according to the particle position (see MacInnes and Bracco, 1992).

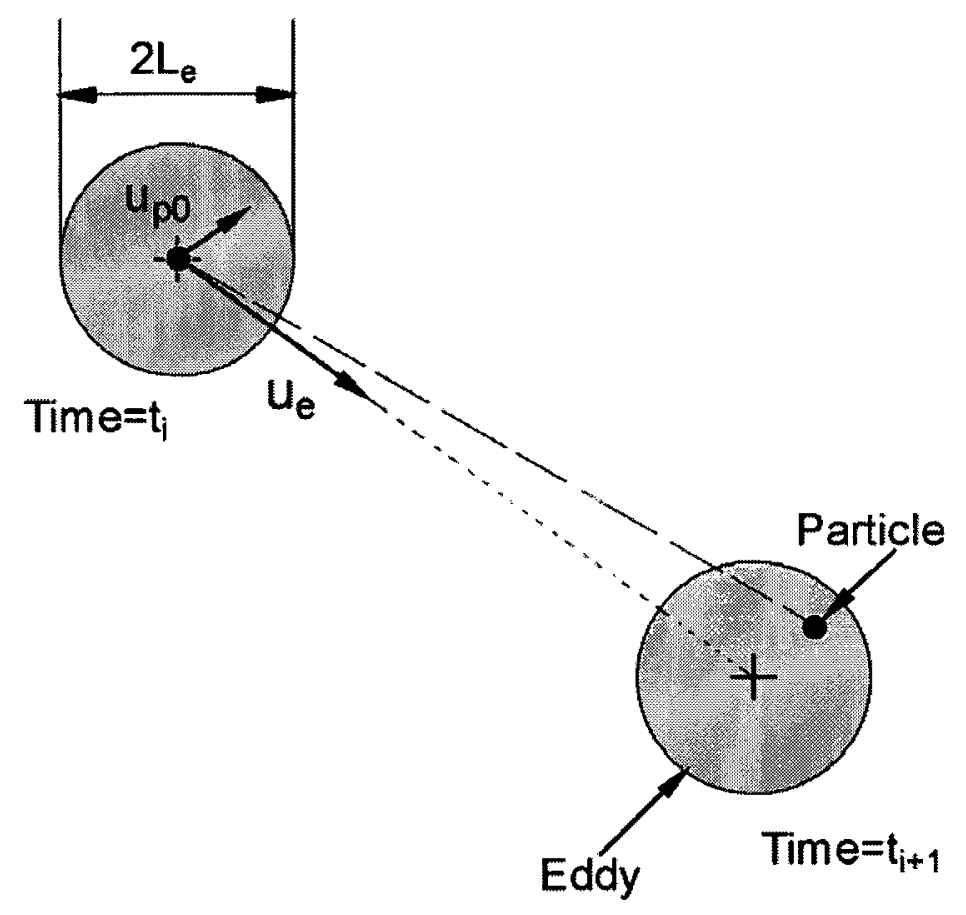

Figure 6.2 Eddy-particle interaction model

\subsection{Characteristic length, time and velocity scales of fluid eddies}

On the basis of the method of Gosman and Ionannides (1981), Schuen et al. (1983) simulated the particle dispersion in a turbulent round jet, where they used a $k-\varepsilon$ turbulence model along with the following characteristic length, time and velocity scales of fluid eddies: 


$$
\begin{aligned}
& L_{e}=C_{\mu}^{3 / 4} \frac{k^{3 / 2}}{\varepsilon} \\
& \tau_{e}=\frac{L_{e}}{(2 k / 3)^{1 / 2}} \\
& u_{e}=\left(\frac{2 k}{3}\right)^{1 / 2}
\end{aligned}
$$

where, $k$ is the local turbulent kinetic energy, $\varepsilon$ is the turbulent viscous dissipation, and $C_{\mu}$ is a turbulence constant, which was chosen to relate the characteristic length scale to the eddy dissipation length (Hinze, 1975). During one eddy-particle interaction, $\tau_{e}$ remains constant.

Although lifetime scales, $\tau_{e}$, can be randomized using an exponential probability density function, Graham and James (1996) pointed out that in the case when lifetime scales $\tau_{e}$ are specified to be constant in time, the choice of $\tau_{e}=2 \tau_{L}$ ensures dispersion consistency. Here $\tau_{L}$ is the Lagrangian fluid time scale. Graham and James (1996) also concluded that to reduce computational costs, the constant eddy lifetime scheme is preferred over an exponentially decaying lifetime scheme.

Considering that the Lagrangian and the Eulerian integral time scales are proportional in homogeneous isotropic stationary turbulence (HIST), Kallio and Reeks (1989) have estimated the Lagrangian integral time scales by,

$$
\tau_{L}=\beta \tau_{E} \cong 0.8 \beta \frac{k}{\varepsilon}
$$

Here, $\beta$ is a function of the turbulent Reynolds number $\left(\operatorname{Re}_{\lambda}=u^{\prime} \lambda / v\right)$, varying from 0.6 to 0.3 for $\operatorname{Re}_{\lambda}=20$ to 70 , according to Sato and Yamamoto's (1987) measurements. 
Hinze (1975) has proposed $\beta$ being approximately 0.4. According to Hinze (1975), the Lagrangian time scale is the largest interval in time that a Lagrangian particle will continue moving in that direction.

Since the particles used in the present calculation are relatively small, crossing trajectory effects are not relevant and the length scales can be neglected (Matida, 2000). The following characteristic scale of fluid eddies are used:

$$
\tau_{e}=2 \tau_{L}=0.2 k / \varepsilon
$$

for the time scale, with $\tau_{e}$ maintained constant during one eddy-particle interaction and

$$
u_{e}=\left(\frac{2 k}{3}\right)^{1 / 2}
$$

for the eddy characteristic velocity scale.

\subsection{Local fluctuation velocities of the eddy}

The fluid velocity components in Equations $6.12-6.14$ are instantaneous quantities composed of the mean part and the fluctuating part; for example:

$$
u_{f}=U_{f}+u_{f}^{\prime}
$$

The local fluctuation velocities of the eddy are generated at the start of each interaction and are given as follows:

$$
\left(u_{f}^{\prime}, v_{f}^{\prime}, w_{f}^{\prime}\right)=\left(N_{u} u_{r m s}^{\prime}, N_{v} v_{r m s}^{\prime}, N_{w} w_{r m s}^{\prime}\right)
$$


where, $u_{r m s}^{\prime}, v_{r m s}^{\prime}, w_{r m s}^{\prime}$ are the RMS values of the fluctuating velocities, $N_{u}, N_{v}$ and $N_{w}$ are the random numbers generated from a Gaussian probability density function of zero mean and unity standard deviation. In the present work, for the sake of simplicity, the isotropic decomposition of the turbulence is adopted:

$$
u_{r m s}^{\prime}=v_{r m s}^{\prime}=w_{r m s}^{\prime}=\left(\frac{2 k}{3}\right)^{\frac{1}{2}}
$$

Strictly speaking, the word random is reserved for the output of a random physical process, like the time between clicks of a Geiger counter placed next to a radioactive element. The term quasi-random is sometimes used for computer-generated sequences, which will be entirely predictable, hence not truly "random". There are many statistically well-tested "random number generators" available in the literature (see Press et al., 1989 and Knuth, 1981), such as uniform derivates, exponential deviates and normal (Gaussian) deviates, that can be used with some confidence.

\subsubsection{Large eddy simulation}

In additional to the RANS/EIM approach, the standard LES particle simulation is also performed on the same grid $(4,000,000$ elements) for comparison purposes. Large eddy simulation (LES) is a technique intermediate between the direct numerical simulation (DNS) of turbulent flows and the solution of the Reynolds-averaged equations (RANS).

In LES, the contribution of the large, energy-carrying structures to momentum and energy transfer is computed exactly, and only the effect of the smallest scales of turbulence is modeled. Since the small scales tend to be more homogeneous and 
universal, and less affected by the boundary conditions than the large ones, there is hope that their models can be simpler and require fewer adjustments when applied to different flows than similar models for the RANS equations.

LES is very similar to Direct Numerical Simulation (DNS) in that it provides a threedimensional, time dependent solution of the Navier-Stokes equations. Thus, fairly fine meshes are still required for LES. However, LES can be used at much higher Reynolds numbers than DNS. Ideally, in fact, if the small scales obey inertial-range dynamics, the cost of a computation is independent of $\operatorname{Re}$ (not, however, if a solid boundary is present). The standard subgrid scale model proposed by Smagorinsky (1963) and Lilly (1996) with a wall damping function is used here. The filtered equations are shown in Appendix B.

The simulation was initialized from a converged RANS velocity field as described in the previous section 6.3.1. In present work, "Frozen-domain" particle tracking method was used. Contrary with the dynamic LES (in which several groups of particles are released at different time steps, and are tracked over time), "Frozen-domain" is a threedimensional fluid domain whose values of the velocity are kept as the instantaneous values at the particular time step when the tracking begins. Thousands of particles were released and fully tracked in one iteration in a "frozen" LES computational domain and deposition statistics were gathered. This "frozen" calculation was made possible (in CFX 11.0) by tracking all particles in a single steady-mode iteration without any eddy interaction model. The reason for adopting the "Frozen-domain" particle tracking method here is that, when compared with the dynamic LES particle tracking approach, it is much more economical while yielding results very similar to those of the dynamic tracking approach (Breuer et al., 2006). 


\subsection{Simulation domain and method}

\subsubsection{Generation of mesh}

The Carleton-Civic standardized nasal model was meshed using ICEM-CFD (Ansys, Inc.). The surface and volume mesh were initially generated using tetrahedral elements and subsequently three layers of prismatic elements were created near the wall using $10 \%$ of the size of tetrahedral elements as initial height and 1.2 as the height ratio.

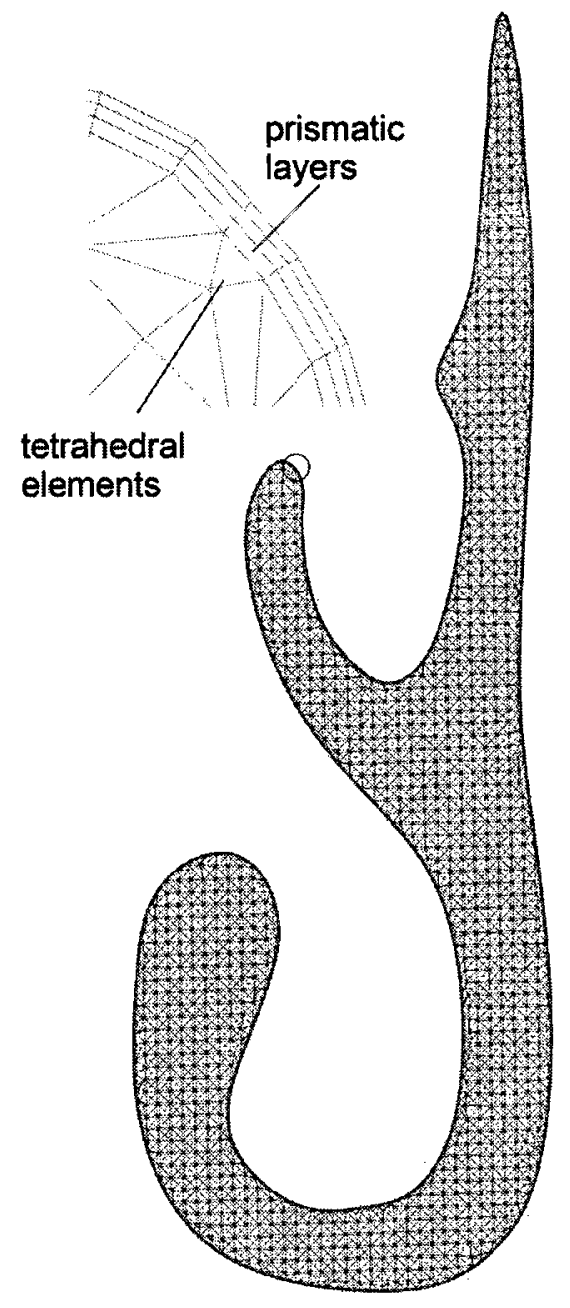

Figure 6.3 Sample of terahedual and prismatic elememts for 4000000 elememts 
The numerical accuracy can be enhanced with a highly refined mesh density of prismatic layers along the walls. Analysis of grid convergence was performed by considering pressure drop, mean velocity distribution at an arbitrary location and particle deposition for four different grid sizes (approximately 300 000, 1000000,2000000 and 4000000 tetrahedral and prismatic elements). The sample surface mesh at the outlet for the mesh with 4000000 total elements number is shown in Figure 6.3, in which some mesh elements were magnified for the sake of clarity.

\subsubsection{Boundary conditions setting}

The numerical simulations were carried out with a commercial finite-volume based program, i.e., CFX 11 from Ansys INC. (Canonsburg, PA). The following conditions were assumed before computation for sake of simplification:

(1) steady inhalation flow of pure air;

(2) incompressible, isothermal air $25^{\circ} \mathrm{C}$ dry, steady-state;

(3) constant geometry with hydraulically smooth walls (no vasomotor changes);

(4) no nasal hair or mucous membranes;

(5) one-way coupling approach for the particulate phase, because a dilute suspension was used in the experiments.

\subsubsection{Boundary conditions setting for RANS/EIM approach}

The basic boundary conditions for RANS/EIM approach were explained in the following paragraphs. 
Inlet: Five different volume flow rates were chosen in performing the simulation: $90 \mathrm{~L} / \mathrm{min}, 60 \mathrm{~L} / \mathrm{min}, 45 \mathrm{~L} / \mathrm{min}, 30 \mathrm{~L} / \mathrm{min}$, and $15 \mathrm{~L} / \mathrm{min}$. For the $k-\varepsilon$ turbulence model and Reynolds stress models, the inlet turbulence quantities, $k$ and $\varepsilon$, were either specified directly or calculated using expressions that scale the distribution at the inlet according to the turbulence intensity, $I$, where:

$$
I=\frac{u}{U}
$$

There are several options to set the fluid values for the calculation domain when using CFX software, such as: turbulence intensity and length scale, intensity and eddy viscosity ratio, $k$ and $\varepsilon$, or intensity and auto compute length. When the turbulence intensity and length scale are both specified, the turbulence kinetic energy and dissipation are calculated using Equation 6.27 and Equation 6.28:

$$
k_{\text {inlet }}=\frac{3}{2} I^{2} U^{2}
$$

and

$$
\varepsilon_{\text {inlet }}=\frac{k_{\text {inlet }}^{\frac{3}{2}}}{l_{t}}
$$

where $l_{t}$ is the specified length scale at the inlet.

The inlet flow was defined as a constant volume flow rate and a turbulence intensity of $10 \%$ (for high flow rate) or $5 \%$ (for lower flow rate) of the mean velocity, and a turbulence length scale of $10 \%$ of the inlet hydraulic diameter was used at this location. To find out the effect of turbulence level to motion of particle, airflow with $1 \%$ and $10 \%$ turbulence intensities were also simulated. Table 6.2 shows the mass flow rate, velocities 
and Reynolds numbers at the inlet (calculated using the hydraulic diameter of the nasal cavity inlet plane) for different flow rates.

Table 6.2 Velocities and Reynolds numbers for different flow rates

\begin{tabular}{|l|c|c|c|c|c|}
\hline Flow rate $(\mathbf{l} / \mathrm{min})$ & 90 & 60 & 45 & 30 & 15 \\
\hline mass flow rate (kg/s) & 0.0018 & 0.0012 & 0.0009 & 0.0006 & 0.0003 \\
\hline Velocity $(\mathbf{m} / \mathbf{s})$ & 18.048 & 12.032 & 9.024 & 6.016 & 3.008 \\
\hline Re & 9821 & 6547 & 4910 & 3274 & 1637 \\
\hline
\end{tabular}

Outlet: When using RANS, the outflow condition of a constant pressure of $0 \mathrm{~Pa}$ was applied at a location corresponding to the outlet opening of the nasal models.

Walls: As previously mentioned, in present simulations the wall of the nasal cavity model was assumed as hydraulically smooth, no slip wall without vasomotor changes.

Particle setting: In present simulations, many particles $(10,000$ of each particle diameter) with various diameters (ranging from 0.29 to and $14.14 \mu \mathrm{m}$ ) and density $\rho_{p}=912 \mathrm{~kg} / \mathrm{m}^{3}$ are released in the calculated flow domain, and particle deposition positions are recorded. In order to keep the same inertial impaction parameter, $d_{a}^{2} Q$, for comparison purposes, different particle sizes were used for particle tracking simulations involving different inhalation flow rates as described in Table 6.3. Here $d_{p}$ is the particle diameter in $\mu m$ and $\mathrm{Q}$ is the inhalation flow rate in $\mathrm{L} / \mathrm{min}$. Initial particle velocities were set equal to the fluid velocity. Since top hat profiles are used as inlet conditions, particles are randomly seeded at the circular inlet with an even spatial concentration distribution.

Particle tracking can be performed with and without turbulent dispersion. In the CFX 11.0 environment, it is controlled by setting the turbulent dispersion force as "on" or "off". The turbulent dispersion force also can be controlled flexibly to be active in 
regions where the turbulent eddy viscosity ratio is above the specified Eddy Viscosity Ratio Limit. Eddy viscosity ratio $\frac{\mu_{t}}{\mu}$ represents the ratio between turbulent viscosity and the dynamic viscosity of the fluid. The default value of the Eddy Viscosity Ratio Limit is 5. In the region where the eddy viscosity ratio is above the user specified limit, the flow is assumed to be turbulent, and particle tracking is performed with turbulent dispersion. Conversely, if the ratio is smaller than the limit, then the flow can be considered as laminar and no turbulent dispersion is used, thus the particle tracking is called "laminar tracking".

There are two very important coefficients for particles in the wall boundaries setting: parallel and perpendicular restitution coefficients, which describe the actions of particles when they hit a wall. Coefficient values of 1 describe an elastic collision, while values less than 1 describe an inelastic collision. In present simulation, both coefficients were set to zero, which indicate the tracking of particles terminate when they hit the wall of nasal cavity.

Table 6.3 Physical particle diameters, $d_{p}(\mu m)$, used in simulations for different values of the impaction parameter and flow rate

\begin{tabular}{|c|c|c|c|c|c|c|c|c|c|}
\hline \multirow{2}{*}{$\begin{array}{c}\text { Flow rate } \\
(\text { L/min) }\end{array}$} & \multicolumn{8}{|c|}{ Impaction parameter $\left(\boldsymbol{\mu m}^{\mathbf{2}} \mathbf{L} / \mathbf{m i n}\right)$} \\
\cline { 2 - 11 } & 6.84 & 27.36 & 109.4 & 246.2 & 437.8 & 684.0 & 985.0 & 1751 & 2736 \\
\hline 15 & 0.71 & 1.41 & 2.83 & 4.24 & 5.66 & 7.07 & 8.49 & 11.31 & 14.14 \\
\hline 30 & 0.50 & 1.00 & 2.00 & 3.00 & 4.00 & 5.00 & 6.00 & 8.00 & 10.00 \\
\hline 45 & 0.41 & 0.82 & 1.63 & 2.45 & 3.27 & 4.08 & 4.90 & 6.53 & 8.16 \\
\hline 60 & 0.35 & 0.71 & 1.41 & 2.12 & 2.83 & 3.54 & 4.24 & 5.66 & 7.07 \\
\hline 90 & 0.29 & 0.58 & 1.15 & 1.73 & 2.31 & 2.89 & 3.46 & 4.62 & 5.77 \\
\hline
\end{tabular}




\subsubsection{Boundary conditions and solve setting for LES}

Inlet: For the inlet condition of the present LES simulation, a top hat profile giving steady flow rates of 30,60 and $90 \mathrm{~L} / \mathrm{min}$ was used (no fluctuations were added).

Outlet: For the outlet, a zero pressure gage opening boundary condition was applied.

Initialization: The present simulation is initialized from a converged RANS velocity field as described in the previous section. No forcing is added on the computational domain. In order to eliminate the effect of initial conditions, 2000 initial time steps were used. An additional 24,000 time steps were used in the particle deposition simulation.

Time advancement stability and accuracy were assured by adopting a time step that gave a CFL (Courant-Friedrich-Levy) number smaller than 1 throughout the computational domain, requiring a time step of typically $1.0 \mu \mathrm{s}$. A central differencing scheme was used in the LES computations. For the time advancement, a second-order accurate implementation using a backward implicit difference formula was used. Particles were released in the frozen computational domain and deposition statistics were gathered in a single iteration using a steady mode without any model and $1 \mu s$ of physical timescale in the convergence control (see CFX 11 manual). This procedure was adopted because the dynamic particle tracking was not available in CFX 11 for the unsteady LES mode. Calculations were performed on a Pentium IV cluster having 20 nodes, requiring approximately $250 \mathrm{CPU}$ hours, which shows that LES is considerably more expensive when compared with a standard RANS/EIM approach. 


\section{Chapter 7: $\quad$ Simulation Results}

\subsection{Grid convergence analysis}

The importance of the assessment of the numerical uncertainty in CFD calculations has been highlighted by several authors (for example, Roache, 1998; Freitas, 2002). Uncertainty is defined as, "A potential deficiency in any phase or activity of the modeling process that is due to the lack of knowledge." (AIAA G-077-1998).

In the present report, a grid convergence analysis was performed using four different grid sizes (approximately $300000,1000000,2000000$, and 4000000 tetra and prism elements as previously stated) based on the RANS simulations results. Pressure drop through the nasal cavity, velocity distribution at certain locations of the geometry and total particle deposition through the whole nasal cavity model were chosen as verification parameters. It should be noted that grid convergence analysis in LES is not as straightforward, since one may refine the grid towards DNS resolution.

\subsubsection{Pressure drop}

Figure 7.1 illustrates the calculated pressure drop through the nasal cavity for 4000000 elements compared with the experimental data and experimental tolerance obtained by Weinhold et al. (2004). Although Weinhold's replica was different from the present standardized nasal geometry, simulation results are within the estimated tolerance 
of the measured data for several flow rates ranging from 15 to $60 \mathrm{~L} / \mathrm{min}$, indicating a good qualitative agreement.

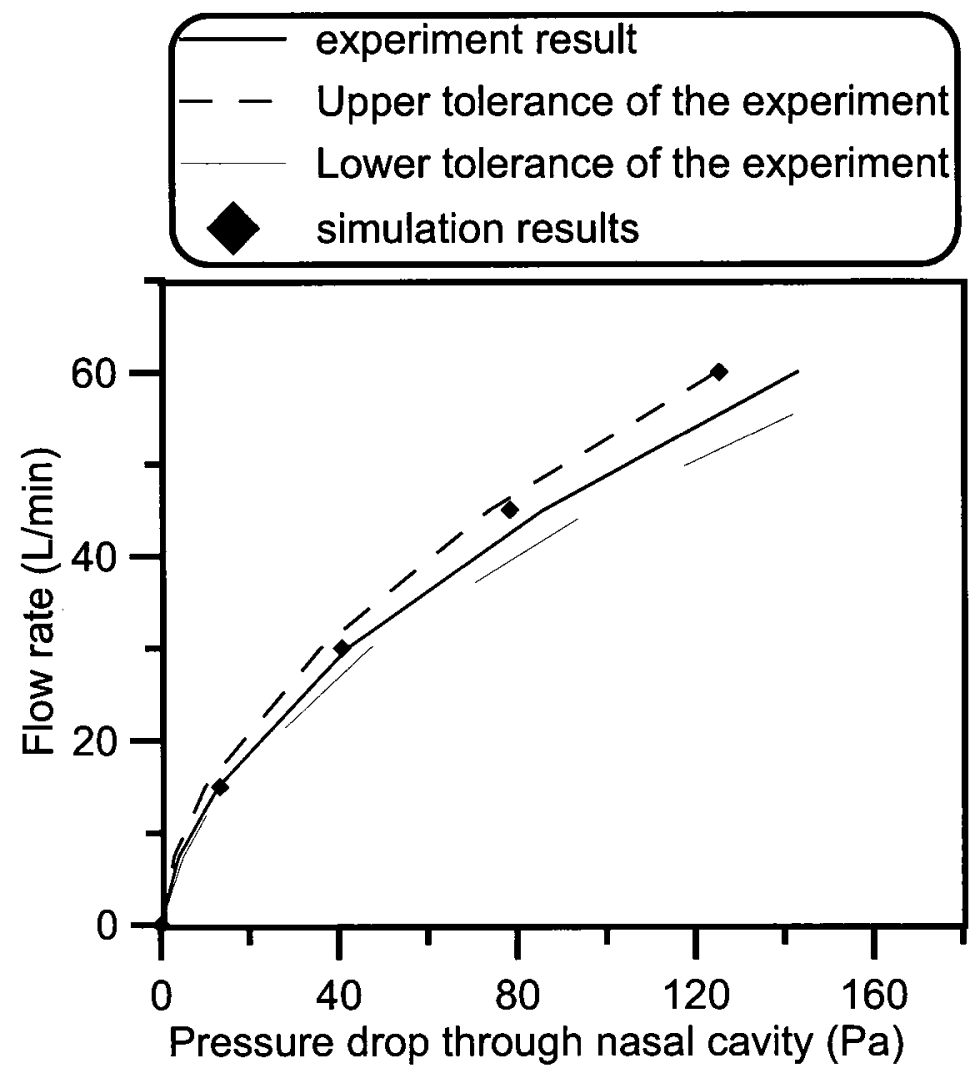

Figure 7.1 Simulation results of the pressure drop through nasal cavity compared with experimental results (Weinhold et al., 2004)

\subsubsection{Velocity profile at arbitrary location}

The velocity profile over an arbitrary line at a cross section $66 \mathrm{~mm}$ posterior to the nostril for a constant inhalation flow rate of $30 \mathrm{~L} / \mathrm{min}$ is shown separately in Figure 7.2 for different grid sizes. For higher grid sizes (2 000000 , and 4000000 elements), the profiles of each line look very similar indicating a good grid size adequacy; however, there still are some differences between the profiles of velocity distribution of 2000000 , and 4000000 elements. This is not unexpected, and it is conjectured that the differences 
in velocities are due mainly to the irregular unstructured grid meshing and the high complexity of the nasal cavity.

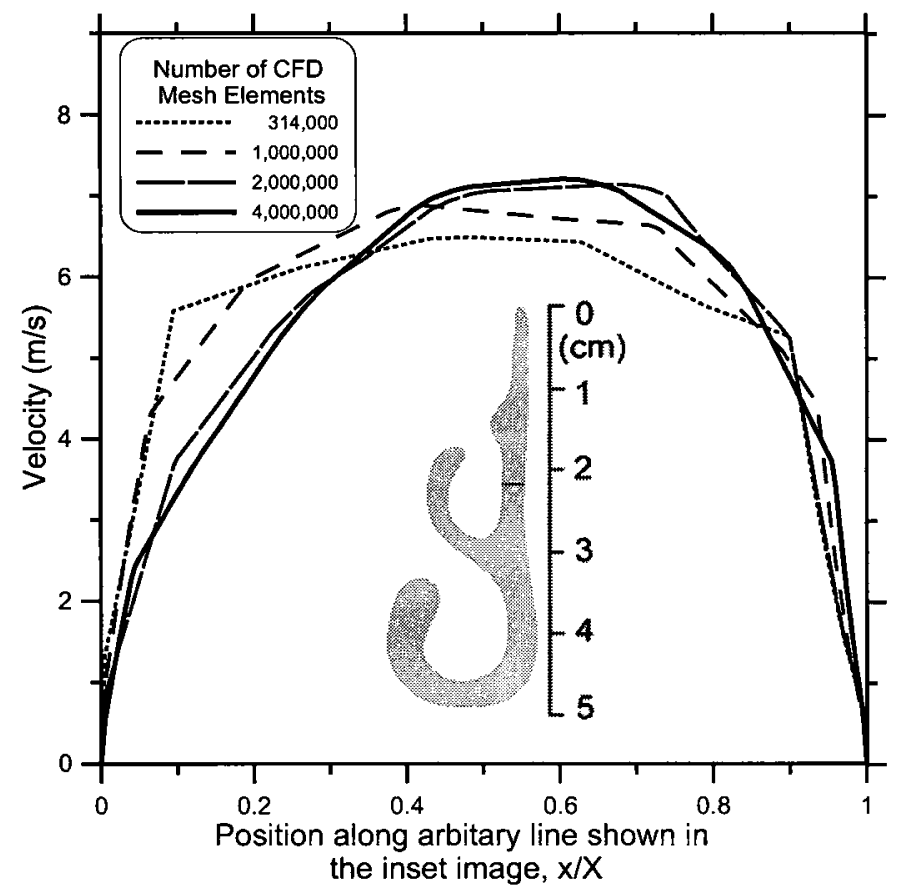

Figure 7.2 Grid size effect on velocity distribution on arbitrary line, which is on the cross section cross section $66 \mathrm{~mm}$ posterior to the nostril (flow rate $=30 \mathrm{~L} / \mathrm{min}$ )

\subsubsection{Grid effect on particle deposition fraction}

The effect of computational grid size on calculated particle deposition fraction using turbulence tracking method for the flow rate $Q=60 \mathrm{~L} / \mathrm{min}$ is illustrated in Figure 7.3. Specified particle diameters vary from $0.71 \mu \mathrm{m}$ to $7.07 \mu \mathrm{m}$. For smaller particles $(0.71$, $1.41,2.12$ and $2.83 \mu \mathrm{m}$ ), calculated deposition efficiencies decreased slightly when the grid size was increased, and efficiencies were very close in value for 2000000 and 4000000 elements. For larger particles $(3.54,4.24,5.66$, and $7.07 \mu \mathrm{m})$, the calculated results for particle deposition fraction increased slightly when the grid size was increased, 
and approached an asymptotic behaviour. These results suggest that coarse mesh may result in over-estimation of particle deposition for smaller particles, and a 4000000 element grid is sufficient for the deposition calculation.

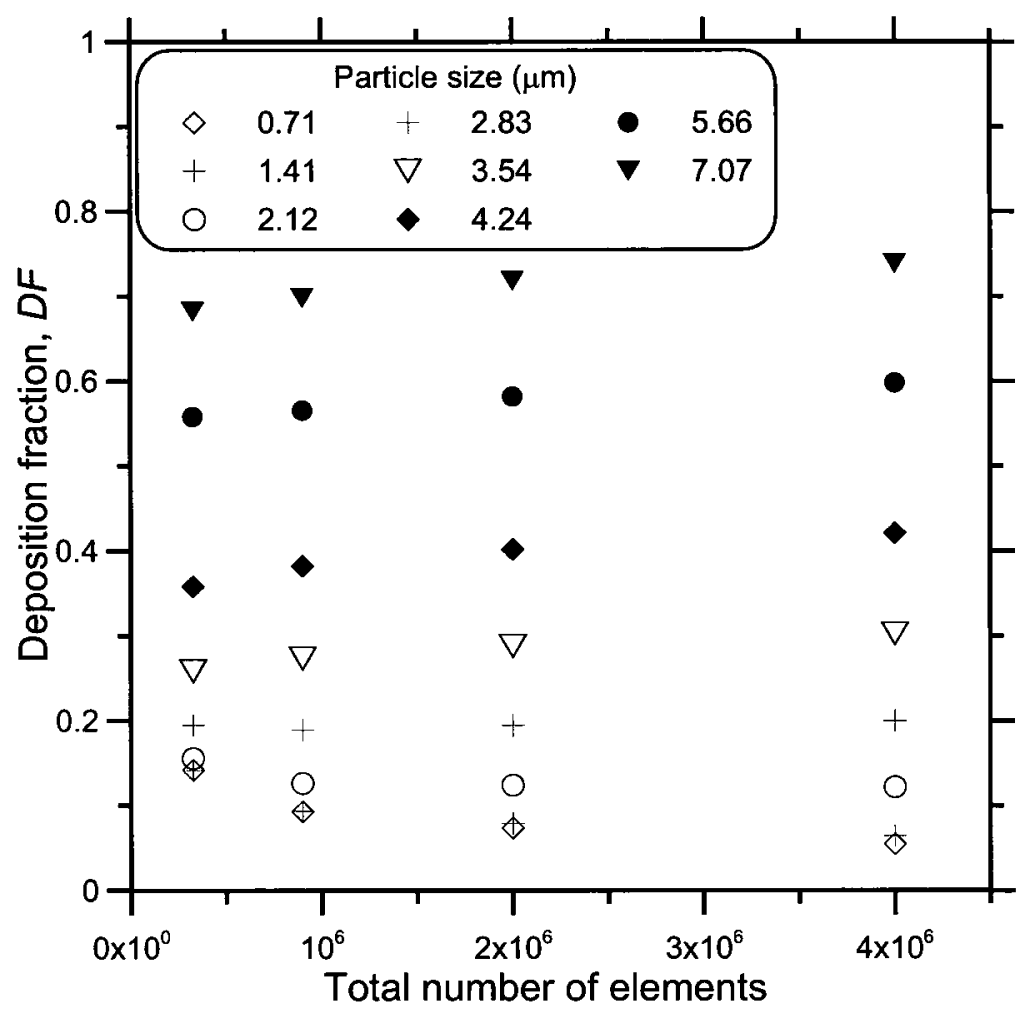

Figure 7.3 Effect of computational grid size on particle deposition fraction $(Q$ $=60 \mathrm{~L} / \mathrm{min}$ )

\subsubsection{Effect of iteration numbers on LES simulation results}

The total deposition using LES was tested using different iteration numbers (i.e., time steps) for the inhalation flow rate of $90 \mathrm{~L} / \mathrm{min}$. Figure 7.4 shows the total particle deposition using LES as a function of the number of iterations for two different particle sizes $\left(d_{p}=3.46\right.$ and $\left.d_{p}=1.73 \mu \mathrm{m}\right)$. It can be seen that the total deposition fraction $(D F)$ decreases when the iteration number is increased, and the relationship between these two 
variables shows an asymptotic trend. After $1.5 \times 10^{4}$ time iterations, total particle deposition fraction oscillates about an average value $\left(D F=0.44\right.$ for $d_{p}=3.46$ and $\left(D F=0.12\right.$ for $\left.d_{p}=1.73\right)$.

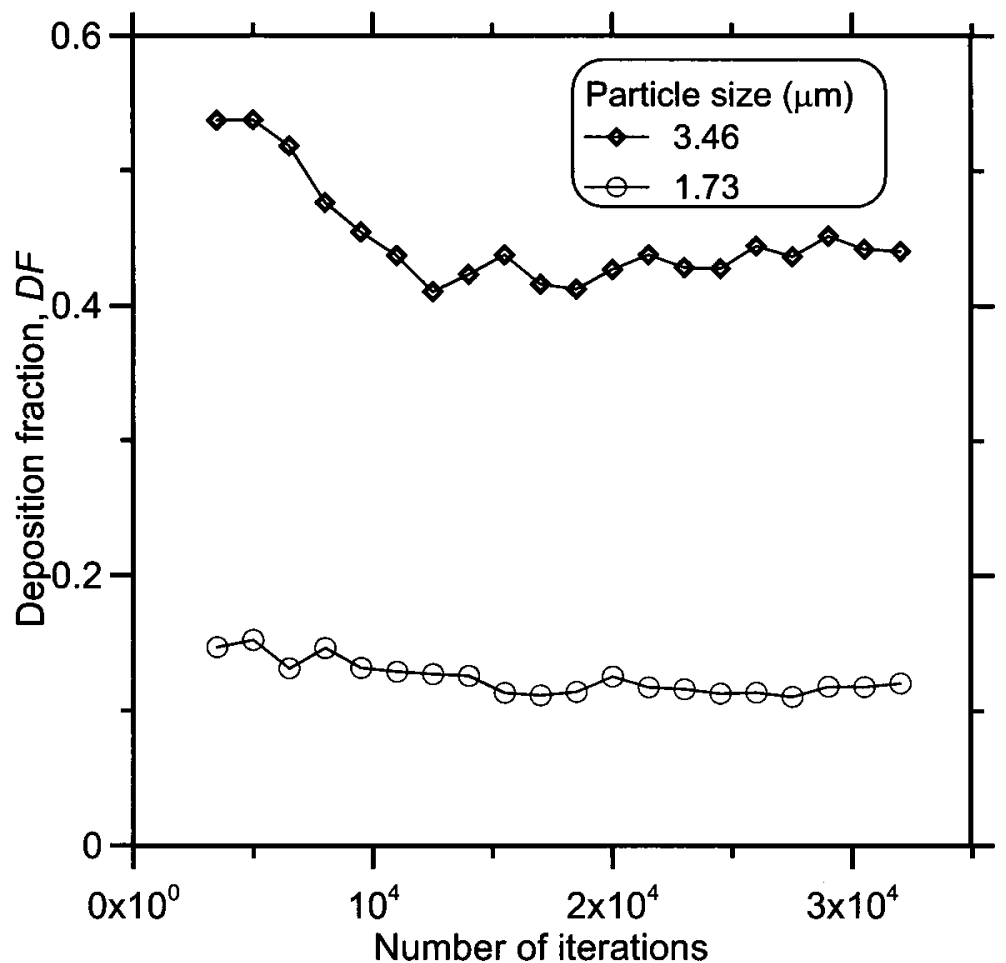

Figure 7.4 LES results of total particle deposition fraction as function of number of iterations

\subsection{Sensitivity analysis}

As mentioned in section 4.3.2, when using the RANS/EIM approach, the turbulence intensity and length scale are both specified for inlet boundary condition. There are several other fluid values that can be set, apart from turbulence intensity and length scale, for the calculation domain when using CFX software, such as: intensity and eddy viscosity ratio, $k$ and epsilon, or intensity and auto compute length. Based on 
fundamental of fluid dynamics, when the turbulence intensity and length scale are both specified for inlet boundary condition, the inlet turbulent kinetic energy, $k$, dissipation of turbulence kinetic energy, $\varepsilon$, eddy viscosity ratio, $\frac{\mu_{T}}{\mu}$, are also set.

The effect of fractional turbulence intensity $I$ at the inlet on particle deposition fraction, with flow rate $\mathrm{Q}=60 \mathrm{~L} / \mathrm{min}$ and inlet length scale kept constant $(10 \%$ of the hydraulic diameter of inlet), was investigated. The parameters for this investigation were given in Table 7.1.

Table 7.1 Calculation parameters for sensitivity analysis (constant inlet length scale)

\begin{tabular}{|l|l|l|l|l|l|l|l|}
\hline $\begin{array}{l}\text { Flow rate } \\
(\mathbf{L} / \mathbf{m i n})\end{array}$ & $\begin{array}{l}\text { Velocity } \\
(\mathbf{m} / \mathbf{s})\end{array}$ & $\begin{array}{l}\text { length scale } \\
(\mathbf{m})\end{array}$ & $\begin{array}{l}\text { length } \\
\text { scale ratio }\end{array}$ & $\begin{array}{l}\text { turbulence } \\
\text { intensity } \boldsymbol{l}\end{array}$ & $\begin{array}{l}\boldsymbol{k} \\
\left(\mathbf{m}^{2} / \mathbf{s}^{2}\right)\end{array}$ & $\begin{array}{l}{[\boldsymbol{E}} \\
\left(\mathbf{m}^{2} / \mathbf{s}^{3}\right)\end{array}$ & $\begin{array}{l}\text { viscosity } \\
\text { ratio }\end{array}$ \\
\hline 60 & 12.03 & 0.0008 & 0.1 & 0.01 & 0.021 & 2.612954 & 0.888 \\
\hline 60 & 12.03 & 0.0008 & 0.1 & 0.05 & 0.543 & 326.6193 & 4.4388 \\
\hline 60 & 12.03 & 0.0008 & 0.1 & 0.1 & 2.172 & 2612.954 & 8.8768 \\
\hline 60 & 12.03 & 0.0008 & 0.1 & 0.15 & 4.886 & 8818.72 & 13.3148 \\
\hline
\end{tabular}

It can been seen from the Figure 7.5 that when length scale at the inlet is kept constant, the increase in fractional turbulence intensity at the inlet enhanced the particle deposition fraction within a reasonable range, which illustrates the influence of turbulence on particle deposition fraction in the human nasal cavity. 


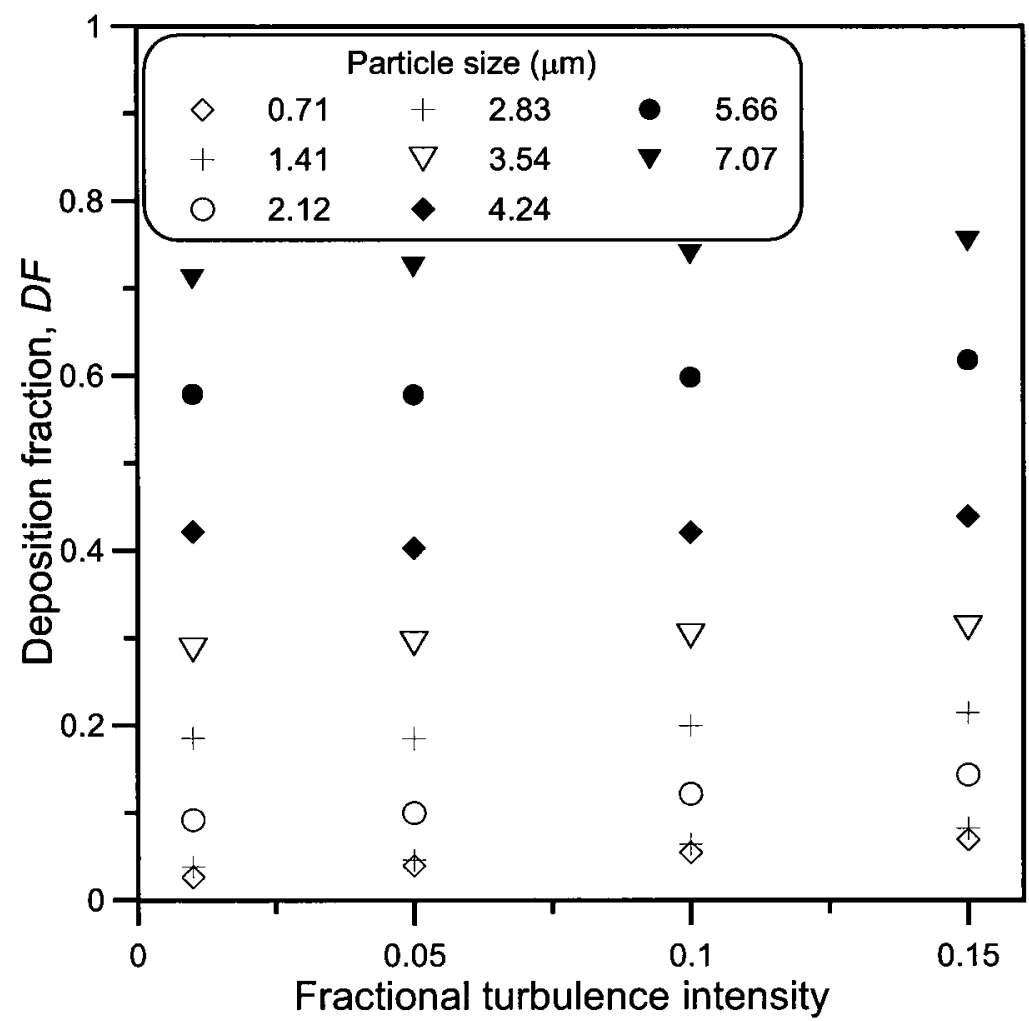

Figure 7.5 Effect of fractional turbulence intensity $I$ on particle deposition fraction $\left(Q=60 \mathrm{~L} / \mathrm{min}\right.$, length scale at inlet $\left.=D_{h} / 10\right)$

Similarly, the effect of inlet length scale ratio on particle deposition fraction, with flow rate $\mathrm{Q}=60 \mathrm{~L} / \mathrm{min}$ and constant fractional turbulence intensity $(I=0.1)$, was investigated using the parameters shown in Table 7.2.

Table 7.2 Calculation parameters for sensitivity analysis (constant inlet length scale)

\begin{tabular}{|l|l|l|l|l|l|l|l|}
\hline $\begin{array}{l}\text { Flow rate } \\
(\mathbf{L} / \mathbf{m i n})\end{array}$ & $\begin{array}{l}\text { Velocity } \\
(\mathbf{m} / \mathbf{s})\end{array}$ & $\begin{array}{l}\text { turbulence } \\
\text { intensity } \mathbf{I}\end{array}$ & $\begin{array}{l}\text { length scale } \\
(\mathbf{m})\end{array}$ & $\begin{array}{l}\text { length } \\
\text { scale ratio }\end{array}$ & $\begin{array}{l}\boldsymbol{k} \\
\left(\mathbf{m}^{2} / \mathbf{s}^{2}\right)\end{array}$ & $\begin{array}{l}\boldsymbol{\varepsilon} \\
\left(\mathbf{m}^{2} / \mathbf{s}^{3}\right)\end{array}$ & $\begin{array}{l}\text { viscosity } \\
\text { ratio }\end{array}$ \\
\hline 60 & 12.03 & 0.1 & 0.0004 & 0.05 & 2.172 & 5225.908 & 4.438 \\
\hline 60 & 12.03 & 0.1 & 0.0008 & 0.1 & 2.172 & 2612.954 & 8.876 \\
\hline 60 & 12.03 & 0.1 & 0.0016 & 0.2 & 2.172 & 1306.477 & 17.753 \\
\hline 60 & 12.03 & 0.1 & 0.004 & 0.5 & 2.172 & 522.591 & 44.381 \\
\hline
\end{tabular}

The results of this testing are shown in Figure 7.6. It can be seen that when the length scale ratio changes from 0.05 to 0.2 , the results of nasal particle deposition were 
almost constant, indicating that the chosen length for the inlet boundary $(10 \%$ of hydraulic diameter) is reasonable.

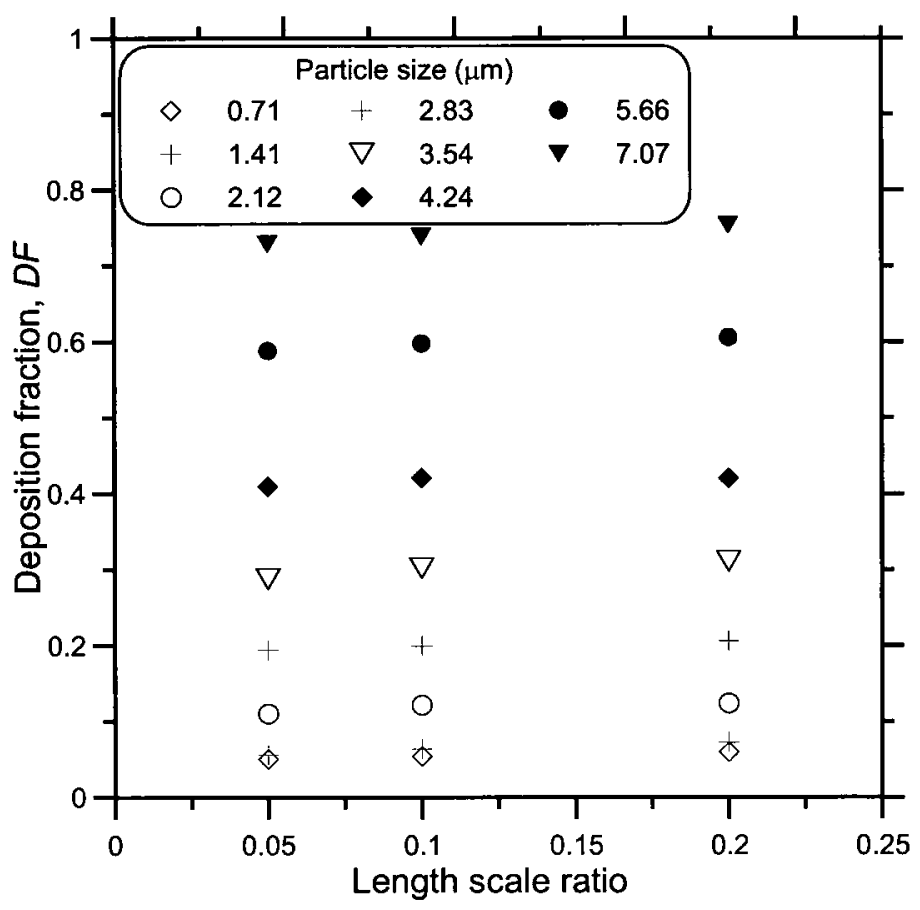

Figure 7.6 Effect of inlet length scale ratio on particle deposition fraction $(Q=60$ $\mathrm{L} / \mathrm{min}$, fractional turbulence intensity $I=0.1$ )

\subsection{Airflow structure}

\subsubsection{Velocity and kinetic energy}

\subsubsection{Distribution of velocity and turbulence kinetic energy}

Typical air flow characteristics, shown here for the inhalation flow rate of $60 \mathrm{~L} / \mathrm{min}$, can be seen from the distribution of velocity (Figure 7.7a) and turbulence kinetic energy (Figure $7.7 \mathrm{~b}$ ), at the inlet and outlet, and along 10 coronal planes of the novel standardized nasal airway model. 


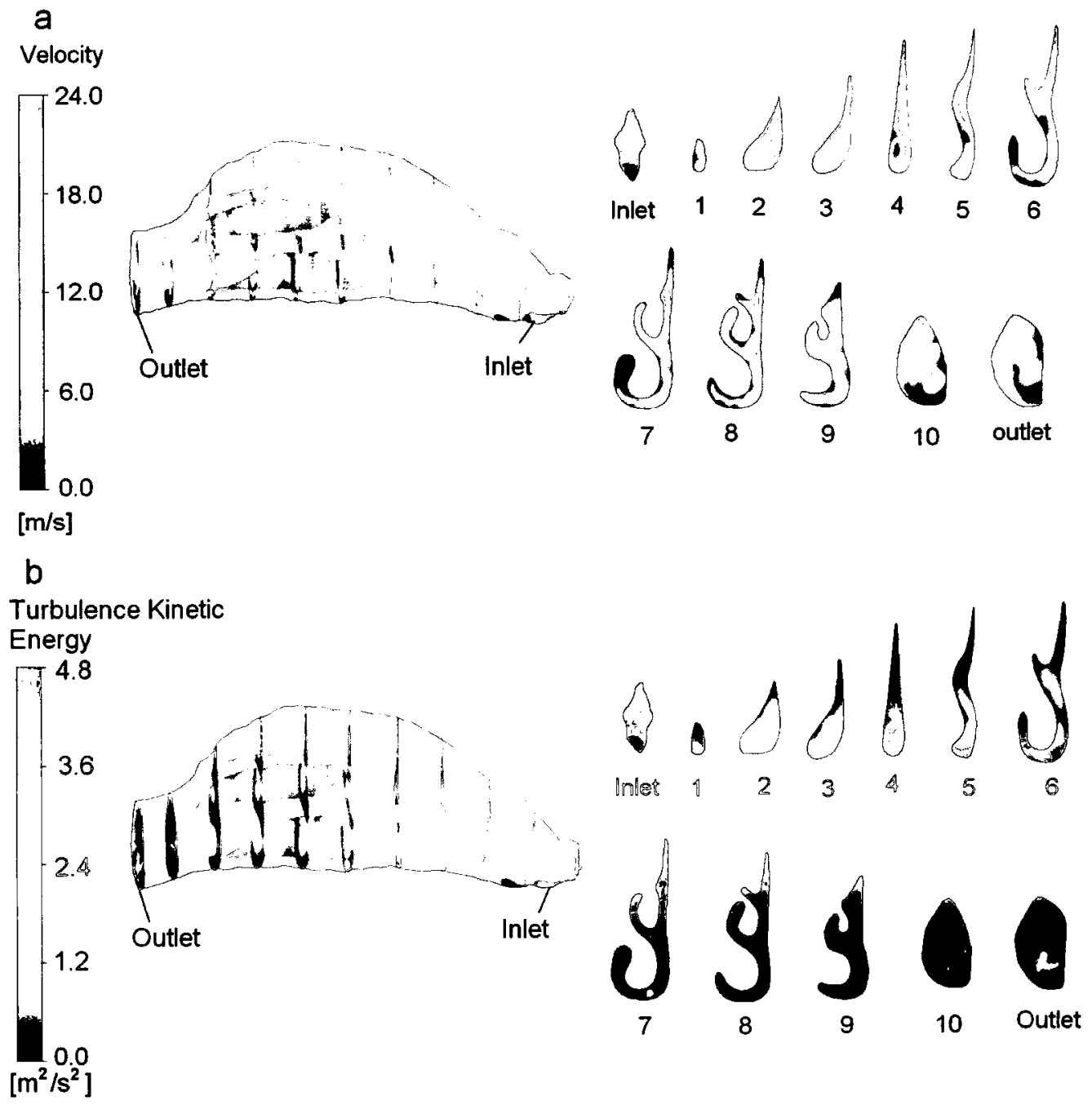

Figure 7.7 Velocity distribution (a) and turbulence kinetic energy distribution (b) in standardized human nasal cavity along coronal planes (flow rate, $Q=60 \mathrm{~L} / \mathrm{min}, 4$ 000000 elements)

Air enters the nasal cavity from the nostril and its mean velocity changes as it flows through the nasal cavity due to the change in cross-sectional area. The air impinges and circumvents the nasal valve region and the three turbinates, which have the most complex geometry of any structure in the nasal cavity (see Figure 1.1 for reference), and generates some turbulence kinetic energy and leaves the nasal cavity through the outlet. Observing the velocity distribution in one cross-sectional plane (Figure $7.7 \mathrm{a}$ ), velocities at the 
superior airway, middle meatus, and inferior airway are much higher than in other regions, which can be attributed to the smaller flow cross-sectional areas and downstream flow patterns in these regions. After passing through the nasal valve, the air is accelerated and a small amount of turbulence kinetic energy is generated (see Figure $7.7 \mathrm{~b}$ plane 4 for reference). However, this turbulent energy quickly dissipates, and after plane 6 it drops to near zero, which indicates that the downstream flow appears to be essentially laminar.

\subsubsection{Streamwise distribution of mean velocity magnitude}

The streamwise distribution of mean velocity, averaged over the local cross-sectional area, in the standardized human nasal cavity is shown in Figure 7.8 for inhalation flow rates of 30,45 and $60 \mathrm{~L} / \mathrm{min}$. The same inlet boundary conditions (i.e., $5 \%$ of the mean velocity and a turbulence length scale of $10 \%$ of the inlet hydraulic diameter) were used to study the streamwise distribution of mean velocity for three different flow rates as were used in the sensitivity analysis.

It can be seen that the mean velocities of the cross-section planes for three different inhalation flow rates share similar trends due to change in cross-sectional area (the reference for the cross-sectional area of the novel standardized nasal airway geometry is shown in Figure 3.13). The bigger the cross-sectional area of the cross-section plane, the smaller the magnitude of the mean velocity becomes. The peak value of the mean velocity appears at a dimensionless distance from the nostril equal to 0.25 , coinciding with the location of smallest cross-sectional area, which is coinciding with the location of the nasal valve. 


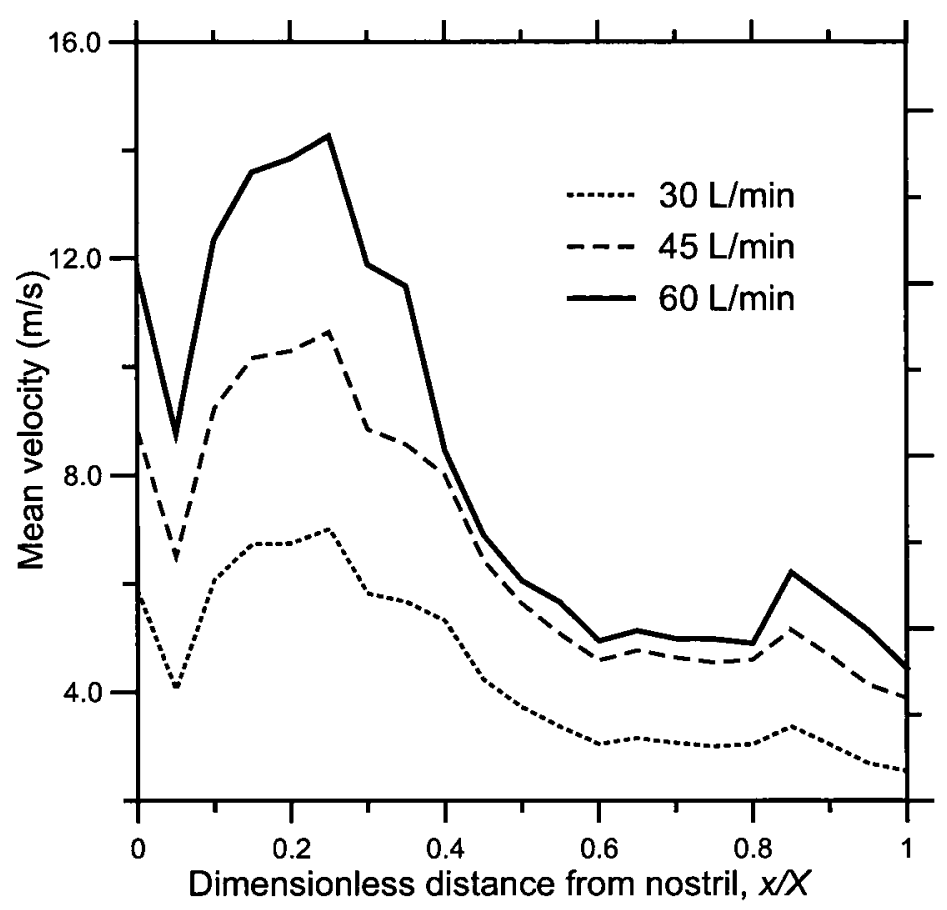

Figure 7.8 Streamwise distribution of mean velocity magnitude

\subsubsection{Streamwise distribution of turbulence kinetic energy}

The streamwise distribution of turbulence kinetic energy, $k$, averaged over the local cross-sectional area is shown in Figure 7.9 for inhalation flow rates of $45 \mathrm{~L} / \mathrm{min}$ and $60 \mathrm{~L} / \mathrm{min}$. The inlet boundary conditions for the turbulence kinetic energy measurements were $1 \%, 5 \%$ and $10 \%$ of the mean inlet velocity. For each flow rate, results for different turbulence intensities were sensitive to the inlet conditions, meaning higher values of turbulence kinetic energy intensity at the inlet resulted in higher values throughout the domain. Despite the different levels of turbulence at the inlet, the profiles of mean turbulence kinetic energy along the standardized human nasal cavity for each flow rate share similar trends, and they reach a small peak value at a dimensionless 
distance from the nostril equal to 0.35 (coinciding with the beginning and the frontal location of the turbinates).
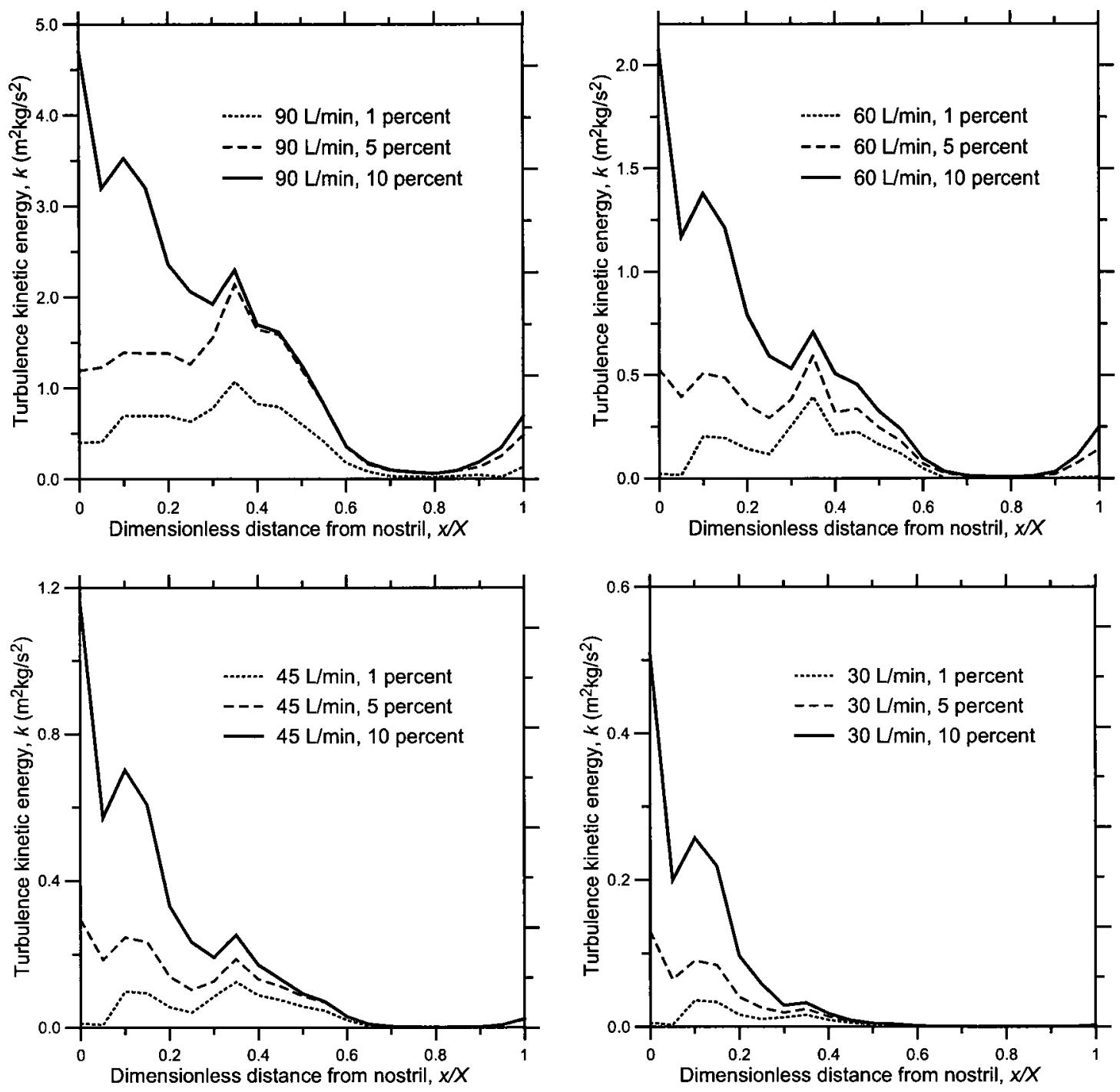

Figure 7.9 Average cross-stream turbulence kinetic energy for low (1\%), middle (5\%) and high $(10 \%)$ turbulence intensities at inlet

Table 7.3 summarizes the peak values of turbulence kinetic energy for each flow rate for high (10\%), middle (5\%) and lower (1\%) turbulence intensities. The data in Table 7.3 indicate that the turbulence models are necessary in the simulation at these inhalation 
flow rates. Beyond a dimensionless distance from the nostril of about 0.6 , the turbulence kinetic energy drops to near zero and the flow in the domain can be considered to be laminar in nature. It also can been seen that near the outlet boundary, some turbulence kinetic energy is generated, caused by the impaction of air on the wall after it leaves the three turbinates.

Table 7.3 The values of turbulence kinetic energy at the small peak for low, middle and high turbulence intensities at the inlet

\begin{tabular}{|c|c|c|c|}
\hline $\begin{array}{c}\text { Flow rate } \\
(\mathrm{L} / \mathrm{min})\end{array}$ & $\begin{array}{c}\text { Turbulence kinetic } \\
\text { energy for low } \\
\text { turbulence intensity, 1\% } \\
\left(\mathbf{m}^{2} \mathbf{k g} / \mathbf{s}^{2}\right)\end{array}$ & $\begin{array}{c}\text { Turbulence kinetic } \\
\text { energy for middle } \\
\text { turbulence intensity, 5\% } \\
\left(\mathbf{m}^{2} \mathbf{~ k g} / \mathbf{s}^{2}\right)\end{array}$ & $\begin{array}{c}\text { Turbulence kinetic } \\
\text { energy for high }\end{array}$ \\
\hline 90 & 1.068 & 2.136 & 2.295 \\
\hline 60 & 0.395 & 0.592 & 0.707 \\
\hline 45 & 0.124 & 0.186 & 0.252 \\
\hline 30 & 0.016 & 0.024 & 0.032 \\
\hline
\end{tabular}

\subsubsection{Particle motion and deposition patterns}

Figure 7.10 shows the complex trajectories and travel distances of particles for the flow rate of $60 \mathrm{~L} / \mathrm{min}$. For illustrative purposes and clarity, only 600 of the 10000 calculated trajectories of particles are shown in each of Figure 7.10.a-c for the RANS/EIM "turbulent tracking", "mean flow tracking" and LES simulation, respectively.

It can be seen that there is a recirculation zone at the anterior region of the nasal cavity near the nostril. On close inspection, it can be seen from the "turbulent tracking" results that fewer particles reach the outlet (meaning more deposition) when compared to the "mean flow tracking" approach. The particle trajectories using LES particle tracking are similar to the turbulent tracking data, while the velocity fluctuations can be observed from LES particle trajectories. 


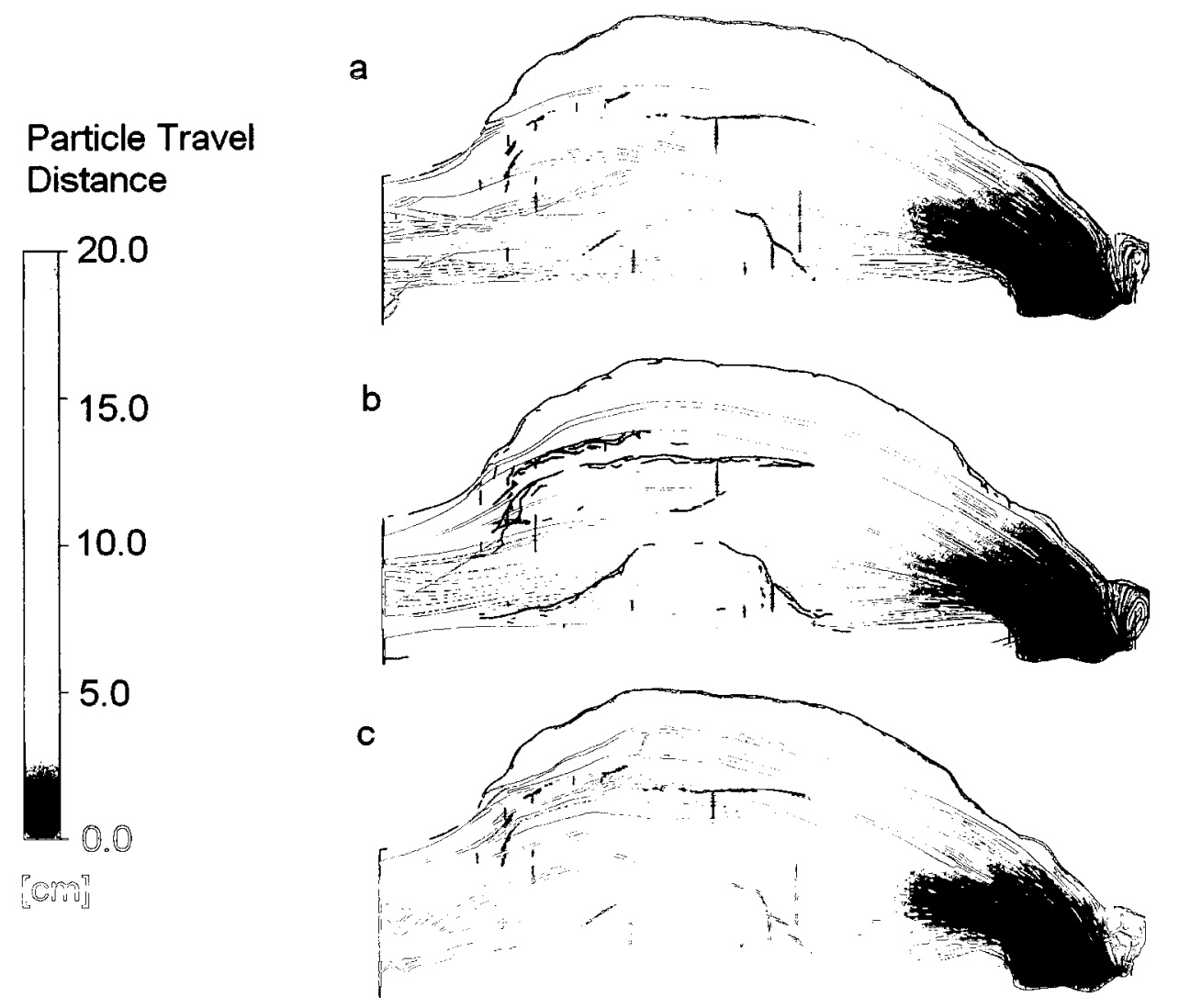

Figure 7.10 Particle tracking with particle travel distance $(Q=60 \mathrm{~L} / \mathrm{min}, \mathrm{dp}=7.071$ $\mu \mathrm{m}, \mathrm{d}_{\mathrm{p}}{ }^{2} \mathrm{Q}=$ 2736). (a) RANS/EIM turbulence tracking. (b) RANS/EIM mean flow tracking. (c) LES.

\subsubsection{Simulated results of total particle deposition fraction}

\subsubsection{Effect of different RANS model on particle deposition fraction}

Particle deposition fraction results using the SST model, standard $k-\omega$ model and standard $k-\varepsilon$ model with turbulent particle tracking are shown in Figure 7.11. Results of the SST model and the $k-\omega$ model show the same trends with a slight over-prediction for small inertial impaction parameter values and under-prediction for large inertial impaction parameter values. The results of the $k-\varepsilon$ model show significant over- 
prediction for the whole range of inertial impaction parameters and cannot reveal the important role of inertial impaction in the deposition of particles. This suggests that the standard $k-\varepsilon$ model is not appropriate for an airflow and particle deposition simulation of the geometrically complex nasal cavity. The results of the RANS/EIM approach in the present work were all obtained using the $k-\omega \mathrm{SST}$ model. They are discussed in detail in section 7.3.3.2 with present measurement results.

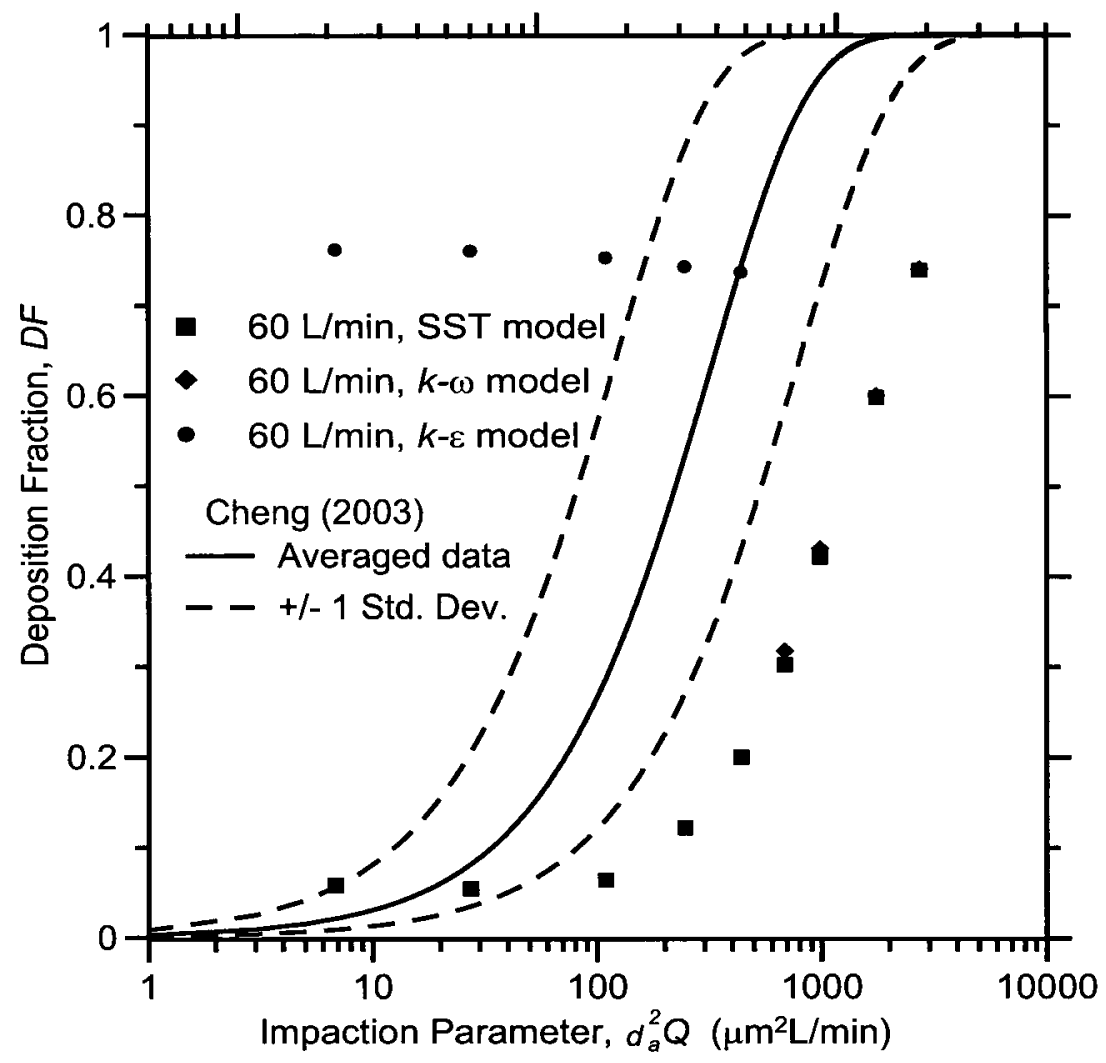

Figure 7.11 Particle deposition fraction results using different RANS model (turbulent tracking method)

\subsubsection{Simulated results of total particle deposition fraction}

Simulated deposition results using the mean flow tracking method (i.e. without turbulent 
diffusion), the turbulent tracking method (i.e. with turbulent diffusion), and LES for different flow rates $(30,60$ and $90 \mathrm{~L} / \mathrm{min}$ using the same grid resolution of 4000000 tetrahedric elements) are shown in Figure 7.12, together with data from the current experiments. For the RANS based simulations, the air flow was calculated using the SST turbulence model with turbulence intensity of $5 \%$ of the mean velocity at inlet location.

The numerical results shows good agreement when compared with the present in vitro measurements, especially for inertial impaction parameter ranged 100 800. Close inspection of the graph reveals that the deposition results using RANS simulations with either turbulent tracking (using the eddy interaction model) or mean flow tracking are quite similar, indicating that inertial impaction is the dominant mode of deposition in the nasal cavity, even for these relatively small particles.

For larger impaction parameters $\left(d_{a}^{2} Q>1000\right)$, the numerical simulations show a slight under-prediction of the present in vitro deposition data. This is likely attributable to an effect of wall roughness. In the numerical simulation, the boundary condition for the wall of the nasal cavity was specified as smooth, whereas there is a small but unavoidable wall roughness along the inner wall of the in vitro model due to manufacturing. Some of this difference might also be attributable to differences between actual inlet conditions in the experiment and those chosen for the simulations, although this effect is thought to be less significant as discussed in Figure 5.2 of section 5.1.1.2.

For smaller values of the inertial parameter $\left(d_{a}^{2} Q<200\right)$, the RANS turbulent tracking simulations corresponding to an inhalation flow rate of $90 \mathrm{~L} / \mathrm{min}$ deviated from the rest of the data. Similar errors have been observed in other simulations that have appeared in some published works (e.g., Tang et al., 2004, Liu et al., 2007), and are 
thought to be related to the linear interpolation used to determine the fluid velocity and other turbulence statistics at the particle's location. The inaccuracies in the linear interpolation arise during the integration of the particle equation of motion. Inaccuracies might arise on account of the mesh elements attached to the walls, which affect deposition of particles with a small inertial parameter.

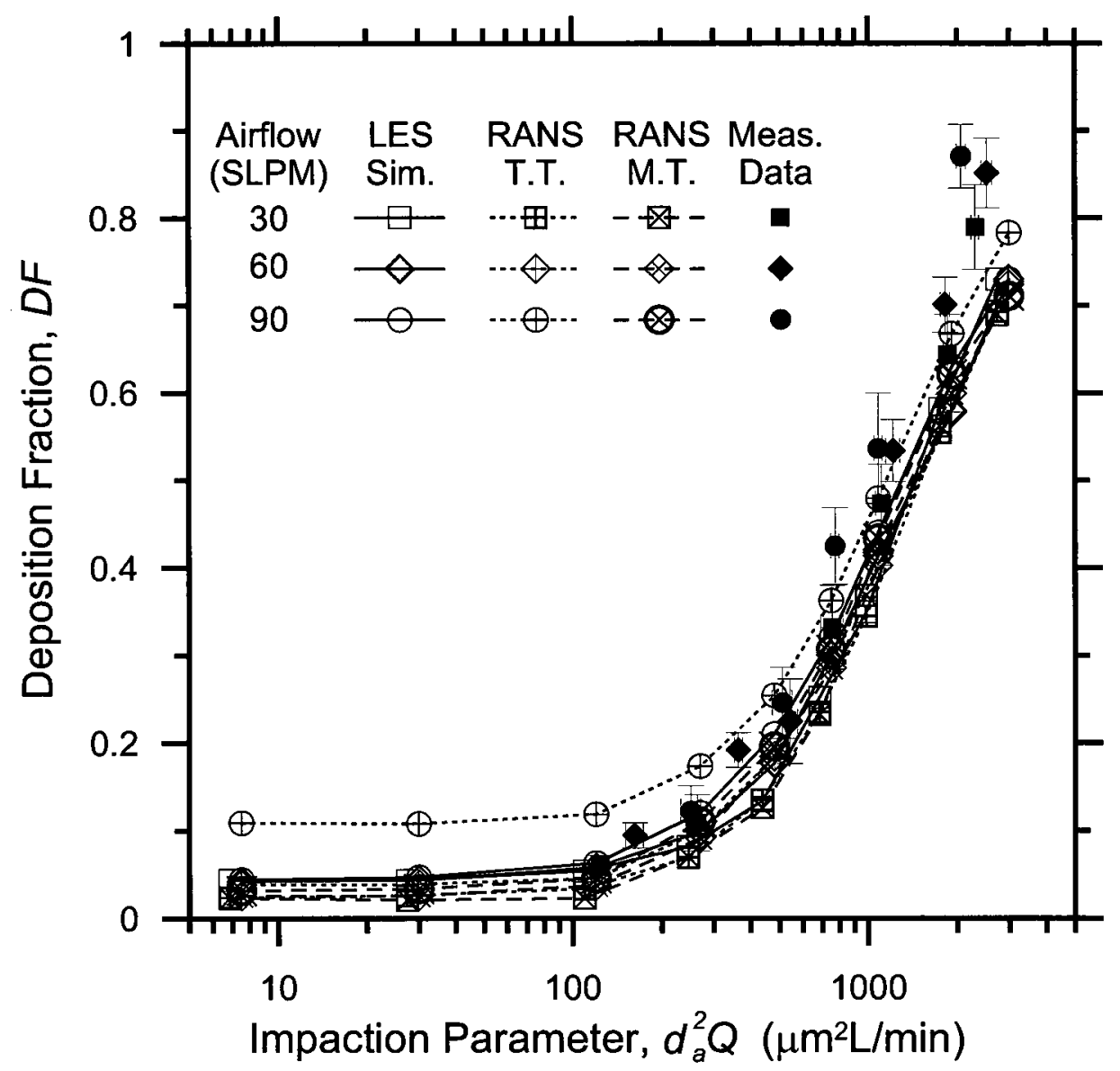

Figure 7.12 Deposition results using different simulation methods compared with measured data

In addition to different particle diameters, particles of different densities are used to analyse the influence of particle density on the deposition fraction. The deposition 
curves, created using the same tracking method, completely overlap when they are plotted as a function of the impaction parameter, no matter what the density is.

The comparison of simulation results with previous deposition simulations of several other researchers (Inthavong et al., 2006; Tian et al., 2007; Shi et al., 2007; Shanley et al., 2008) is shown in Figure 7.13. Although the nasal cavity models of cited works were generated from different subjects (Inthavong et al., 2006 and Tian et al., 2007: CT scans of a 25-year-old healthy, non-smoking Asian male; Shi et al., 2007: MRI scans of a 53year-old non-smoking Caucasian male originating from Swift (1991); Shanley et al., 2008: MRI scans of an anonymous, healthy, adult male human subject), these results show consistent trends. It can be noted that the current simulation results fall in the middle range of other published numerical predictions of particle deposition. Given that the current numerical results match the present experimental data using the same standardized nasal model as shown in Figure 7.12, this provides additional support for the discussion of differences among in vitro deposition data from different geometries presented in Section 2.3.2.2 and Section 5.1.1.2 above. Specifically, the simulations of Shi et al. (2007), using the geometry of Swift (1991) also used by the range of studies corresponding to blue symbols in Figure 7 above, span the current simulated data as the effects of wall roughness are modeled numerically. At high values of the impaction parameter, the simulations of Shanley et al. (2008) and Tian et al. (2007) also fall on either side of the current data. Although these studies considered different nasal geometries, both modeled deposition using the Lagrangian particle tracking algorithm within the commercial FLUENT CFD software for similar flow ranges and assuming laminar flow. The sigmoid trends of deposition fraction are quite similar between these 
studies and the differences in magnitude of deposition fraction at fixed values of the impaction parameter are a strong indication of the importance of inter-subject variability, which in this case spans the data for the standardized geometry.

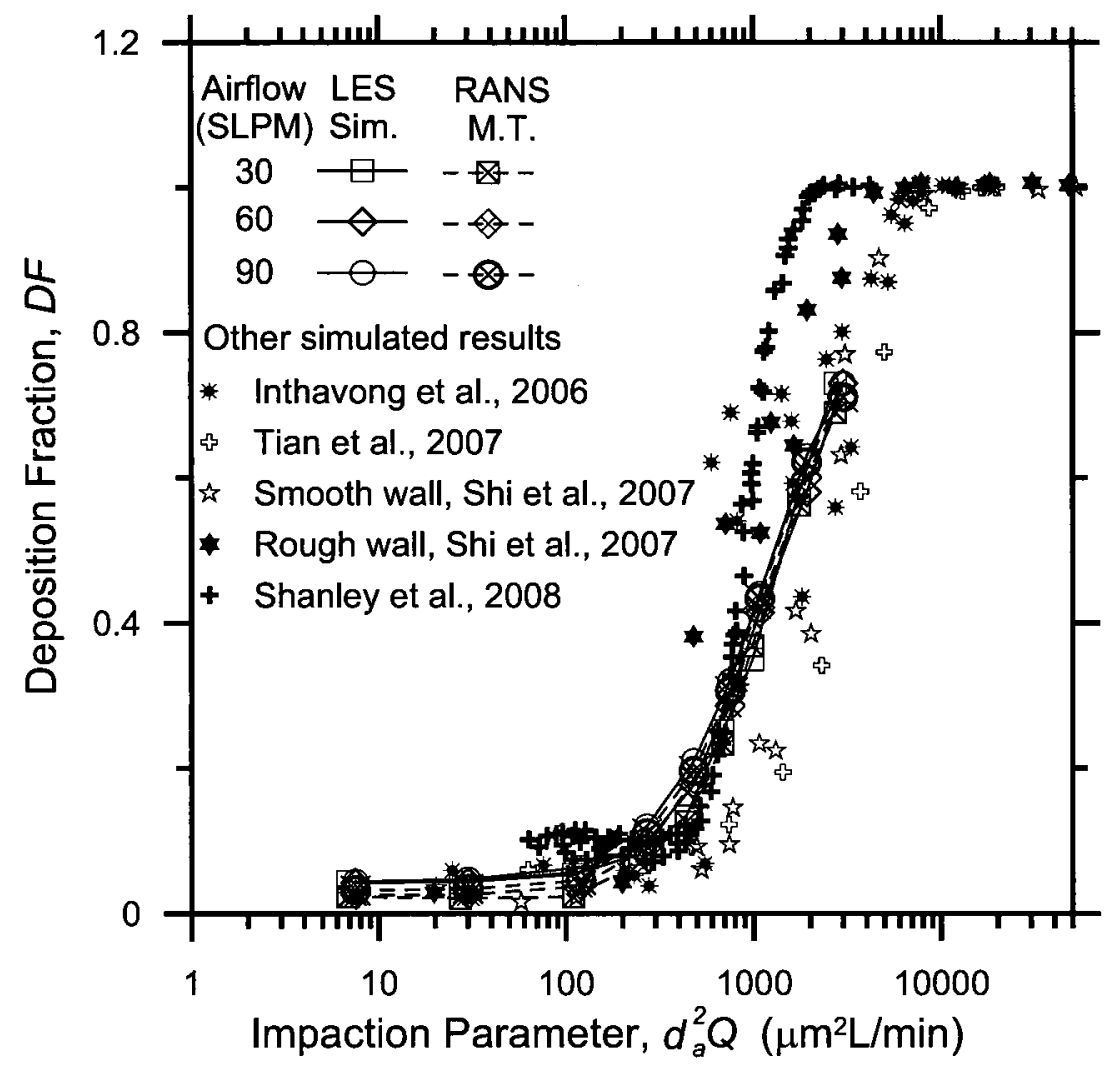

Figure 7.13 Comparison of current simulation results with published simulation data from other researchers

\subsubsection{Regional particle deposition fraction results}

\subsubsection{Simulated results of regional particle deposition fraction distribution}

A quantitative analysis of regional distribution of particle deposition within the nasal cavity was also performed in the present work. Figure 7.14.a-c illustrates the LES simulation results of regional particle deposition fraction on the surface of the nasal 
passage for inhalation flow rates of 30,60 , and $90 \mathrm{~L} / \mathrm{min}$ with a variety of different particle sizes. For this range of flow rates, the largest particles (with diameters ranging from $5.77-10.0 \mu \mathrm{m}$ ) deposit most significantly in the first $30 \%$ of the length of the nasal cavity. Moreover, in the anterior region, the majority of deposited particles impact on the cavity walls near the location of the nasal valve (i.e., at a dimensionless distance from the nostril equal to approximately 0.3 ). These results highlight the critical importance of considering the geometry in the region of the nostrils and nasal valve in determining deposition rates.

In the context of drug delivery, these results suggest that while it may be relatively easy to target deposition in the early part of the nasal cavity, it is much more difficult to deposit particles in the middle and posterior cavity without having them carry on through the nasopharynx. It is also noted by Shanley et al. (2008) through simulations that smaller particles mostly escape into the nasopharynx, while larger particles deposit preferentially in the anterior region with increasing Stokes number. Considering the fact that the turbinates and olfactory region of the nasal cavity are the locations where most of drug absorption occurs (Illum, 2000), the desirable particles sizes for drug delivery for different flow rates could be obtained by the regional distribution of the deposited particles shown in Figure 7.14. For example, at the lowest flow rate of $30 \mathrm{~L} / \mathrm{min}$ (Figure 7.14a), deposition of $10 \mu \mathrm{m}$ particles shows a bimodal distribution with a secondary peak at approximately $60 \%$ of the distance into the cavity. For smaller sized particles, there is an abrupt drop in deposition for all three flow rates. The limited deposition that occurs is also more evenly distributed throughout the nasal cavity. 

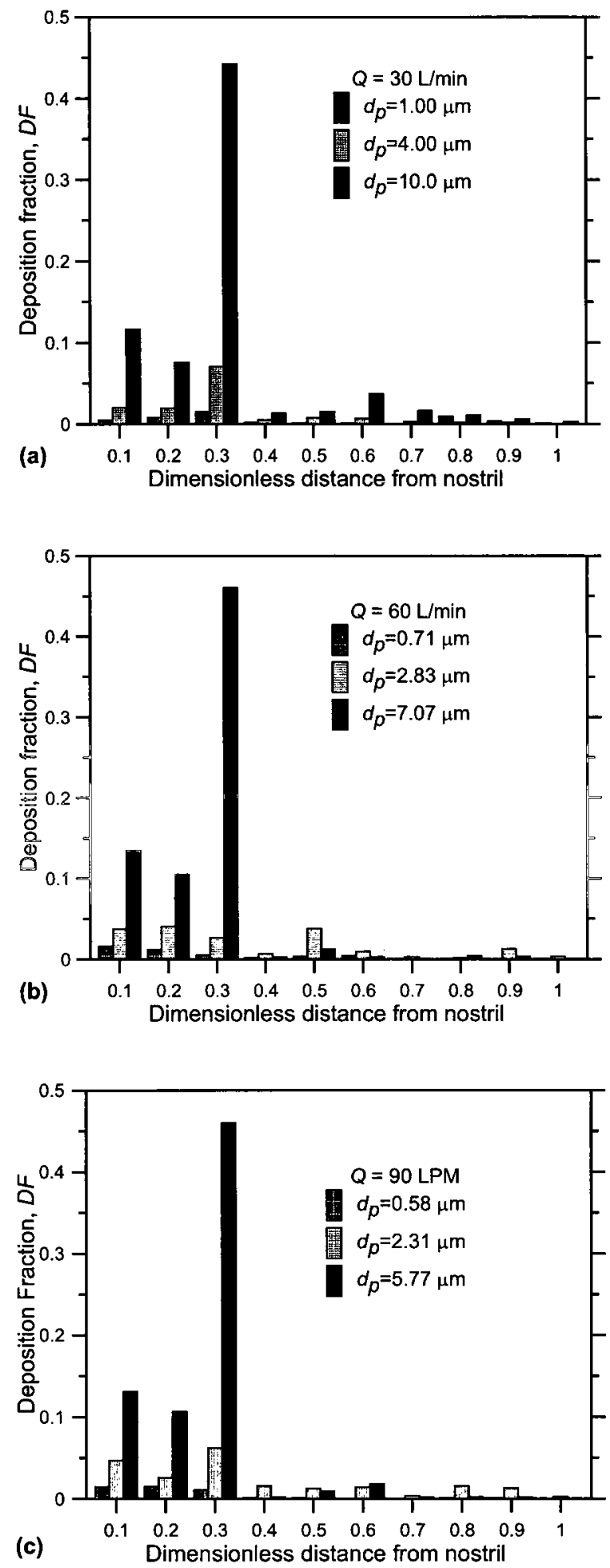

Figure 7.14 Regional particle deposition fraction for different sized particles calculated from LES simulations for inhalation flow rates of: (a) $30 \mathrm{~L} / \mathrm{min}$, (b) $60 \mathrm{~L} / \mathrm{min}$, and (c) $90 \mathrm{~L} / \mathrm{min}$ 


\subsubsection{Comparison of measured and computed regional particle deposition fraction}

Figure 7.15 shows a comparison of measured particle deposition and computed deposition from LES simulations within each region for a range of particle diameters and flow rates. The experiments and simulations agree quite well, although the LES results show a slight under-prediction for larger particles in the anterior region that is most pronounced at higher flow rates. As discussed above, this is most likely attributed to differences in actual and simulated wall roughness as well as any potential influence of subgrid scale turbulence on particle dispersion and deposition.

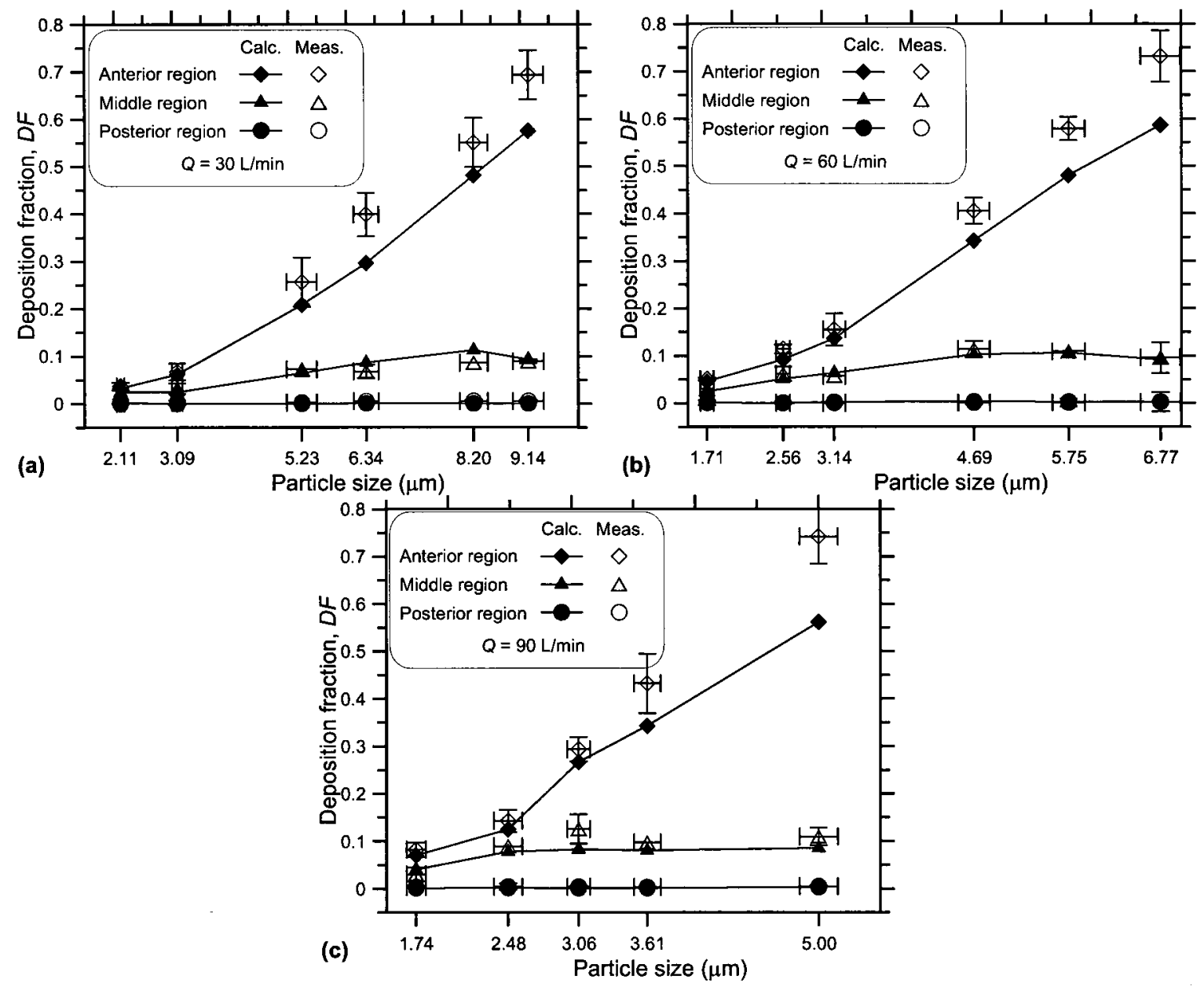

Figure 7.15 Measured and LES results for regional deposition for various sizes of particles at inhalation flow rates of: (a) $30 \mathrm{~L} / \mathrm{min}$, (b) $60 \mathrm{~L} / \mathrm{min}$, and (c) $90 \mathrm{~L} / \mathrm{min}$ 


\subsubsection{Fitting curves for regional particle deposition fraction}

For any given particle diameter and flow rate, more deposition occurs in the anterior region than in either the middle or posterior regions; however, the ratio of deposition among regions varies with particle diameter. These results can be correlated with the impaction parameter as shown in Figure 7.16.

The curves on Figure 7.16 are empirical, second order polynomial fits $(D F=a+b \cdot I P$ $\left.+c \cdot I P^{2}\right)$, and the values of the constants $a, b$, and $c$ are included in Table 7.4. Deposition in the posterior region is essentially negligible because the posterior part contains less than $10 \%$ of the total length of the nasal cavity.

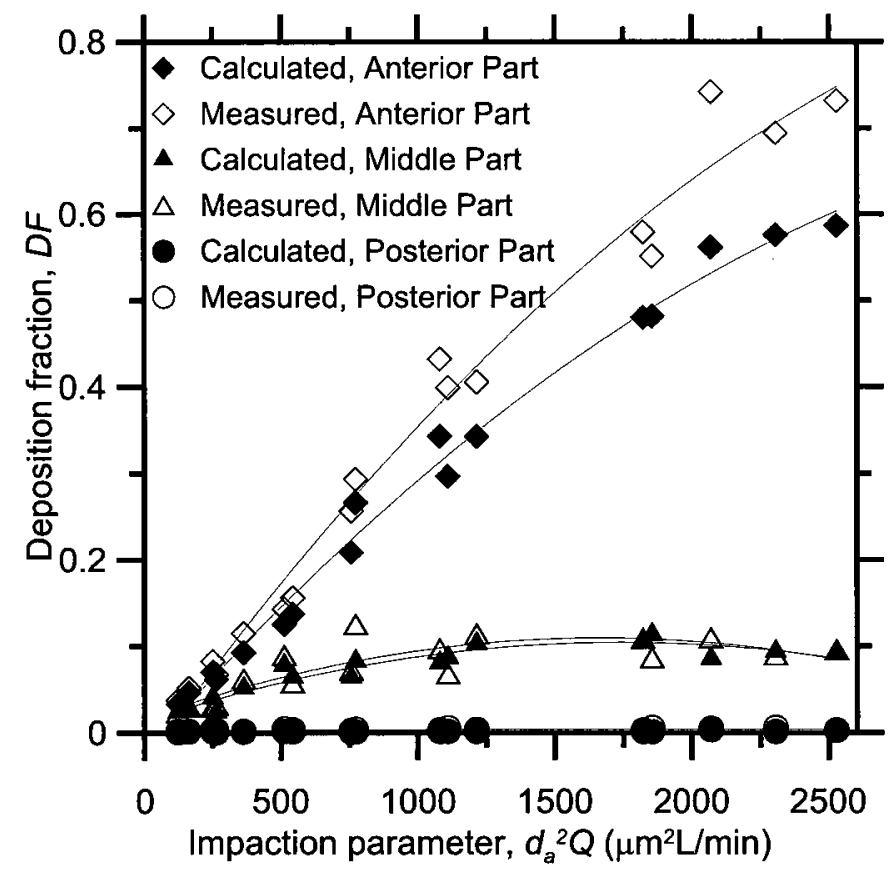

Figure 7.16 Particle deposition in the three regions of the nasal cavity correlated with the impaction parameter, $d_{a}^{2} Q$

The trends of deposition as a function of the impaction parameter reveal how the relative amounts of particles deposited in each region can vary under different conditions. 
Peak deposition in the middle region occurs at an impaction parameter of approximately $1500 \mu \mathrm{m}^{2} \mathrm{~L} / \mathrm{min}$; however, only $10 \%$ of the total particles are deposited at this condition, which equates to $\sim 17.5 \%$ of the total deposited particles. At an impaction parameter of $200 \mu \mathrm{m}^{2} \mathrm{~L} / \mathrm{min}$, even fewer (only $\sim 3.7 \%$ ) of the total particles entering the nasal cavity are deposited in the middle region, but this amount represents a much larger fraction $(\sim 42 \%)$ of the total particles that are deposited. In any case, regional deposition results suggest that significant quantities of particles can be deposited in the anterior part of the nasal cavity, thus it is important to consider the geometry of the nasal inlet and the nasal valve when modeling the nasal cavity.

Table 7.4 Curve fit parameters for regional deposition data

\begin{tabular}{|c|c|c|c|c|}
\hline Curve & $\mathbf{a}$ & $\begin{array}{c}\mathbf{b} \\
\left(\mathrm{min} / \mu \mathrm{m}^{2} \mathrm{~L}\right)\end{array}$ & $\begin{array}{c}\mathbf{c} \\
\left(\mathrm{min}^{2} / \mu \mathrm{m}^{4} \mathrm{~L}^{2}\right)\end{array}$ & $\mathbf{r}^{2}$ \\
\hline Anterior region, experimental data & -0.035 & $4.404 \times 10^{-4}$ & $-5.179 \times 10^{-8}$ & 0.982 \\
\hline Anterior region, LES data & -0.024 & $3.594 \times 10^{-4}$ & $-4.411 \times 10^{-8}$ & 0.991 \\
\hline Middle region, experimental data & 0.016 & $1.112 \times 10^{-4}$ & $-3.375 \times 10^{-8}$ & 0.718 \\
\hline Middle region, LES data & 0.013 & $1.048 \times 10^{-4}$ & $-3.010 \times 10^{-8}$ & 0.901 \\
\hline
\end{tabular}

\subsubsection{Effect of size of nasal cavity on particle deposition fraction}

Simulation on total particle deposition fraction was also performed using RANS/EIM approach on two nasal cavities that were shrunk for purposes of comparison. The standardized nasal cavity model was scaled using factors of 0.8 and 0.4 , which provided $20 \%$-shrunk and $80 \%$-shrunk nasal cavity models, respectively. The simulation settings for the shrunk nasal cavity model are the same as those described in Chapter 6 . Figure 7.17 shows the simulation results on the two shrunk nasal cavity models, and the results 
using standardized nasal model and in vivo data summarized by Cheng (2003) are also presented. It can be seen that the deposition fraction for the shrunk models are higher than the values of the standardized model for the same impaction parameter. The results of $20 \%$-shrunk model are close to the lower confidence level interval line, and results of $40 \%$-shrunk model are close to the averaged in vivo data (Cheng, 2003). It is conjectured here that the difference in deposition is due to nasal airway geometry. Smaller nasal cavity geometry (smaller minimum cross-sectional area) results in higher particle deposition, which is consistent with the conclusion drawn by Swift (1981), in which deposition fraction for the infant casts was higher than in the adult casts for the same impaction parameter.

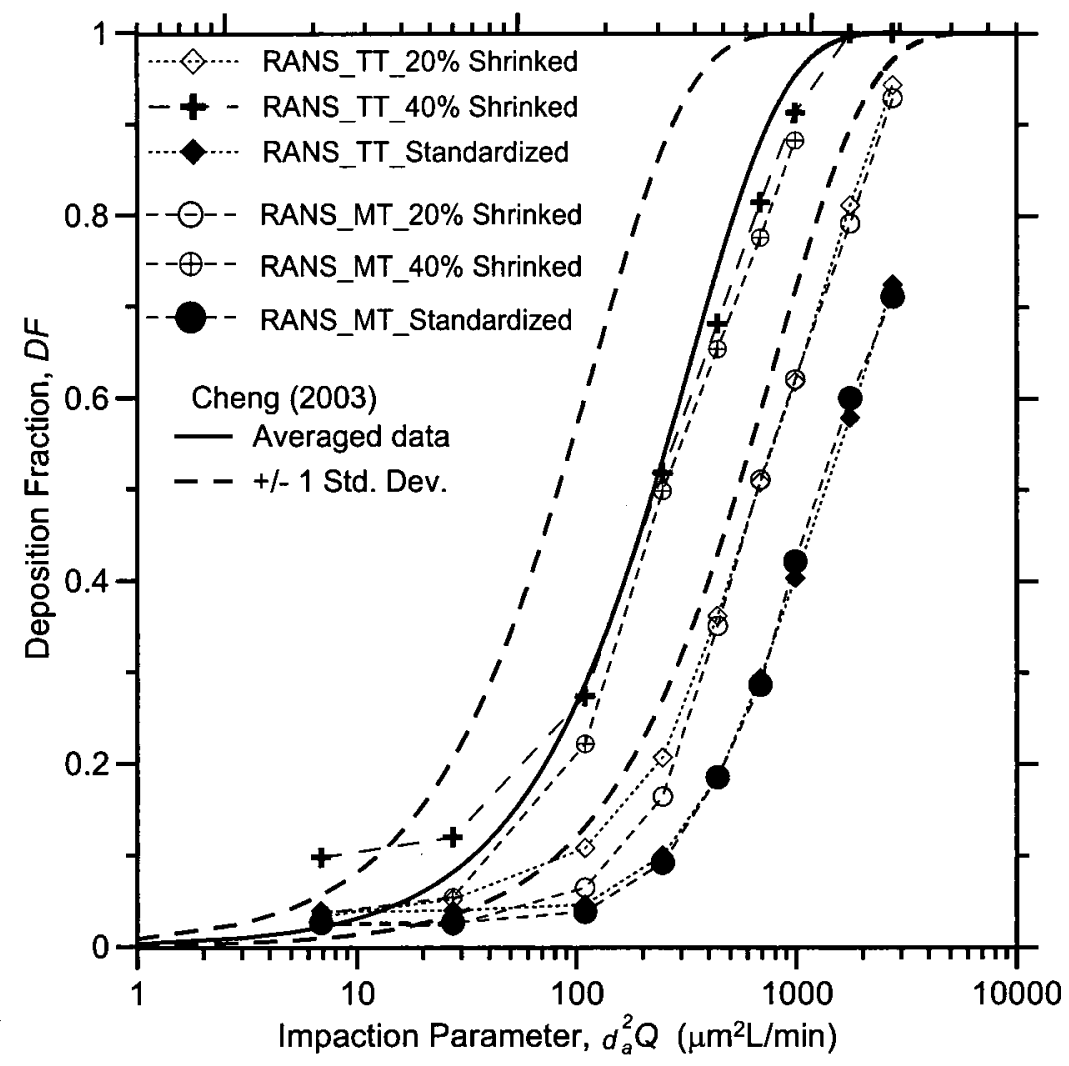

Figure 7.17 Deposition results using shrunk nasal cavity models 


\section{Chapter 8: Conclusions and Future work}

\subsection{Conclusions}

A novel, standardized nasal cavity model has been created by carefully aligning and processing 30 sets of $\mathrm{CT}$ scans of human nasal cavities. Through digital image processing, the left and right nasal cavities were separately analyzed to produce a standardized nasal cavity model that closely matched the median cross-sectional area characteristic of the 60 individual geometries, while retaining the unique geometric features of the human nasal cavity. Using this "Carleton-Civic" standardized geometry of the human nasal cavity, airflow patterns were simulated, and aerosol deposition was studied both numerically and experimentally.

Deposition data from in vitro experiments in the Carleton-Civic standardized human nasal cavity geometry fell within available published in vitro data from individual subjects. Data also correlated well with cited in vivo data, although deposition was lower for identical values of the impaction parameter. This difference is attributable to a combination of the absence of nasal hairs in the in vitro model, differences in wall roughness, and unquantified uncertainties in the cited in vivo measurements.

Simulated results of airflow show that independent of the turbulence level at the inlet, non-negligible turbulence kinetic energy will be present in the first half of the computational domain, indicating that turbulence models are necessary for the range of the inhalation flow rates $(15-90 \mathrm{~L} / \mathrm{min})$ simulated in this study. For each flow rate, the profile of mean turbulence kinetic energy shows similar trends, and the peak value of 
mean turbulence kinetic energy appears at a dimensionless distance from the nostril equal to 0.35 , which coincides with the beginning and the frontal location of the turbinates. Further into the cavity, the turbulent energy quickly dissipates, indicating that the downstream flow is essentially laminar.

Results of deposition experiments were generally well-predicted by the numerical simulations, with some exceptions for large values of the impaction parameter. Regional deposition data derived from the standardized model revealed that, for the range of conditions studied, the majority of deposition occurs in the anterior region of the nasal cavity and specifically in the region of the nasal valve, and that the relative amounts of deposition within the nasal cavity vary significantly with impaction parameter. Both measurements and LES results of regional deposition efficiencies suggest that the geometry of the nasal inlet through to the nasal valve is critical in correctly modeling deposition in the nasal passageway. Considering the important role of the turbinates in the absorption of drug delivered to the nasal cavity, the trends of the regional particle fractions have significance for the determination of preferred particle sizes and could have application in pharmaceutical research.

The Carleton-Civic nasal geometry produced in this work will be useful to researchers and physicians in studying deposition of inhaled aerosols, and also will serve as a candidate standard for aerosol deposition testing in the pharmaceutical industry. The use of a standardized geometry could aid in development of more accurate experimental and numerical predictions of pharmaceutical aerosol deposition and raises possibilities for new insight, faster development, and reduced costs of better drug delivery devices. As a diagnostic tool, the CT scan of a patient could be processed and compared against 
this standardized model to indicate deviation from the present average characteristic values, such as cross-sectional area, nostril area, or generic shape. The specific procedure developed in this study could also be applied in the future in comprehensive studies involving different identifiable groups in the population, as more CT scan or MRI data become available.

\subsection{Future work}

Despite the success of this project, several important research questions remain in the quest to fully understand and predict aerosol deposition in the human nasal cavity. Some of these future research areas are as follows:

1. Airflow patterns have been investigated numerically using the novel standardized human nasal cavity model. Fluid flow measurements using PIV (Particle Image Velocimetry) would be helpful to validate present simulation results of airflow patterns.

2. In present work, the inertial parameter was changed by mostly changing the flow rate instead of having larger particles. Further investigation on particle deposition for lower inhalation flow rates and larger particles would be worth performing.

3. Present measured particle deposition fractions were obtained using a physical replica, which was divided into three parts. While this is an improvement over most previous studies which have only measured total deposition, the present investigation of regional deposition results was still limited. 
Based on results of this work, a physical nasal model could be manufactured and assembled to obtain more details of the regional particle deposition fraction in regions of specific interest, i.e., within the existing anterior section.

4. In the present simulations, the inner wall of the nasal cavity model was simplified as a hydraulically smooth wall without nasal hairs. From the discussion in Chapter 5 and Chapter 7, it can be seen that the effect of nasal hairs and wall roughness on airflow patterns and particle depositions patterns are non-negligible. Improved simulation methodology could take these factors into account and to provide more realistic results of particle deposition and airflow patterns. Also, experimentally investigating the influence of nasal hairs and wall roughness would be very helpful to validate simulation results.

5. In present simulation and experimental work, nasopharynx was not included in the standardized nasal cavity model. An improved nasal model could be generated including the nasopharynx and could be used to study its influence on the airflow patterns and deposition patterns in the nasal cavity.

6. As discussed in section 2.3.2.2, Häußermann et al. (2002) pointed out that human cyclic breathing patterns had significant influences on the particle deposition for flow rates higher than $10 \mathrm{~L} / \mathrm{min}$. The influence of realistic human breathing patterns on particle deposition could be investigated in the future using the standardized human nasal cavity model.

7. Building on the methodology used to create the Civic-Carleton standardized human nasal cavity, an intensive study could be performed to investigate the influence of inter-subject variability of nasal anatomic geometry 
on particle deposition. Ideally, the relationship between nasal anatomic characteristic dimensions for subgroups of population (such as gender, age, ect.) and particle deposition patterns could be found and that could be applied for the prediction of particle deposition in human nasal cavity. 


\section{References}

Adamson JE. Constriction of the internal nasal valve in rhinoplasty: Treatment and prevention. Annals of Plastic Surgery 18: C114-C121, 1987.

AIAA, "Guide for the Verification and Validation of Computational Fluid Dynamics Simulations," AIAA G-077-1998, 1998.

Akselvoll K, and Moin P. Application of the dynamic localization model to large-eddy simulation of turbulent flow over a backward facing step. Engineering Applications of Large Eddy Simulation (Edited by S. Ragab and U. Piomelli), ASME FED 162: C1-C6, 1993.

American Society of Mechanical Engineers. Measurement Uncertainty, Instruments and Apparatus. ANSI/ASME PTC 19.1, 1985.

Barry PW, and O'Callaghan C. Nebuliser therapy in childhood. Thorax 52: C78-C88, 1997.

Basset AB. A treatise on hydrodynamics, $2^{\text {nd }}$ edition. Cambridge: Deighton, Bell; (1961) New York: Dover.

Bauman RP, and Poe R. Absorbtion spectroscopy. New York: Wiley, 1962.

Bennett WD, and Zeman KL. Effect of race on fine particle deposition for oral and nasal breathing. Inhalation Toxicology 17: C641-C648, 2005.

Berlemont A, Desjonqueres P, and Gousbet G.. Particle Lagrangian simulation in turbulent flows. International Journal of Multiphase Flow 16: C19-C34, 1990.

Bilgen E, Arbour P, and Turik PT. On the resistance of air flow through the nose. New York: ASME Publication, United Engineering Center, 1974.

Burry D, and Bergeles G. Dispersion of particles in anisotropic turbulent flows. International Journal of Multiphase Flow 19: C651-C664, 1993.

Breuer M, Baytekin HT, and Matida EA.Prediction of aerosol deposition in bends using LES and an efficient tracking method. Journal of Aerosol Science 37(11): C1407C1428, 2006.

Brooke JW, Hanratty TJ, and Mclaughlin JB. Free-flight mixing and deposition of aerosols. Physics of Fluids 6, C3404-C3415, 1994.

Buenting JE, Dalston RM, Smith TL, and Smith AF. Artifacts associated with acoustic rhinometric assessment of infants and young children: a model study. Journal of Applied Physiology 77: 2558-2563, 1994.

Canny J. A Computational Approach to Edge Detection. IEEE Trans. Pattern Analysis and Machine Intelligence 8: C679-C714, 1986.

Chan TL, Schreck RM, and Lippmann M. Effect of the laryngeal jet on particle deposition in the human trachea and upper bronchial airways. Journal of Aerosol Science 11(5-6): C447-C459, 1980.

Chen XQ, and Pereira JF. Computation of particle dispersion in turbulent liquid flows 
using an efficient Lagrangian Trajectory. International Journal for Numerical Methods in Fluids 26: C345-C364, 1998.

Cheng KH, Cheng YS, Yeh HC, Guilmette RA, Simpson SQ, and Yang YH. In vivo measurements of nasal airway dimensions and ultrafine aerosol deposition in the human nasal and oral airways. Journal of Aerosol Science 27(5): C785-C801, 1996.

Cheng YS, Yamada Y, Yeh HC, and Swift DL. Diffusional deposition of ultrafine aerosols in a human nasal cast. Journal of Aerosol Science 19: C741-C751, 1988.

Cheng YS, Yeh HC, Guilmette RA, Simpson SQ, Cheng KH, and Swift DL. Nasal deposition of ultrafine particles in human volunteers and its relationship to airway geometry. Aerosol Science and Technology 25: C274-C291, 1996.

Cheng YS, Holmes TD, Gao J, Guilmette RA, Li S, Surakitbanharn Y, and Rowlings C. Characterization of nasal spray pumps and deposition pattern in a replica of the human nasal airway. Journal of Aerosol Medicine 14: C267-C270, 2001.

Cheng YS. Aerosol deposition in the extra-thoracic region. Aerosol Science and Technology 37: C659-C671, 2003.

Churchill SE, Shackelford LL, Georgi JN, and Black MT. Morphological variation and airflow dynamics in the human nose. American Journal of Human Biology 16(6): C625-C638, 2004.

Cohen BS, and Asgharian B. Deposition of ultrafine particles in the upper airways: An empirical analysis. Journal of Aerosol Science 21(6): C789-C797, 1990.

Coleman HW, and Steele WG. Experimentation and uncertainty analysis for engineers, $2^{\text {nd }}$ edition. New York, NY: John Wiley \& Sons, C6-C15, 1999.

Corey JP, Gunfor A, Nelson R, Fredberg J, and Lai V. A comparison of the nasal cross-sectional areas and volumes obtained with acoustic rhinometry and magnetic resonance imaging. Otolaryngology-Head and Neck Surgery 117(4): C349-C354, 1997.

Corrsin S, and Lumley $\mathbf{J}$. On the equation for a particle in turbulent fluid. Applied Science Reasearch Section A 6: C114-C116, 1956.

Crowe CT. Multiphase Flow Handbook. CRC Press, 2005.

Damadian R, Goldsmith M, and Minkoff $L$. NMR in cancer: XVI. FONAR image of the live human body. Physiological Chemistry and Physics 9: C97-C100, 1977.

Damm M, Vent J, Schmidt M, Theissen P, Eckel HE, Lotsch J, and Hummel T. Intranasal volume and olfactory function. Chemical Sciences 27(9): C831-C839, 2002.

Davis SS, and Eccles R. Nasal congestion: Mechanisms, measurement and medications. Core information for the clinician. Clinical Otolaryngology 29: C659-C666, 2004.

De Boor C. A Practical Guide to Splines. Springer-Verlag, C113-C114, 1978.

DeHaan, WH, and Finlay WH. In vitro monodisperse aerosol deposition in a mouth and throat with six different inhalation devices. Journal of Aerosol Medicine, 14(3): C361C367, 2001.

Doorly D, Taylor DJ, Franke P, and Schroter RC. Experimental investigation of nasal 
airflow. Proceedings of the Institution of Mechanical Engineers, Part H: Journal of Engineering in Medicine 222(4): C439-C453, 2008.

Dwibhashyam VSNM, and Nagappa AN. Strategies for enhanced drug delivery to the central nervous system. Indian Journal of Pharmaceutical Science March - April: C145C153, 2008.

Elgobashi S. On predicting particle-laden turbulent flows. Applied Scientific Research 52: C309-C329, 1994.

Etherington G, Smith JRH, Bailey MR, Dorrian MD, Shutt AL, and Youngman MJ. Deposition and clearance of inhaled particles in the human nasal passage: Implications for dose assessment. Radiation Protection Dosimetry 79: C249-C252, 1998.

Fidone SJ, and Gonzalez C. Initiazation and control of chemoreceptor activity in the carotid body. In: Handbook of Physiology. The Respiratory System. Control of Breathing. Bethesda. The American Physiological Society 3(II), C247-C312, 1986.

Finlay WH. The Mechanics of Inhaled Pharmaceutical Aerosols: An Introduction. Academic Press, New York, 2001.

Fishman AP, and Geiger SR. Handbook of physiology: the respiratory system. Bethesda, MD: American Physiological Society. 247-261, 1985.

Fouke JM, and Strohl KP. Effect of position and lung volume on upper airway geometry. Journal of Applied Physiology 63: 375-380, 1987.

Freitas CJ. The issue of numerical uncertainty. Applied Mathematical Modeling 26: C237-C248, 2002.

Frey WH. Intranasal delivery: Bypassing the blood-brain barrier to deliver therapeutic agents to the brain and spinal cord. Drug Delivery Technology 2(5): C46-C49, 2002.

Friedlander SK, and Johnstone HF. Deposition of suspended particles from turbulent gas stream. Industrial and Engineering Chemistry Research Fundamentals 49(7): C1151C1156, 1951.

Friedlander SK, Johnstone HF. Deposition of suspended particles from turbulent gas streams. Industrial and Engineering Chemistry 49: C1151-C1156, 1957.

Ganic EN, and Mastanaiah KM. Investigation of droplet deposition from a turbulent gas stram. International Journal of Multiphase Flow 7: C401-C422, 1981.

Garcia GJM, Tewksbury E., Wong BA, and Kimbell JS. Interindividual variability in nasal filtration as a function of nasal cavity geometry. Journal of Aerosol Medicine and Pulmonary Drug Delivery 22: C139-C155, 2009.

Gehr P, and Heyder J. Particle-Lung Interactions. Vol 143 in Lung Boilogy in Health and Diease (Executive editor: Lenfant C), Marcel Dekker Inc., New York, 2000.

Giacomelli-Maltoni G, Melandri C, Prodis V, and Tarroni, G. Deposition efficiency of monodisperse particles in human respiratory tract. American Industrial Hygiene Association Journal: C603-C610, 1972.

Girardin M, Bilgen E, and Arbour P. Experimental study ofvelocity fields in a human nasal fossa by laser anemometry. Annals of Otology, Rhinology and Laryngology 92: 
C231-C236, 1983.

Giroux M, Hwang P, and Prasad A. Controlled particle dispersion: Applying vertical flow to optimize nasal drug deposition. Drug Delivery technology 5(3): C44-C49, 2005.

Gosman AD, and Ioannides E. Aspects of Computer Simulation of Liquid-fueled Combustors. In: AIAA 19th Aerospace Science Meeting, 81, 1981.

Govan AH, Hewitt G F, and Ngan CF. Particle motion in a turbulent pipe flow. International Journal of Multiphase Flow 15: C471-C481, 1989.

Gradon L, and Podgorski A. Experimental study on fibrous particle deposition in the human nasal cast. Journal of Aerosol Science 23: C469-C472, 1992.

Graham DI, and James PW. Turbulent dispersion of particles using eddy interaction models. International Journal of Multiphase Flow 22(1): C157-C175, 1996.

Grothberg JB. Respiratory fluid mechanics and transport progress. Annual Review of Biomedical Engineering 3: C421-C457, 2001.

Guha A. Transport and deposition of particle $s$ in turbulent and laminar flow. Annual Review of Fluid Mechanics 40: C311-C341, 2008

Guilmette RA, and Gagliano, TJ. Construction of a Model of Human Nasal Airways Using In Vivo Orphological Data. Annals of Occupational Hygiene 38: C69-C75, 1994.

Guilmette RA, Cheng YS, and Griffith WC. Characterising the variability in adult human nasal airway dimensions. Annals of Occupational Hygiene 41: C491-C496, 1997.

Gurman JL, Lioy PJ, Lippmann M, and Schlesinger RB. Particle deposition in replicate casts of the human upper tracheo-bronchial tree under constant and cyclic inspiratory flow. 2. Empirical-model. Aerosol Science and Technology 3(3): C253-C257, 1984.

Hahn I, Scherer PW, and Mozell MM. Velocity profiles measured for airflow through a large-scale model of the human nasal cavity. Journal of Applied Physiology 75: C2273C2287, 1993.

Häußermann S, Bailey AG, Bailey MR, Etherington G, and Youngman M. The influence of breathing patterns on particle deposition in a nasal replicate cast. Aerosol Science 33: C923-C933, 2002.

Heenan AF, Matida EA, Pollard A, and Finlay WH. Experimental measurements and computational modeling of the flow in an idealized extrathoracic airway. Experimental Fluids 35: C70-C84, 2003.

Heyder J, and Rudolf G. Deposition of Aerosol in the Human Nose. In: Inhaled Particle IV, Pergamon Press, Oxford, C107-C125, 1977.

Heyder J, Gebhart J, Rudolf G, Schiller CF, and Stahlhofen W. Deposition of particles in the human respiratory tract in the size ranges $0.005-15 \mu \mathrm{m}$. Journal of Aerosol Science 17: C811-C825, 1986.

Hilberg O, Jackson AC, Swift DL, and Pedersen OF. Acoustic rhinometry: evaluation of nasal cavity geometry by acoustic reflection. Journal of Applied Physiology 66(1): C295-C303, 1989. 
Hinds WC. Aerosol Technology. Properties, Behavior, and Measurement of Airborne Particles. John Wiley and Sons, New York, U.S.A., 1982.

Hennick EA, and Lightstone MF. A Comparison of Stochastic Separated Flow Models for Particle Dispersion in Turbulent Flows. Energy and Fuels, 14: C95-C103, 2000.

Hinshaw DS, Bottomley PA, and Holland GN. Radiographic thin-section image of the human wrist by nuclear magnetic resonance. Nature 270: C722-C723, 1973

Hinze JO. Turbulence. McGraw-Hill, New York, U.S.A., 1975.

Hounam RF, Black A, and Walsh M. Deposition of aerosol particles in the nasopharyngeal region of the human respiratory tract. Nature 221: C1254-C1255, 1969.

Hooper, RG. Forced inspiratory nasal flow-volume curves: A simple test of nasal airflow. Mayo Clinic Proceedings 76:C990-C994, 2001.

Hopkins LM, Kelly JT, Prasad AK, and Wexler AS. Particle image velocimetry measurements in complex geometries. Experimental Fluids 29: C91-C95, 2000.

Hussain AA. Intranasal drug delivery. Advanced Drug Delivery Reviews 29: C39-C49, 1998.

Hutchinson P, Hewitt GF, and Dukler AE. Deposition of liquid or solid dispersions from turbulent gas streams: a stochastic model. Chemical Engineering Science 26: C419C439, 1971.

Illum L. Transport of drugs from the nasal cavity to the central nervous system. European Journal of Pharmaceutical Science 11:C1-C18, 2000.

Inthavong K, Tian ZF, Li HF, Tu JY, Yang W, and Xue CL. A numerical study of spray particle deposition in a human nasal cavity. Aerosol Science Technology 40: C1034C1033, 2006.

Itoh H, Smaldone GC, Swift DL, and Wagner HN. Mechanicms of aerosol deposition in a nasal model. Journal of Aerosol Science 16: C529-C534, 1985.

Jackson AC, Butler JP, Millet EJ, Hoppin FG, and Dawson SV. Airway geometry by analysis of acoustic pulse response measurements. Journal of Applied Physiology 43: C523-C536, 1977.

Jackson R. Locally averaged equations of motion for amixture of identical spherical particles and a Newtonian fluid. Chemical Engineering Science 52(15): C2457-C69, 1997.

Janssens HM, DeJongste JC, Fokkens WJ, Robben SGF, Wouters K, and Tiddens H. The Sophia anatomical infant nose-throat (SAINT) model: A valuable tool to study aerosol deposition in infants. Journal of Aerosol Medicine 14: C433-C441, 2001.

James PW, Hewitt GF, and Whalley PB. Droplet motion in two-phase flow. In: Proc. ANS/ASME/NRC International Topical Meeting on Nuclear Reactor Thermal-Hydraulics, C1484-C1503, 1980.

Johnston JR, and Schroter RC. Deposition of particles in model airways. Journal of Applied Physiology 47: C947-C953, 1979.

Kallio G.A, and Reeks MW. A numerical simulation of particle deposition in turbulent 
boundary layers. International Journal of Multiphase Flow 15: C433-C446, 1989.

Kelly JT, Prasad AK, and Wexler AS. Detailed flow patterns in the nasal cavity, Journal of Applied Physiology 89: C323-C337, 2000.

Kelly JT, Asgharian B, Kimbell JS, and Wong BA. Particle deposition in human nasal airway replicas manufactured by different methods. Part I: Inertial regime particles. Aerosol Science Technology 38: C1063-C1071, 2004.

Kelly JT, Asgharian B, Kimbell JS, and Wong BA: Particle deposition in human nasal airway replicas manufactured by different methods. Part II: Ultrafine particles. Aerosol Science Technology 38: C1072-C1079, 2004.

Kesavanathan J, and Swift DL. Human nasal passage particle deposition: The effect of particle size, flow rate and anatomical factors. Aerosol Science and Technology 28(5): C457-C463, 1998.

Kim CS, and Iglesias AJ. Deposition of inhaled particles in bifurcating airway models-I. Inspiratory deposition. Journal of Aerosol Medication 2: C1-C14, 1989.

Kim I, Elgobashi S, and Sirignano WA. ON the equation for spherical particle motion: Effect of Reynolds and acceleration numbers. Journal of Fluid Mechanics 367: C221C253, 1998.

Kim SK and Chung SK. An investigation on airflow in disordered nasal cavity and its corrected models by tomographic PIV. Measurement Science and Technology 15: C1090C1096, 2004.

Kimbell JS, Subramaniam RP, Gross EA, Schlosser PM, and Morgan KT. Dosimetry modeling of inhaled formaldehyde: Comparisons of local flux predictions in the rat, monkey, and human nasal passages. Toxicological Sciences 64:C100-C110, 2001.

Kimbell JS. Nasal Dosimetry of Inhaled Gases and Particles: Where Do Inhaled Agents Go in the Nose? Toxicological Pathology 34(3): C270-C273, 2006.

Kline SJ. Similitude and Approximation Theory. Springer-Verlag, New York, 1986.

Knuth DE. The Art of Computer Programming, Volume 2: Seminumerical Algorithms. Second edition. Reading, Massachusetts: Addison-Wesley, 1981.

Kramer PR, and Wray S. Novel gene expressed in nasal region influences outgrowth of olfactory axons and migration of luteinizing hormone releasing hormone (LHRH) neurons. Genes \& Development 14: C1824-C1834, 2000.

Landahl HD, and Black S. Penetration of air-borne particulates through the human nose. Journal of Industrial Hygiene and Toxicology 29: C269-C277, 1947.

Lapple CE, and Shepherd CB. Calculation of particle trajectories. Industrial and Engineering Chemistry 32: C605-C617, 1940.

Lauterbur PC. Image Formation by Induced Local Interactions: Examples of Employing Nuclear Magnetic Resonance. Nature 242: 190-191, 1973.

Lenders H, and Pirsig W. Diagnostic value of acoustic rhinometry: Patients with allergic and vasomotor rhinitis compared with normal controls. Rhinology 28: C5-C16, 
1990.

Lennon S, Zhang Z, Lessmann R, and Webster S. Experiments on particle deposition in the human upper respiratory system. Aerosol Science and Technology 28: C464-C474, 1989.

Lilly DK. On the application on the eddy viscosity concept in the inertial sub-range of turbulence. NCAR Manuscript, C195-C200, 1996.

Liu BYH, and Pui DYH. Electrical neutralization of aerosols. Journal of Aerosol Science 5: C461-C472, 1974.

Liu Y, Matida EA, Gu J, and Johnson MR. Numerical simulation of aerosol deposition in a 3-D human nasal cavity using RANS, RANS/EIM, and LES. Journal of Aerosol Science 38(7): C683-C700, 2007.

Liu Y, Johnson MR, Matida EA, Kherani S, and Marsan J. Creation of a standardized geometry of the human nasal cavity, Journal of Applied Physiology 106: C784-C795, 2009.

Luo XY, Hinton JS, Liew TT, and Tan KK. LES modelling of flow in a simple airway model. Medical Engineering and Physics 26: C403-C413, 2004.

MacInnes JM, and Bracco FV. Stochastic particle dispersion modeling and the tracerparticle limit. Physics of Fluids A4(12): C2809-C2824, 1992.

Mashayek F, and Pandya, RVR. Analytical description of particle/droplet-laden turbulent flows. Progress in Energy and Combustion Science 29(4): C329-C378, 2003.

Mathison S, Nagilla R, and Kompella UB. Nasal route for direct delivery of solutes to the central nervous system: Fact or fiction? Journal of Drug Targeting 5: C413-C441, 1998.

Matida EA, DeHaan WH, Finlay WH, and Lange CF. Simulation of particle deposition in an idealized mouth with different small diameter inlets. Aerosol Science and Technology 37: C924-C932, 2003.

Matida EA, Finlay WH, Lange CF, and Grgic B. Improved numerical simulation of aerosol deposition in an idealized mouth-throat. Journal of Aerosol Science 35: C1-C19, 2004.

Marieb EN. Human anatomy and physiology. Menco Park, CA: Benjamin/Cummings, C744-C747, 1995.

Maxey MR, and Riley JJ. Equation of motion for a small rigid sphere in a nonuniform flow. Physics of Fluids A4(12): C2809-C2824, 1983.

McCoy DD, and Hanratty TJ. Rate of deposition of droplets in annular two-phase flow. International Journal of Multiphase Flow 3: C319-C31, 1977.

Mclaughlin, JB. Aerosol particle deposition in numerically simulated channel flow. Physics of Fluids A1: C1211-C1244, 1989.

Mei R. Velocity fidelity of flow tracer particles. Experimental Fluids 22: C1-C13, 1996.

Min YG, and Jang YJ. Measurements of cross-sectional area of the nasal cavity by 
acoustic rhinometry and CT scanning. Laryngoscope 105: C757-C759, 1995.

Minn A, Leclerc S, Heydel JM, Minn AL, Denizot C, and Cattarelli M Drug transport into the mammalian brain: The nasal pathway and its specific metabolic barrier. Journal of Drug Targeting 10(4): C285-C296, 2002.

Murry DP, EL-Hakim H, Ahsan F, and Nunez DA. Does synchronous nasal surgery increase the risk of postoperative haemorrhage in adult patients undergoing tonsillectomy? The Journal of Laryngology \& Otology 117:C707-C709, 2003.

Narayan KM, Boyle JP, Thompson TJ, Sorensen SW, and Williamson DF. Lifetime risk for diabetes mellitus in the United States. Journal of the American Medical Association 290(14):C1884-C1890, 2003.

Ossen CW. Hydrodynamik. Leipzig: Akademische Verlag, 1927.

Overton JH, Kimbell JS and Miller FJ. Dosimetry modeling of inhaled formaldehyde: the human respiratory tract. Toxicological Sciences 64(1): C122-C134, 2001.

Pandya RVR, and Mashayek F. Two-Fluid Large-eddy Simulation Approach for Particle-Laden Turbulent Flows. International Journal for Heat and Mass Transfer 45(24): C4753-C4759, 2002.

Pang KP. One-stage nasal and multi-level pharyngeal surgery for obstructive sleep apnoea: safety and efficacy. The Journal of Laryngology \& Otology 119:C272-C276, 2005.

Park KI, Brucker C, and Limberg W. Experimental study of velocity fields in a model of human nasal cavity by DPIV. In: Laser Anemometry Advances and Applications: Proceedings of the 7th International Conference, edited by Ruck B, Leder A, and Dopheide D. Karlsruhe, Germany: University of Karlsruhe, 1997.

Patni P, Varghese D, Balekara N, and Jain DK. Needle-free insulin drug delivery. Indian Journal of Pharmacy Science 68(1):C7-C12, 2006.

Pattle, RE. The retention of gases and particles in the human nose, In: C.N.Davies, (ed.), Inhaled Particles and Vapours, Pergamon Press, Oxford,UK, C302-C309, 1961.

Press WH, Flannery BP, Teukolsky SA, and Vetterling WT. Numerical Recipes in Pascal: The Art of Scientific Computing. New York: Cambridge University Press, 1989.

Raj P, Stableforth DE, and Morgan DW. A prospective study of nasal disease in adult cystic fibrosis. The Journal of Laryngology \& Otology 114:C260-C263, 2000.

Rajagopal MR, and Jerry P. Applied anatomy and physiology of the airway and breathing. Indian J. Anaesth 49(4): C251-C256, 2005.

Reber M, Rahm F, and Monnier P. The role of acoustic rhinometry in the pre- and postoperative evaluation of surgery for nasal obstruction. Rhinology 36(4): C184-C187, 1998.

Rhomberg LR, and Lewandowski TA. Methods for identifying a default cross-species scaling factor. Human and Ecological Risk Assessment 12(6): C1094-1127, 2006.

Roache PJ. Verification and validation in computational science and engineering. Hermosa Publisher: Albuquerque, 1998. 
Robert GH. Forced inspiratory nasal flow-volume curves: A simple test of nasal airflow. Mayo Clinic proceedings 76: C990-C994, 2001.

Roberts ES, Wong VA, McManus BE, Marshall MW, Lancianese S, and Dorman DC. Changes in intracellular pH play a secondary role in Hydrogen sulfide-induced nasal cyttoxicity. Inhalation Toxicology 18(3): C159-C167, 2006.

Roush GC. Epidemiology of cancer of the nose and paranasal sinuses: Current concepts. Head \& Neck Surgery 2: C3-C11, 1979.

Saffman. The lift on small sphere in slow shear flow. Journal of Fluid Mechanics, 22: C385-C400, 1965.

Sarangapani R, and Wexler AS. Modeling aerosol bolus dispersion in human airways. Journal of Aerosol Science30: C1345-C1362, 1999.

Sarkar MA. Drug metabolism in the nasal mucosa. Pharmaceutical Research, 9(1): C1C9, 1992.

Sato Y, and Yamamoto K. Lagrangian measurement of fluid-particle motion in an isotropic turbulent field. Journal of Fluid Mechanics 175: C183-C199, 1987.

Saunders MW, Jones NS, and Kabala JE. Parameters of nasal airway anatomy on magnetic resonance imaging correlate poorly with subjective symptoms of nasal patency. Clinical Otolaryngology \& Applied Sciences 24: C431-C434, 1999.

Schiller VL, and Naumann A. Über die grundlegenden Berechnungen bei der Schwerkraftaufbereitung. Zeitschrift des Vereines Deutscher Ingenieure 77 (12): C318C320, 1933.

Schuen JS, Chen LD, and Faeth GM. Evaluation of a Stochastic Model of Particle Dispersion in a Turbulent Round Jet. AIChE Journal 29(1): C167-C170, 1983.

Schuepp KG, Straub D, Moller A, and Wildhaber JH. Deposition of aerosols in infants and children. Journal of Aerosol Medicine 17: C153-C156, 2004.

Schulz PB, and Heyder J. Particle deposition in the respiratory tract. In: Gehr P and Heyder J, Editors, Particle-Lung interactions. Lung Biology in Health and Disease 143, Dekker: New York, 2000.

Schreck S, Sullivan KJ, Ho CM, and Chang HK. Correlations between flow resistance and geometry in a model of the human nose. Journal of Applied Physiology 75: C1767C1775, 1993.

Schroeter JD, Kimbell JS, Gross EA, Willson GA, Dorman DC, and Tan YM. Application of physiological computational fluid dynamics models to predict interspecies nasal dosimetry of inhaled Acrolein. Inhalation Toxicology 20(3): C227-C243, 2008.

Shanley KT, Zamankhan P, Ahmadi G, Hopke PK, and Cheng,YS. Numerical simulations investigating the regional and overall deposition efficiency of the human nasal cavity. Inhalation Toxicology 20: C1093-C1100, 2008.

Shi H, Kleinstreuer $\mathbf{C}$, and Zhang $\mathbf{Z}$. Modeling of inertial particle transport and deposition in human nasal cavities with wall roughness. Journal of Aerosol Science 38: C398-C419, 2007. 
Smagorinsky JS. General circulation experiments with the primitive equation. Monthly Weather Review 91: C99-C164, 1963.

Squires KD, and Piomelli U. Dynamic modeling of rotating turbulence. In Turbulent Shear flows 9 (Edited by F. Durst, N. Kasagi, B.E. Launder, F.W. Schmidt and J.H. Whitelaw). Springer, Berlin, C71-C83, 1995.

Stahlhofen W, Rudolf G, and James AC. Intercomparison of experimental regional aerosol deposition data. Journal of Aerosol Medication 2: C285-C308, 1989.

Statistics Canada. Canada's Ethnocultural Mosaic, 2006 Census. Catalogue No. 97-562X, April 2008.

Strong JC, and Swift DL. Deposition of ultrafine particles in a human nasal cast. In Proceedings of the first conference of aerosol society Loughborogh,University of Technology, March31-Aprill., C109-C112, 1987.

Subramaniam RP, Asgharian B, Freijer JI, MillerFJ, and SatishAnjilvel S. Analysis of lobar differences in particle deposition in the human lung. Inhalation Toxicology: International Forum for Respiratory Research 15: C1-C21, 2003.

Sullivan M. Rhinoscopy: a diagnostic aid? J of Small Animal Practice 28: C839-C843, 1987.

Suman JD, Laube BL, and Dalby R. Comparison of Nasal Deposition and Clearance of Aerosol Generated by a Nebulizer and Aqueous Spray Pump. Pharmaceutical Research 16:C1648-C1652, 1999.

Suman JD, Laube BL, Lin T, Brouet G, and Dalby R. Relevance of In Vitro Tests of Nasal Solutions to Predict In Vivo Deposition. Pharmaceutical Research 19:C1-C6, 2002.

Suzina AH, Hamzah M and Samsudin AR. Objective assessment of nasal resistance in patients with nasal disease. The Journal of Laryngology \& Otology 114:C260-C263, 2003.

Swift DL, and Proctor DF. Access of air to the respiratory tract. In: Respiratory Defense Mechanisms: Part I, edited by Brian JD, Proctor DF and Reid LM. New York: Dekker, C63-C93, 1977.

Swift DL. Aerosol deposition and clearance in the human upper airways. Annals of Biomedical Engineering 9: C593-C604, 1981.

Swift DL. Inspiratory inertial deposition of aerosols in human airway replicate casts: Implication for the proposed NCRP lung model. Radiation Protection Dosimetry 38: C29-C34, 1991.

Swift DL, and Kesavanathan J. The anterior human nasal passage as a fibrous filter for particles. Chemical Engineering Communications 151: C65-C78, 1996.

Swift DL, and Strong JC. Nasal deposition of ultrafine Po-218 aerosols in human subjects.. Journal of Aerosol Science 27: C1125-C1132, 1996.

Talegaonkar S, and Mishra, PR. Intranasal delivery: An approach to bypass the blood brain barrier. Indian Journal of Pharmacology 36(3): C140-C147, 2004.

Tang H, Tu JY, Li HF, Au-Hijleh B, Xue CC, and Li CG.. Dynamic analysis of airflow features in a 3D real-anatomical geometry of the human nasal cavity. In: 15th 
Australasian Fluid Mechanics Conference The University of Sydney, Sydney,Australia, 2004.

Tchen CM. Mean value and correlation problems connected with the motion of small lparticles in a turbulent field. Ph.D. Dissertation. Delft University, Netherlands, 1947.

Terheyden H, Maune S, Mertens J, and Hilberg $O$. Acoustic rhinometry: validation by three-dimensionally reconstructed computer tomographic scans. Journal of Applied Science 89: C1013-C1021, 2000.

Tian ZF, Inthavong K, and Tu JY. Deposition of inhaled wood dust in the nasal cavity. Inhal Toxicol 19: C1155-C1165, 2007.

Tien C, and Ramarao BV. Granular filtration of aerosols and hydrosols. Elsevier, Oxford, UK, C122-C141, 2007.

Wang LP, and Stock DE. Stochastic trajectory models for turbulent diffusion: Monte Carlo Process Versus Markov Chains. Atmosphere Environment 26A: C1599-C1607, 1992.

Wang Q, and Squires K. Large eddy simulation of particle-laden turbulent channel flow. Physics of Fluids 8: C1207-C1223, 1996.

Wang Y, and James PW. On the Effect of Anisotropy on the Turbulent Dispersion and Deposition of Small Particles. International Journal of Multiphase Flow 25: C551-C558, 1999.

Wang DY, Goh DYT, Ho AKL, Chew FT, Yeoh KH, and Lee BW. The upper and lower airway responses to nasal challenge with house-dust mite Blomia tropicalis. Allergy 58(1): C78-C82, 2003.

Weinhold I, and Mlynski G. Numerical simulation of airflow in the human nose. European Archives of Oto-Rhino-Laryngology 261(8): C452-C455, 2004.

Yu CP. Theories of electrostatic lung deposition of inhaled aerosols. Annals of Occupational Hygiene 29: C219-C227, 1985.

Yu G, Zhang Z, and Lessman R. Fluid flow and particle diffusion in the human upper respiratory system. Aerosol Science and Technology 28: C146-C158, 1998.

Yuu S, Ueno T, and Umekage T. Numerical simulation of the high Reynolds number slit nozzle gas-particle jet using subgrid-scale coupling large eddy simulation. Chemical Engineering Science 56: C4293-C4307, 2001.

Zhang Z, Kleinstreuer C, and Kim CS. Micro-Particle Transport and Deposition in a human Oral Airway Model. Journal of Aerosol Science 33(12): C1635-C1652, 2003.

Zhao K, Scherer PW, Hajiloo SA and Dalton P. Effect of anatomy on human nasal air flow and odorant transport patterns: implications for olfaction. Chemical Senses 29(5): C365-C379, 2004.

Zhao K, Pribitkin EA, Cowart BJ, Rosen D, Scherer PW, and Dalton P. Numerical modeling of nasal obstruction and endoscopic surgical intervention: Outcome to airflow and olfaction. American Journal of Rhinology 20(3): C308-C316, 2006.

Zhou Q, and Leschziner M A. A time correlated stochastic model for particle dispersion 
in anisotropic turbulence. Proceedings of the 8th Turbulent Shear Flows Symposium, Munich, Germany, September, 1991.

Zwartz GJ, and Guilmette RA. A charge coupled device system to image local particle deposition patterns in a model of a human nasal airway. Aerosol Science Technology 30: C489-C504, 1999.

Zwartz GJ, and Guilmette RA. Effect of flow rate on particle deposition in a replica of a human nasal airway. Inhalation Toxicology 13: C109-C127, 2001.

Web reference:

http://www.diabetes.org/about-diabetes.jsp. American Diabetes Association. All About Diabetes.

www.ae.uiuc.edu/loth/CUP/Loth. Loth E. Particles, drops, and bubbles: Fluid dynamics and numerical methods. 


\section{Appendix A: Shear Stress Transport (SST) Turbulence Model}

The standard version of the $k-\omega$ model can be described as follows:

Continuity Equation,

$$
\frac{\partial U_{i}}{\partial x_{i}}=0
$$

Momentum Equation,

$$
\frac{\partial U_{i}}{\partial t}+U_{j} \frac{\partial U_{i}}{\partial x_{j}}=-\frac{1}{\rho} \frac{\partial p}{\partial x_{i}}+\frac{\partial}{\partial x_{j}}\left[\left(v+v_{T}\right)\left(\frac{\partial U_{i}}{\partial x_{j}}+\frac{\partial U_{j}}{\partial x_{i}}\right)\right]
$$

Turbulence Kinetic Energy,

$$
\frac{\partial k}{\partial t}+U_{j} \frac{\partial k}{\partial x_{j}}=P_{k}-\beta_{1} k \omega+\frac{\partial}{\partial x_{j}}\left[\left(v+\sigma_{1} v_{T}\right) \frac{\partial k}{\partial x_{j}}\right]
$$

Specific Dissipation Rate,

$$
\frac{\partial \omega}{\partial t}+U_{j} \frac{\partial \omega}{\partial x_{j}}=\alpha_{1} \frac{\omega}{k} P_{k}-\beta_{1}^{\prime} \omega^{2}+\frac{\partial}{\partial x_{j}}\left[\left(v+\sigma_{1}^{\prime} v_{T}\right) \frac{\partial \omega}{\partial x_{j}}\right]
$$

The $k-\omega$ model assumes that the turbulence viscosity is linked to the turbulence kinetic energy and turbulent frequency via the relation:

$$
\mu_{T}=\rho \frac{k}{\omega}
$$

To get the SST model, the $k-\varepsilon$ model is developed as: 


$$
\begin{gathered}
\frac{\partial k}{\partial t}+U_{j} \frac{\partial k}{\partial x_{j}}=P_{k}-\beta_{2} k \omega+\frac{\partial}{\partial x_{j}}\left[\left(v+\sigma_{2} v_{T}\right) \frac{\partial k}{\partial x_{j}}\right] \\
\frac{\partial \omega}{\partial t}+U_{j} \frac{\partial \omega}{\partial x_{j}}=\alpha_{2} \frac{\omega}{k} P_{k}-\beta_{2}^{\prime} \omega^{2}+\frac{\partial}{\partial x_{j}}\left[\left(v+\sigma_{2}^{\prime} v_{T}\right) \frac{\partial \omega}{\partial x_{j}}\right]+2 \frac{\sigma_{2}^{\prime}}{\omega} \frac{\partial k}{\partial x_{j}} \frac{\partial \omega}{\partial x_{j}}
\end{gathered}
$$

with model constants:

$$
\begin{aligned}
& \beta_{1}=0.09, \quad \alpha_{1}=5 / 9, \quad \beta_{1}^{\prime}=3 / 40, \quad \sigma_{1}=0.5, \quad \sigma_{1}^{\prime}=0.5 \\
& \beta_{2}=0.09, \quad \alpha_{2}=0.44, \quad \beta_{2}^{\prime}=0.0828, \quad \sigma_{2}=1, \quad \sigma_{2}^{\prime}=0.856
\end{aligned}
$$

The production rate of turbulence is given by:

$$
P_{k}=v_{T} 2 S_{i j} \frac{\partial U_{i}}{\partial x_{j}}
$$

The mean-strain-rate tensor is defined by:

$$
S_{i j}=\frac{1}{2}\left(\frac{\partial U_{i}}{\partial x_{j}}+\frac{\partial U_{j}}{\partial x_{i}}\right)
$$

The SST turbulence model is obtained by:

$$
\Phi_{3}=F_{1} \Phi_{1}+\left(1-F_{1}\right) \Phi_{2}
$$

in which, $\Phi_{1}$ is the standard $k-\omega$ model, $\Phi_{2}$ is the developed $k-\varepsilon$ model. The blending functions $F_{1}$ is equal to one near the surface and switches over to zero inside the boundary layer (i.e., a function of the wall distance) and ensure a smooth transition between the two models and are critical to the success of the method. Their formulation is based on the distance to the nearest surface and on the flow variables.

$$
F_{1}=\tanh \left(\arg _{1}^{4}\right)
$$

with 


$$
\arg _{1}=\min \left(\max \left(\frac{\sqrt{k}}{\beta^{\prime} \omega y}, \frac{500 v}{y^{2} \omega}\right), \frac{4 \rho k}{C D_{K W} \sigma_{\omega 2} y^{2}}\right)
$$

where $y$ is the distance to the nearest wall and $v$ is the kinematic viscosity and:

$$
C D_{K \omega}=\max \left(2 \rho \frac{1}{\sigma_{\omega 2} \omega} \frac{\partial k}{\partial x_{i}} \frac{\partial \omega}{\partial x_{i}}, 1.0 \times 10^{-10}\right)
$$

Additionally, Kinematic Eddy Viscosity is defined in order to get correct transport characteristic:

$$
v_{T}=\frac{\alpha_{1} k}{\max \left(\alpha_{1} \omega ; S F_{2}\right)}
$$

in which,

$$
F_{2}=\tanh \left(\arg _{2}^{2}\right)
$$

with

$$
\arg _{2}=\max \left(\frac{2 \sqrt{k}}{\beta^{\prime} \omega y}, \frac{500 v}{y^{2} \omega}\right)
$$

$F_{2}$ is a blending function similar to $F_{1}$, which restricts the limiter to the wall boundary layer, as the underlying assumptions are not correct for free shear flows. 


\section{Appendix B: Large Eddy Simulation}

For an incompressible flow of a Newtonian fluid, the filtered Navier-Stokes Equations solved by the LES are given by the continuity equation

$$
\frac{\partial \bar{u}_{i}}{\partial x_{i}}=0
$$

and the momentum equation

$$
\frac{\partial \bar{u}_{i}}{\partial t}+\frac{\partial}{\partial x_{j}}\left(\bar{u}_{i} \bar{u}_{j}\right)=-\frac{1}{\rho} \frac{\partial \bar{p}}{\partial x_{i}}-\frac{\partial \tau_{i j}}{\partial x_{j}}+v \frac{\partial^{2} \bar{u}_{i}}{\partial x_{i} \partial x_{j}} .
$$

where $\bar{u}_{i}$ are the three Cartesian velocity components of the resolved large scales, $\bar{p}$ is the corresponding pressure and $R e$ is the Reynold number. During the filtering process, an additional term appears in the momentum equation, i.e., the subgrid scale (SGS) stress term, $\tau_{i j}$, which should be modeled and describes the influence of the non-resolved smallscale structures on the large eddies. The SGS stress term is given by

$$
\tau_{i j}=\overline{u_{i} u_{j}}-\bar{u}_{i} \bar{u}_{j}
$$

The SGS used here was proposed by Smagorinsky (1963) and Lilly (1966). The SGS stresses are related to the strain rate tensor by a SGS viscosity, $v_{T}$ :

$$
\tau_{i j}-\frac{1}{3} \delta_{i j} \tau_{k k}=2 v_{T} \bar{S}_{i j}
$$

where $\delta_{i j}$ is the Kronecker delta. The SGS viscosity is given by:

$$
v_{T}=\rho\left(C_{s} D_{\text {wall }} \Delta\right)^{2}|\bar{S}|
$$


where $C_{s}$ is the Smagorinsky constant $\left(C_{s}=0.1\right.$ in the present simulation), $D_{\text {wall }}$ the Van Driest wall damping factor, $\Delta$ the filter width and $|\bar{S}|$ the magnitude of the large-scale strain-rate tensor

$$
\bar{S}_{i j}=\frac{1}{2}\left(\frac{\partial \bar{u}_{i}}{\partial x_{i}}+\frac{\partial \bar{u}_{j}}{\partial x_{i}}\right)
$$

This combination (Smagorinsky / Van Driest wall damping) proved to be effective in the simulation of particle deposition on the internal walls of a vertical pipe flow. More complex SGS models have appeared in the literature (for example, the dynamic SGS eddy viscosity models), but they are beyond the scope of the present work. 


\section{Glossary}

\section{Asbestosis}

A chronic inflammatory and fibrotic medical condition affecting the parenchymal tissue of the lungs caused by the inhalation and retention of asbestos fibers.

\section{Choana}

The posterior nasal aperture, the opening into the nasopharynx.

\section{Coronal}

Of, relating to, or having the direction of the coronal suture or of the plane dividing the body into front and back portions.

\section{Gastrointestinal}

Of or relating to the stomach and intestines.

\section{Hygroscopicity}

The ability to attract water molecules from the surrounding environment through either absorption or adsorption.

\section{Krypton}

A chemical element with the symbol $\mathrm{Kr}$ and atomic number 36 .

\section{Lysozyme}

An enzyme occurring naturally in egg white, human tears, saliva, and other body fluids, capable of destroying the cell walls of certain bacteria and thereby acting as a mild antiseptic.

\section{Monodisperse}

A collection of objects are called monodisperse if they have the same molecular mass. 


\section{Nasopharynx}

The uppermost part of the pharynx, that lies behind the nose and above the level of the soft palate: it differs from the oral and laryngeal parts of the pharynx in that its cavity always remains patent (open).

\section{Olfactory}

Of or relating to the sense of smell.

\section{Olfactory region}

The area on and above the superior turbinates and on the adjoining nasal septum where the mucous membrane has olfactory epithelium and olfactory glands.

\section{Olfactory bulb}

One of two enlargements at the terminus of the olfactory nerve at the base of the brain just above the nasal cavities.

\section{Otolaryngology}

The branch of medicine that deals with diagnosis and treatment of diseases of the ear, nose, and throat.

\section{Post-mortem}

Of or relating to a medical examination of a dead body.

\section{Rhinometry}

A technique for measuring the nose or its cavities.

\section{Silicosis}

A form of occupational lung disease caused by inhalation of crystalline silica dust, and is marked by inflammation and scarring in forms of nodular lesions in the upper lobes of the lungs.

\section{Sinus}


Various air-filled cavities in the bones of the skull, especially one communicating with the nostrils.

\section{Stereolithography}

A three-dimensional printing process that makes a solid object from a computer image by using a computer-controlled laser to draw the shape of the object onto the surface of liquid plastic.

\section{Stochastic}

Random. Non-deterministic behaviour.

\section{Therapeutic}

Tending to cure or restore to health.

\section{Threshold}

The point that must be exceeded to begin producing a given effect or result or to elicit a response.

\section{Turbinate}

The scroll-shaped turbinate bones in the nasal passages (also referred to as conchae). 